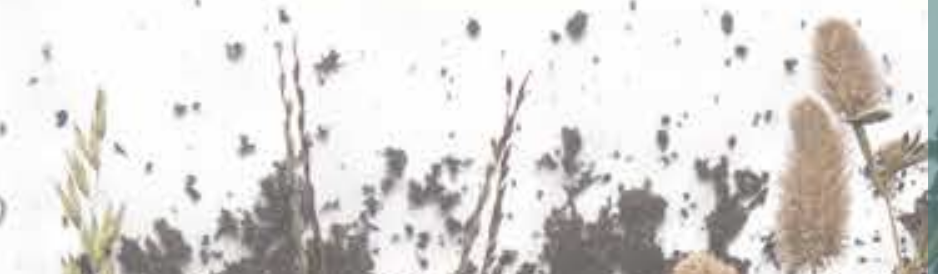
NATURAL CQMMUNITY DYNAMICS PLANT-SOIL INTERACTIONS IN

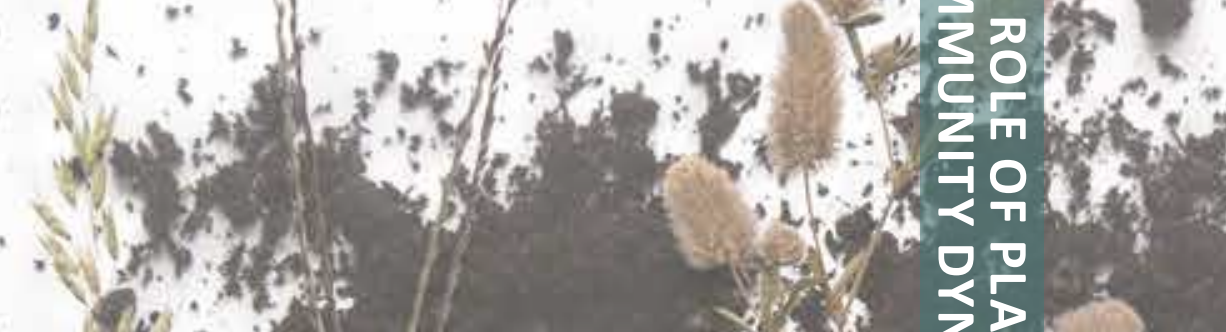
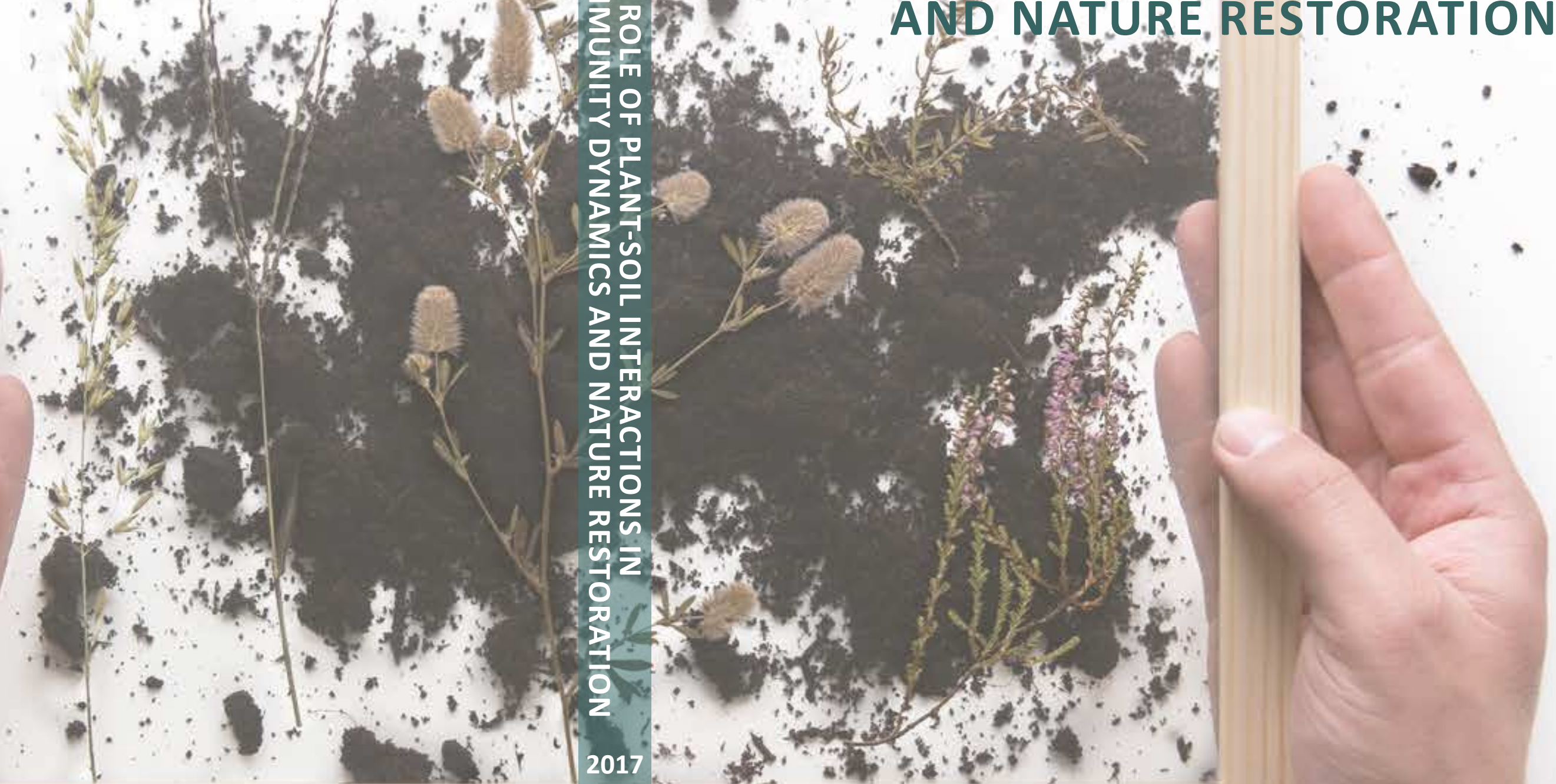

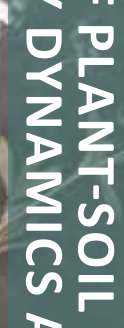

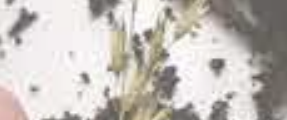

D.

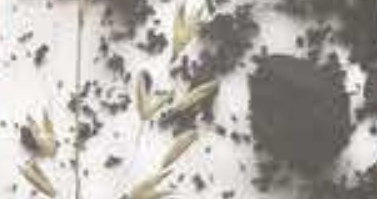

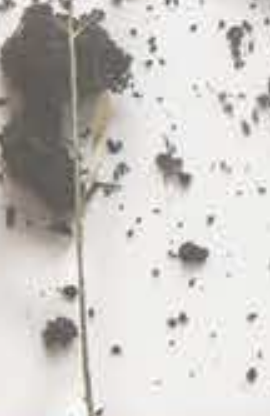

$4 a^{2}$
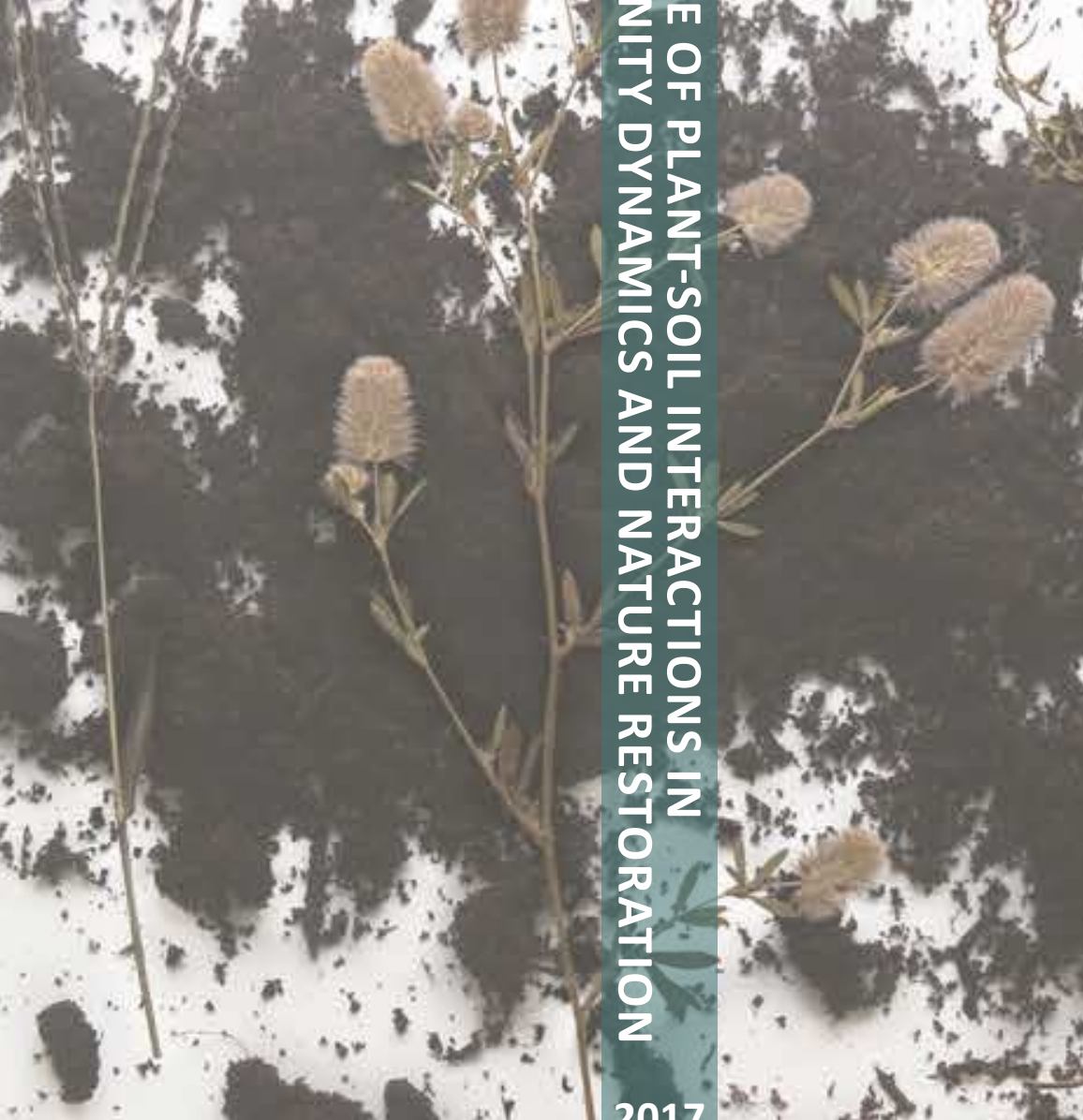

DNATURE, RESTORATION

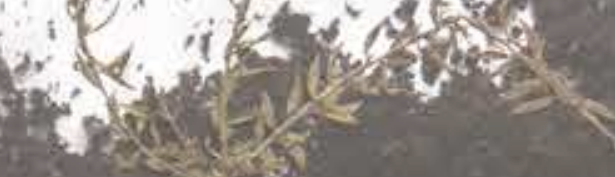



The steering role of plant-soil interactions in natural community dynamics and nature restoration

\author{
E. R. Jasper Wubs
}




\section{Thesis committee}

\section{Promotors}

Prof. Dr W.H. van der Putten

Special Professor Functional Biodiversity

Wageningen University \& Research and Netherlands Institute of Ecology (NIOO-KNAW)

Prof. Dr T.M. Bezemer

Special Professor Ecology of Plant-Microbe-Insect Interactions

Leiden University and Netherlands Institute of Ecology (NIOO-KNAW)

\section{Other members}

Prof. Dr H. de Kroon, Radboud University Nijmegen

Prof. Dr R.H. Marrs, University of Liverpool, United Kingdom

Prof. Dr L. Mommer, Wageningen University \& Research

Prof. Dr J.H.J. Schaminée, Wageningen University \& Research and Radboud University Nijmegen

This research was conducted under the auspices of the C.T. de Wit Graduate School for Production Ecology \& Resource Conservation (PE\&RC). 


\title{
The steering role of plant-soil interactions in natural community dynamics and nature restoration
}

\author{
Engel Reinder Jasper Wubs
}

Thesis

submitted in fulfilment of the requirements for the degree of doctor at Wageningen University by the authority of the Rector Magnificus Prof. Dr A.P.J. Mol, in the presence of the Thesis Committee appointed by the Academic Board to be defended in public on Wednesday 31 May 2017 at 4 p.m. in the Aula. 
E. R. Jasper Wubs

The steering role of plant-soil interactions in natural community dynamics and nature restoration,

242 pages.

PhD thesis, Wageningen University, Wageningen, the Netherlands (2017)

With references, with summaries in Dutch and English

ISBN: 78-94-6343-444-7

DOI: http://dx.doi.org/10.18174/412967 
Voor mijn ouders 



\section{Contents}

1 General introduction 9

$2 \quad$ Soil inoculation steers restoration of terrestrial ecosystems

$3 \quad$ Potential for synergy in soil inoculation for nature restoration by mixing inocula $\quad 57$ from different successional stages

$4 \quad$ Steering community development - how do sowing and soil inoculation affect $\quad 75$ above- and belowground communities over two decades?

$5 \quad$ Effects of spatial plant-soil feedback heterogeneity on plant performance in 99 monocultures

$6 \quad$ Spatial plant-soil feedback heterogeneity promotes plant diversity through $\quad 135$ equalizing competitive ability

$7 \quad$ Temporal carry-over effects in sequential plant-soil feedbacks $\quad 157$

$8 \quad$ General discussion \& synthesis $\quad 177$

$\begin{array}{ll}\text { References } & 193\end{array}$

$\begin{array}{ll}\text { Summary / samenvatting } & 221\end{array}$

$\begin{array}{ll}\text { Acknowledgements / dankwoord } & 231\end{array}$

About the author $\quad 235$

$\begin{array}{ll}\text { List of publications } & 237\end{array}$

PE\&RC Training and Education Statement $\quad 239$ 
CHAPTER 1

General introduction 
Biodiversity is a fundamental characteristic of ecosystems and it is of great concern that biodiversity is declining (Vitousek et al. 1997, Wilson 2011, Cardinale et al. 2012, Veresoglou et al. 2015). Therefore, the ultimate goal of many nature conservation organizations is to conserve and restore natural areas and particularly their constituent species: their biodiversity. However, both the re-creation and management of biodiverse ecosystems are likely to be more successful when they are based on a fundamental understanding of the regulation of biological communities (Walker et al. 2007). In reverse, lessons learned during ecological restoration can highlight gaps in our fundamental understanding of biological communities and ecosystems. As such, reconstruction of natural ecosystems provides the "acid test" of our ecological understanding (Bradshaw 1983, Ehrenfeld and Toth 1997, Walker et al. 2007).

In terrestrial ecosystems plants interact with a diverse set of organisms. Next to e.g. above-ground herbivores these include many important players that live in the soil and the interactions with soil organisms are thought to importantly affect the composition and functioning of plant communities (Wardle et al. 2004, Van der Heijden et al. 2008, Bardgett and van der Putten 2014). After a natural or human-mediated disturbance (Grime 2001), or when new terrestrial habitat becomes available, both plant and soil communities starts to develop anew. The sequence of changes in community composition that occurs in the subsequent years and decades is called succession (Grime 2001, Walker and Wardle 2014) and understanding this process may help in the successful restoration of degraded ecosystems. The interplay between plants and their associated soil communities has been a key focus of research in ecology in recent years, particularly with respect to their role in succession, plant community composition and diversity. However, the novel insights have not yet been widely applied in restoration ecology. In this thesis I aim to understand 1) how knowledge of plant-soil interactions may help to improve restoration of natural ecosystems, and 2) how these interactions affect plant diversity and composition.

\section{Plants and soil biota}

The soil community consist of myriad organisms whose body width spans five orders of magnitude, from a micrometre to $10 \mathrm{~cm}$ (Giller 1996, Coleman et al. 2004). Moreover there is also great taxonomic diversity belowground: one gram of soil can contain more than 5000 species (Torsvik et al. 2002, Bardgett and Wardle 2010, Bardgett and van der Putten 2014). Many groups of soil biota are involved in the complex detrital food web that decomposes and mineralizes organic matter, 
but there are many more interactions belowground that affect terrestrial plant communities including root herbivores and plant mutualists (Van der Heijden et al. 2008, Bardgett and Wardle 2010). Plants determine the amount and quality of resources available for the organisms belowground (Swift et al. 1979, Bardgett and Wardle 2010). In turn, soil organisms have profound effects on productivity, biodiversity and composition of terrestrial plant communities (Wardle et al. 2004, Bardgett and Wardle 2010, De Kroon et al. 2012).

While plants provide the litter and root exudates that fuel the soil detrital food web (Bardgett and Wardle 2010), the activity of the decomposer community strongly determines the rates of nutrient mineralization and thus indirectly affects plant productivity (Swift et al. 1979, Wardle et al. 2004, Bardgett and Wardle 2010). The detrital soil food web has been described as consisting of two alternative energy channels where certain nematodes and mites feed on fungi, while others mainly consume bacteria (Bardgett et al. 1993, De Ruiter et al. 1994, Moore et al. 2003). The bacterial channel is dominant in soils with more labile organic matter (e.g. low $\mathrm{C}: \mathrm{N}$ ratios), which allows fast nutrient cycling, while the fungal channel plays a more important role in soils with recalcitrant organic matter, usually on more acidic soils (Bardgett and Wardle 2010). Although microbes are responsible for the biochemical decomposition of detritus, soil fauna (e.g. many arthropods, earthworms) play an important role in 1) the mechanical disruption of dead plant material, thereby exposing the organic material for microbial attack, and 2) mixing the microbes with the detritus (Bradford et al. 2002, Coleman et al. 2004, Bardgett and Wardle 2010).

Whereas decomposers interact with plants indirectly, through mineralization of dead organic material, root associated organisms, such as root herbivores, pathogens and symbiotic mutualists, affect plant performance directly. Root herbivores include various plant-parasitic nematodes (Bongers and Bongers 1998) and larval stages of certain insects (e.g. Coleoptera, some Diptera and some Lepidoptera, amongst others; Brown and Gange 1989, 1990). Mutualists include several kinds of mycorrhizal fungi (Van der Heijden et al. 2008), $\mathrm{N}_{2}$-fixing diazotrophic bacteria (e.g. Rhizobia; Santi et al. 2013), fungal endophytes (Clay 1990), and plant growth-promoting rhizobacteria (Lugtenberg and Kamilova 2009). Among others, viruses, bacteria and nematodes can all be plant pathogens (Agrios 1978), but the best studied group are the fungi (e.g. Phytophthora, Fusarium; Jarosz and Davelos 1995). The fungal phylogeny shows that the plant pathogenic lifestyle has evolved on numerous occasions from saprotrophic fungi (James et al. 2006). Similarly, 
mutualistic relationships are not stable and may change - even to antagonism over evolutionary and to a lesser extent over ecological timescales (Kiers et al. 2010, Johnson 2010). Finally, predators of soil organisms, e.g. predaceous collembola, mites or nematoda, can also affect plants indirectly through their impact on the populations of herbivores or plant-associated microbes (Moore et al. 2003, Bardgett and Wardle 2010). For example, entomopathogenic nematodes can infect and kill root-feeding caterpillars of the ghost moth (Strong et al. 1996). So, not only is the soil rich in its taxonomic diversity, there is also a wide array of functional diversity belowground that can affect plants both directly and indirectly.

\section{Plant-soil feedback}

The interactions between soil organisms and plants have traditionally been studied by manipulating specific pairs of plants and soil organisms (Bever 1994). However, plant species can also respond to soil organisms whose identity is unknown. Moreover, given the many interactions among soil biota, the soil community needs to be studied as a whole in order to fully capture its effects on plants. Plant-soil feedback (PSF) studies overcome this problem by, in the first instance, considering the soil community as a 'black box' and directly studying the net response of a plant to a soil community in terms of growth and ontogeny (Bever 2003, Brinkman et al. 2010). The basic idea is that plants, e.g. through root exudates and other chemical cues, attract and promote certain soil biota while repressing others (Bever et al. 1997, Kulmatiski et al. 2008, Van der Putten et al. 2013). Subsequently, the changed soil community can affect the growth and performance of the plant (feedback). This can be studied empirically by first having a certain plant grow in a soil (the conditioning phase) and then growing the same and/or a different plant species in the conditioned soil (test phase) and measuring its response, e.g. biomass production. The conditioning phase can take place either in the greenhouse or in the field. In the latter case soil is collected from stands of the focal species or community (Kulmatiski and Kardol 2008).

The experimental design can include sterilized vs. non-sterilized soil or 'own' vs. 'foreign' soil treatments to either study the net effect of the presence or absence of the soil community as a whole or net effects of conditioning of the soil community by specific plants (Kulmatiski and Kardol 2008, Brinkman et al. 2010). Plant-soil feedbacks are considered positive when they improve the performance of plants and negative when performance is reduced. The PSF effect of a plant species on conspecifics is termed direct, intraspecific or conspecific feedback, while PSF effects on other species are termed indirect, interspecific or heterospecific feedback 
(Van der Putten et al. 2013). Naturally, as plants may simultaneously alter many soil biotic and abiotic properties (Ehrenfeld et al. 2005, Mazzoleni et al. 2015), pinpointing the exact causes of the feedback may be difficult (Brinkman et al. 2010). Nevertheless, in recent years PSF studies have led to important advances in understanding of plant communities and their dynamics, such as invasion and range expansion, succession, and plant community composition and diversity (Van der Putten et al. 2013). However, relatively little is known about how knowledge about plant-soil feedbacks may aid nature restoration.

\section{Plant-soil interactions for nature restoration}

During restoration both plant and soil communities will undergo successional changes and it is thought that restoration efforts will be much more successful if the processes leading to successional dynamics are understood (Young et al. 2001, Suding et al. 2004, Walker et al. 2007, Cramer et al. 2008). In addition, previous human activities can create biotic and abiotic legacies that prevent unassisted transition to restored ecosystems and targeted interventions to alleviate these constraints are required (Suding et al. 2004, Cramer et al. 2008). It has been suggested that in terrestrial ecosystems plant-soil interactions play a significant role in succession (Van der Putten et al. 1993, Kardol et al. 2006, Bauer et al. 2015, Castle et al. 2016) and may be key to successful ecological restoration (Suding et al. 2004, Young et al. 2005, Eviner and Hawkes 2008, Harris 2009, Kardol and Wardle 2010).

\section{Natural succession - above- and belowground}

Natural succession is the change in species composition over time (Horn 1974, Walker et al. 2007), brought about by endogenous ecological processes, such as colonization, competition and predation (Grime 2001, Walker and Wardle 2014). Primary succession describes those community developments that occur after new habitat has been created, e.g. formation of new rocks by volcanoes (Walker and del Moral 2003), land appearing from below retreating glaciers (Chapin et al. 1994, Castle et al. 2016), and on primary dunes (Van der Putten et al. 1993). Secondary succession, on the other hand, describes the changes in community composition that occur in existing habitats after a disturbance, such as after a major fire outbreak (Wardle et al. 1997) or after abandonment of former agricultural land (Pickett 1982, Cramer et al. 2008). The predictability of successional changes in community composition have been debated from the inception of the concept (c.f. Clements 1916, Gleason 1917, Egler 1954). The current view suggests that the exact species composition may be unpredictable, and may depend strongly on initial species composition and dispersal, but that communities converge to 
similar functional groups of taxa (Wilson and Roxburgh 1994, Fargione et al. 2003, Fukami et al. 2005, Cramer et al. 2008).

In the context of nature restoration and biodiversity conservation the focus has been on the understanding of patterns and processes in secondary succession (Walker et al. 2007, Cramer et al. 2008). In this thesis, I will focus on the successional changes occurring after abandonment of arable fields, also known as old-field succession (Pickett 1982, Cramer et al. 2008). Around the world many arable fields, in fact around 200 million hectares, have been abandoned during the past decades, because production was insufficient to be economically feasible (Cramer et al. 2008). These fields now provide important opportunities for the conservation of biodiversity (Walker et al. 2004, Cramer et al. 2008). In particular, I will use former agricultural fields on Pleistocene sandy soils in the south Veluwe region of the Netherlands as study system.

Historically the south Veluwe area has been used mainly for small-scale arable farming, where soil fertility was supplemented by applying sods cut from the surrounding landscape (Breman et al. 2009). In addition, large areas of noncultivated land were used for sheep herding. From the $13^{\text {th }}$ century onwards this created a mosaic of open land, mainly grasslands and heathlands, as well as woodlands. Subsequently, in the $18^{\text {th }}$ and $19^{\text {th }}$ centuries, large parts of the area were planted with trees (e.g. Pinus, Quercus) to provide alternative sources of income and to combat ongoing soil degradation leading to drift sands (Breman et al. 2009). In the $20^{\text {th }}$ century large arable farms were established, cultivating crops such as maize and wheat. Finally, from 1985 onwards many of these fields were abandoned by farmers and sold to the State, which handed these fields over to nature conservation organizations. Although globally economic factors are the main reason for the abandonment of arable fields (Cramer et al. 2008), in this case the government actively pursued these lands as part of a national scheme targeted at increasing habitat connectivity across Dutch landscapes. Besides the extensive woodlands that occur in the area, many of these former fields are being ecologically restored to species-rich, nutrient-poor grasslands and dry heathlands. These open systems are typically managed by low-intensive grazing of natural and introduced vertebrate herbivores, such as roe deer, fallow deer, red deer, horses and Scottish Highland cattle. In addition, wild boar are an important part of the vertebrate fauna that regularly create soil disturbances in much of the area (Bruinderink and Hazebroek 1996). The south Veluwe has been the focus of research on succession and restoration for a long time and there is important information available on 
above- and belowground community processes (e.g. Van der Putten et al. 2000, Kardol et al. 2005, Van der Wal et al. 2006, Holtkamp et al. 2011, Van de Voorde et al. 2012, Morriën et al. 2017).

In general, the agricultural practises associated with arable farming, including tillage, fertilization and the use of pesticides, cause important and continued disturbance to plant and soil communities (Cramer et al. 2008). As a result, intensive agricultural use substantially reduces the diversity of the soil community and the functioning of the soil food web (Bardgett et al. 1993, De Vries et al. 2013, Tsiafouli et al. 2015). After abandonment many ruderal plant species are recruited from the seedbank (Bazzaz 1968, Bekker et al. 1997). This cohort of mostly annual plants is rapidly replaced by more competitive biennial and perennial species of grasses and forbs (Egler 1954, Cramer et al. 2008). Subsequently, these communities are replaced by shrubs and later usually by trees (Egler 1954, Grime 2001, Cramer et al. 2008, Prach et al. 2014). The seeds and vegetative fragments of these later groups of plants may already be present in the soil (Egler 1954, Grime 2001) or disperse from the surrounding area (Zobel 1997, Bakker and Berendse 1999). The transition to forest may be delayed or halted by vertebrate grazers (Davidson 1993, Bullock and Pakeman 1997, Olff et al. 1999), rooting by e.g. wild boar (Bruinderink and Hazebroek 1996), and traditional agricultural practises such as sod cutting and haymaking, as was common on marginal soils for much of recent human history (Bignal and McCracken 1996, Bakker et al. 2002, Breman et al. 2009). In these cases, grasslands or dry heathlands persist. When fertility is continually reduced by mowing, or by grazing when herds are moved off-site during the night, succession will proceed to herbaceous vegetation typical of oligotrophic conditions (Berendse et al. 1992, Marrs 1993, Bakker et al. 2002). High soil fertility, e.g. due to past fertilizer application and nitrogen deposition from the air (Bobbink et al. 1998, Duprè et al. 2010), favours competitive and fast growing early-succession species (Grime 2001, Cramer et al. 2008, Duprè et al. 2010). Once established these competitive early-successional plant communities may prevent the establishment of the later successional grassland communities for prolonged periods of time (Cramer et al. 2008, Bartha et al. 2014).

During old-field succession the plant community changes to species with low litter quality (Baer et al. 2002, Bardgett and Wardle 2010). In response the soil decomposer food web is thought to shift from a copiotrophic bacteria-dominated system to a oligotrophic fungal-dominated system (Bardgett et al. 1993, De Vries et al. 2007, Bardgett and Wardle 2010). However, on ex-arable land fungal biomass 
does not increase after the first years of land abandonment (Van der Wal et al. 2006). Instead, fungal activity increases with time since abandonment, showing that fungi indeed pass on more energy to higher trophic levels (Morriën et al. 2017). The bacterial community has also been shown to shift in species composition. For instance, members of the bacterial phyla Verrucomicrobia and Acidobacteria are thought to be a key component of the microbial community in oligotrophic sites (Ramirez et al. 2012, Fierer et al. 2013). However, this is a new and actively developing research field, and the association of microbial taxa to habitats is by no means complete (e.g. Suleiman et al. 2013). In addition to the saprophytic fungi, also species of arbuscular mycorrhizal fungi in grasslands and ericoid mycorrhiza in heathlands become increasingly abundant as succession proceeds (Johnson et al. 1991, Barni and Siniscalco 2000, Morriën et al. 2017), and their species composition also changes in later stages of succession (Johnson et al. 1991, Barni and Siniscalco 2000). These changes in the basal part of the food web are further reflected by the higher trophic levels, such as nematodes, enchytraeids, collembola and mites. For instance, there are shifts from plant-feeding and bacterial-feeding nematodes to fungal-feeding and predaceous nematodes (Korthals et al. 2001, Verschoor et al. 2001, Kardol et al. 2005, Hánél 2010). Therefore, the soil foodweb assembles from a more opportunistic, loosely interacting, copiotrophic community, to one where interactions are tight and nutrient cycling is highly efficient (Neutel et al. 2007, Holtkamp et al. 2011, Morriën et al. 2017).

The changes in soil community composition contribute importantly to plant succession (Van der Putten et al. 2013). In the early phase of old-field succession plants tend to experience negative soil feedback (Kardol et al. 2006, Bauer et al. 2015), mainly because these earlier colonizers are poorly defended (Van der Putten 2003) and form few associations with soil-borne mutualists (Reynolds et al. 2003, Hoeksema et al. 2010). During succession the current plant species are replaced by later successional species that are not susceptible to the pathogens of early successional species (Kardol et al. 2006, Bauer et al. 2015). In addition, these later-successional plant species benefit more from soil-borne mutualists (Janos 1980, Bever et al. 2012), which can improve plant defence and access to soil resources (Smith and Read 1997). As a consequence the PSF experienced by the plants becomes increasingly positive during old-field succession (Kardol et al. 2006, Bauer et al. 2015).

Natural plant and soil communities can change importantly over decadal timescales (Walker and Wardle 2014). Furthermore, the communities are strongly 
interdependent as plants provide the primary resources, in the form of root exudates or plant litter, for the soil food web, while the soil community affects plant performance through antagonistic and mutualistic interactions. The availability of plant propagules determines which plant species can potentially colonize the site, while the abiotic environment, particularly climate, hydrology, soil type and site fertility, acts as a filter and determines which species actually establish (Zobel 1997, Ter Steege and Zagt 2002, Prach et al. 2014). However, research over the past two decades has highlighted that soil community restoration may be a key aspect of successful nature restoration (Reynolds et al. 2003, Eviner and Hawkes 2008, Harris 2009, Kardol and Wardle 2010).

\section{Nature restoration in old-fields}

Initially old-fields were simply left to recover on their own through unassisted successional developments (Bakker and Berendse 1999). This method can be successful provided that the appropriate abiotic conditions, including low-nutrient inputs, are in place, and the desired species can colonize from the surrounding landscape (Bonet 2004, Albert et al. 2014, Prach et al. 2014). However, unassisted restoration may take a number of decades or longer (Cramer et al. 2008, Redhead et al. 2014) and in many cases such natural recovery projects have failed to reach their targets (Muller et al. 1998, Lockwood and Pimm 1999, Bakker and Berendse 1999, Walker et al. 2004). Globally, unassisted recovery may become less likely in the future as old-fields that become available for restoration are increasingly located in intensively managed landscapes with few remnant patches of target habitat (Vellend 2003, Cramer et al. 2008). In addition, land-use history, such as tillage and fertilization during arable cultivation, can leave historical legacies in the soil (Kulmatiski et al. 2006, McLauchlan 2006, Kulmatiski and Beard 2011), which can strongly affect subsequent old-field succession (Bonet 2004, Cramer et al. 2008). Overcoming these legacies requires active intervention. The challenge is to find the right interventions that will steer successional community developments into the direction of the desired target communities (Prach et al. 2007).

The fertility of the soil and the presence of appropriate plant propagules have long been identified as important constraints for successful restoration of species rich grasslands and dry heathlands (Bakker and Berendse 1999, Walker et al. 2004). Soil fertility can be reduced through various means, such as mowing, grazing (Marrs 1993, Bakker et al. 2002), and phosphorus-mining by specialized plant species (Schelfhout et al. 2015). In it most extreme form soil fertility reduction is implemented by topsoil removal, in which the whole organic top-layer is removed 
(Verhagen et al. 2001, Walker et al. 2004, Pywell et al. 2007, Jaunatre et al. 2014). This layer can be more than $20-100 \mathrm{~cm}$ in depth and this is where most of the nutrients are concentrated (Van der Wal et al. 2007, Bekker 2009). Although highly effective in reducing soil fertility to oligotrophic target conditions, topsoil removal often does not lead to successful restoration by itself (Verhagen et al. 2001, Kardol et al. 2008, Bekker 2009). Along with the top soil also the propagules of plants and soil biota are removed, which may importantly hinder plant community development (Verhagen et al. 2001, Kardol et al. 2008). Methods to introduce plant propagules include adding cut hay from donor fields, direct sowing of seeds, or even directly planting species (Van der Putten et al. 2000, Donath et al. 2007, Kardol et al. 2009, Wallin et al. 2009, Kiehl et al. 2010). However, the role of plant-soil interactions, and consequently soil community developments, in succession and restoration have only recently begun to attract attention.

Recent studies have highlighted the key role that soil community restoration may play in the successful restoration of ecological communities (Reynolds et al. 2003, Eviner and Hawkes 2008, Harris 2009, Kardol and Wardle 2010). On the one hand efforts have focussed on altering nutrient cycling in the soil food web by supplying recalcitrant forms of organic matter, which is thought to cause nitrogen immobilization and thus indirectly reduce site productivity and improve conditions for oligotrophic communities (Blumenthal et al. 2003, Averett et al. 2004). Other workers have focussed on the direct interactions between plants and beneficial soil organisms. Soil inoculation has been applied for at least a hundred years and early work focussed on re-establishment of crop-rhizobia interactions to increase the yield of legume crops (Brown 1918). Many workers identified beneficial soil organisms, particularly mycorrhizal fungi (e.g. Middleton and Bever 2012, Middleton et al. 2015), and used these in specific soil inocula to improve target plant establishment and growth. In other studies soil material was integrally transferred without first selecting particular beneficial organisms (Vécrin and Muller 2003, Kardol et al. 2009, Box et al. 2011, Bulot et al. 2014). While taxon-focussed inoculations have received much attention, it has been shown that inoculation with whole soil communities is more effective (Rowe et al. 2007, Hoeksema et al. 2010, Paluch et al. 2013, Emam 2016). This type of whole-community transfer appears in different forms (Bullock 1998). When whole soil monoliths are transported it is often called turf or soil transplantation. When the soil is homogenised prior to application it is called soil transfer and when only small amounts of soil are applied per unit area it is called soil inoculation. Recently, soil transfer and soil inoculation have been applied to reintroduce appropriate plant propagules (Bullock 1998, Kiehl et al. 
2010), but also to co-introduce soil communities (Kardol et al. 2009, Carbajo et al. 2011, Brinkman et al. 2012, Hamman and Hawkes 2013). Besides differences in method, the area treated with donor soil also varies strongly in size $\left(625 \mathrm{~cm}^{2}\right.$ to $0.4 \mathrm{ha}$ ). Restoration of target plant communities was especially successful in transplantations at large spatial scales (Pywell et al. 1995; Bruelheide and Flintrop 2000; Vécrin and Muller 2003). However, the success was not restricted to whole turf transplantations: soil transfer methods were similarly successful (Good et al. 1999; Vécrin and Muller 2003). The smaller-scale transplantations were generally less successful (Pärtel et al. 1998, Kardol et al. 2009, Middleton and Bever 2012).

While there have been several studies exploring the potential of plant-soil interactions to promote nature restoration, including soil translocation methods, several key questions have not yet been addressed. Surveys have clearly demonstrated that different plant communities, along succession and also across spatial gradients, are associated with different soil communities. However, interventions in these interactions have often been limited to testing whether there was an effect, whereas it is still unclear to what extend differences in the composition of the soil inoculum can lead to different trajectories of ecosystem development. If this is the case, soil inoculation could provide nature managers with much more control over the successional developments in their fields. In addition, while the feedbacks between plants and soil communities are increasingly studied, it is unclear whether aboveor belowground interventions exert the dominant influence over the development of the ecosystems in the longer term. Finally, although field experiments existed before, appropriately replicated experiments at a spatial scale that is relevant for the practice of ecological restoration have so far been lacking. Addressing these questions under the same environmental conditions will allow us to better identify which interventions in plant-soil interactions are most appropriate for successful restoration of desired semi-natural ecosystems.

\section{Maintenance of plant diversity}

Knowledge on how to restore ecosystems is increasing, but the success in practise varies. This is in part because of the goals that are set as targets for restoration (Perring et al. 2015). For instance, restoring plant cover and a number of common ('matrix') plant species is relatively straightforward (Baer et al. 2002), but creating conditions that allow for coexistence of both locally dominant and rare subordinate species proves much more elusive (Walker et al. 2004, Bekker 2009). Fundamental knowledge on how plant diversity is regulated is needed to achieve these more advanced goals. 
The distribution of plant species is simultaneously affected by factors that operate at different spatial (and temporal) scales, like variations in climate, geology, soil types, herbivores, and pathogens (Tilman 1982, Grime 2001, Bardgett and Wardle 2010). Plants respond to the heterogeneous spatial mosaic created by these drivers and together with processes like dispersal (Ter Steege and Zagt 2002, Svenning et al. 2008) this determines the vegetation's composition, spatial arrangement and diversity. Many aspects of the distribution of plants and the temporal changes therein are the subject of active investigation, however, one aspect that has received a large amount of attention, but remains unresolved is what maintains plant diversity on relatively small spatial scales (Chesson 2000, Wilson 2011, De Kroon et al. 2012, Bever et al. 2015). Relatively small means the scale where plants interact (Casper et al. 2003, Wilson 2011). Looking at the world records of species richness for grasslands we can find up to 42 species in an area as small as $20 \times 20 \mathrm{~cm}$ and up to 89 species in $1 \mathrm{~m}^{2}$ (Wilson et al. 2012). The question is what drives this large diversity on such small spatial scales (Hutchinson 1959, 1961, Wilson 2011)?

A long-standing view is that spatial heterogeneity in environmental conditions allows for the coexistence of many plant species (MacArthur 1972, Tilman 1982, 2011, Chesson 2000, Silvertown 2004, Letten et al. 2016). Both observational and experimental data have supported this assertion. For example, the number of plant species on islands in Great Britain was best predicted by the number of soil types on those islands (Johnson and Simberloff 1974) and in a manipulative experiment the introduction of small topographical heterogeneity in artificial wetlands substantially increased their species richness (Vivian-Smith 1997). However, recent meta-analyses have shown that the sign of the heterogeneity-diversity relationship depends on the spatial grain (i.e. patch size) of the heterogeneity (Lundholm 2009, Tamme et al. 2010). When the size of the manipulated or observed patches drops below about $20 \times 20 \mathrm{~cm}$, spatial heterogeneity does not promote, but rather reduces plant diversity.

As an explanation it has been suggested that plant species may be differentially adapted to exploit such small-scale heterogeneity (Hutchings et al. 2003, Tamme et al. 2010). Plants are known to differ in their root foraging abilities (Grime 2001, De Kroon and Mommer 2006), allowing some species to rapidly exploit smallscale belowground heterogeneity in resources (Hutchings et al. 2003, Baer et al. 2004, Wijesinghe et al. 2005, Eilts et al. 2011). Consequently, high root plasticity can speed up competitive exclusion directly, by rapidly foraging for nutrients. On the other hand, high root plasticity, can also lead to significant costs to plants, e.g. 
due to high root turnover, and be a disadvantage in the long term (Fransen and De Kroon 2001). Recently, an experimental study demonstrated that small-scale heterogeneity in nutrients reduces plant diversity because it indirectly enhances the competition for light through selection for high root foraging abilities (Gazol et al. 2013). This suggests that spatial heterogeneity in abiotic conditions will only promote diversity when the grain of the heterogeneity exceeds a plant's zone of influence (sensu Casper et al. 2003). Abiotic heterogeneity thus primarily promotes plant beta diversity and not alpha diversity (Whittaker 1960, Wilson 2011). Instead, it has been hypothesized that plant antagonists, particularly soil-borne pathogens, may be key drivers of plant diversity at small spatial scales (Bradley et al. 2008, Tamme et al. 2010, De Kroon et al. 2012, Bever et al. 2015, Bennett and Cahill 2016).

Plant diversity maintenance trough plant antagonists, known as the JanzenConnell, or pest pressure, hypothesis (Janzen 1970, Connell 1971, Wilson 2011), operates through negative conspecific density dependence. When a species becomes dominant in a community its enemies are more likely to find it and reduce its fitness, and vice versa when it is rare (Janzen 1970, Connell 1971). Negative conspecific density dependence has frequently been recorded in the field (Comita et al. 2014), in temperate forests (Packer and Clay 2000, Hille Ris Lambers et al. 2002, Johnson et al. 2012), tropical trees (Augspurger and Kelly 1984, Bell et al. 2006, Liu et al. 2012) and grasslands (Klironomos 2002, Petermann et al. 2008). In some cases the particular pest responsible has been identified. For instance, seedling recruitment and growth of the temperate tree Prunus serotina is known to be repressed under adult trees by the soil-borne oomycete genus Phytium (Packer and Clay 2000, 2003). Likewise, fungicide treatment targeting oomycetes removed the density dependent seedling mortality for the neotropical tree Sebastiana longicuspis (Bell et al. 2006). In most cases, however, the exact herbivore or pathogen responsible for the negative conspecific density dependence is not known (Wilson 2011, Bever et al. 2015).

More importantly, given the myriad interactions within the soil food web, it may not be one single taxon that is responsible for the negative density dependence. There have been several reports where multiple antagonists were involved (e.g. De Rooij-Van der Goes 1995, Morris et al. 2007, Bradley et al. 2008, Hersh et al. 2012), and it has been suggested that such co-infections may be the rule rather than the exception (Benítez et al. 2013, Raaijmakers and Mazzola 2016). Consequently, using strain inoculation-based methods may be inappropriate to 
study the impact of soil-borne antagonists on plant community composition and diversity. Therefore, a plant-soil feedback approach (see above), where the net effects of a whole soil community on plant community dynamics can be studied, is more suitable (Bever et al. 2015).

The results of PSF studies so far are in line with the idea that soil-borne enemies help maintain plant diversity at small spatial scales (Petermann et al. 2008, De Kroon et al. 2012, Van der Putten et al. 2013, Bever et al. 2015). In general, most direct PSF effects are negative, especially in grasses and forbs, (Klironomos 2002, Bever 2003, Petermann et al. 2008, Kulmatiski et al. 2008), showing that pest pressure builds as conspecifics condition the soil. The relative strength of intraspecific vs. interspecific feedbacks has been poorly investigated (Kulmatiski et al. 2008), although the available data suggests that conspecific PSFs tend to be more strongly negative than heterospecific feedback (Bever et al. 1997, Fitzsimons and Miller 2010, Van de Voorde et al. 2011). Mathematical models of PSF driven plant communities show that it can promote plant diversity at small spatial scales for many generations (Bever et al. 1997, Bever 2003, Bonanomi et al. 2005, Fukami and Nakajima 2013, Revilla et al. 2013). Together, this suggests that the net effect of interactions with the soil community may facilitate coexistence among competing plant species (Bever et al. 1997, 2015, Bever 2003, De Kroon et al. 2012). There are, however, two important gaps in knowledge with respect to PSFmediated plant species coexistence: 1) how do PSFs affect plant communities in realistic spatial settings and 2) what is the effect of PSF legacies, mediated though the presence of the resting stages of soil organisms, on plant community dynamics?

Theoretical models of spatial plant communities show that PSF can mediate coexistence if its effects are highly localized (Bonanomi et al. 2005, Fukami and Nakajima 2013, Mack and Bever 2014, Abbott et al. 2015). The spatial patterns of plant species turnover observed in these models are consistent with observed temporal dynamics of grassland plant communities recorded at small spatial scales (10 x 10 cm; Van der Maarel and Sykes 1993, Herben et al. 1993). However, thus far virtually all empirical PSF studies have been conducted in glasshouses and have been restricted to homogenized soils (Kulmatiski and Kardol 2008). In the field, however, soil biota are distributed heterogeneously in space (Ettema and Wardle 2002). In addition, studies that used field-conditioned soil or that were conducted in the field (De Rooij-Van der Goes et al. 1998, Casper and Castelli 2007) have so far been restricted to species that grow in relatively large monospecific clumps. However, many grassland species grow highly intermingled 
and a plant's belowground zone of influence, i.e. its lateral root spread, typically goes well beyond the extend of its aboveground canopy (Casper et al. 2003). Interestingly, grassland species that do not grow as extensive clones also cultivate their own particular soil community in the field, even when their roots are allowed to fully intermingle (Bezemer et al. 2010).

Several recent studies have explicitly manipulated the spatial distribution of PSFs. Here, plant performance was compared in treatments with soil patches that had been conditioned by different species (heterogeneous) to treatments where these soils were mixed homogeneously (Brandt et al. 2013, Burns and Brandt 2014, Hendriks et al. 2015a, b). These studies showed that spatial heterogeneity in PSFs can affect plant performance (Brandt et al. 2013) and can enhance the uptake of nutrients (Hendriks et al. 2015a). In addition, seedling establishment was higher in heterospecific conditioned patches than in conspecific patches (Burns and Brandt 2014), which is consistent with predictions of PSF-driven coexistence (Petermann et al. 2008, Bever et al. 2015). Finally, spatial heterogeneity of PSFs can alter root competitive interactions and, consequently, competitive hierarchies among plants (Hendriks et al. 2015b). However, these studies have so far been limited to interactions among pairs of plants, whereas there is no direct empirical evidence that heterogeneity of plant-soil feedbacks enhances plant diversity in communities (Hendriks et al. 2015b). In addition, a modelling study suggests that the spatial scale of the PSF (feedback neighbourhood size) will importantly affect plant community dynamics (Mack and Bever 2014). However, this has not yet been tested empirically.

The second gap in knowledge is how PSFs develop over time (Kardol et al. 2013). With some exceptions (Mazzola 1999, Klironomos 2002, Packer and Clay 2004), most empirical PSF studies have been restricted to a single cycle of conditioning and feedback. Models of PSF generated plant diversity implicitly assume that the feedback generated by a given species is the same across many generations (Bever et al. 1997, Bever 2003, Bonanomi et al. 2005, Fukami and Nakajima 2013). However, it is well known that many soil biota, including many plant pathogens, have resting stages that can remain in the soil for a long time (Agrios 1978, Miller et al. 1994, Felske and Akkermans 1998, Ettema et al. 2000, Coleman et al. 2004, Lennon and Jones 2011). Although it is often unclear precisely how long these resting stages stay viable, it can be expected that these accumulated propagules may germinate when conditions become favourable years later, e.g. when the roots the host plant re-enter the soil patch. As a result, biotic soil feedbacks may 
potentially carry-over across periods of soil conditioning by different species. In addition, dormant propagules are subjected to predation, e.g. by protozoa, which may reshape their community composition (Lennon and Jones 2011). It is unknown how repeated soil conditioning will affect plant performance, but it may be key to incorporate into our understanding of plant and soil interactions, and their consequences for plant diversity.

\section{Aims of this thesis}

In this thesis, I explore the role of plant-soil interactions in restoration of seminatural ecosystems on sandy soils in the Netherlands and their natural dynamics in space and time.

First, I will test whether inoculation of soil from later-successional ecosystems can enhance restoration in a real outdoor ecosystem, at a spatial scale that is relevant for ecological restoration projects. I will analyse a field experiment where soil from either dry heathlands or infertile grassland was inoculated into a recently abandoned ex-arable field and record the plant and soil community composition (Chapter 2). In this setup, I will test whether the two inocula can steer the development of the communities in the direction of their respective donor communities and whether the soil community is a driver of this process.

Having established the potential of belowground interventions to affect plant-soil interactions, I will subsequently test whether mixtures of soil inocula can lead to synergistic, antagonistic or strictly additive effects for nature restoration (Chapter 3). Specifically, in a greenhouse experiment, I test the hypothesis that a mixture of soil inocula from early- and late-successional ecosystems will enhance the growth of late-successional target plant species, while simultaneously repressing the growth of early-successional ruderal species.

Then, I investigate whether plant and soil community developments can mainly be steered by separate interventions or their interplay (Chapter 4). I test the hypothesis that simultaneous introduction of both mid-successional plants and mid-successional soil communities into an early-successional ex-arable field will speed up natural community development. Using data from a well-replicated long-term field experiment I describe the dynamics of the taxonomic composition of both the plant and nematode communities during 20 years following initial manipulation of plant and soil community composition. 
In chapters 2-4, I apply fundamental knowledge on plant-soil interactions to the practise of nature restoration by analysing results from field and greenhouse experiments. In chapters 5-7, I explore the spatio-temporal dimensions of plantsoil feedback in more detail by greenhouse studies. I examine how plant-induced changes in the soil feedback affect plant performance at the scale of individual plant interactions.

I will manipulate the spatial heterogeneity of plant-soil feedbacks to test the hypothesis that pest pressure from plant induced changes in soil community composition can enhance plant diversity at the spatial scale where individual plants interact. I use six mid-succession species that commonly occur in the exarable fields on sandy soils in the Veluwe region as a model system and grow them on soils with patchy or spatially homogeneous PSF. I examine the responses of these six species to PSF heterogeneity in monoculture (Chapter 5) and as part of four species communities (Chapter 6).

In the final experiment in this thesis I will investigate the temporal dynamics of plant-soil interactions in order to determine whether and how soil-conditioning of plants can carry-over from one generation to the next (Chapter 7). I condition soil in two consecutive phases using the same six mid-succession grassland species in a full factorial design. Subsequently, I record the performance of one of these six species in the third phase on each of the 36 conditioned soils. For this I use Jacobea vulgaris, which is known to respond to both conspecific and heterospecific PSF, as a model species.

Finally, I will conclude with a general discussion and synthesis of my findings (Chapter 8), by contrasting the results of the different studies with each other and with other work in the field. In addition, I will outline further research questions and identify issues for practical implementation of the results. 
CHAPTER 2

Soil inoculation steers restoration of terrestrial ecosystems

E. R. Jasper Wubs, Wim H. van der Putten, Machiel Bosch, and T. Martijn Bezemer

Nature Plants (2016) 2: 16017 


\begin{abstract}
Many natural ecosystems have been degraded due to human activities (Vitousek et al. 1997, Hobbs and Harris 2001) and need to be restored in order to protect biodiversity. However, restoration can take decades and restoration activities are often unsuccessful (Eviner and Hawkes 2008), because of abiotic constraints (e.g. eutrophication, acidification) and unfavourable biotic conditions (e.g. competition or adverse soil community composition). A key question is what manageable factors prevent transition from degraded to restored ecosystems and what interventions are required for successful restoration (Hobbs and Harris 2001, Kulmatiski et al. 2006). Experiments have shown that the soil community is an important driver of plant community development (De Deyn et al. 2003, Kardol et al. 2006, Carbajo et al. 2011, Van der Putten et al. 2013), suggesting that manipulation of the soil community is key to successful restoration of terrestrial ecosystems (Eviner and Hawkes 2008, Harris 2009). Here we examine a large-scale, six-year old field experiment on ex-arable land and show that application of soil inocula not only promotes ecosystem restoration, but that different origins of soil inocula can steer the plant community development towards different target communities, varying from grassland to heathland vegetation. The impact of soil inoculation on plant and soil community composition was most pronounced when the topsoil layer was removed, whereas effects were less strong, but still significant, when the soil inocula were introduced into intact topsoil. Therefore, soil inoculation is a powerful tool to both restore disturbed terrestrial ecosystems and steer plant community development.
\end{abstract}

\title{
Introduction
}

Plants and their associated soil communities are tightly inter-linked and influence each other during successional changes in developing ecosystems (Van der Wal et al. 2006, Bauer et al. 2015). Several studies with grassland plant communities have shown that inoculation with late-successional soil communities can increase the performance of late-successional target plant species, at the expense of earlysuccessional ruderals (De Deyn et al. 2003, Kardol et al. 2006, Carbajo et al. 2011, Bauer et al. 2015). This suggests that through inoculation with late-successional soils the typically positive feedback (Kardol et al. 2006, Bauer et al. 2015) between late-successional plants and their soil organisms may be restored (Harris 2009). However, two important aspects of plant-soil community interactions have remained unexplored: first it has not yet been studied to what extent soil communities may also steer the direction of plant community development towards different target states, and second, how soil legacies affect soil inoculation 
success in the field is unknown. We define steering of community development as the ability to direct the species composition of communities towards different target states when starting from the same environmental conditions.

Intensive arable farming reduces diversity and simplifies food webs of soil communities (Kulmatiski et al. 2006, Tsiafouli et al. 2015), and creates legacy effects in soil that can benefit weedy plant species for a long time (Kulmatiski et al. 2006, Kulmatiski and Beard 2011). Soil legacies are due to changes in abiotic and biotic soil conditions, for example because of increasing amounts of nutrients and soil-borne enemies of crop plants (Kulmatiski et al. 2006). As part of restoration projects in Europe the soil legacy effects of arable land management are often diminished through removal of the organic topsoil down to the mineral layer underneath, which e.g. reduces soil fertility (Marrs 1993, Jaunatre et al. 2014). However, in many cases the ecosystems remain dominated by ruderal plant species even after the soil legacies have been removed (Kardol et al. 2009, Carbajo et al. 2011). This indicates that other constraints, such as seed availability and soil community composition, may not have been alleviated (Hobbs and Harris 2001, Harris 2009, Carbajo et al. 2011). Restoration sites where soil legacies have been altered by topsoil removal may be particularly well-suited for testing the impact of soil inoculation on restoration with and without legacy effects of former agricultural management.

We tested the possibility of community steering through application of soil inocula in the field by analysing a large-scale, well-replicated, soil inoculation experiment on soils that had been used for arable farming for several decades. Topsoil was removed from large (2-5 ha) spatially-separated locations. Within each of these replicate locations we inoculated two different soil inocula and established controls where no further management was implemented. We used soil inocula from two donor sites: (i) a dry heathland and (ii) a grassland that had been restored 24 years ago. After six years, we analysed plant and soil community composition, as well as abiotic soil conditions, in order to test the hypothesis that application of the different inocula would not only promote community development, but that it would also steer the development of the plant and soil community composition into the direction of the respective donor sites. We performed an additional mesocosm experiment in order to validate that soil inoculation effects would not be solely due to adding plant seeds. 


\section{Methods}

The experiment was carried out on a 160 ha former arable field (Reijerscamp, the Netherlands, GPS: $52.015^{\circ} \mathrm{N}, 5.777^{\circ} \mathrm{E}$ ), which had been used to cultivate crops for almost six decades. Reijerscamp is situated on coarse Pleistocene sand (Gleyic Placic Podzol, derived from a sandur: a glacial outwash plain) with gravel and an organic top layer of $30-35 \mathrm{~cm}$, sometimes up to $50 \mathrm{~cm}$ thick. The site was grazed by cattle (25-30 cows throughout the year) upon completion of restoration measures and was further managed by periodic removal of tree seedlings (particularly Betula spp. and Prunus serotina).

Within the site, four separate experimental locations were selected. In each location the arable soil legacy was removed from $2-5 \mathrm{ha}$, by excavating the organic topsoil down to the mineral soil below $(50-70 \mathrm{~cm}$ depth). In the excavation areas we established three treatments. We inoculated soil from two different nearby sources, a heathland and a grassland ( $\mathrm{H}$ and $\mathrm{G}$, respectively), in large treatment plots (on average $0.5 \mathrm{ha}$ ) in each of the four locations and control plots $(\mathrm{C})$ were established where no further treatments were executed. In addition, in order to test the impact of the organic top-layer on inoculation success, we also inoculated plots of similar size with grassland soil outside the excavations and created controls there (i.e. without topsoil removal and soil inoculation). Hence, in total there were five experimental treatments carried out in each of the four locations (i.e. $\mathrm{N}=5$ $\mathrm{x} 4=20$ plots). The soil inocula were obtained from two nearby ( $<5 \mathrm{~km}$ distance) sites: one a grassland that had been under restoration for 24 years (Dennekamp, GPS: $52.029^{\circ} \mathrm{N} ; 5.801^{\circ} \mathrm{E}$ ) and an old dry heathland (Doorwerthse Heide, GPS: $51.995^{\circ} \mathrm{N} 5.778^{\circ} \mathrm{E}$ ). The grassland soil was inoculated at a rate of $\pm 2.5 \mathrm{~L} \mathrm{~m}^{-2}$ and heathland at a rate of $\pm 1.0 \mathrm{~L} \mathrm{~m}^{-2}$, with a commercial manure spreader, which resulted in a thin layer $<1 \mathrm{~cm}$.

Six years after the treatments had been implemented we conducted a field sampling campaign (July 2012, micro-arthropods: Sept 2012). To account for small-scale heterogeneity, we placed a randomly oriented transect across the centre of each of the 20 plots. Each transect consisted of five square subplots $\left(1 \mathrm{~m}^{2}\right)$ each separated by five meters from the next subplot (total transect length: $25 \mathrm{~m}$ ). In every subplot we analysed the composition of the plant and soil community and a number of abiotic soil parameters (see Supplementary Methods S2.1). The same sampling was conducted in the two donor sites (July 2013) except that no data on microarthropods were collected. The two donor sites were each a priori divided into four areas of equal size using geographical stratification, and one transect 
was placed randomly within each area. In the heathland the selected positions of transects were slightly adjusted during sampling in the field to ensure that areas where sods had recently been cut as part of the normal management were avoided.

We analysed the data using linear mixed models for univariate response data including random effects to account for the hierarchical sampling design. We explicitly modelled heteroscedasticity in the residuals using generalized least squares and post-hoc comparisons were corrected for multiple testing using the false-discovery rate. We used non-metric dimensional scaling (NMDS) to visualize differences in community composition and tested for significant differences among treatments using multiple-response permutation procedures (MRPP).

Additionally, we conducted a mesocosm experiment with mineral subsoil collected from the Reijerscamp at $>70 \mathrm{~cm}$ depth inoculated with soil from the two donor sites (9:1 w:w). Separate soil inocula were collected from each transect in the donor fields and used as replicates. We placed a 2-cm layer of sterile sand on top of the soils in order to prevent germination of seeds still present in the inoculum and sowed a standardized seed mixture of 30 species (Table S2.1) to ensure equal seed availability. After 30 weeks of growth we recorded the cover of all species in the treatments. As the mesocosm experiment setup was qualitatively the same as the design of the field experiment we analysed the data in the same way.

A detailed description of sample processing methods and data analysis can be found in the Supplementary Methods S2.1.

\section{Results}

Soil inoculation altered both plant- and soil community composition profoundly (Fig. 2.1, S2.1, S2.2, Table S2.2, S2.3). The composition of the plant communities in plots inoculated with heathland and grassland soils differed markedly from each other (Fig. 2.1). The cover of both grassland and heathland target species were promoted by both inocula, although the heathland species responded most strongly to the heathland inoculum (Fig. 2.2a-d). Both early- and mid-successional species remained unaffected by soil inoculation. Moreover, inoculation led to plant communities that diverged from the controls in the direction of their respective donor community (Fig. 2.1b, S2.3; Table S2.4). This shows that, depending on the origin of the soil inoculum, the plant community development can be steered towards either a grassland or a heathland. 

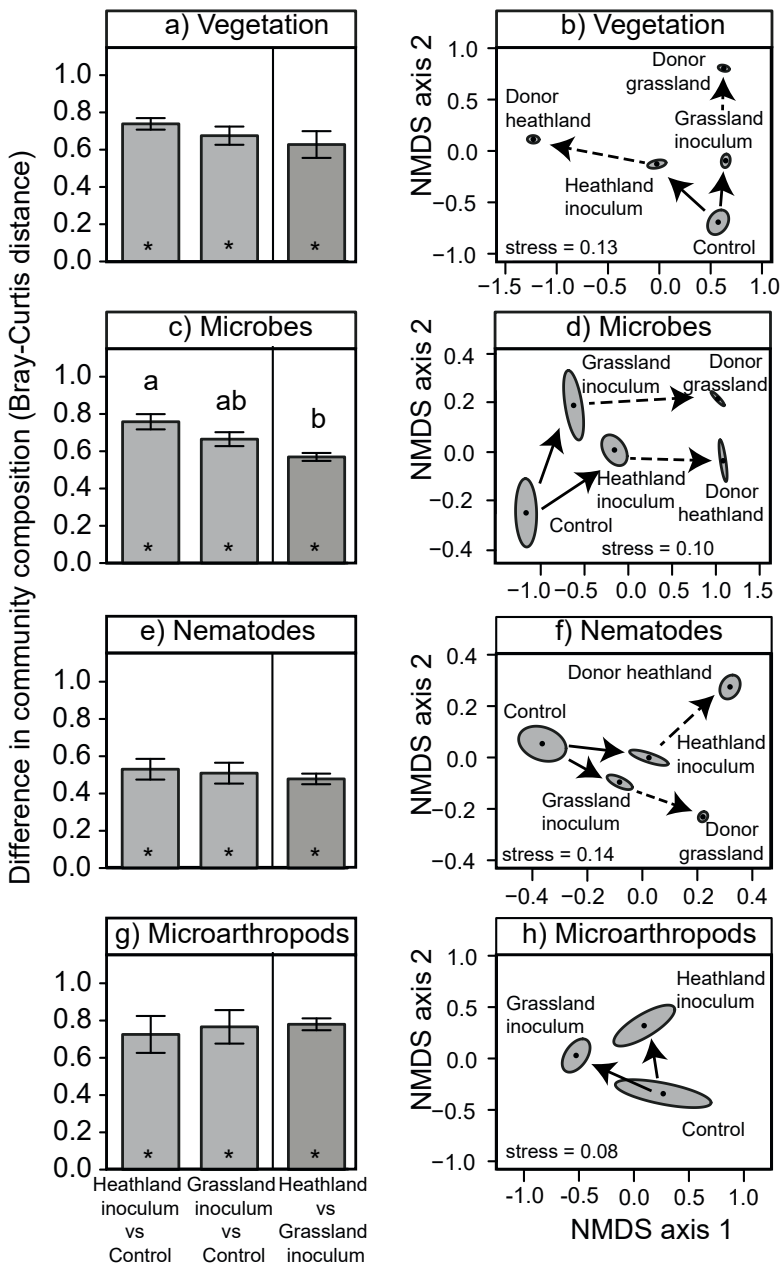

\section{Fig. 2.1. The effect of soil inoculation with two different soil inocula on plant and soil community composition in the field after topsoil removal.}

The left column (panel a, c, e, g) shows the difference in community composition (Bray-Curtis distance; range $0-1, \operatorname{mean} \pm$ SE.) of the plant and soil community relative to control (light bars) and the difference between plots treated with the two different inocula (heathland and grassland; dark bars). Stars indicate significant differences from zero (i.e. among the two communities compared per bar), while different letters above the bars indicate significant differences among means (see Table S2.2). The right column shows non-metric dimensional scaling (NMDS) plots (panels b, d, $\mathrm{f}, \mathrm{h}$ ) of community composition in the three experimental treatments and the two donor sites (dots are means, ellipses SEs). Differences among treatments were significant in all cases (Table S2.3). The solid arrows indicate the direction of the effect of soil inoculation on community composition. The dotted arrows indicate the distance in community composition between the inoculated plots and their respective donor community. Stress values for each NMDS analysis are given. Stress is a lack of fit statistic (zero means perfect fit) indicating to what extent the two-dimensional plot represents the multidimensional differences in community composition (Bray-Curtis distances) in the raw data. 


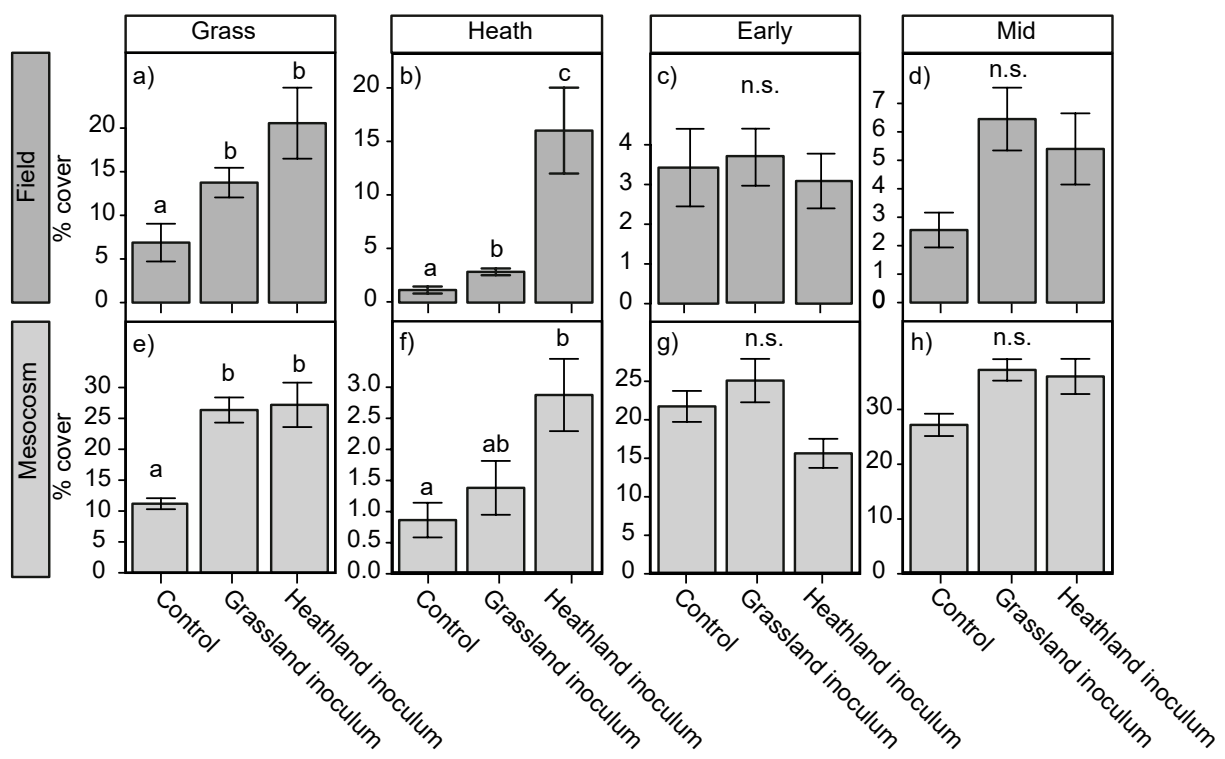

Fig. 2.2. Changes in abundance of plant species groups in response to soil inoculation.

Shown are responses of target species for grassland (a, e) and heathland (b, f) respectively, as well as early- (c, g) and mid-succession (d, h) species. The top panels represent data from the field experiment (a-d), while the bottom panels are from the mesocosm experiment (e-h). Different letters indicate significant differences, for the overall analysis see Table S2.9. See Fig S2.2 for species membership to the different successional groups.

Soil inoculation also drove soil community composition towards that of the donor sites (Fig 2.1d, f, S2.3; Table S2.4). Inoculation with heathland soil significantly increased the biomass of both bacteria and fungi (Table S2.5A). Both grassland and heathland soil inocula increased the abundance of arbuscular mycorrhizal fungi and the total number of nematodes (Table S2.5A). Heathland inoculum enhanced the diversity of springtails (Collembola) and mites (Acari), but not their total numbers (Table S2.5A). Most soil abiotic conditions were not affected by soil inoculation, except that in the inoculated soils percentage organic matter increased from 1.2 to $1.9-2.5 \%$ (Table S2.6A).

In the mesocosm experiment, we inoculated a common field soil (mineral subsoil from the field site) with soil inocula from the two donor sites and established controls where no inoculum was added. We subsequently sowed a standardized mixture of 30 plant species (Table S2.1) in all treatments and recorded the percent cover of all plant species after 30 weeks of growth. The species mixture consisted of 10 representatives each for the early-, mid- and late-successional stages on sandy- 

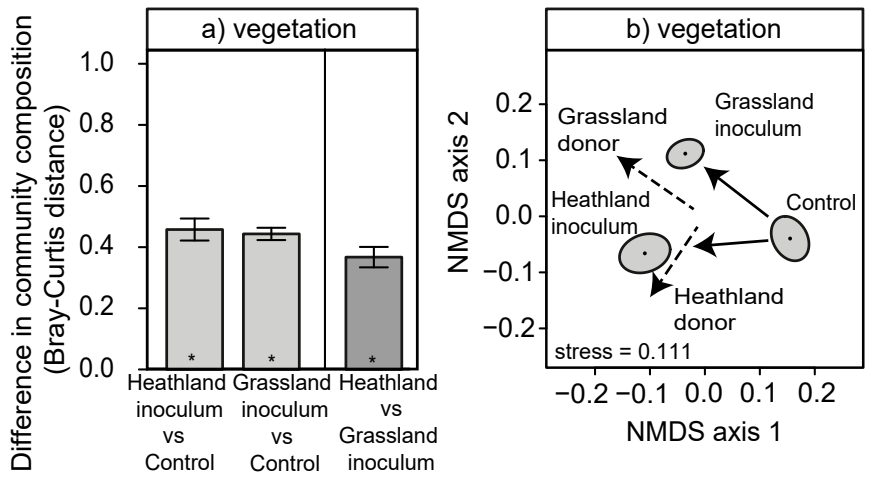

Fig. 2.3. Differences in plant community composition from the control due to inoculation $(10 \%$ w:w) with grassland and heathland soil inocula respectively in a controlled mesocosm experiment.

To alleviate differences in seed availability, inoculated and non-inoculated containers were sown with a standardized seed mixture of 30 plant species (Table S2.1) and a layer of sterilized sand was placed over the mesocosm soil to prevent germination from the seed bank. a) The difference in community composition (Bray-Curtis distance; range $0-1, \operatorname{mean} \pm \mathrm{SE}$ ) of the vegetation relative to control (light bars) and the difference between mesocosms treated with the two different inocula (heathland and grassland; dark bar) are shown. Stars indicate significant difference in community composition (i.e. different from zero; Table S2.7). b) Visualization of the differences in plant community composition (dots are means, ellipses SEs) using non-metric multidimensional scaling (NMDS; MRPP Pseudo-F $=4.1, \mathrm{p}=0.001$ ). For each of the mesocosms their similarity to the two donor sites was calculated, and the direction of increasing similarity is plotted using dashed arrows. The solid arrows indicate the direction of the effect of soil inoculation on community composition.

soils in the region. Soil inoculation with heathland and grassland soils shifted the composition of the sown plant communities in different directions (Fig. 2.3, $\mathrm{S} 2.2 \mathrm{~b})$. Plant species representative of the target communities benefitted from soil inoculation, while early- and mid-succession species remained unaffected (Fig. 2.2e-h). Furthermore, the two soil inocula led to plant communities that each shifted in the direction of their respective donor community (Fig. 2.3). Therefore soil inoculation still steered plant community development into the direction of the vegetation composition of the donor sites, even when differences in plant propagules were excluded.

Finally, we compared the effectiveness of soil inoculation between plots with and without intact topsoil. For every treatment plot inoculated with grassland soil, we had an adjacent replicate plot where the same soil inoculum was introduced in intact topsoil with a full arable legacy. There was no such treatment for heathland soil. The plots with intact topsoil had higher soil organic matter content and nutrient availability, as well as a higher abundance of bacteria, fungi, nematodes 


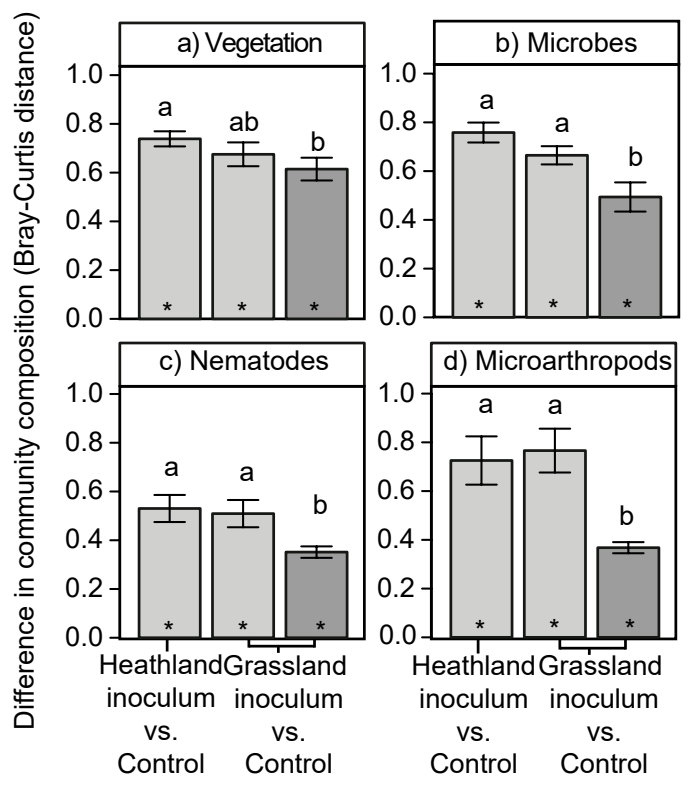

Fig. 2.4. Comparison of the effect of soil inoculation on plant (a) and soil community (b-d) composition in plots with (light bars) and without (dark bars) topsoil removal.

The extent to which the communities were different from the control (mean $\pm S E$, Bray-Curtis dissimilarity; range 0-1) after inoculation with soil from a heathland and a grassland is shown. Data in the light bars show the effect of inoculation with heathland and grassland soil after removal of the topsoil, data in the dark bars is the effect of inoculation with grassland soil in plots where the topsoil had not been removed. Stars indicate significant differences from zero (which equals no effect), different letters indicate significant differences among means within panels (see Table S2.8 for analysis).

and micro-arthropods (Table S2.5, S2.6). Nevertheless, soil inoculation still had a significant effect on both plant and soil community composition of the topsoil (Fig. 2.4, Table S2.8a,b). In the inoculated plots, plant species composition was closer to the donor vegetation than the respective controls, irrespective of topsoil removal (Fig. S2.4). However, we found that soil inoculation influenced plant and soil community composition less strongly in plots with than without intact topsoil, particularly for the soil community (Fig. 2.4, Table S2.8c).

\section{Discussion}

Our results show that soil inoculation can promote ecological restoration in the field. This has been suggested before (Harris 2009), however, most field tests have been done with soil transplants (Vécrin and Muller 2003, Antonsen and Olsson 2005, Box et al. 2011, Pywell et al. 2011, Jaunatre et al. 2014, Bulot et al. 2014) 
and not with soil inocula (Kardol et al. 2009, Hamman and Hawkes 2013), which introduce considerably lower amounts of propagules. Moreover, we demonstrate that depending on the origin of the soil inoculum the restoration site could be steered to become either grassland or heathland. Glasshouse studies have indicated that the soil community is an important driver of plant community composition, and that late-successional plant species experience positive soil feedbacks, while ruderal species tend to have negative feedbacks (De Deyn et al. 2003, Kardol et al. 2006, Bauer et al. 2015). However, for successful restoration plant propagules from the target community also need to be present, as many target species are dispersal limited (Bullock 1998, Bakker and Berendse 1999). Our mesocosm experiment shows that when differences in seed availability are alleviated, soil inoculation can still steer vegetation composition. Therefore, differences in plant community composition in the field were not solely the result of co-introducing plant propagules with the inoculum. We suggest that the co-introduction of both plant propagules and their associated soil biota restored the typically positive feedbacks among late-successional plants and their associated soil biota (De Deyn et al. 2003, Kardol et al. 2006, Rowe et al. 2007). These feedbacks are thought to be major drivers of succession, suggesting that the positive effects of a single soil inoculation on the plant community may persist for prolonged periods of time.

Our study shows that soil inoculation in the field may both promote nature restoration and steer the community development depending on the origin of the soil inoculum. In our experiment the inocula application rates were quite different among grassland $\left(2.5 \mathrm{~L} \mathrm{~m}^{-2}\right)$ and heathland inocula $\left(1.0 \mathrm{~L} \mathrm{~m}^{-2}\right)$. However, we found the strongest effects in the treatment with the lowest amount of soil inoculum (heathland-inoculum). Therefore, we do not think that this difference was a major factor driving the results. Other studies have shown that inoculation of soils with arbuscular mycorrhizal fungi (AMF) can also promote the performance of latesuccessional plants over ruderal species and that local AMF strains outperform commercial strains (Rowe et al. 2007, Paluch et al. 2013, Emam 2016). However, several studies have argued that the transfer of whole soil communities is more effective than the addition of individual species or strains (Rowe et al. 2007, Hoeksema et al. 2010, Paluch et al. 2013, Emam 2016). In addition, among the soil transplantation experiments in the field (Vécrin and Muller 2003, Antonsen and Olsson 2005, Kardol et al. 2009, Box et al. 2011, Pywell et al. 2011, Hamman and Hawkes 2013, Jaunatre et al. 2014, Bulot et al. 2014), large-sized treatments tend to be more successful at restoring native plant communities than soil transfers applied at a small spatial scale. Thinly spread soil inocula, as used in our study, can restore 
much larger areas than when entire soil layers are translocated (Vécrin and Muller 2003, Box et al. 2011, Pywell et al. 2011, Bulot et al. 2014), which may enhance the feasibility of this procedure as soil collection will disrupt current nature areas.

The success of inoculation in the field also depended on the presence of a legacy of arable cultivation: the impact of inoculation with grassland soil was greater when the topsoil was removed than when added to intact topsoil. The treatment plots with and without arable soil legacy differed substantially in their abiotic conditions, as well as soil community composition. It is, therefore, not possible to disentangle the exact causes in this experiment. However, it has been established that both low soil nutrient conditions (De Deyn et al. 2004, Carbajo et al. 2011) and reduced competition from resident soil communities (Van Elsas et al. 2012) can enhance the impact of soil inoculation. Importantly, however, the effect of soil inoculation on community composition was still clearly present even in the intact topsoil. Further research should, therefore, test whether carefully chosen soil inocula may reduce the need for expensive topsoil removal, which has considerable environmental costs (Jaunatre et al. 2014).

We conclude that under field conditions soil inoculation can steer the course of community development on ex-arable land, irrespective of topsoil removal, but that effects are greatest when removing the topsoil prior to soil inoculation. Depending on the origin of the soil inoculum, the composition of the plant community in the recipient site was directed towards a heathland or a grassland vegetation. Based on our results we suggest that manipulation of soil communities through soil inoculation is a powerful tool for the restoration of degraded terrestrial ecosystems. 


\section{Supplementary information}

\section{Supplementary methods}

\section{Plot sampling \& sample processing.}

Within each subplot $\left(1 \mathrm{~m}^{2}, 5\right.$ per transect $)$ all vascular plants were identified (nomenclature following Van der Meijden 2005) and their percent cover estimated. Within each subplot 36 soil cores of $\varnothing 1.1 \times 15 \mathrm{~cm}$ deep were taken from a six by six sample grid spread evenly across the plot and pooled to be analysed for soil abiotic and biotic variables. Soil samples were transported cool and stored at $4{ }^{\circ} \mathrm{C}$ until further processing within two weeks. The pooled soil cores were first sieved using a $1 \mathrm{~cm}$ mesh and then carefully homogenized. Approximately 100 $\mathrm{g}$ fresh soil was stored for nematode extraction (see below) for plots 2 and 4 of each transect. The remaining sample was sieved again using a $4 \mathrm{~mm}$ mesh and approximately $10 \mathrm{~g}$ was subsampled to determine soil organic matter content by loss on ignition for $24 \mathrm{~h}$ at $430^{\circ} \mathrm{C}$. From the remaining sample: about $50 \mathrm{ml}$ fresh soil was taken for PLFA analysis and stored at $-20{ }^{\circ} \mathrm{C}$ until analysis. A subsample of $200 \mathrm{~g}$ was collected for soil chemical analysis and dried for $7 \mathrm{~d}$ at $40^{\circ} \mathrm{C}$.

\section{Soil chemistry.}

Soil acidity was measured in a 1:2.5 dry-soil:demi-water (w:v) solution upon shaking for two hours at $250 \mathrm{rpm}$. Mineral $\mathrm{N}$ was extracted by shaking $10 \mathrm{~g}$ dry soil in a $1 \mathrm{M} \mathrm{KCl}(1: 5 \mathrm{w}: \mathrm{v})$ solution for two hours at $250 \mathrm{rpm}$. The suspensions were centrifuged for $10 \mathrm{~min}$ at $10000 \mathrm{rpm}$ and $\mathrm{NO}_{3}-\mathrm{N}$ and $\mathrm{NH}_{4}-\mathrm{N}$ content was determined in the supernatant using a QuAAtro39 AutoAnalyzer (SEAL Analytical, Southhampton, UK). Total extractable phosphate content was determined using P-Olsen extraction (Olsen et al. 1954) with $0.5 \mathrm{M} \mathrm{NaHCO}_{3}$ and spectrophotometric detection ( $\lambda=720 \mathrm{~nm}$; molybdenum blue colouring reagent) using a Synergy HT Multi-Mode Microplate Reader (BioTek Instruments Inc., Winooski, VT, USA).

\section{Microbial community analysis.}

The abundance of bacteria and (arbuscular mycorrhizal) fungi was analysed by phospho- (PLFA) and neutral (NLFA) lipid fatty acid extraction (Boschker 2004). Before extraction the $50 \mathrm{ml}$ soil subsamples were freeze-dried. Briefly, total lipids were extracted from $3 \mathrm{~g}$ of soil using Blight $\&$ Dyer extraction, and separated into polar and non-polar fractions using silicic acid column chromatography. The lipids were transesterified using mild alkaline methanollysis to recover the lipids as methyl esters in hexane. Separation and detection of fatty acid methyl 
esters was performed on a gas chromatograph (7890A, Agrilent Technologies Inc., Santa Clara, CA, USA) equipped with a flame ionization detector and a HP-5MS column (length $60 \mathrm{~m}$, diameter $0.250 \mathrm{~mm}$, film $0.25 \mu \mathrm{m}$ ). Retention times of the fatty acids were compared to those of a FAME 37 and BAME 26 component standard mixes (Supelco Analytical, Bellefonte, PA, USA). Peak areas were converted to lipid weights using methyl nonadecanoate fatty acid (19:0) as the internal standard, which was added before methylation. PLFA and NLFA peaks were assigned to taxonomic groups based on Frostegård et al. 1993, Frostegård and Bååth 1996, Ruess and Chamberlain 2010, Ngosong et al. 2012). PLFA compounds were used as general microbial $(2 \mathrm{OH}-\mathrm{C} 10: 0,2 \mathrm{OH}-\mathrm{C} 12: 0,3 \mathrm{OH}-$ C12:0, 2OH-C14:0, 2OH-C16:0), bacterial (iC14:0, iC15:0, aiC15:0,C15:0,

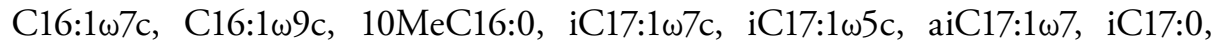
aiC17:0, cy-C17:0,C17:0, 10MeC17:0, 10MeC18:0, iC18:0, cy-C19:0), and fungal

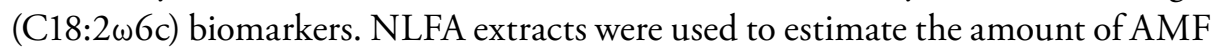
biomass (marker 16:1w5). All compounds that could be assigned to a microbial taxon were used in analyses of community composition (see below). The total microbial biomass was calculated as the sum of all microbial, bacterial and fungal markers. However, the NLFA based AMF marker was excluded as the extraction efficiencies of the PLFA and NLFA extractions differ substantially. The ratio of fungal to bacterial markers (PLFA only) was used to characterize the microbial community (Bardgett et al. 1996). In a few cases ( $\mathrm{n}=7$; only for TSR-Control plots) no bacterial biomass could be detected using this method and they were excluded from these calculations

\section{Soil invertebrates sampling.}

Nematodes were extracted from $100 \mathrm{~g}$ fresh soil using Oostenbrink elutriators (Oostenbrink 1960), heat-killed at $90^{\circ} \mathrm{C}$, and fixed in $4 \%$ formaldehyde solution. Subsequently the total number of nematodes was counted and at least 100 were identified to family or, when possible, genus level (nomenclature following Bongers 1988). In four cases where there were less than 100 nematodes, all nematodes were identified. In September 2012, soil microarthropods were collected using soil cores of $\varnothing 9.2 \times 5 \mathrm{~cm}$ depth, which were collected from subplots two and four of all transects. They were directly extracted using Tullgren funnels (Van Straalen and Rijninks 1982) and all animals were stored in 70\% ethanol. From the samples all adult mites (Acari) and springtails (Collembola) were identified to genus or, when possible, species level (nomenclature following Gisin 1960, Zimdars and Dunger 1994, Fjellberg 1998, 2007, Bretfeld 1999). 


\section{Glasshouse soil inoculation experiment.}

In order to test if the soil community was a driver of plant community composition we conducted a glasshouse experiment. Common background soil was collected from Reijerscamp, where a fresh pit of $6 \mathrm{~m}^{2}$ was dug and mineral, white, sandy soil was collected from $\geq 70 \mathrm{~cm}$ depth to mimic the field experiment. The soil was sieved ( $5 \mathrm{~mm}$ mesh) and analysed for chemical and microbial composition as above ( $\mathrm{n}=5$, mean \pm SEM: $6.27 \pm 0.02 \mathrm{pH}, 0.05 \pm 0.02 \mathrm{mg} / \mathrm{kg} \mathrm{NO}-\mathrm{N}, 0.33 \pm 0.16 \mathrm{mg} / \mathrm{kg}$ $\mathrm{NH}_{4}-\mathrm{N}, 3.35 \pm 1.21 \mathrm{mg} / \mathrm{kg} \mathrm{PO}_{4}-\mathrm{P}$, microbial community biomass $0.084 \pm 0.077 \mu \mathrm{g}$ PLFA/g soil). Soil inocula were collected from the two donor sites along the same transects as used before to characterize the donor sites (see Methods). Soil was collected from the same positions (retraced using GPS, error $\pm 5 \mathrm{~m}$ ) as subplots 2,3 and 4 in the original transects after removal of the vegetation in the first week of July 2014. In each plot about $5 \mathrm{~kg}$ of inoculum $(0-15 \mathrm{~cm}$ depth) was collected, sieved with $0.5 \mathrm{~cm}$ mesh and homogenized. Consequently, we collected 12 inocula from three subplots in each of four transects per donor site, which were kept separate throughout the experiment. The inocula were mixed with the background soil (1:9 w:w) and placed in square containers of $17 \times 17 \times 17 \mathrm{~cm}$, so that each container received $4 \mathrm{~kg}$ of soil. In addition, 12 containers were filled only with background soil in order to serve as control without inoculum. In total there were 36 containers $(\mathrm{N}=3$ treatments $\mathrm{x} 4$ transects $\mathrm{x} 3$ subplots/transect = 36). A $2-\mathrm{cm}$ layer of sterilized ( $>25 \mathrm{KGray}$ gamma radiation, Isotron, Ede, the Netherlands) background soil was added to all containers on top of the soil to prevent any germination of seeds from the inocula. Subsequently, each container was sown with a standardized seed mixture of 30 plant species (Table S2.1), with 10 representatives each of early, middle and late successional stages (Schaminée et al. 1996, Kardol et al. 2006, Van de Voorde et al. 2011). As the probability of establishment typically increases with seed weight (Jakobsson and Eriksson 2000), the same seed weight ( $16 \mathrm{mg}$, in total $\pm 0.5 \mathrm{~g}$ ) was sown per container for each species, except for the relatively large-seeded species Vicia cracca for which always two seeds were added ( $24 \mathrm{mg} / \mathrm{seed}$ ). Four species (Viola arvensis, Elytrigia repens, Achillea millefolium, and Nardus stricta) required cold stratification and were placed in a cold room at $4{ }^{\circ} \mathrm{C}$, in moistened paper bags for two weeks prior to sowing. The containers were placed in the glasshouse at 16:8 h day:night, 21:16 ${ }^{0} \mathrm{C}, \geq 60 \%$ relative humidity, natural light supplemented with $600 \mathrm{~W}$ metalhalide lamps, 1 per $4 \mathrm{~m}^{-2}$, approx. $225 \mu \mathrm{mol}$ light quanta $\mathrm{m}^{-2} \mathrm{~s}^{-1}$ at plant level. Containers were placed in randomized locations stratified by field transect. The containers were watered three times per week and allowed to grow for 30 weeks. Subsequently the percent cover of all plant species was recorded. In only a few 
cases non-sown species germinated and they always had low abundance of $\leq 2 \%$ cover. As growth was slow $50 \mathrm{ml} 0.25$ strength Hoagland nutrient solution was added to each container in weeks 16 and 18.

\section{Data analysis.}

To test if soil inoculation shifted community composition of soil biota and plants in the field we calculated the difference in community composition between plots treated with soil inocula and control plots as the Bray-Curtis distance (Bray and Curtis 1957), which is a robust measure of dissimilarity that is commonly used in community ecology (Austin 2013). This index is bound between zero and one, with zero indicating complete similarity in relative abundance of all species, while one indicates that no species are shared between the samples (complete turnover). Species abundances were log-transformed, and the microbial community biomarkers were rescaled by their standard deviation to account for the difference in extraction efficiencies among PLFA and NLFA extractions. In order to test to what extent the communities that resulted from the inoculation with different soil inocula (grassland vs. heathland inoculum) diverged we also calculated the Bray-Curtis distance between them. Community distances were calculated on a sample (=subplot) basis. The three groups of dissimilarities were analysed in linear mixed models per taxonomic community. The type of comparison (three levels: heathland-inoculum vs. control, grassland-inoculum vs. control, heathlandvs. grassland-inoculum) was used as a fixed effect, while the sampling location (i.e. excavation) and transect ID were used as nested random effects. In case of heteroscedasticity separate variances were modelled per type of comparison using generalized least squares (Pinheiro and Bates 2000, Zuur et al. 2009). Post-hoc comparisons were corrected for multiple testing using the false-discovery rate (Benjamini and Hochberg 1995). Within each model the significance of individual model terms was inspected to test if each comparison differed significantly from zero (Fox et al. 2015).

In order to test if soil inoculation was also effective when the arable soil legacy was left intact, Bray-Curtis distance between treated and control plots in areas without topsoil removal were calculated as before. These data were analysed using a linear mixed model as before, but including the calculated dissimilarities of plots treated with both inocula within topsoil removal areas for comparison. Consequently, in this analysis there was a three-level 'type of comparison' fixed effect as well, i.e. both inocula vs. control on areas with topsoil removal, and grassland-inoculated 
vs. control in areas without topsoil removal). These analyses were conducted for each taxonomic community.

The similarity of each of the three treatments (control, grassland-, and heathlandinoculated) to both donor sites were calculated as the Sørensen similarity (which for relative abundance data $=1-$ Bray-Curtis distance). The calculated similarities were analysed by linear mixed models with the same structure as before, but with soil treatment (control, grassland-, and heathland-inoculated) as fixed effect. These models were analysed separately per taxonomic community and donor type.

To visualize differences in community composition we conducted non-metric multidimensional scaling (NMDS), which is currently considered to be the most robust ordination method (Austin 2013, Jaunatre et al. 2014). Ordinations were performed on Bray-Curtis distance of log-transformed abundances of the three soil treatments and the two donor sites simultaneously per taxonomic community. Each NMDS analysis was run twice to check for consistency. Analysis of both solutions indicated that the runs produced similar ordinations for all communities (small Procrustes errors, data points did not shift qualitatively in position). Differences in community composition were tested using permutation tests (multiple-response permutation procedure, MRPP). To account for the hierarchical sampling design species abundances were averaged per transect and whole transects (= plots) were permuted (999 permutations) within sampling locations.

Both community distance and NMDS analyses for the plant community in the mesocosm experiment were conducted in the same way as those for the field experiment, since the sampling design was essentially the same (four locations per treatment sampled, three subplots per transect, $\mathrm{N}=3 * 4 * 3=36$ ). Species responses in the field and mesocosm experiments were further analysed by grouping them into early-, mid-, and late-successional species and late-successional species were further divided into species representative of the grassland and heathland targets respectively (following Schaminée et al. 1996, 1998, Kardol et al. 2006, Kardol 2007, Van de Voorde et al. 2011). Univariate analyses on abiotic, soil biotic and vegetation properties were analysed with the same linear mixed model structure as the similarity analyses in the field experiment. For simplicity, count data were also analysed using LMMs with Gaussian errors as the residuals were close to a normal distribution after log-transformation. 
All calculations and analyses were conducted in $\mathrm{R}$ (version 2.15.3, $\mathrm{R}$ Core Team 2016). Linear mixed models were fitted within the package nlme (3.1-108, Pinheiro et al. 2016), multiple comparisons using the multcomp package (1.2-18, Hothorn et al. 2008), and NMDS and MRPPs were conducted in the vegan package (2.08, Oksanen et al. 2016). 
Table S2.1. List of plant species used in the mesocosm experiment.

\begin{tabular}{|c|c|c|c|c|}
\hline Scientific name & $\begin{array}{c}\text { mono-l } \\
\text { dicot }\end{array}$ & $\begin{array}{c}\text { Succes- } \\
\text { sional stage* }\end{array}$ & $\begin{array}{l}\text { Average seed } \\
\text { mass }(\mathrm{mg})^{* *}\end{array}$ & $\begin{array}{l}\text { Estimated \# } \\
\text { seeds added }\end{array}$ \\
\hline Achillea millefolium & $\mathrm{d}$ & Mid & 0.23 & 70 \\
\hline Anthoxanthum odoratum & $\mathrm{m}$ & Mid & 0.60 & 27 \\
\hline Arnica montana & d & Late & 1.29 & 12 \\
\hline Briza media & $\mathrm{m}$ & Mid & 0.59 & 27 \\
\hline Calluna vulgaris & $\mathrm{d}$ & Late & 1.46 & 11 \\
\hline Campanula rotundifolia & $\mathrm{d}$ & Late & 0.64 & 30 \\
\hline Crepis capillaris & $\mathrm{d}$ & Early & 0.36 & 44 \\
\hline Elytrigia repens & $\mathrm{m}$ & Early & 2.51 & 6 \\
\hline Festuca filiformis & $\mathrm{m}$ & Late & 0.39 & 41 \\
\hline Galium verum & $\mathrm{d}$ & Late & 0.59 & 27 \\
\hline Hieracium pilosella & $\mathrm{d}$ & Late & 0.24 & 68 \\
\hline Holcus lanatus & $\mathrm{m}$ & Mid & 0.51 & 32 \\
\hline Hypochaeris radicata & $\mathrm{d}$ & Late & 0.80 & 20 \\
\hline Leucanthemum vulgare & $\mathrm{d}$ & Mid & 0.54 & 30 \\
\hline Lolium perenne & $\mathrm{m}$ & Early & 2.16 & 7 \\
\hline Lotus corniculatus & $\mathrm{d}$ & Mid & 2.03 & 8 \\
\hline Luzula campestris & $\mathrm{m}$ & Late & 0.95 & 17 \\
\hline Myosotis arvensis & $\mathrm{d}$ & Early & 0.93 & 17 \\
\hline Nardus stricta & $\mathrm{m}$ & Late & 0.62 & 26 \\
\hline Plantago lanceolata & $\mathrm{d}$ & Mid & 2.39 & 7 \\
\hline Poa trivialis & $\mathrm{m}$ & Early & 0.38 & 42 \\
\hline Rumex acetosella & d & Early & 0.55 & 29 \\
\hline Tanacetum vulgare & $\mathrm{d}$ & Mid & 0.16 & 101 \\
\hline Thymus pulegioides & $\mathrm{d}$ & Late & 0.63 & 25 \\
\hline Trifolium dubium & $\mathrm{d}$ & Mid & 1.03 & 15 \\
\hline Trifolium pratense & $\mathrm{d}$ & Early & 2.00 & 8 \\
\hline Trifolium repens & $\mathrm{d}$ & Early & 1.70 & 9 \\
\hline Tripleurospermum maritimum & $\mathrm{d}$ & Mid & 0.50 & 32 \\
\hline Vicia cracca & $\mathrm{d}$ & Early & 24.36 & 2 \\
\hline Viola arvensis & $\mathrm{d}$ & Early & 0.82 & 20 \\
\hline
\end{tabular}

* Based on Schaminée et al. 1996, 1998, Kardol et al. 2006, Kardol 2007, Van de Voorde et al. 2011.

** Based on at least 100 seeds (range 100-323), fresh mass is reported. 

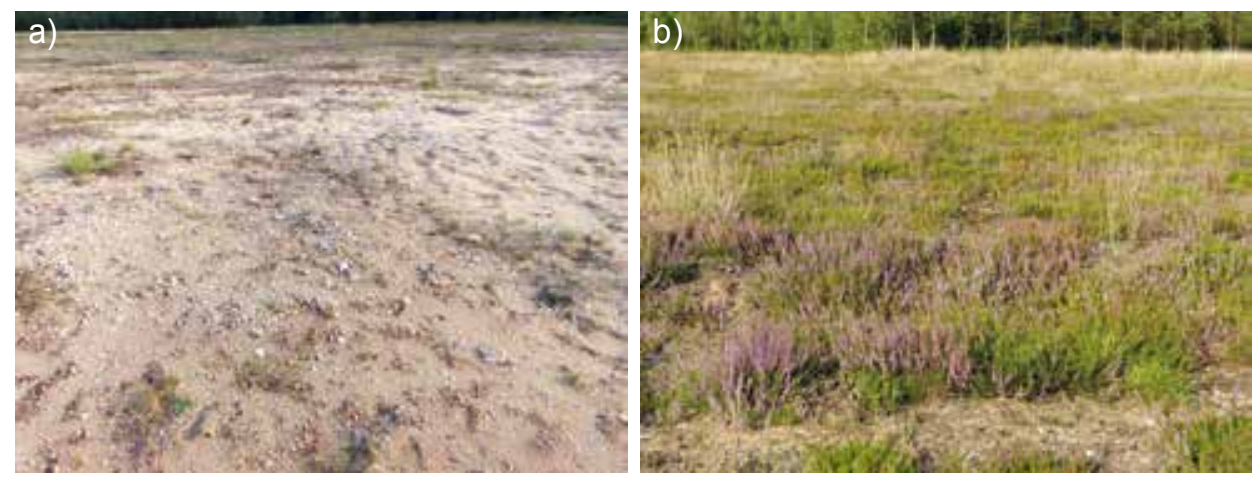

Fig. S2.1. Effect of inoculation with heathland soil on the plant community.

Photographs were taken in the field for two immediately adjacent treatment plots ( $\pm 30 \mathrm{~m}$ apart).

a) control plot without soil inoculation. b) plot inoculated with heathland soil. Both plots with topsoil removal, photographs were taken in August 2014, eight years after the start of the experiment.

Table S2.2. Statistical analyses for differences in community composition from the noninoculated control ( $\mathrm{H}$ vs. C and G vs. C) and between the heathland and the grassland inocula (H vs. G).

\begin{tabular}{|c|c|c|c|c|c|c|c|}
\hline \multirow[t]{2}{*}{ Response } & \multicolumn{2}{|c|}{$\begin{array}{l}\text { a) Test for overall } \\
\text { difference from no } \\
\text { effect (i.e. 0) }\end{array}$} & \multicolumn{3}{|c|}{$\begin{array}{l}\text { b) Per treatment tests for } \\
\text { difference from no effect (i.e. 0) }\end{array}$} & \multicolumn{2}{|c|}{$\begin{array}{l}\text { c) Test for among } \\
\text { treatment differences } \\
\text { in effect size }\end{array}$} \\
\hline & $\mathrm{F}_{3,6}$ & p-value & Treatment & $t_{6}$ & $\mathrm{p}$-value & $\mathrm{F}_{2,6}$ & p-value \\
\hline \multirow[t]{3}{*}{ Vegetation } & 53.3 & 0.0001 & $\mathrm{H}$ vs $\mathrm{C}$ & 11.4 & $<0.0001$ & 2.4 & 0.17 \\
\hline & & & G vc C & 10.5 & $<0.0001$ & & \\
\hline & & & $\mathrm{H}$ vs $\mathrm{G}$ & 11.0 & $<0.0001$ & & \\
\hline \multirow{3}{*}{$\begin{array}{l}\text { Microbial } \\
\text { community }\end{array}$} & 249.0 & $<0.0001$ & $\mathrm{H}$ vs $\mathrm{C}$ & 20.0 & $<0.0001$ & 5.8 & 0.04 \\
\hline & & & G vc C & 12.6 & $<0.0001$ & & \\
\hline & & & $\mathrm{H}$ vs $\mathrm{G}$ & 13.7 & $<0.0001$ & & \\
\hline \multirow[t]{3}{*}{ Nematodes } & 71.4 & $<0.0001$ & $\mathrm{H}$ vs $\mathrm{C}$ & 9.1 & 0.0001 & 0.7 & 0.52 \\
\hline & & & G vc C & 11.0 & $<0.0001$ & & \\
\hline & & & $\mathrm{H}$ vs $\mathrm{G}$ & 13.9 & $<0.0001$ & & \\
\hline \multirow{3}{*}{$\begin{array}{l}\text { Micro- } \\
\text { arthropods }\end{array}$} & 72.6 & $<0.0001$ & $\mathrm{H}$ vs $\mathrm{C}$ & 6.4 & 0.0007 & 0.1 & 0.92 \\
\hline & & & G vc C & 7.2 & 0.0004 & & \\
\hline & & & $\mathrm{H}$ vs $\mathrm{G}$ & 11.2 & $<0.0001$ & & \\
\hline
\end{tabular}


a) Field experiment

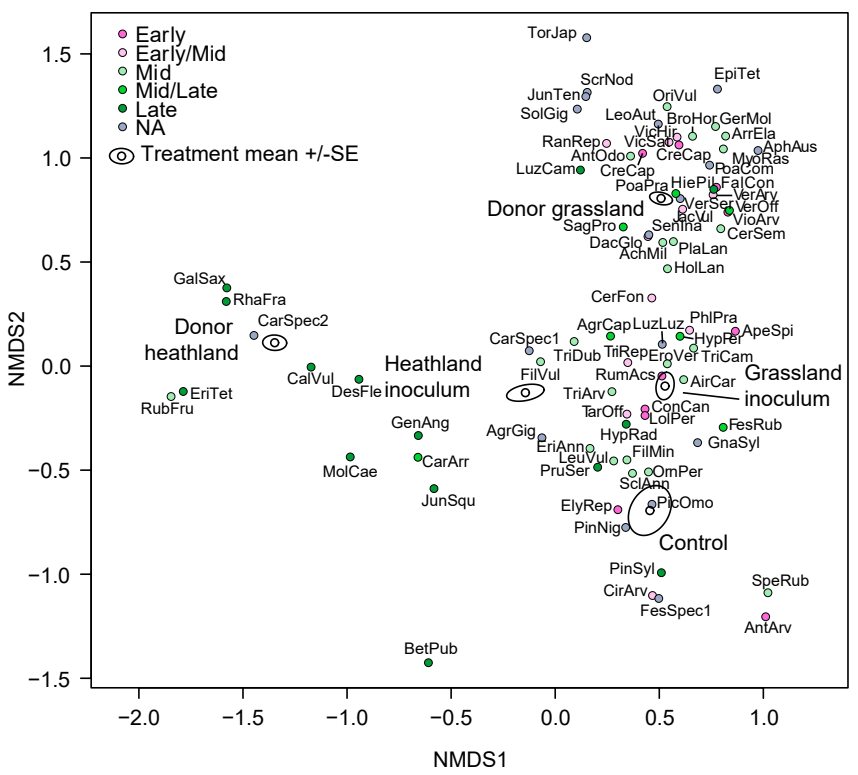

b) Mesocosm experiment

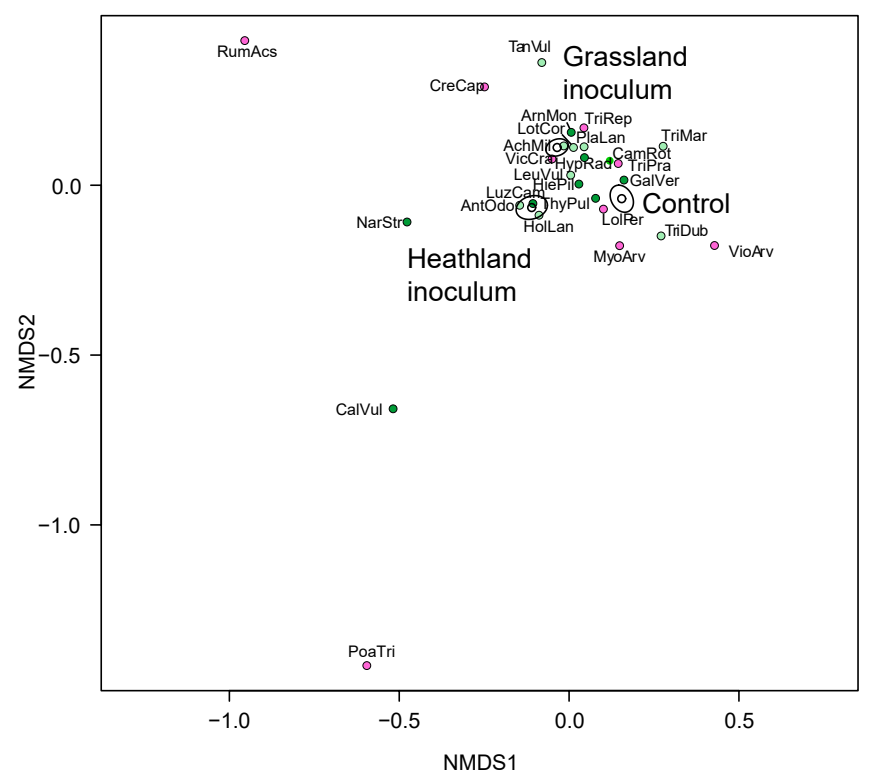

Fig. S2.2. Plant species composition in response to soil inoculation.

Vegetation composition as visualized using NMDS in a) the field (c.f. Fig. 2.1b) and b) the mesocosm experiment (c.f. Fig. 2.3b). Species were plotted by their NMDS scores and treatment means and SEs are presented as in Figs. 2.1b and 2.3b. Species abbreviations are: AphAus: Aphanes australis, AgrCap: Agrostis capillaris, AgrGig: Agrostis gigantea, AirCar: Aira caryophyllea, AntArv: 


\section{Fig. S2.2. caption continued.}

Anthemis arvensis, AntOdo: Anthoxanthum odoratum, ApeSpi: Apera spica-venti, ArrEla: Arrhenatherum elatius, BetPub: Betula pubescens, BroHor: Bromus hordeaceus, CalVul: Calluna vulgaris, CarArr: Carex arrenaria, CarSpec 1 \& 2: Carex spp., CerFon: Cerastium fontanum, CerSem: Cerastium semidecandrum, CirArv: Cirsium arvense, ConCan: Conyza canadensis, CreCap: Crepis capillaris, DacGlo: Dactylis glomerata, DesFle: Deschampsia flexuosa, ElyRep: Elytrigia repens, EpiTet: Epilobium tetragonum, EriAnn: Erigeron annuus, EriTet: Erica tetralix, EroVer: Erophila verna, FalCon: Fallopia convolvulus, FesRub: Festuca rubra, FesSpec1: Festuca spec. FilVul: Filago vulgaris, FilMin: Filago minima, FraVes: Fragaria vesca, GalSax: Galium saxatile, GenAng: Genista anglica, GerMol: Geranium molle, GnaSyl: Gnaphalium sylvaticum, HieLae: Hieracium laevigatum, HiePil: Hieracium pilosella, HolLan: Holcus lanatus, HypPer: Hypericum perforatum, HypRad: Hypochaeris radicata, JacVul: Jacobaea vulgaris, JunSqu: Juncus squarossus, JunTen: Juncus tenuis, LeuVul: Leucanthemum vulgare, LeoAut: Leontodon autumnalis, LolPer: Lolium perenne, LotCor: Lotus corniculatus, LuzCam: Luzula campestris, LuzLuz: Luzula luzuloides, MolCar: Molinea caerulea, MyoRas: Myosotis ramosissima, NarStr: Nardus stricta, OriVul: Origanum vulgare, OrnPer: Ornithopus perpusillus, PhlPra: Phleum pratense, PicOmo: Picea omorika, PinNig: Pinus nigra, PinSyl: Pinus sylvestris, PlaLan: Plantago lanceolata, PoaCom: Poa compressa, PoaPra: Poa pratensis, PoaTri: Poa trivialis, PruSer: Prunus serotina, RanRep: Ranunculus repens, RhaFra: Rhamnus frangula, RubFru: Rubus fructuosa, RumAce: Rumex acetosa, RumAcs: Rumex acetosella, SagPro: Sagina procumbens, SclAnn: Scleranthus annuus, ScrNod: Scrophularia nodosa, SenIna: Senecio inaequidens, SolGig: Solidago gigantea, SpeRub: Spergularia rubra, TanVul: Tanacetum vulgare, TarOff: Taraxacum offcinale, TorJap: Torilis japonica, TriArv: Trifolium arvense, TriCam: Trifolium campestre, TriDub: Trifolium dubium, TriRep: Trifolium repens, VerArv: Veronica arvensis, VerOff: Veronica officinalis, VerSer: Veronica serpyllifolia, VicHir: Vicia hirsuta, VicSat: Vicia sativa, VioArv: Viola arvensis.

Table S2.3. Results of MRPP tests for differences in community composition among noninoculated plots (control) and plots that were treated with the two different inocula.

Both analyses with (left) and without (right) the two soil donor sites included in the analysis are shown. n.a. $=$ not available.

\begin{tabular}{|c|c|c|c|c|c|c|}
\hline \multirow[t]{2}{*}{ Response } & \multicolumn{3}{|c|}{ With donor sites (i.e. 5 groups) } & \multicolumn{3}{|c|}{ Without donor sites (i.e. 3 groups) } \\
\hline & Pseudo-F & $\mathrm{p}$-value & $\mathrm{R}^{2}$ & Pseudo-F & p-value & $\mathrm{R}^{2}$ \\
\hline Vegetation & 11.4 & 0.001 & 0.75 & 3.5 & 0.002 & 0.44 \\
\hline $\begin{array}{l}\text { Microbial } \\
\text { community }\end{array}$ & 13.9 & 0.001 & 0.79 & 4.8 & 0.005 & 0.52 \\
\hline Nematodes & 6.6 & 0.001 & 0.64 & 2.1 & 0.006 & 0.32 \\
\hline Microarthropods & n.a. & n.a. & n.a. & 2.6 & 0.02 & 0.36 \\
\hline
\end{tabular}



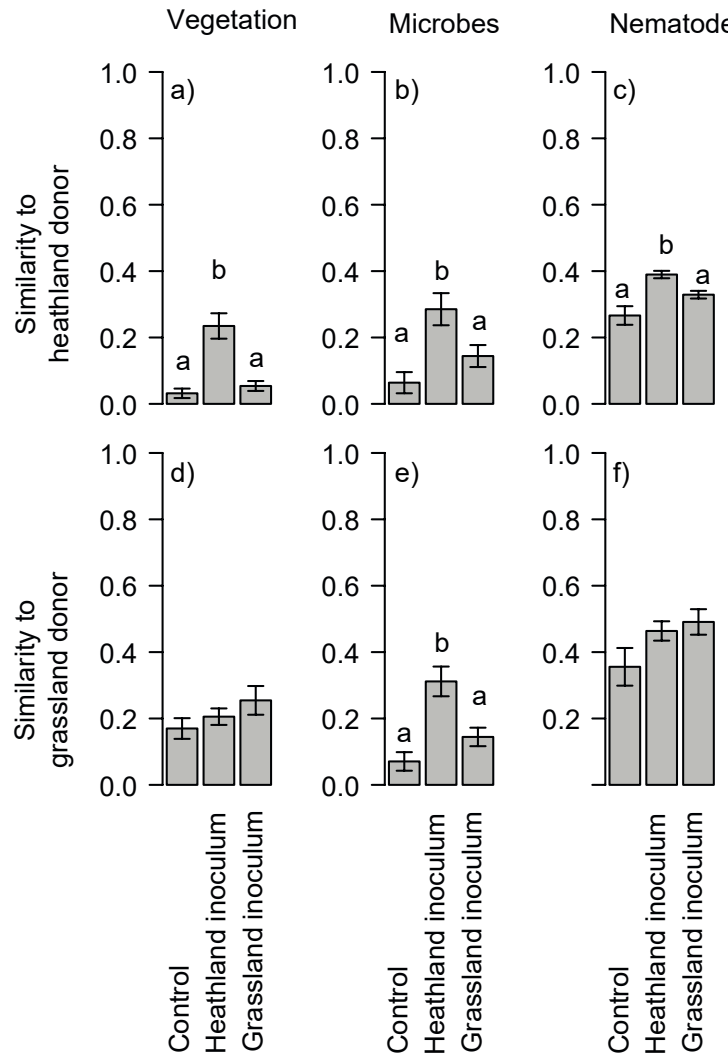

Fig. S2.3. Similarity to donor communities.

Shown is the similarity (mean \pm SE; 1 -Bray-Curtis distance) of the communities in non-inoculated plots (control) and plots that were treated with the two different soil inocula relative to the donor heathland (panels a-c) and the donor grassland (panels $\mathrm{d}-\mathrm{f}$ ) communities respectively. Different letters indicate significant differences among means within panels.

Table S2.4. Statistical analyses for differences in similarity to the donor sites.

Analyses included non-inoculated plots (control) and plots that were treated with the heathland and grassland inoculum respectively (c.f. Fig. S2.3).

\begin{tabular}{lcccc}
\hline Response & \multicolumn{2}{c}{ Heathland donor } & \multicolumn{2}{c}{ Grassland donor } \\
& $\mathrm{F}_{2.6}$ & $\mathrm{p}$-value & $\mathrm{F}_{2.6}$ & p-value \\
\hline Vegetation & 11.1 & 0.01 & 2.7 & 0.15 \\
Microbial community & 6.3 & 0.03 & 8.0 & 0.02 \\
Nematodes & 6.4 & 0.03 & 2.2 & 0.19 \\
\hline
\end{tabular}




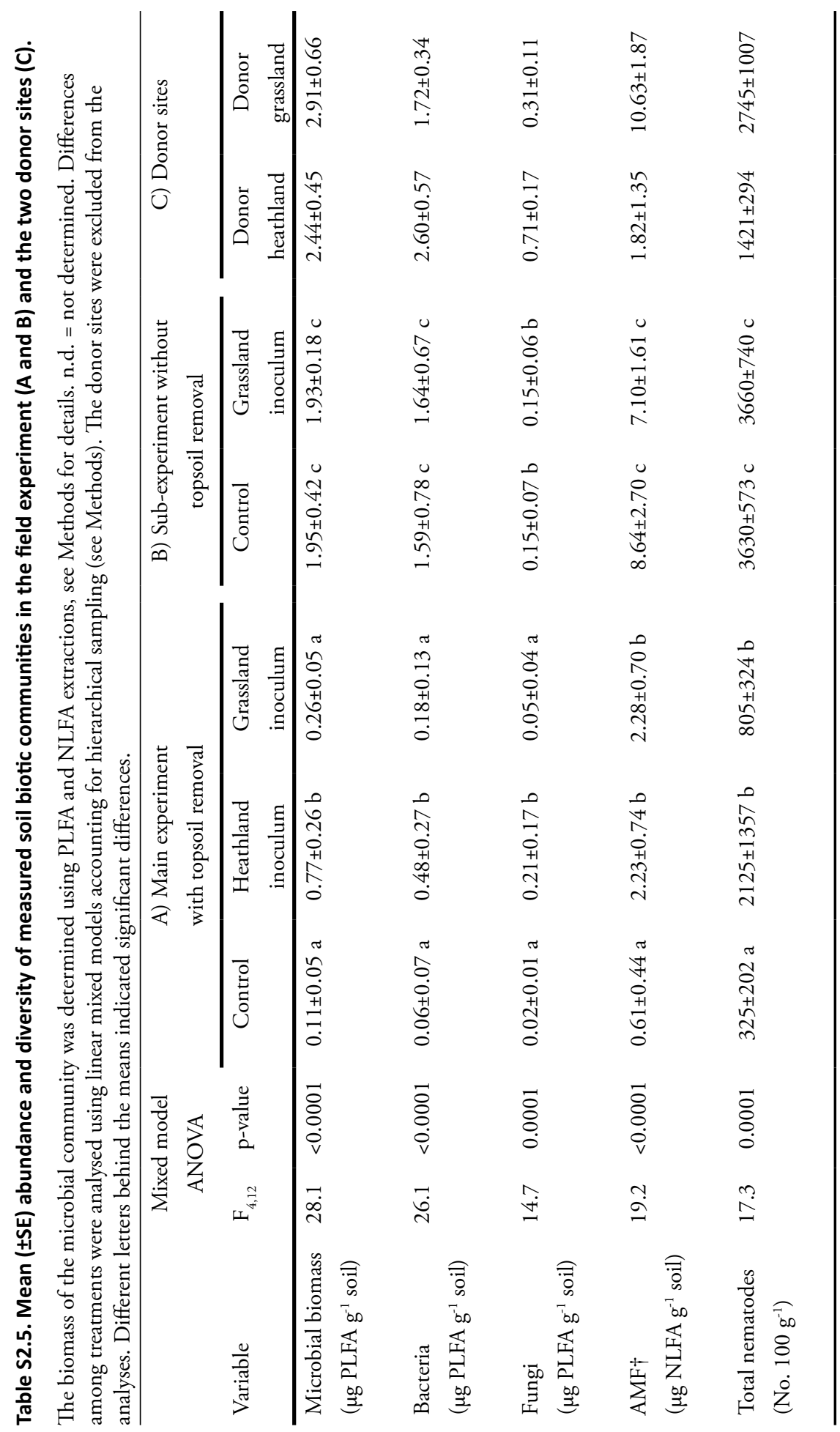




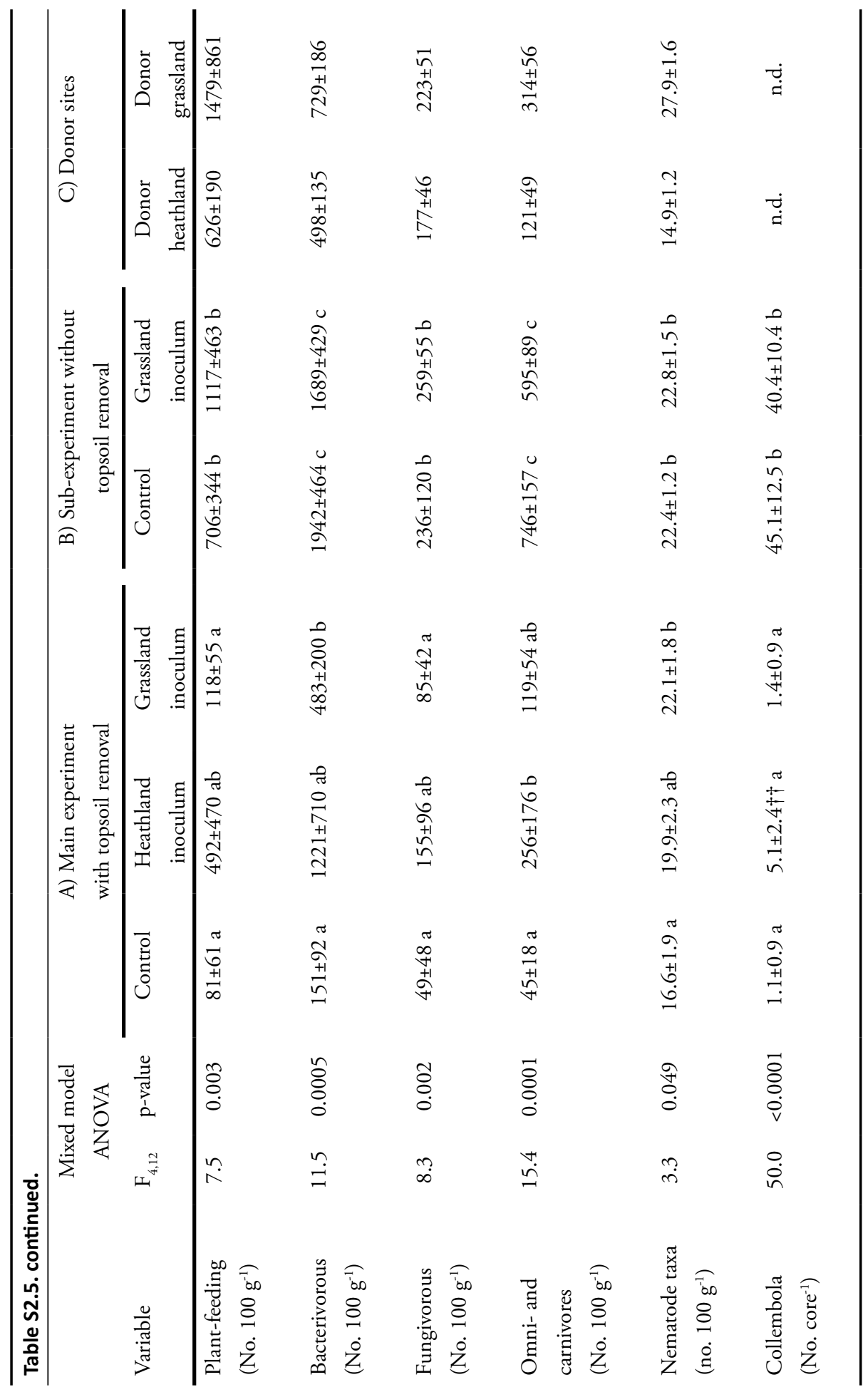




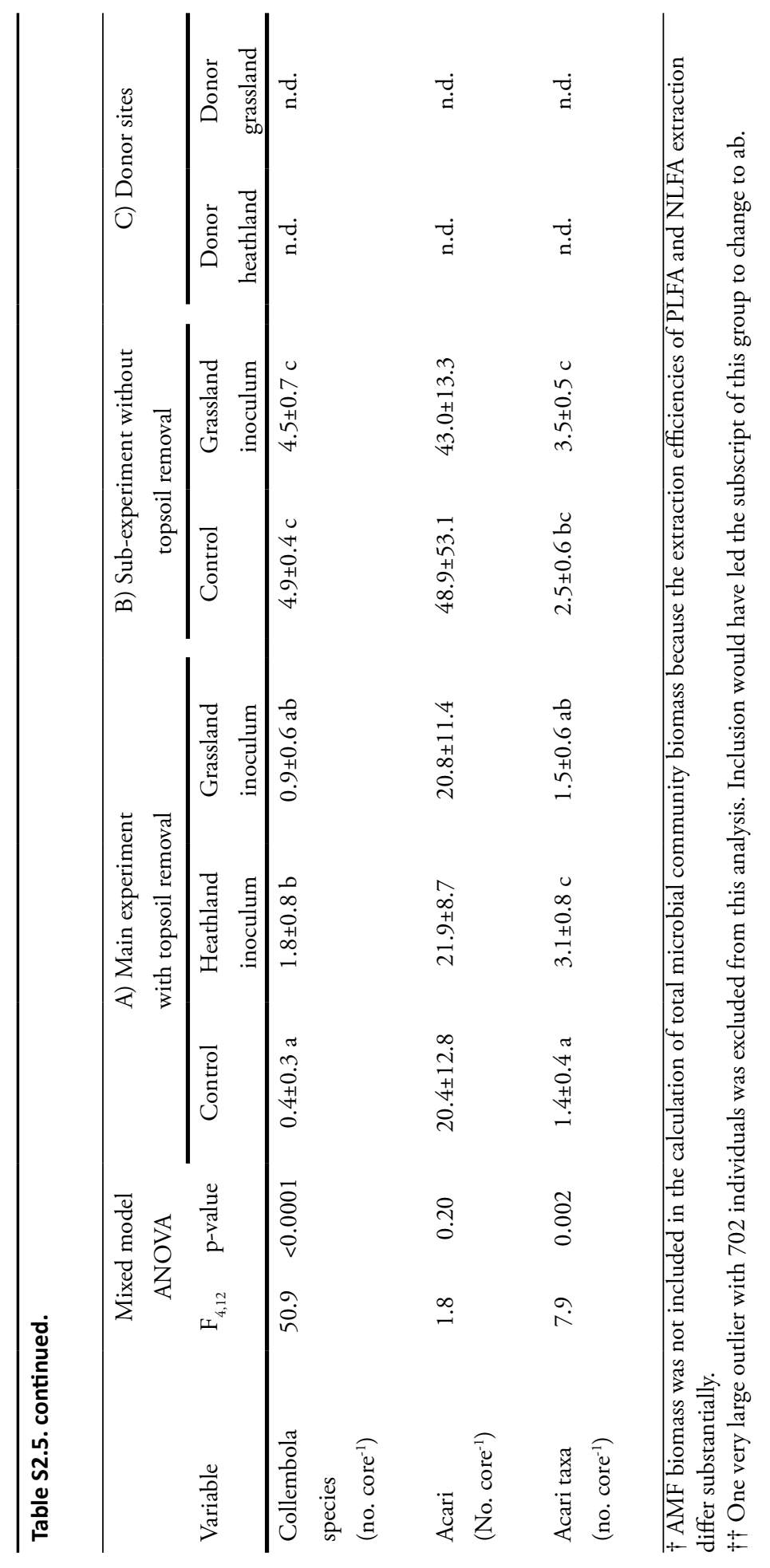




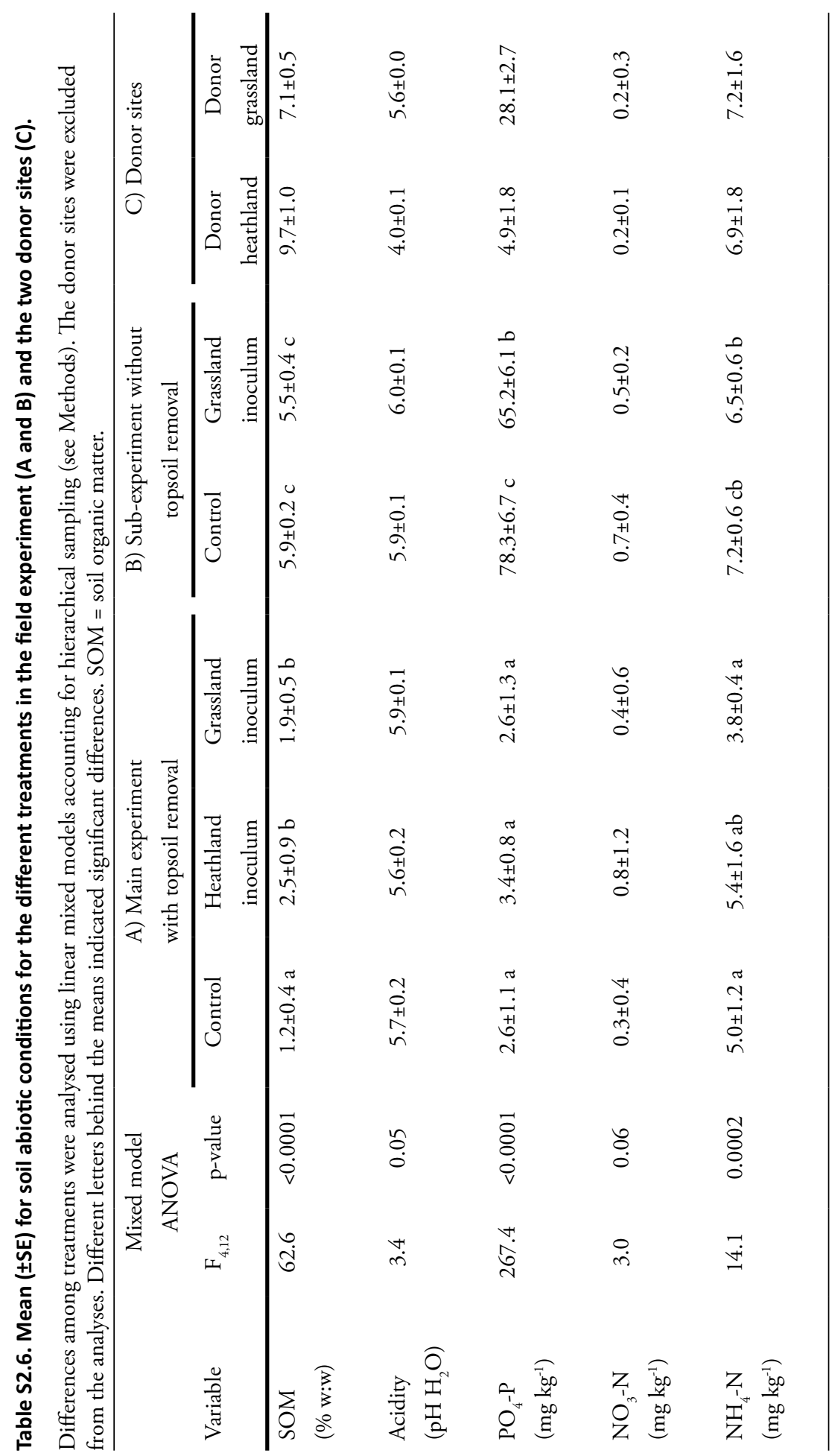


Table S2.7. Statistical analyses for differences in community composition in the mesocosm experiment sown with standardized plant communities of 30 species (Table S2.1).

Differences between inoculated mesocosms and the non-inoculated control ( $\mathrm{H}$ vs. $\mathrm{C}$ and $\mathrm{G}$ vs. C) as well as between the heathland and the grassland inocula (H vs. $\mathrm{G})$ are presented.

\begin{tabular}{|c|c|c|c|c|c|c|}
\hline \multicolumn{2}{|c|}{$\begin{array}{l}\text { a) Test for overall difference } \\
\text { from no effect (i.e. } 0 \text { ) }\end{array}$} & \multicolumn{3}{|c|}{$\begin{array}{l}\text { b) Per treatment tests for difference } \\
\text { from no effect (i.e. 0) }\end{array}$} & \multicolumn{2}{|c|}{$\begin{array}{l}\text { c) Test for among } \\
\text { treatment differences in } \\
\text { effect size }\end{array}$} \\
\hline $\mathrm{F}_{3,9}$ & $\mathrm{p}$ & Treatment & $t_{9}$ & $\mathrm{p}$ & $\mathrm{F}_{2,9}$ & $\mathrm{p}$ \\
\hline \multirow[t]{3}{*}{168.7} & $<0.0001$ & $\mathrm{H}$ vs $\mathrm{C}$ & 13.2 & $<0.0001$ & 2.28 & 0.16 \\
\hline & & G vc C & 14.2 & $<0.0001$ & & \\
\hline & & $\mathrm{H}$ vs $\mathrm{G}$ & 11.4 & $<0.0001$ & & \\
\hline
\end{tabular}

Table S2.8. Statistical analyses for differences from the control in plots with and without topsoil removal.

$\mathrm{C}=$ control, $\mathrm{G}=$ grassland-inoculated, $\mathrm{H}=$ heathland-inoculated, rem = topsoil removed, intact = topsoil was left intact.

\begin{tabular}{|c|c|c|c|c|c|c|c|}
\hline \multirow[t]{2}{*}{ Response } & \multicolumn{2}{|c|}{$\begin{array}{l}\text { a) Test for overall } \\
\text { difference from no } \\
\text { effect (i.e. 0) }\end{array}$} & \multicolumn{3}{|c|}{$\begin{array}{l}\text { b) Per treatment tests for difference } \\
\text { from no effect (i.e. 0) }\end{array}$} & \multicolumn{2}{|c|}{$\begin{array}{l}\text { c) Test for } \\
\text { among } \\
\text { treatment } \\
\text { differences }\end{array}$} \\
\hline & $\mathrm{F}_{3.6}$ & $\mathrm{p}$ & Treatment & $\mathrm{t}_{6}$ & $\mathrm{p}$ & $\mathrm{F}_{2,6}$ & $\mathrm{p}$ \\
\hline \multirow[t]{3}{*}{ Vegetation } & \multirow[t]{3}{*}{77.1} & \multirow[t]{3}{*}{$<0.0001$} & $\mathrm{H}_{\text {rem }}$ vs. $\mathrm{C}_{\text {rem }}$ & 13.9 & $<0.0001$ & \multirow[t]{3}{*}{5.8} & \multirow[t]{3}{*}{0.04} \\
\hline & & & $\mathrm{G}_{\mathrm{rem}}$ vs. $\mathrm{C}_{\mathrm{rem}}$ & 12.8 & $<0.0001$ & & \\
\hline & & & $G_{\text {intact }}$ vs. $C_{\text {intact }}$ & 13.6 & $<0.0001$ & & \\
\hline \multirow{3}{*}{$\begin{array}{l}\text { Microbial } \\
\text { community }\end{array}$} & \multirow[t]{3}{*}{96.8} & \multirow[t]{3}{*}{$<0.0001$} & $\mathrm{H}_{\mathrm{rem}}$ vs. $\mathrm{C}_{\mathrm{rem}}$ & 15.8 & $<0.0001$ & \multirow[t]{3}{*}{13.2} & \multirow[t]{3}{*}{0.006} \\
\hline & & & $\mathrm{G}_{\mathrm{rem}}$ vs. $\mathrm{C}_{\mathrm{rem}}$ & 10.8 & $<0.0001$ & & \\
\hline & & & $\mathrm{G}_{\text {intact }}$ vs. $\mathrm{C}_{\text {intact }}$ & 9.9 & 0.0001 & & \\
\hline \multirow[t]{3}{*}{ Nematodes } & \multirow[t]{3}{*}{132.6} & \multirow[t]{3}{*}{$<0.0001$} & $\mathrm{H}_{\mathrm{rem}}$ vs. $\mathrm{C}_{\mathrm{rem}}$ & 8.8 & 0.0001 & \multirow[t]{3}{*}{7.0} & \multirow[t]{3}{*}{0.03} \\
\hline & & & $\mathrm{G}_{\mathrm{rem}}$ vs. $\mathrm{C}_{\mathrm{rem}}$ & 11.1 & $<0.0001$ & & \\
\hline & & & $G_{\text {intact }}$ vs. $C_{\text {intact }}$ & 14.1 & $<0.0001$ & & \\
\hline \multirow{3}{*}{$\begin{array}{l}\text { Micro- } \\
\text { arthropods }\end{array}$} & \multirow[t]{3}{*}{50.5} & \multirow[t]{3}{*}{0.0001} & $\mathrm{H}_{\mathrm{rem}}$ vs. $\mathrm{C}_{\mathrm{rem}}$ & 6.4 & 0.0007 & \multirow[t]{3}{*}{8.7} & \multirow[t]{3}{*}{0.02} \\
\hline & & & $\mathrm{G}_{\mathrm{rem}}$ vs. $\mathrm{C}_{\mathrm{rem}}$ & 7.2 & 0.0004 & & \\
\hline & & & $G_{\text {intact }}$ vs. $C_{\text {intact }}$ & 7.7 & 0.0002 & & \\
\hline
\end{tabular}




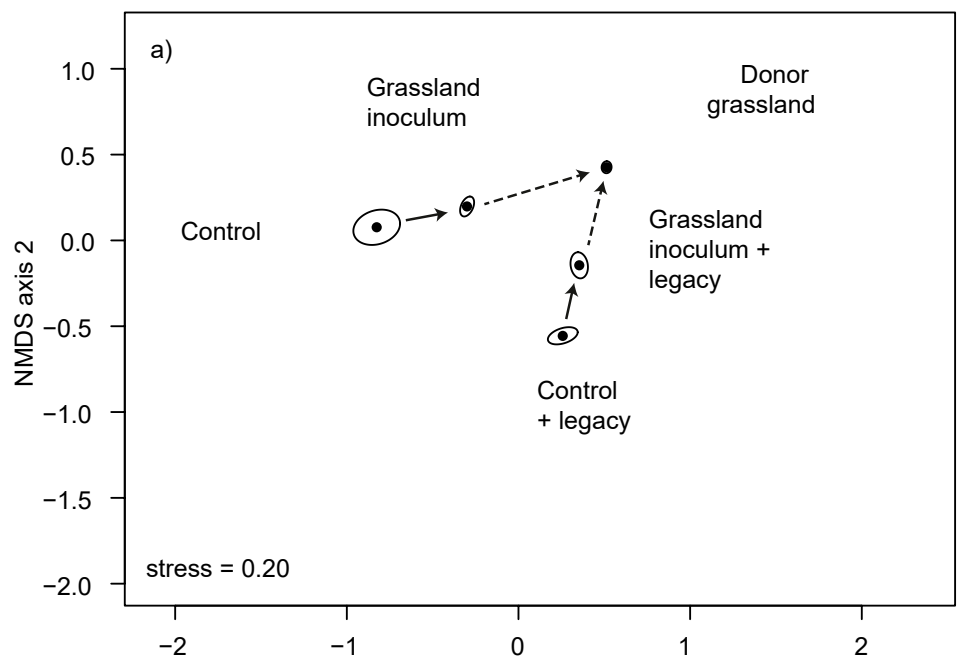

NMDS axis 1

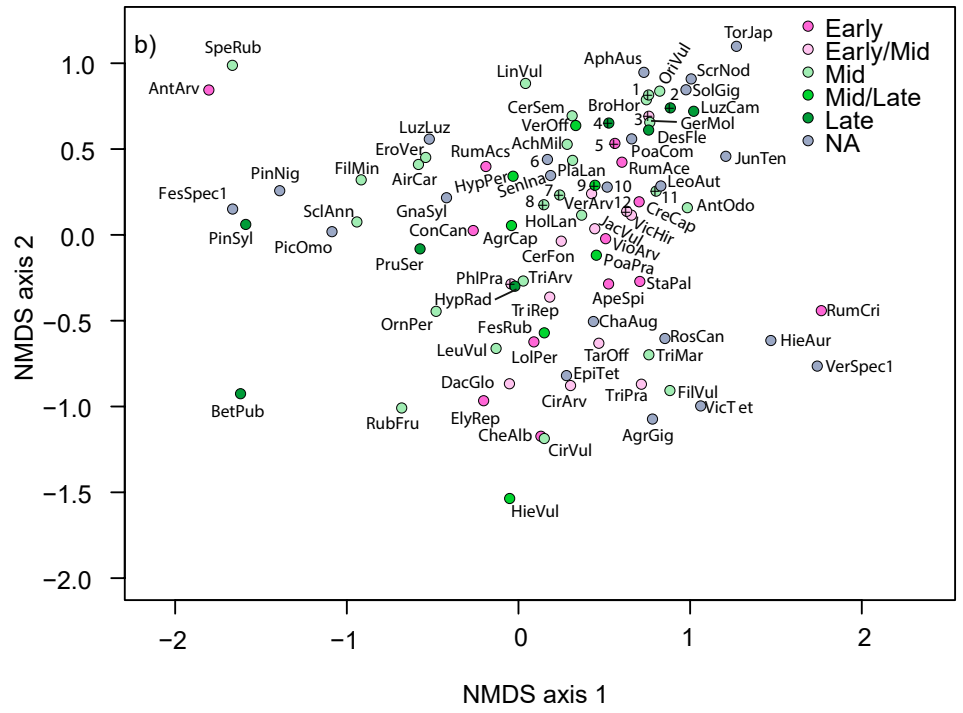

Fig. S2.4. Plant species composition in field experiment in treatments with or without topsoil removal and soil inoculation.

The top panel (a) show the means (dots) and SEs (ellipses) of the treatments as well as the donor site as visualized using NMDS (MRPP Pseudo F $=4.77, \mathrm{p}=0.001$ ). The bottom panel (b) shows the species scores on the same NMDS plot. Some species names could not be plotted and are displayed with a numbered plus sign (see below). Species abbreviations are: AphAus: Aphanes australis, AgrCap: Agrostis capillaris, AgrGig: Agrostis gigantea, AirCar: Aira caryophyllea, AntArv: Anthemis arvensis, AntOdo: Anthoxanthum odoratum, ApeSpi: Apera spica-venti, BetPub: Betula 


\section{Fig. S2.4. caption continued.}

pubescens, BroHor: Bromus hordeaceus, CerFon: Cerastium fontanum, CerSem: Cerastium semidecandrum, ChaAug: Chamerion augustifolium, CheAlb: Chenopodium album, CirArv: Cirsium arvense, CirVul: Cirsium vulgare, ConCan: Conyza Canadensis, CreCap: Crepis capillaris, DacGlo: Dactylis glomerata, DesFle: Deschampsia flexuosa, ElyRep: Elytrigia repens, EpiTet: Epilobium tetragonum, EroVer: Erophila verna, FesRub: Festuca rubra, FesSpec1: Festuca spec., FilVul: Filago vulgaris, FilMin: Filago minima, GerMol: Geranium molle, GnaSyl: Gnaphalium sylvaticum, HieAur: Hieracium aurantiacum, HieLae: Hieracium laevigatum, HieVul: Hieracium vulgatum, HolLan: Holcus lanatus, HypPer: Hypericum perforatum, HypRad: Hypochaeris radicata, JacVul: Jacobaea vulgaris, JunTen: Juncus tenuis, LeuVul: Leucanthemum vulgare, LeoAut: Leontodon autumnalis, LinVul: Linaria vulgaris, LolPer: Lolium perenne, LotCor: Lotus corniculatus, LuzCam: Luzula campestris, LuzLuz: Luzula luzuloides, NarStr: Nardus stricta, OriVul: Origanum vulgare, OrnPer: Ornithopus perpusillus, PhlPra: Phleum pratense, PicOmo: Picea omorika, PinNig: Pinus nigra, PinSyl: Pinus sylvestris, PlaLan: Plantago lanceolata, PoaCom: Poa compressa, PoaPra: Poa pratensis, PoaTri: Poa trivialis, PruSer: Prunus serotina, RosCan: Rosa canina, RubFru: Rubus fructuosa, RumAce: Rumex acetosa, RumAcs: Rumex acetosella, RumCri: Rumex crispus, SclAnn: Scleranthus annuus, ScrNod: Scrophularia nodosa, SenIna: Senecio inaequidens, SolGig: Solidago gigantea, SpeRub: Spergularia rubra, StaPal: Stachys palustris, TanVul: Tanacetum vulgare, TarOff: Taraxacum offcinale, TorJap: Torilis japonica, TriArv: Trifolium arvense, TriMar: Tripleurospermum maritimum, TriPra: Trifolium pratense, TriRep: Trifolium repens, VerArv: Veronica arvensis, VerOff: Veronica officinalis, VerSpec1: Verbascum spec., VicHir: Vicia hirsuta, VicSat: Vicia sativa, VicTet: Vicia tetrasperma, VioArv: Viola arvensis, 1: Arrhenatherum elatius, 2: Calluna vulgaris, 3: Ranunculus repens, 4: Hieracium pilosella, 5: Fallopia convolvulus, 6: Carex spec. 2, 7: Trifolium dubium, 8: Trifolium campestre, 9: Sagina procumbens, 10: Veronica serpyllifolia, 11: Myosotis ramosissima, 12: Vicia sativa.

Table S2.9. Results of statistical analysis of soil inoculation effects in the field and mesocosm experiments on species in the different successional groups.

For membership of the species to the four groups see the caption of Fig. S2.

\begin{tabular}{llccc}
\hline Experiment & Response & d.f. & F-value & p-value \\
\hline Field & Grass target spp & 2,6 & 9.71 & 0.01 \\
& Heath target spp & 2,6 & 17.48 & 0.003 \\
& Early spp & 2,6 & 0.54 & 0.61 \\
\multirow{3}{*}{ Mesocosm } & Mid spp & 2,6 & 2.90 & 0.13 \\
& Grass target spp & 2,6 & 31.48 & $<0.001$ \\
& Heath target spp & 2,6 & 5.46 & 0.045 \\
& Early spp & 2,6 & 1.84 & 0.24 \\
& Mid spp & 2,6 & 4.19 & 0.07 \\
\hline
\end{tabular}




\section{$(w y)$}

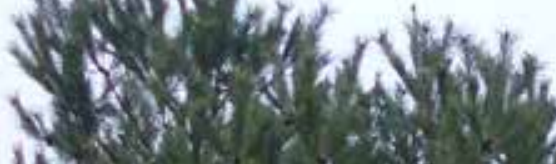

(4.)

(180.

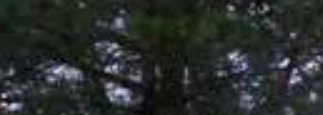

souds

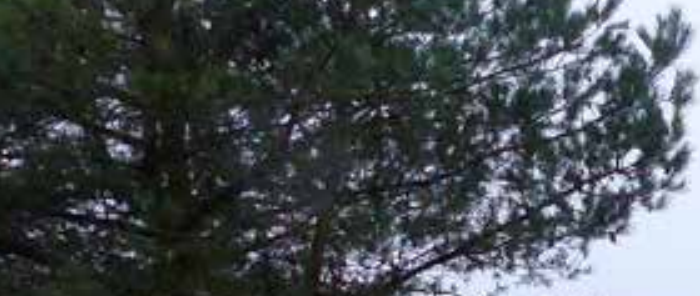

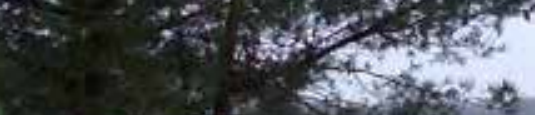

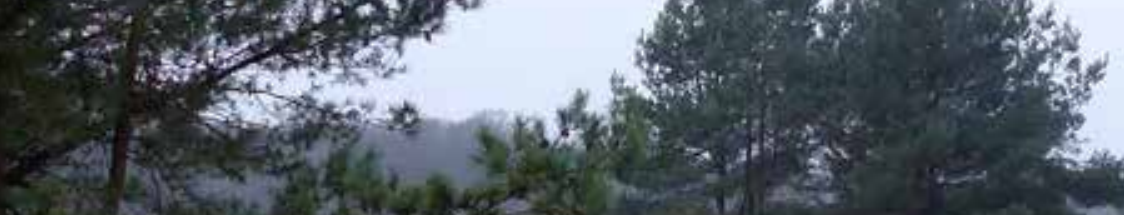

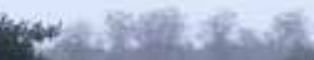

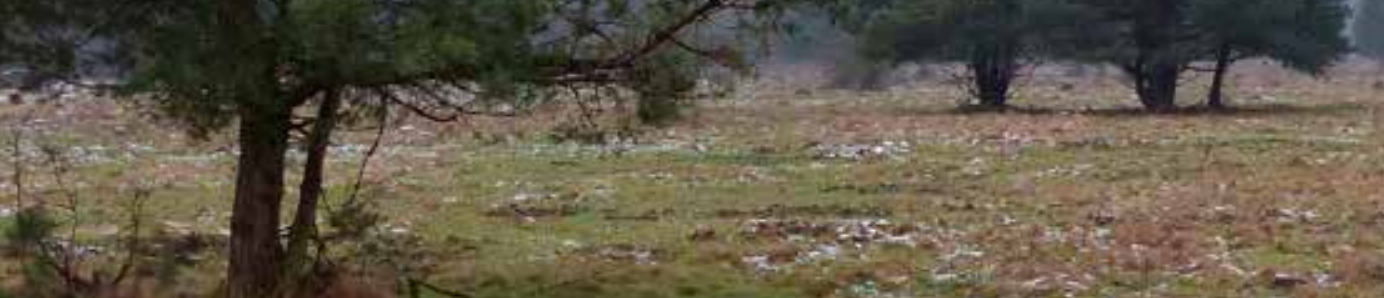

is

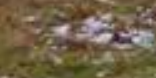

50 t:

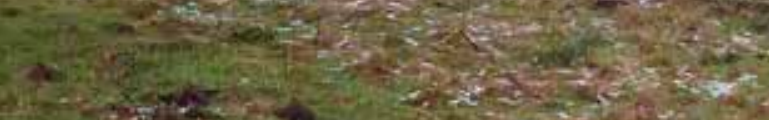

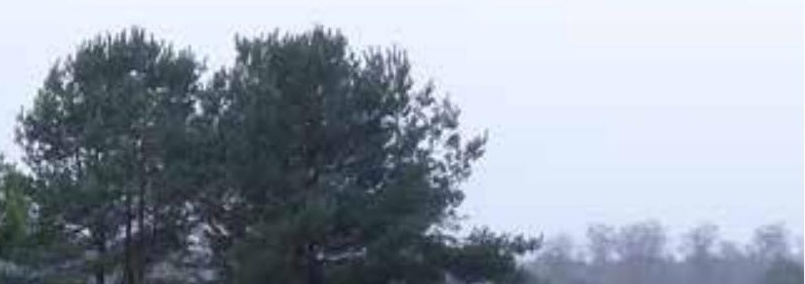

1.

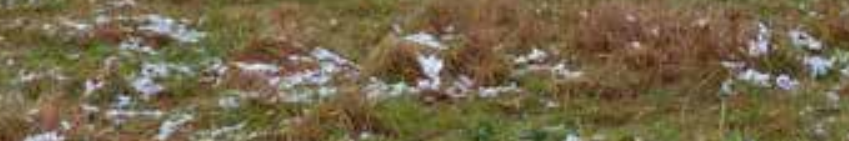

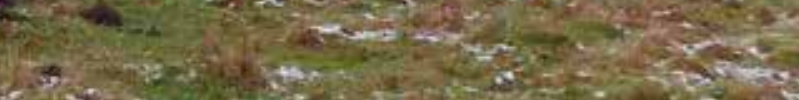

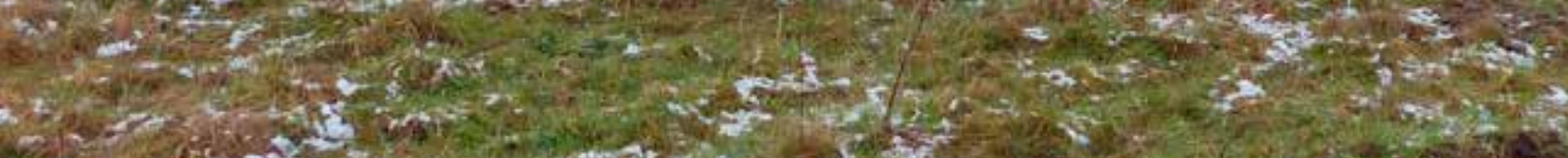

2.

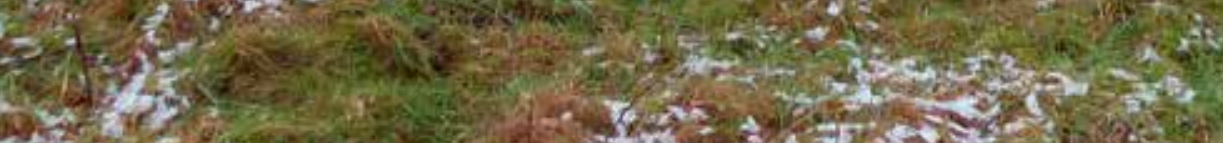

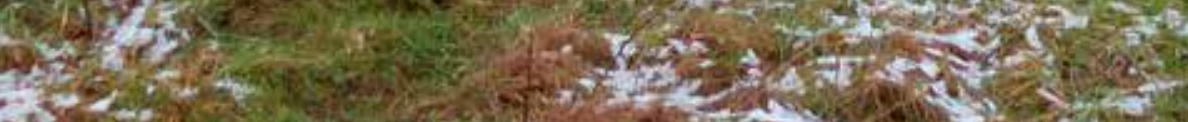

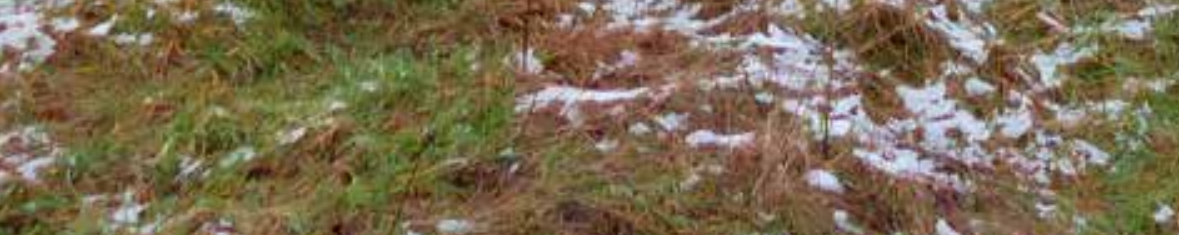

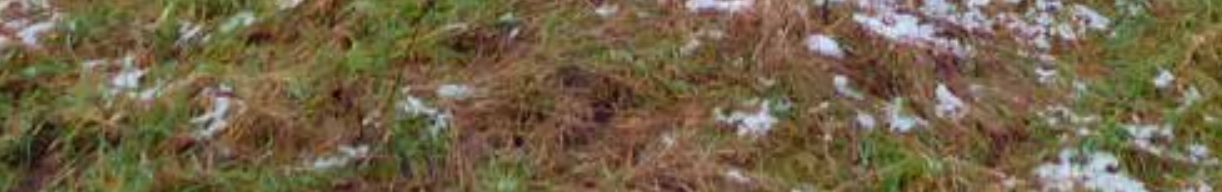


CHAPTER 3

Potential for synergy in soil inoculation for nature restoration by mixing inocula from different successional stages

E. R. Jasper Wubs, Pauline D. Melchers, and T. Martijn Bezemer 


\begin{abstract}
Soil inoculation has been shown to be a powerful tool for the restoration of terrestrial ecosystems. However, the origin of the donor material may differentially influence early- and late-successional plant species. For the successful restoration of speciesrich late-successional plant communities, early-successional ruderal species need to be suppressed and late-successional target species promoted. Donor soil from late-succession stages may benefit target plant species due to a higher abundance of soil-borne mutualists. Arable soils, on the other hand, may suppress ruderals as they support more root herbivores that preferentially attack ruderal plant species, while mid-succession soils may be intermediate. We hypothesized that a mixture of arable and late-succession inocula may outperform pure late-successional inocula.
\end{abstract}

We conducted a greenhouse experiment and tested the growth of ruderal and target (late-successional) plant species on pure and mixed inocula. The inocula were derived from arable fields, mid-succession grasslands and late-succession heathlands occurring on the same soil type and we created a replacement series for each of these inocula types.

In general, we found that a higher proportion of heathland material led to a higher biomass of target plant species, while responses of ruderal species were variable. We found synergistic effects when inocula were mixed. In particular, a 50:50 mixture of heathland and arable soil in the inoculum led to a significant reduction in ruderal species biomass relative to the two respective pure inocula.

We conclude that mixing inocula from different successional stages may lead to synergistic effects on restoration, but this depends highly on the specific combination of inocula that is used. This suggest that specific inocula may need to be developed in order to rapidly restore different plant communities.

\title{
Introduction
}

Many natural ecosystems need to be restored in order to reach international conservation targets (Vitousek et al. 1997, Hobbs and Harris 2001). Plant and soil communities tightly interact and plant-soil interactions are thought to play a key role during ecological restoration (Reynolds et al. 2003, Eviner and Hawkes 2008, Kardol and Wardle 2010). Soil inoculation, where whole (late-successional) soil communities are introduced to areas to be restored can be a powerful tool to rapidly restore terrestrial ecosystems (Harris 2009, Chapter 2). Using a large field experiment in an area where nature is restored on former arable land, we recently 
showed that application of whole soil inocula sourced from a target grassland or heathland steered the development of the ecosystem under restoration in the direction of its respective donor (Chapter 2). How the composition of soil inocula can be optimized and whether potential synergies among inoculum sources exist is unclear.

The net effect of the soil community on plants is determined by the balance of the actions of plant antagonists, symbionts and decomposers (Van der Putten et al. 2016). Antagonists include soil-borne pathogens and root herbivores, while symbionts include mycorrhizal fungi and plant-growth promoting rhizobacteria. The composition and abundance of the soil community changes considerably during natural succession (Kardol et al. 2005, Van der Wal et al. 2006, Bauer et al. 2015, Frouz et al. 2016). For example, the abundance of nematode and insect root herbivores is initially high on arable fields and then declines during secondary succession (Brown and Gange 1992, Korthals et al. 2001, Verschoor et al. 2001, Kardol et al. 2005, Rasmann et al. 2011). Tillage in arable cultivation reduces the abundance and diversity of many soil taxa (Tsiafouli et al. 2015), including myccorhizal fungi (Helgason et al. 1998). Subsequently, during secondary succession on former arable land the abundance of mycorrhiza increases (Janos 1980, Johnson et al. 1991, Barni and Siniscalco 2000, Castle et al. 2016) and their composition changes (Johnson et al. 1991, Barni and Siniscalco 2000). These patterns in soil community development during secondary succession may be used to optimize soil inocula.

For the successful restoration of species-rich late-successional communities, earlysuccessional ruderal species need to be suppressed and late-successional target species promoted. Early-successional plants tend to be sensitive to antagonists, while late-successional species, typically the target species for conservation and restoration, are more dependent on soil-borne symbionts (Reynolds et al. 2003, Kardol et al. 2007, 2013, Middleton and Bever 2012). For instance, root herbivores are known to feed selectively on early-successional ruderal plant species due to their higher palatability and low investment in defence (Brown and Gange 1992, Fraser and Grime 1999). Late-successional plants respond most strongly to inoculation with mycorrhizal fungi (Middleton and Bever 2012), particularly when locally sourced material is used (Middleton et al. 2015). In addition, it has been shown, both in the greenhouse and in the field, that inoculation with a late-successional soil community can differentially affect the performance of ruderal and target species and that late-successional soil inocula can promote restoration success 
(De Deyn et al. 2003, Kardol et al. 2006, Carbajo et al. 2011, Middleton and Bever 2012, Chapter 2). However, even though the growth of late-successional plants was improved, they did not gain dominance over the early-successional plants in these experiments. To maximize the effectiveness of soil inoculation, the inoculum should both suppress ruderals and promote late-successional species. We hypothesise this can be achieved by mixing inocula from both early- and latesuccessional origin. To our knowledge this has not been tested.

In this study we tested how different mixtures of soil inocula affect the performance of ruderal and target plant species (Fig. 3.1). We tested mixtures of soils sampled from arable fields, mid-succession grasslands and late-succession heathlands, with a replacement series among each pair of inoculum sources. We expected that inocula with a high proportion of material from heathlands would promote the performance of the target plant species and that inocula with a high proportion

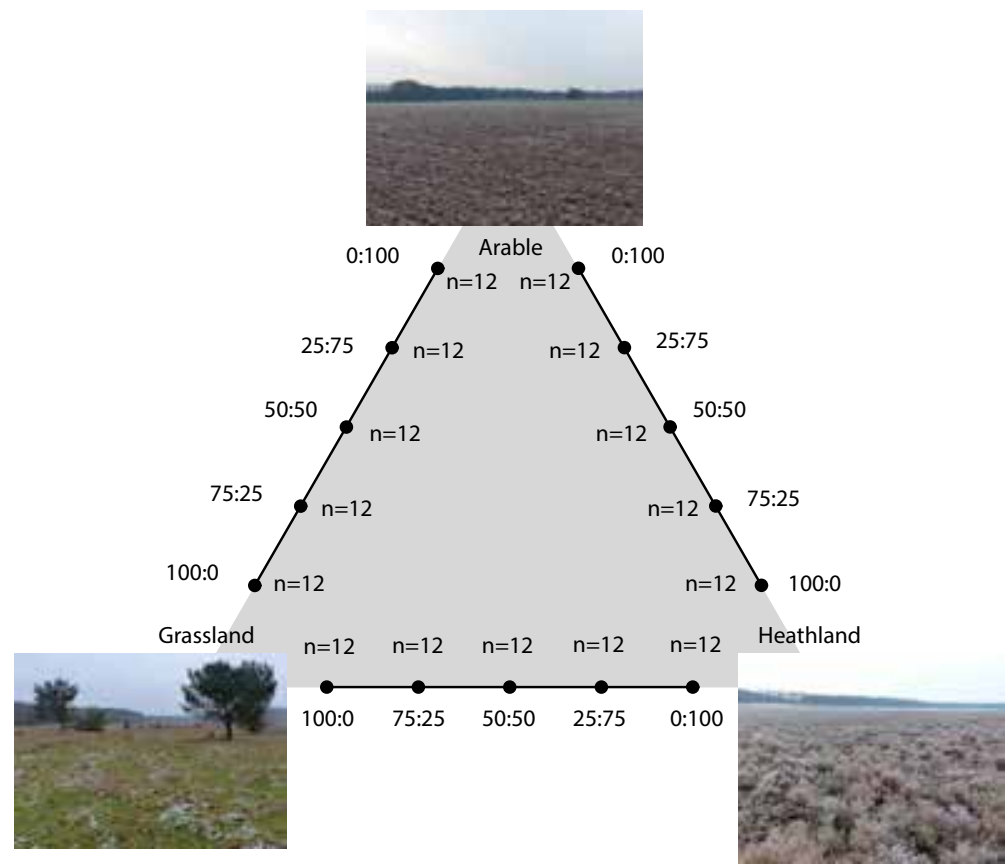

Fig. 3.1. Experimental design where inocula of arable fields, mid-succession grasslands, and dry heathlands were mixed in replacement series.

The photos show one field of each type at the time of sampling. This design was replicated over three sets of fields (field triplets; Table S3.1). The number of replicates per inoculum type and replacement series is indicated. 
of arable field soil would suppress ruderals. Mid-succession grassland soils were expected to have intermediate effects on target and ruderal species as they still contain relatively high amounts of soil-borne antagonists, but also mutualists. Furthermore, we expected that a mixture of early and heathland inoculum would lead to the best and worst performance of target and ruderal species respectively, because both plant symbionts and antagonists would be present in the mixed inoculum. We explicitly tested for synergy among inoculum sources by comparing plant performance on mixed inocula to performance on the pure inocula.

\section{Methods}

We conducted a greenhouse experiment where a common background soil was inoculated with different inocula (9:1 w:w). Both the inocula and the background soil were collected in January 2015 on sandy or sandy loam glacial deposits in the central part of the Netherlands (Table S3.1). The background soil was collected from the Reijerscamp, an ex-arable field of 160 ha that has been undergoing restoration since 2006. The field has been in intensive agricultural use at least since World War 2 until 2004. Then, it was used for extensive wheat cultivation for two years prior to the implementation of extensive restoration measures (Chapter 2). The soil was collected from the central part of the field, where the only management consisted of cattle grazing (25-30 cows throughout the year) and removal of tree seedlings (particularly Betula spp. and Prunus serotina). Approximately $1300 \mathrm{~kg}$ of soil was collected from the organic soil layer within $10-50 \mathrm{~cm}$ depth and subsequently sieved over a $1 \mathrm{~cm}$ mesh to remove major roots and stones and homogenized. The background soil was sterilized (>25KGray gamma radiation, Isotron, Ede, the Netherlands) to eliminate the resident soil community.

Three types of inocula were sampled from three fields each within the study region (Table S3.1). The types were: 1 ) arable fields (cereals in recent years) in extensive organic cultivation with annual tillage (arable, A), 2) ex-arable grasslands that had undergone 27-33 yrs of secondary succession (grassland, G), and 3) dry heathlands that have been in existence at least since the $13^{\text {th }}$ century (heathland, $\mathrm{H})$. We divided the nine fields into three groups of three (field triplet), each group containing one field of each type, based on geographic proximity. The distance between any pair of fields used for mixing inocula was between 0.7 and $5.7 \mathrm{~km}$. Within each field an area of $5 \times 5 \mathrm{~m}$ was selected at least $20 \mathrm{~m}$ from the edge of the field. At each corner of the selected area $5 \mathrm{~kg}$ of soil was collected from the upper $10-15 \mathrm{~cm}$. The soil was sieved over a $1 \mathrm{~cm}$ mesh to remove stones and large roots. Upon return to the lab the four samples per field were pooled based on 
equal amounts of dry weight resulting in homogenized inoculum material of 20 $\mathrm{kg}$ per field. Three subsamples were taken per pooled inoculum and analysed for chemical composition (Table S3.1). The subsamples were oven-dried for five days at $40{ }^{\circ} \mathrm{C}$ and analysed for soil organic matter content $\left(24 \mathrm{~h}, 430{ }^{\circ} \mathrm{C}\right.$ ), acidity (in $\left.\mathrm{H}_{2} \mathrm{O}\right), \mathrm{N}$ (KCl-extraction) and P (Olsen's extraction) content.

Within each field triplet, inocula of each of the three types (arable, grassland and heathland) were mixed in a replacement series. Mixtures were made for each pair of fields within the field triplet based on dry weight in five ratios: 100:0, 75:25, 50:50, 25:75, 0:100 (Fig. 3.1). For each replacement series a separate set of pure inocula replicates was used. Each treatment was replicated four times ( 3 triplets $\mathrm{x}$ 3 replacement series per triplet $\mathrm{x} 5$ inocula mixing levels per series $\mathrm{x} 4$ replicates $=$ 180 experimental units).

The experiment was conducted in pots $(17 \times 17 \times 17 \mathrm{~cm})$ which were filled with homogenized soil containing $3.6 \mathrm{~kg}$ of background soil and $400 \mathrm{~g}$ inoculum $(9: 1 \mathrm{w}: \mathrm{w} \mathrm{dw})$. We used six plant species as a test community. Three species were ruderals: Crepis capillaris (L.) Wallr. (Asteraceae), Lolium perenne L. (Poaceae) and Myosotis arvensis (L.) Hill (Boraginaceae), and three were conservation target species: Arnica montana L. (Asteraceae), Festuca filiformis Pourr. (Poaceae) and Campanula rotundifolia L. (Campanulaceae), with one grass and two forbs in each group. Seeds were obtained from commercial suppliers of wild plant seeds (Cruydthoeck, Assen, the Netherlands and B\&T World Seeds, Paguignan, France) and germinated (sterilized $1 \mathrm{~min}$. in $5 \% \mathrm{NaClO}$ solution) on moistened glass beads in a climate chamber ( $12 \mathrm{~h}$ light/dark cycle, $20{ }^{\circ} \mathrm{C}$ by day and $15{ }^{\circ} \mathrm{C}$ at night). Two individuals of each species were planted in a random position in a circle in the soil of each pot. Any seedlings that died during the first two weeks were replaced. The pots were placed in the greenhouse in a randomized block design where the blocks corresponded to the field triplets (i.e. three blocks). The plants were allowed to grow in the glasshouse (16:8 h day: night, natural light supplemented with 600 W metal-halide lamps, 1 per $4 \mathrm{~m}^{-2}$, approx. $225 \mu$ mol light quanta $\mathrm{m}^{-2} \mathrm{~s}^{-1}$ at plant level, $21: 16^{\circ} \mathrm{C}$ day: night, $50-70 \%$ relative humidity) for 7 weeks and watered three times per week. Subsequently the shoots of each species were clipped at the soil surface, oven-dried for two days at $75^{\circ} \mathrm{C}$ and weighed.

\section{Data analysis}

We analysed univariate response data using linear mixed models (LMMs), with separate models for total biomass, ruderal biomass and target biomass and a 
separate model for each of the six plant species. We included a random effect for block in the analysis and a fixed effect for inoculum treatment. In addition, we tested how the plant species responded to each kind of inoculum material. To do so we conducted separate regression analyses, with the same random effects structure as above, for each replacement series (i.e. arable-grassland, arable-heathland, and grassland-heathland). Here the proportion of one of the two inoculum sources in the mixture was used as a continuous predictor (e.g. proportion arable, i.e. 0 , 25, 50, 75 and 100, for arable-grassland mixture). We used a multiple response permutation procedure (MRPP) to test for overall differences in plant community composition and permutation was restricted to permutation within blocks.

We tested for synergy in the mixed inocula by calculating the expected performance of the ruderal and target plant species groups based on the pure inocula and the ratios in which their material was present in the mixture. We then subtracted the expected performance from the actual observed performance in the pots with mixed inocula. The expected performance was calculated separately for each pair of fields. Differences between observed and expected performance were analysed in LMMs with a random effect for blocks and mixed inoculum treatment (nine levels) as a fixed effect. Within these models significant deviations from zero (i.e. no synergistic effect) were tested as planned contrasts (Adbi and Williams 2010).

All analyses were conducted in R 3.3.1 (R Core Team 2016), LMMs were modelled using nlme 3.1-128 (Pinheiro et al. 2016), MRPP using vegan 2.4-1 (Oksanen et al. 2016). Model assumptions were checked graphically and heteroscedasticity was modelled using generalized least squares (Pinheiro and Bates 2000, Zuur et al. 2009). Post-hoc test were performed using the lsmeans 2.23-5 package (Lenth 2015) using the false discovery rate to correct for multiple comparisons (Benjamini and Hochberg 1995).

\section{Results}

In general, the composition of the soil inoculum influenced plant community composition (Fig. 3.2; MRPP Pseudo-F $=4.07, \mathrm{p}=0.001$ ). The biomass of the target species increased by $33 \%$ with an increasing fraction of heathland material in the inoculum (Fig. 3.2a; Table 3.1). Biomass of the ruderal species was lower in the A50-H50, G100 (A-G series) and H100 (G-H series) inoculum treatments compared to the other treatments (Fig. 3.2c; Table 3.1). The same pattern was found for total plant biomass (Fig. S3.1; Table 3.1). 
a)

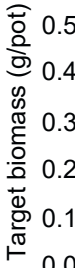

b)

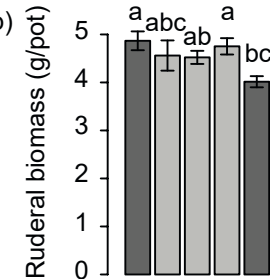

요용

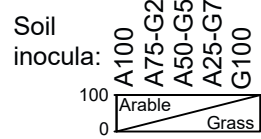

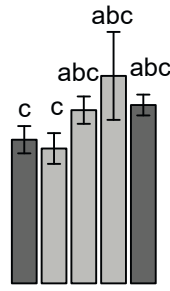

abcabc $a_{b c} a b c$

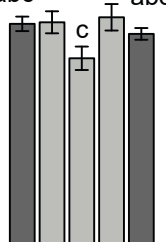

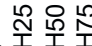

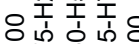

눈 윤 은

Arable Heath

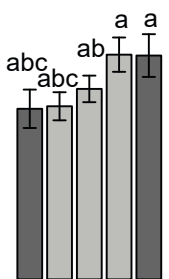

a $a b a b a b$

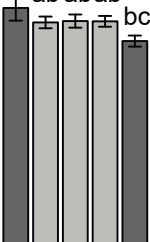

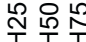

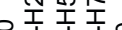

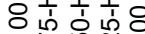

৫ั

c)

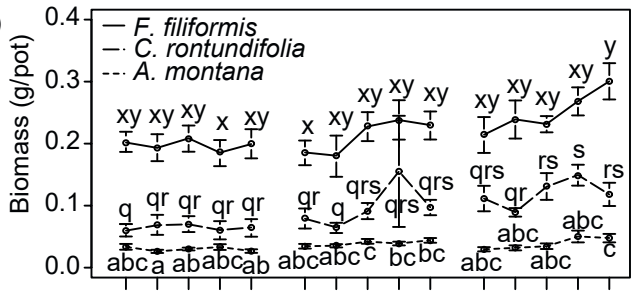

d) $3.0-\begin{aligned} & - \text { C. capillaris } \\ & - \text { L. perenne }\end{aligned}$
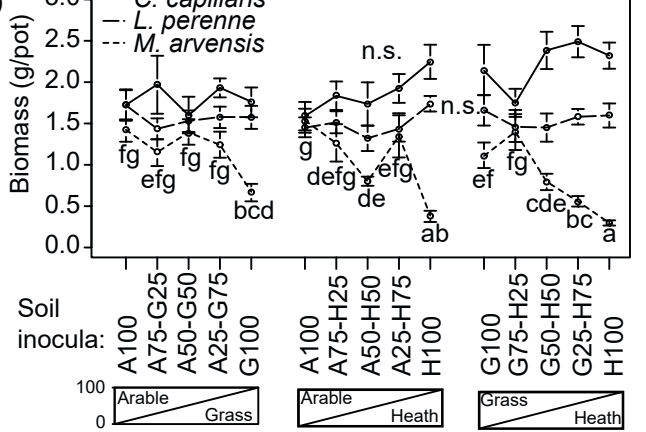

Fig. 3.2. Target $(a, b)$ and ruderal $(c, d)$ species biomass (mean $\pm S E$ ) per inoculum as species groups $(a, c)$ and per species separately $(b, d)$.

Dark grey bars indicate the pure inocula (a, c), the different lines connect species within a replacement series $(b, d)$. Different letters indicate significant differences among the treatments, see Table 3.1 for the overall analyses. 


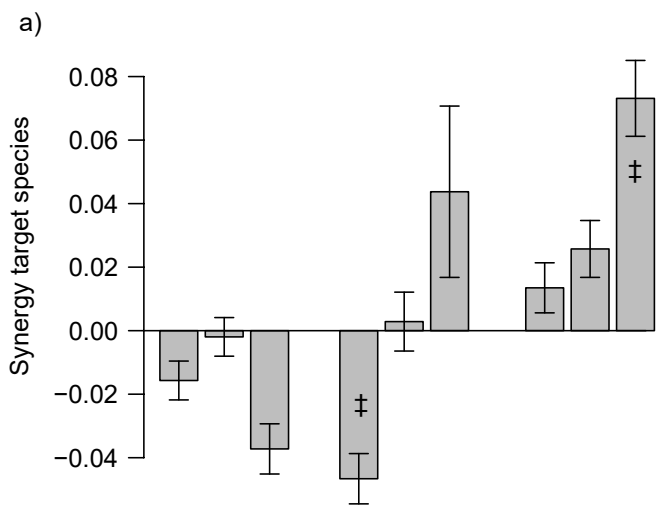

b)
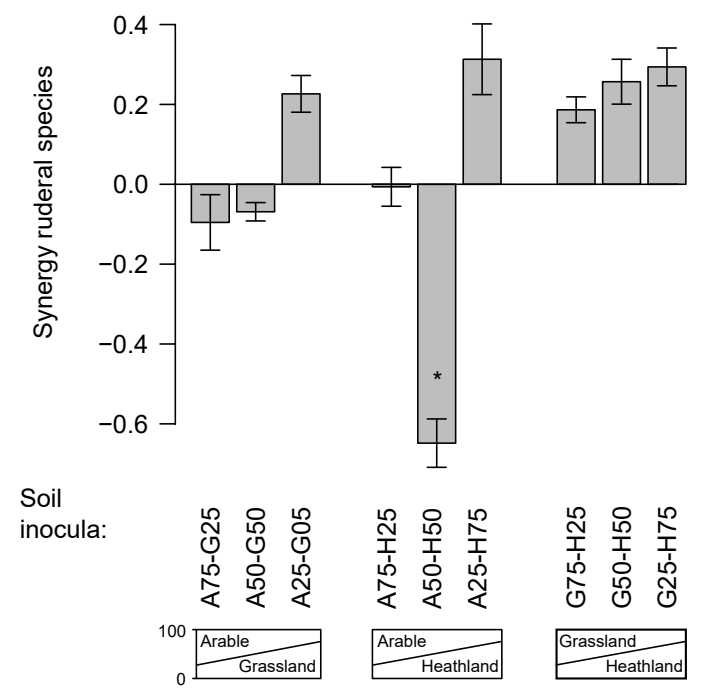

Fig. 3.3. Synergetic effects of mixing inocula.

Shown is the difference in (a) target and (b) ruderal biomass ( $\mathrm{g} / \mathrm{pot}$; mean $\pm \mathrm{SE}$ ) observed in mixed inocula from that expected based on the pure inocula (i.e. dark bars in Fig. 3.2). Asterisk indicates significant difference from zero at $\mathrm{p}<0.05$, $\ddagger$ same except $\mathrm{p}<0.1$.

Mixing of inocula from different field types lead to synergistic effects on the performance of plant groups (Fig. 3.3; Table S3.2). However, only the performance of ruderal species in the A50-H50 treatment was significantly lower than expected based on the pure inocula (Fig. 3.3). Due to the multiple testing correction this difference appears not significant in Fig. 3.2. However, when no such correction is applied, as is appropriate for planned contrasts, ruderal biomass in $\mathrm{A} 50-\mathrm{H} 50$ is significantly lower than in all the other treatments in the replacement series. In addition, there was a trend ( $\mathrm{p}<0.1$; Table S3.2) that G25-H75 and A75-H25 


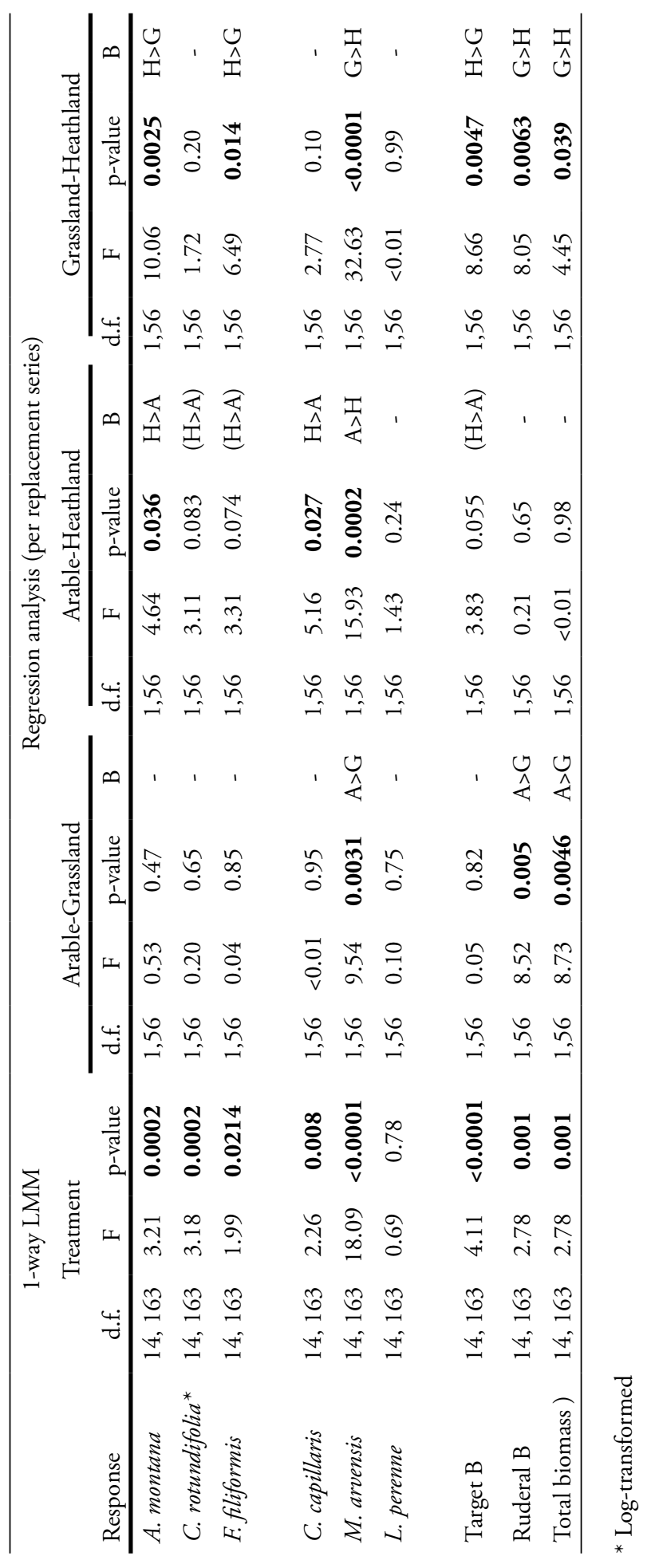


led to higher and lower target species biomass than expected based on the pure inocula. There was no relationship between the synergistic effects of inocula on the ruderal and target species groups (Spearman's rho $=0.078, \mathrm{p}=0.42$ ).

Reponses to changes in inoculum composition were species specific (Fig. 3.2b, d; Table 3.1). The biomass of $M$. arvensis increased with higher amounts of arable soil and decreased with more heathland soil in the inoculum relative to grassland material (Table 3.1; Fig. 3.2d). Each of the three target species responded positively to a higher proportion of heathland material in the inoculum, but the highest biomass of C. rotundifolia was found in the A25-H75 treatment (Fig. 3.2b). More heathland material in the inoculum also led to higher C. capillaris biomass (Fig. 3.1d; Table 3.1). Only L. perenne was not significantly affected by changes in inoculum composition (Fig. 3.1d; Table 3.1).

\section{Discussion}

We found that the composition of the soil inoculum affects the performance of both ruderal and late-successional target plant species. It is known that inoculation with late-successional soil enhances the performance of target plant species (De Deyn et al. 2003, Kardol et al. 2006, Carbajo et al. 2011, Middleton and Bever 2012). We now show that potential synergies among soil inocula exist when material from different successional stages is mixed. In particular, a 50:50 mixture of arable and heathland soil inoculum lead to a lower performance of ruderal plant species than expected. In addition, two trends were observed: a 25:75 grassland-heathland mixture promoted target species biomass, while a 75:25 arable-heathland mixture repressed it relative to the performance expected based on the pure inocula. This shows that there is scope for synergies among soil inocula and thus improving restoration success.

There was no general relationship between the synergistic effects of inoculum mixing on ruderal and target plants, neither positive nor negative. This suggests that synergistic effects only arise in particular combinations of soil inoculum sources and mixing ratios. We found that the mixture of late-successional soil with arable soil most strongly repressed ruderal species growth. This was in line with our hypothesis based on the idea that arable soil harbours high amounts of soil-borne enemies (Korthals et al. 2001, Verschoor et al. 2001, Holtkamp et al. 2008) to which early-successional plants are susceptible (Brown and Gange 1992, Fraser and Grime 1999). However, contrary to our hypothesis this mixture did not significantly improve the growth of target plants. The fact that synergistic 
effects were only detected in particular combinations suggests that general rules based on successional changes may be insufficiently precise for broad application and that synergistic mixtures of inocula may need to be designed specifically for the area under restoration (Eviner and Hawkes 2008).

Plant-soil community interactions have been reported to be non-additive when different soils are mixed (Hendriks et al. 2013, Chapter 5). Soils are inhabited by diverse assemblages of microbial and mesofaunal taxa (Bardgett and van der Putten 2014), but they are also governed by strong competitive and trophic interactions (De Ruiter et al. 1995, Raaijmakers and Mazzola 2016). Soil communities are taxonomically very distinct across ecosystems (Ettema and Wardle 2002, Bardgett and van der Putten 2014). It is therefore likely that when these assemblages are suddenly brought into contact through mixing of inocula that new communities arise that are not simply the weighted sum of the communities in the original inocula (Rillig et al. 2016).

In our experiment plant species showed species-specific responses to the soil inocula. This was particularly true for the ruderal species, where $M$. arvensis responded negatively to heathland inocula, while $C$. capillaris was promoted by higher proportions of heathland inoculum, and $L$. perenne was unresponsive. The target species on the other hand all responded positively to higher proportions of heathland inoculum. This is in line with other inoculation studies (Carbajo et al. 2011, Middleton and Bever 2012) and may reflect their stronger association with soil-borne symbionts such as mycorrhizae (Reynolds et al. 2003). In the future, a wide range of plant species and soil inocula needs to be tested in combination to disentangle differential plant responses to changes in soil community composition and identify which soil taxa drive these responses. This would allow screening for traits of plants and soil biota (see Eviner and Hawkes 2008), and their association with particular habitats, and allow predictions of optimized inocula composition for particular restoration goals.

While the target plants species had 33\% more biomass when soils were inoculated with a high proportion of heathland material compared to no heathland material, they never became the dominant group in the plant community in this experiment (mean relative abundance varied between 5-10\%). In this study, a relatively fertile, recently abandoned arable topsoil was used as a common background soil for the inoculations. It is well known that the outcome of plant-soil interactions depend strongly on environmental context, such as soil type (Bezemer et al. 2006, Eviner 
and Hawkes 2008, Van der Putten et al. 2016). For instance, soil biota tend to have smaller effects on plant performance in more fertile soils (De Deyn et al. 2004, Carbajo et al. 2011, Chapter 2). Under high nutrient conditions plant may be less depended on e.g. mycorrhizae, and better able to defend against antagonists (De Deyn et al. 2004, Van der Bij et al. 2016). In general, there is a need to screen for the effectiveness of soil inoculations across environmental gradients to evaluate their potential (Eviner and Hawkes 2008).

Plant-soil interactions can be mediated by both abiotic and biotic factors (Ehrenfeld et al. 2005). We think that the effect of differences in abiotic conditions among the pots treated with the different inocula was of negligible importance for two reasons. First, due to the dilution of the inocula in the common, relatively fertile, background soil there was limited scope for variation in abiotic conditions, e.g. the $\mathrm{CV}$ was within $1-2 \%$ among treatments for SOM, acidity as well as available $\mathrm{P}$ and $\mathrm{N}$ content. Secondly, the proportion of heathland material in the mixture contributed most to plant performance. However, the heathlands had the lowest concentration of nutrients and the lowest acidity among the sampled inocula. Therefore, if abiotic differences would be the sole driving factor we would expect plant performance to be the worst on $100 \%$ heathland inoculum, which was not observed. In a comparable inoculation study from the same study region both soil abiotic and biotic variables were measured and they showed that plant responses correlated most strongly with biotic drivers, mainly fungal and bacterivorous nematode abundance (Carbajo et al. 2011). Therefore, we propose that the observed differences in plant performance were mainly due to the inoculated soil communities, although we cannot rule out abiotic effects.

We conclude that soil inocula differentially affect the performance of ruderal and late-successional target plant species, also under high soil fertility. Target plant species benefited from inoculation with heathland material, while responses of ruderal species were variable. Mixing inocula from different ecosystems led to synergistic effects for restoration, but this depended highly on the particular combination of inocula applied. As a next step, a broad screen of mixed and unmixed soil inocula needs to be tested across environmental gradients to generally assess the effectiveness of soil inoculation for nature restoration. 


\section{Supplementary information}
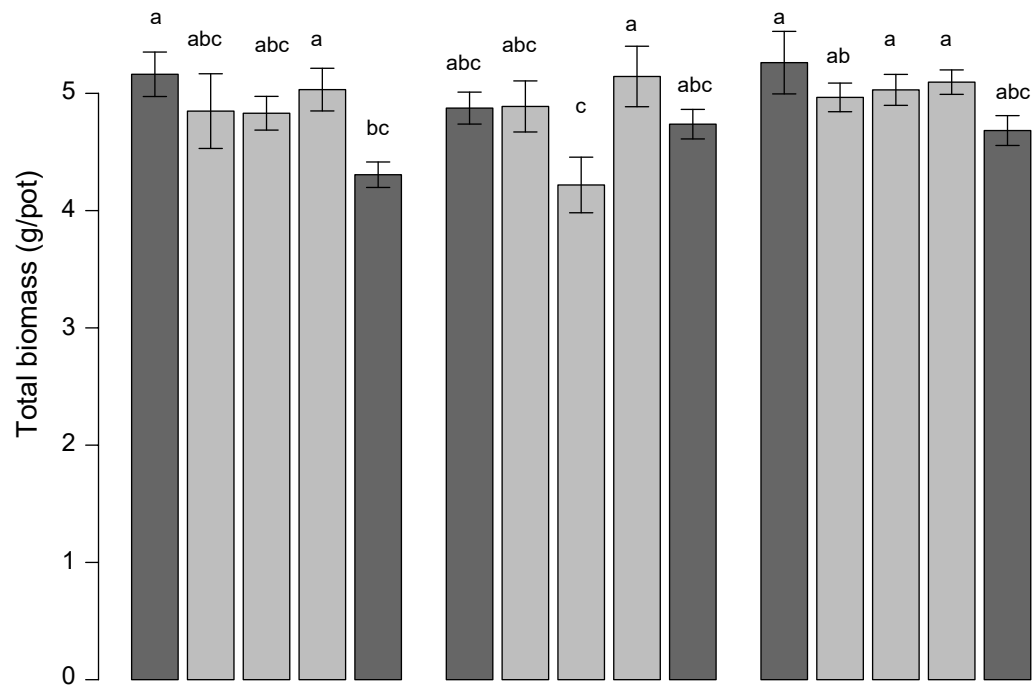

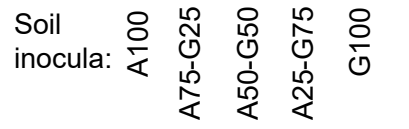

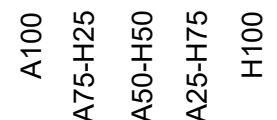

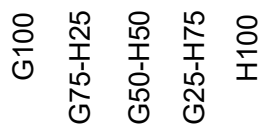

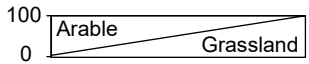

Arable Heathland

Grassland

Heathland

Fig. S3.1. Total shoot biomass (mean $\pm \mathrm{SE}$ ) in response to each of the inoculum treatments.

Different letters indicate significant differences among the treatments. 


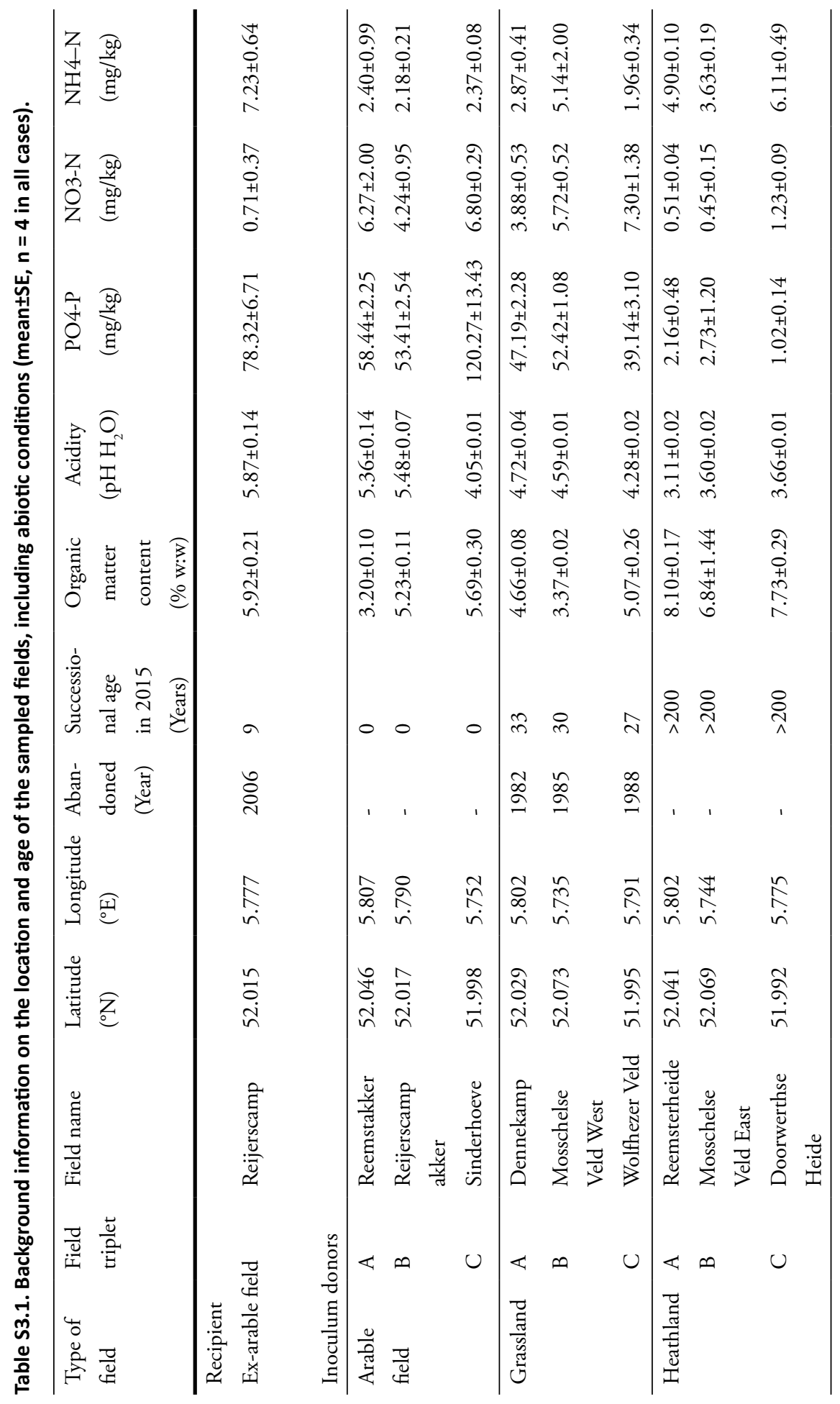


Table S3.2. Results of the planned contrast analyses for synergistic effects of soil inocula mixing on the performance of target and ruderal plant species.

Planned contrasts were tested within LMMs (Table 3.1) to maximise statistical power.

\begin{tabular}{lcccc}
\hline & \multicolumn{2}{c}{ Target spp. } & \multicolumn{2}{c}{ Ruderal spp. } \\
Contrast & $\mathrm{t}_{97}$ & $\mathrm{p}$-value & $\mathrm{t}_{97}$ & $\mathrm{p}$-value \\
\hline A075G025 vs. Pure inoc. & -0.74 & 0.46 & -0.34 & 0.74 \\
A050G050 vs. Pure inoc. & -0.09 & 0.93 & 0.45 & 0.65 \\
A025G075 vs. Pure inoc. & -1.36 & 0.18 & 1.29 & 0.20 \\
A075H025 vs. Pure inoc. & -1.70 & 0.0923 & -0.03 & 0.98 \\
A050H050 vs. Pure inoc. & 0.09 & 0.93 & -2.79 & $\mathbf{0 . 0 0 6 3}$ \\
A025H075 vs. Pure inoc. & 0.47 & 0.64 & 1.02 & 0.31 \\
G075H025 vs. Pure inoc. & 0.49 & 0.62 & 1.17 & 0.25 \\
G050H050 vs. Pure inoc. & 0.83 & 0.41 & 1.23 & 0.22 \\
G025H075 vs. Pure inoc. & 1.77 & 0.0802 & 1.64 & 0.10 \\
\hline
\end{tabular}



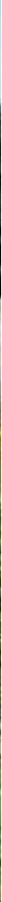

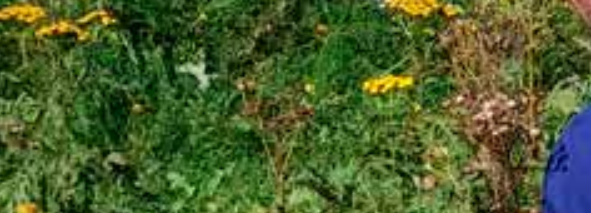
(v) 1.) r.

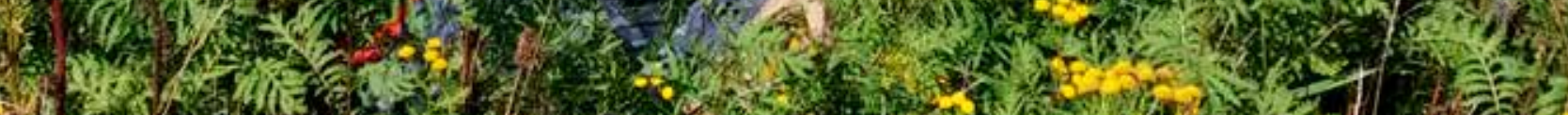
1 to h

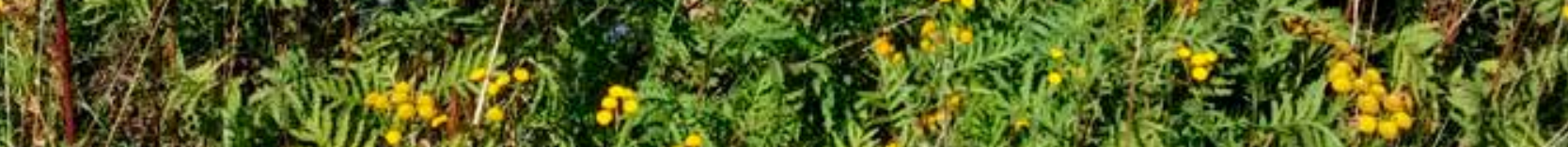

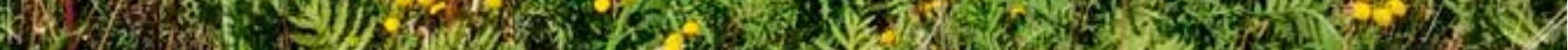


CHAPTER 4

Steering community development - how do sowing and soil inoculation affect above- and belowground communities over two decades?

E.R. Jasper Wubs, Wim H. van der Putten, Simon R. Mortimer, Gerard W. Korthals, Roel Wagenaar, Henk Duyts, Elly Morriën, and T. Martijn Bezemer 


\section{Abstract}

During old-field succession both plant and soil communities strongly change in composition, however, biotic legacies of arable cultivation may prevent unassisted restoration of species-rich communities. It is well known that restoration success is seed limited, but recent studies also show that the soil community needs to be altered for successful restoration. It is, however, unclear whether the key control points for subsequent development lie above- or belowground, and how long interventions influence community composition.

We conducted a long-term field experiment on ex-arable land where, when agricultural practices were ceased, the plant and soil communities were manipulated by seed addition of mid-succession plant species and soil inoculation of mid-succession grassland soil, respectively, in a full factorial design. Nematodes are in important proxy for soil food web functioning and we annually recorded the composition of the nematode as well as the plant community.

The data show that plant communities were mostly influenced by sowing, while the nematode communities responded most strongly to soil inoculation. These effects were persistent for more than twenty and fifteen years, respectively. In addition, sowing also significantly affected nematode community composition in most years and soil inoculation altered plant community composition in the first few years.

We conclude that above- and belowground interventions can independently alter the composition of the plant and nematode communities respectively to a large extend and for prolonged periods (decades). This may offer scope for rescuing and restoring both plant and soil biodiversity. However, as there was no interaction among sowing and soil inoculation their combination did not affect restoration.

\section{Introduction}

Both plant and soil communities change considerably in community composition during old-field succession (Kardol et al. 2005, Van der Wal et al. 2006, Cramer et al. 2008, Bauer et al. 2015, Frouz et al. 2016). Arable cultivation leaves important legacies in the soil in terms of plant seeds and soil community composition (Bekker et al. 1997, Kulmatiski et al. 2006, Kulmatiski and Beard 2011, Tsiafouli et al. 2015) and the initial composition may be an important factor that determines community development after cessation of agricultural practices (Egler 1954, Cramer et al. 2008). It is well known that seed limitation is an important constraint 
for restoration of species-rich grasslands on former arable fields (Hutchings and Booth 1996, Bakker and Berendse 1999, Walker et al. 2004), while recently it has also been shown that the composition of the soil community is a key factor in restoration of plant and soil communities (De Deyn et al. 2003, Kardol et al. 2006, Carbajo et al. 2011, Brinkman et al. 2012, Chapter 2). However, it is unknown whether the trajectory of ecosystem development can be steered primarily by aboveground or by belowground manipulations, or whether they could act synergistically.

In terrestrial ecosystems plants and soil communities greatly influence each other's performance (Wardle et al. 2004, Bardgett and Wardle 2010). Plants influence soil communities through e.g. root exudation and litter inputs (Ehrenfeld et al. 2005, Van der Putten et al. 2013). Plant species differ in these traits and as a consequence plant species in the field are known to harbour their own specialized local soil communities (Bezemer et al. 2010, Viketoft et al. 2011). Soil communities in turn affect the performance of plants and the composition of plant communities. For instance, root herbivores selectively feed on early-successional palatable species and may enhance succession (Brown and Gange 1992, Fraser and Grime 1999). Soil-borne plant pathogens can suppress dominant species (Packer and Clay 2000, Bever et al. 2015) and mycorrhizal fungi and growth promoting rhizobacteria may improve the nutrition, defence and ultimately fitness of particular plant species in the community (Van der Heijden et al. 2008, Bardgett and van der Putten 2014, Raaijmakers and Mazzola 2016).

Most natural ecosystems are seed limited and this is particularly true of earlysuccessional, disturbed systems (Hutchings and Booth 1996, Turnbull et al. 2000, Zobel et al. 2000). In addition, seed sowing can have strong effects on plant community composition, diversity and functioning (Turnbull et al. 2000, Bezemer and Van der Putten 2007, Kardol et al. 2008) and can be a key step in overcoming the legacies created by arable farming (Hutchings and Booth 1996, Kardol et al. 2008, Prach et al. 2014). On the other hand, soil inoculation has the potential to introduce both plant seeds as well as their associated soil communities (Bullock 1998, Kardol et al. 2009, Kiehl et al. 2010), and the soil community can play a key role in determining the direction of plant community development (Chapter 2). It is currently unclear whether interventions targeted at plants or the soil community may provide the key control point on subsequent community development. The co-introduction of both plant and soil communities is thought to exert a positive feedback on their own establishment (Kardol et al. 2006, Chapter 2), while 
hampering early successional ruderal plants through competition and negative indirect soil feedback, which suggests that a combination of treatments would be most successful. In addition, it is unknown how long these interventions affect plant and soil community composition, particularly for soil inoculation (Marrs 2016).

Here we study how above- and belowground interventions, respectively sowing plant seeds and soil inoculation, influence the development of both the vegetation and the soil community. We conducted an experiment on a field that had been under arable cultivation until the year before the treatments were implemented and that was acquired by a nature managing organization with the objective of restoring it to a species-rich grassland. In the experiment we factorially combined sowing and inoculation treatments and monitored the resulting plant and nematode communities for 20 years. Nematodes are a diverse group of soil fauna, sensitive to environmental changes, that occur at most trophic levels in the soil food web, and a such are a key component of the soil community (Ferris et al. 2001, Yeates 2003, Neher 2010). Both the plant seeds as well as the soil inocula were representative of the mid-succession stage within our study system. We investigated how the development of the plant and nematode communities was influenced by above- and belowground intervention.

We hypothesized that sowing most strongly influences plant community development, while soil inoculation is the primary determinant of soil community composition. In addition, we expected that sowing would have a longer lasting effect than soil inoculation, since the soil community would have to invade an established resident community, while the experiment started from bare soil, and hence a plant cover still had to redevelop after cessation of arable cultivation. Finally, we expected that the combination of both sowing and soil inoculation with mid-succession communities would lead the most rapid community development. Therefore, plots that were both sown and inoculated were expected to be most similar to the mid-succession grassland used as a donor.

\section{Methods}

\section{Site characteristics \& experimental setup}

The field experiment was established in spring 1996 on a former arable field in the Netherlands ("Mossel", GPS: $52.07^{\circ} \mathrm{N} 5.75^{\circ} \mathrm{E}$ ). The site is situated on a sandy loam soil and has been in agricultural production until autumn 1995 (last crop was maize; Van der Putten et al. 2000). A fence was erected around the experiment to keep large grazers out and the soil was harrowed and equalized in April 1996. 
The experiment was implemented as a randomized block design with 20 plots $(2 \times 2 \mathrm{~m})$ established in five replicate blocks and the sowing and soil inoculation treatments were fully crossed in the design.

At the start of the experiment, half of the plots were treated with soil inocula from a nearby $(<1 \mathrm{~km})$ mid-succession grassland (Mosschelseveld, GPS: $52.06^{\circ} \mathrm{N} 5.75$ ${ }^{\circ} \mathrm{E}$ ) that had been undergoing natural secondary succession since 1985 (10 years at the start of the experiment) and was similar in abiotic conditions (Kardol et al. 2005, Van der Wal et al. 2006). Soil was collected from the top $15 \mathrm{~cm}$ of the donor site, gently mixed and was applied in a thin layer $\left(10 \mathrm{~L} \mathrm{plot}^{-1}\right)$ and harrowed in at a depth of $5 \mathrm{~cm}$ in April 1996. Subsequently four turf monoliths (25 x $25 \times 25 \mathrm{~cm})$ of soil from the same donor site were introduced in to the four corners of each plot in November 1996.

Half the plots were sown with 15 mid-succession plant species, equally representing grasses, legumes and non-legume forbs (for selection criteria see Van der Putten et al. 2000). The species were Agrostis capillaris L., Anthoxanthum odoratum L., Festuca rubra L., Phleum pratense L., Poa pratensis L. (all Poaceae), Hypericum perforatum L. (Hypericaceae), Hypochaeris radicata L. (Asteraceae), Linaria vulgaris Mill., Plantago lanceolata L. (both Plantaginaceae), Tanacetum vulgare L. (Asteraceae), Lotus corniculatus L., Trifolium arvense L., T. dubium Sibth., T. pratense L., and Vicia cracca L. (all Fabaceae) and they were sown at a density of 500 seeds $\mathrm{m}^{-2}$ for grass species and 100 seeds $\mathrm{m}^{-2}$ for legume and other forb species in April 1996. The seeds were either hand-sown or mixed with sand/soil to facilitate equal spreading. Subsequently, the plots were harrowed to bring the seeds into the surface layer. The experiment has been ongoing since 1996 until present. At the end of each growing season, the aboveground vegetation of all plots and border rows was mown and removed in September.

\section{Measurements}

Annually the composition of the vegetation and the nematode community composition were recorded. Percent cover of all plant species was recorded each year in the central 1x1 m square of each plot in August 1997-2016 (nomenclature following Van der Meijden 2005). Plant aboveground biomass in all plots was clipped in September 1998-2016 in a 25 x $25 \mathrm{~cm}$ square in each plot and dried $\left(70{ }^{\circ} \mathrm{C}, 48 \mathrm{~h}\right)$ and weighed. Twenty-four soil cores $(0-15 \mathrm{~cm}$ depth, diameter 17 $\mathrm{mm}$ ) were collected from each plot in August (1998-2013) and nematodes were extracted from $100 \mathrm{~g}$ fresh soil (Oostenbrink 1960), heat-killed at $90{ }^{\circ} \mathrm{C}$, and fixed 
in $4 \%$ formaldehyde solution. Subsequently the total number of nematodes was counted and at least 100 were identified to family level (following Bongers 1988). In cases where there were less than 100 nematodes, all nematodes were identified. At the start of the experiment, we sampled nematode community composition in both the experimental and the donor site (Table S4.1). In each field seven 10x10 m plots were haphazardly selected and 24 soil cores were taken and analysed as before for each of these plots. In addition, we recorded plant and nematode community composition in the donor site in 2011. For this purpose, as transect was placed in the field and three $1 \times 1 \mathrm{~m}$ plots, separated by at least $100 \mathrm{~m}$, were sampled using the same methods as described before.

\section{Data analysis}

Data analysis on communities was conducted on the relative abundances of each taxon. For each plot in each year we calculated the diversity of the plant and nematode community using the Shannon index and we calculated the similarity of these communities to the donor plant or soil community, respectively. Similarity was defined as 1 minus the Bray-Curtis distance on log-transformed relative abundances (Bray and Curtis 1957). We calculated the similarity to each of the three plots in the donor site separately and then averaged over these values to obtain one similarity value per plot in the experiment per year.

We used principal components analyses (PCA) to visualize differences in community composition, on log-transformed relative abundances. To visualize the effects of the experimental treatments we conducted partial redundancy analyses (partial RDA) for both communities separately, where the year effect was partialled out. We modelled the community responses as a linear function of the sowing and soil inoculation treatment, their interaction and interactions with time to allow the treatment effects to vary over years. This is the same as a Principal Response Curve analysis (Van den Brink and Ter Braak 1999), except that the regression equations were not coded to take one treatment as a reference treatment (see also (Pierik et al. 2011). We tested for the significance of the experimental treatments, time and their interactions on community composition using Multiple-Response Permutation Procedures (MRPPs; Anderson 2001). Permutations were restricted within blocks and were further constrained to take into account the repeated observations within the plots (Legendre and Legendre 2012). We repeated these MRPPs to test for differences per year, in which case permutations were restricted within blocks. 
Univariate responses were analysed using Generalized Additive Mixed Models (GAMM), where temporal patterns over years were modelled using cubic regression splines (Wood 2006). Sowing and soil inoculation and their interaction were included as fixed effects. Block and year were included as random effects to account for spatial and temporal sampling effects. Interactions between the treatments and temporal patterns over years were tested by comparing two models, one with a regression spline per treatment and another where one common regression spline was used for all treatments. These models were compared using likelihood ratio tests (Wood 2006). Linear Mixed Models (LMMs) were used to analyse univariate data per year, with the same fixed effects as above and a random effect for blocks.

All analyses were conducted in R 3.3.1 (R Core Team 2016). Multivariate analyses were done in the vegan 2.4-1 package (Oksanen et al. 2016), GAMMs were analysed using the mgcv 1.8-12 package (Wood 2006) and LMMs using nlme (Pinheiro et al. 2016). Model assumptions were checked graphically. In case of violations the response variable was either log-transformed (plant biomass data) or heteroscedasticity was modelled explicitly using generalized least squares (Pinheiro and Bates 2000, Zuur et al. 2009).

\section{Results}

Both plant and nematode communities changed strongly over time (Table 4.1; Fig S4.1), but the sowing and soil inoculation treatments had contrasting effects on the above- and belowground communities (Fig. 4.1). For the vegetation, sowing had the strongest effect on community composition (Table 4.1), which lasted for the full 20 years of monitoring (Fig. 4.1a,b,c; Table S4.2). Soil inoculation also significantly affected the vegetation during the first three years of the experiment (Fig. 4.1a; Table S4.2). In the final years (2013-2016), the composition of the vegetation in unsown-inoculated treatment became more similar to the sown treatments than the unsown treatment without soil inoculation (Fig. 4.1b,c; Fig. S4.1), but this was not significant (Table S4.2).

For the nematodes, the community composition differed between the experimental and donor site at the start of the experiment (Table S4.1; MRPP Pseudo-F = $\left.22.19, \mathrm{p}=0.011, \mathrm{R}^{2}=73.5 \%\right)$. As a result, soil inoculation most strongly altered nematode community composition during the experiment (Fig. 4.1d; Table 4.1) and led to an increasingly diverging community composition in the first seven years (Fig. 4.1e,f). This soil inoculation effect persisted into later years (Fig. 4.1e,f; 

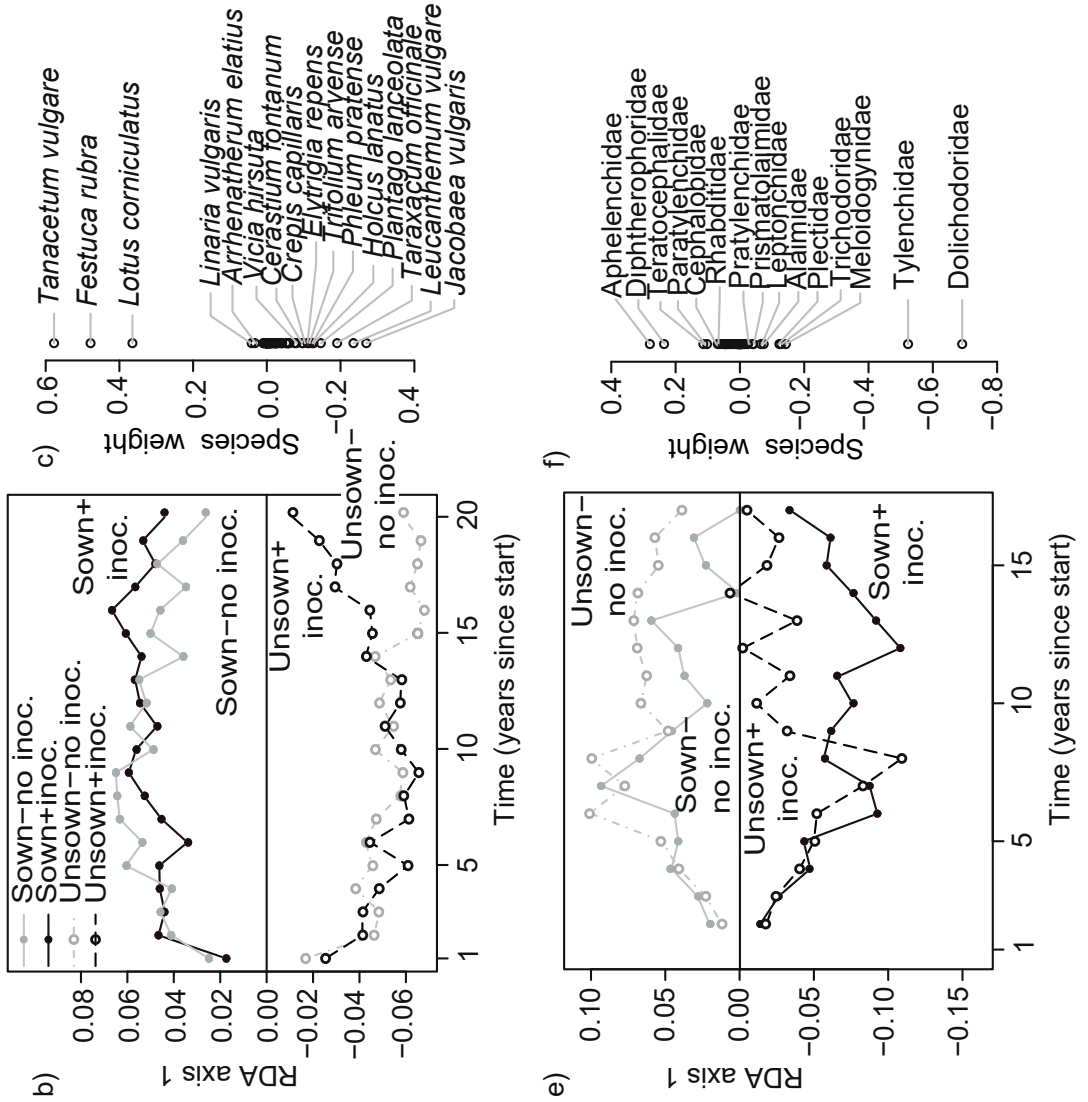

$\widehat{\Omega}$

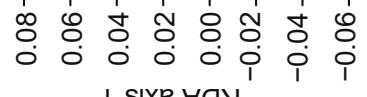

l $\quad$ s!xe $\forall a d$
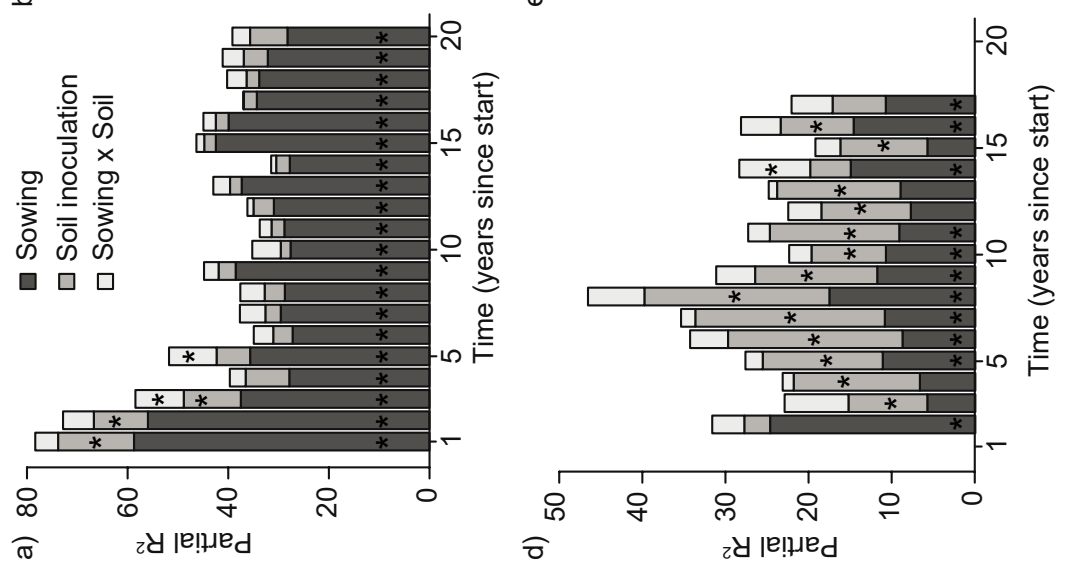

Fig 4.1. Changes in plant (a-c) and nematode (d-f) community composition due to the experimental treatments over 20 years $\left(t_{0}=1996\right)$.

Plots were sown with a seed mixture of 15 mid-succession plant species and inoculated with midsuccession soil in a full factorial design. Shown are the yearly partial $R^{2}$ values $(\%$;,$d)$ for the 


\section{Fig. 4.1. caption continued.}

effect of the experimental treatments on community composition for the duration of monitoring. Stars in each bar indicate that it explained a significant portion of the variation, see Table S4.2 and S4.3 for statistical tests. In addition, partial RDA analyses (b, e) on community composition show the direction of community changes due to the experimental treatments after accounting for the difference across years (partialled out in RDA; see Fig S4.1 for unconstrained ordinations). The first RDA axes explain $14.9 \%$ and $4.1 \%$ of the variation for plant and nematode community composition, respectively, while the partialled out year effects accounted for $21.2 \%$ and $54.2 \%$. Taxa weights in the ordinations are shown on the vertical one-dimensional plots (c, f) and show changes the relative abundance of each taxon across the RDA axes. The sign of the weight indicates the direction in which their relative abundance increases. For clarity, only taxa with the best fit to the ordination axes are shown by name. Inoc. = soil inoculation.

Table S4.3), although the communities slowly converged until the soil inoculation treatment effect became non-significant in the final year of monitoring (2013; Table S4.3). The sowing treatment also affected nematode community composition and this was particularly evident in later years (Fig. 4.1d,e,f; Table S4.3). In neither the vegetation nor the nematode community were there strong interactions among the sowing and soil inoculation treatments (Fig. 4.1; Table 4.1).

The vegetation in the unsown plots gradually became more similar to the donor community over time, while the sown plots first declined in similarity and only
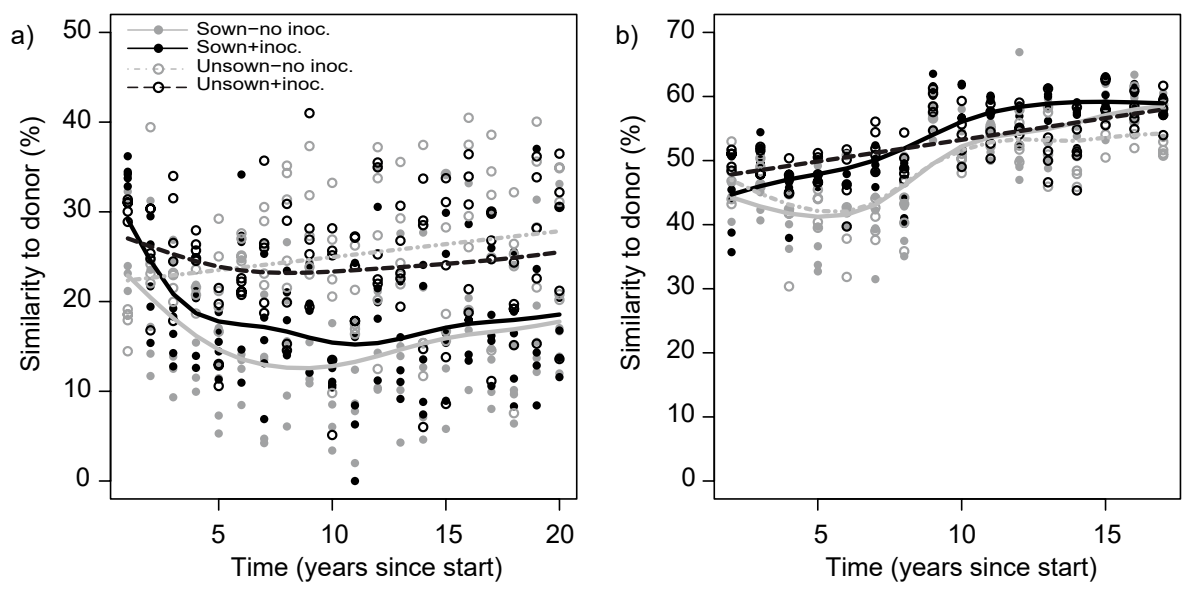

Fig. 4.2. Similarity of the vegetation (a) and the nematode (b) community composition to the composition of the donor site (recorded in 2011) over time and for each of the four experimental treatments.

The lines show the estimated relationships based on the GAMM analyses (see Table 4.2). Inoc. = soil inoculation. 
after ten years (2007 onward) they gradually started to become more similar to the donor community (Fig. 4.2a; Table 4.2). As a result, sown plots generally had a lower similarity to the donor than unsown plots (Fig. 4.2a). The sown species accounted for most of the plant cover throughout the experiment, particularly Tanacetum vulgaris, Festuca rubra and Lotus corniculatus (Fig. 4.1c; S4.1a). Soil inoculation initially increased the similarity of the vegetation to the donor community in the first year (Fig. 4.2a; Table S4.4), but in later years this was no longer the case. For nematodes, inoculated plots were more similar to the donor community than uninoculated plots (Fig. 4.2b; Table 4.2; Table S4.5) and similarity to the donor community increased over time in all treatments (Fig. 4.2b).

Plant biomass declined slowly over time and was significantly higher $(-50 \%)$ in sown plots; soil inoculation did not affect plant biomass (Fig. S4.2a, Table S4.6). Plant diversity was very similar among the treatments during the first years of the experiment, but subsequently the diversity of sown plots declined, while in unsown plots diversity did not change substantially (Fig. S4.2b, Table S4.6). Soil inoculation led to a moderate increase in plant diversity, particularly during the middle years of the experiment (between 8-15 years after the start; Fig. S4.2b). Soil inoculation led to somewhat higher nematode diversity and in general nematode diversity increased over time (Fig. S4.2c; Table S4.6).

Table 4.1. Overall MRPP tests for differences in community composition.

\begin{tabular}{|c|c|c|c|c|c|c|c|c|}
\hline \multirow[b]{2}{*}{ Term } & \multicolumn{4}{|c|}{ Vegetation } & \multicolumn{4}{|c|}{ Nematodes } \\
\hline & d.f. & Pseudo-F & p-value & $\begin{array}{l}\mathrm{R}^{2} \\
(\%)\end{array}$ & d.f. & Pseudo-F & p-value & $\begin{array}{l}\mathrm{R}^{2} \\
(\%)\end{array}$ \\
\hline Year & 19 & 12.69 & $0.001^{* * *}$ & 28.9 & 15 & 37.96 & $0.001^{* * *}$ & 61.5 \\
\hline Sowing & 1 & 140.45 & $0.001^{* * *}$ & 16.8 & 1 & 19.50 & $0.001^{* * *}$ & 2.1 \\
\hline Soil transfer & 1 & 8.10 & 0.257 & 1.0 & 1 & 34.38 & $0.001^{* * *}$ & 3.7 \\
\hline Yr x sow & 19 & 3.98 & $0.001^{* * *}$ & 9.1 & 15 & 1.35 & 0.147 & 2.2 \\
\hline Yr x soil & 19 & 1.35 & $0.002^{* *}$ & 3.1 & 15 & 0.82 & 0.518 & 1.3 \\
\hline Sow x soil & 1 & 7.14 & 0.285 & 0.9 & 1 & 1.91 & 0.418 & 0.2 \\
\hline Year $\mathrm{x}$ sow $\mathrm{x}$ soil & 19 & 0.93 & 0.074 & 2.1 & 15 & 0.80 & 0.506 & 1.3 \\
\hline Residuals & 320 & & & 38.3 & 256 & & & 27.7 \\
\hline
\end{tabular}


Table 4.2. Results of GAMM analyses on similarity to the donor community.

Temporal changes were modelled using cubic regression splines (indicated as $\mathrm{S}($ year)), and models with separate splines per treatment (time $\mathrm{x}$ treatment interaction) or one common spline were compared using the likelihood ratio test and AIC values.

\begin{tabular}{|c|c|c|c|c|c|c|}
\hline \multirow[b]{2}{*}{ Terms } & \multicolumn{3}{|c|}{ Vegetation similarity } & \multicolumn{3}{|c|}{ Nematode similarity } \\
\hline & $\Delta \mathrm{AIC}^{*}$ & LR & $\mathrm{p}$-value & $\triangle \mathrm{AIC}$ & LR & $\mathrm{p}$-value \\
\hline \multirow[t]{2}{*}{$S($ year $) \times$ treatment } & -9.0 & 20.96 & 0.0019 & -5.5 & 17.48 & 0.0077 \\
\hline & d.f. & $\mathrm{F}$ & $p$-value & d.f. & $\mathrm{F}$ & $\mathrm{p}$-value \\
\hline Sowing & 1 & 112.17 & $<0.0001$ & 1 & 1.39 & 0.240 \\
\hline Soil & 1 & 7.62 & 0.006 & 1 & 47.57 & $<0.0001$ \\
\hline SxS & 1 & 6.50 & 0.01 & 1 & $<0.00$ & 0.974 \\
\hline$S($ year $)$ & - & - & - & - & - & - \\
\hline$S$ (year): HD-0 & 3.41 & 5.80 & 0.0006 & 4.92 & 35.55 & $<0.0001$ \\
\hline S(year): HD-S & 4.56 & 5.59 & 0.0001 & 3.71 & 32.22 & $<0.0001$ \\
\hline$S$ (year): NC-0 & 1.00 & 6.71 & 0.01 & 5.10 & 18.66 & $<0.0001$ \\
\hline$S$ (year): NC-S & 2.42 & 0.83 & 0.314 & 1.00 & 40.06 & $<0.0001$ \\
\hline
\end{tabular}

${ }^{*}$ positive $\triangle \mathrm{AIC}$ values indicate better fit for model without interaction between the treatments and time, negative values support the model with interaction.

\section{Discussion}

Our results demonstrate that both seed addition and soil inoculation can influence plant and soil community development. Sowing had the strongest influence on plant community composition, while soil inoculation exerted the dominant control over the composition of the nematode community. Remarkably, there were no clear interactions among sowing and soil inoculation on either above- or belowground community composition.

Our results show that sowing at the start of grassland restoration can have a lasting effect on plant community composition, which persisted for at least 20 years. More importantly, we show for the first time that soil inoculation can also have persistent effects, in particular on belowground community composition. During the first eight years, the inoculated soil communities increasingly diverged from the uninoculated communities and significant differences in soil community composition were detected more than 15 years post-inoculation. 
Soil inoculation primarily affected nematode community composition and there was no interaction with sowing. These data highlight that there is scope for the manipulation of soil communities independent of the plant community. It has been shown that soil macro-invertebrates and nematode communities can follow successional trajectories that are independent from the successional development of the plant community (Berg and Hemerik 2004, Kardol et al. 2005). This could be because soil communities can be different in taxonomic composition while their functioning, e.g. with respect to elemental cycling and food web structure, is the same (Malý et al. 2000, Bezemer et al. 2010). In general, plant and soil communities are thought to be tightly coupled (Bardgett and Wardle 2010, Scherber et al. 2010), however, our data shows that at least for the nematode community this is not the case. Apart from nematodes, it would be interesting to explore which other functional and taxonomic groups of soil bacteria, fungi, or arthropods can be steered independently from the plant community. This may offer opportunities to selectively manipulate parts of the soil food web and thereby optimizing plant-soil interactions for nature restoration and perhaps soil-borne disease suppressiveness (Raaijmakers and Mazzola 2016).

In the course of the experiment, the strength of the treatment effects changed. For instance, soil inoculation did initially influence plant community composition and made the vegetation more similar to the donor community, but this effect dissipated after a few years. Some plant propagules are expected to be introduced with the soil inoculum (Bullock 1998, Chapter 2), but these were probably outcompeted by the ruderal species typical of arable soil seed banks (Hutchings and Booth 1996, Bekker et al. 1997, Kardol et al. 2008). Furthermore, while soil inoculation had the strongest effect on the nematode community, sowing also influenced nematode community composition and this effect became more apparent with time. Nematode communities depend importantly on plants as their hosts (plant-feeding nematodes) or for inputs of organic C (mainly bacterivorous nematodes), and the sowing effect on nematode community composition may have been indirectly mediated through bottom-up changes in resource quality (Neher 2010, Scherber et al. 2010, Viketoft et al. 2011).

Previous research suggested that the co-introduction of both seeds and soil community can enhance community development (Kardol et al. 2006, Carbajo et al. 2011, Van der Putten et al. 2013, Chapter 2). However, contrary to this hypothesis, in our long-term field experiment the combination of sowing midsuccession plant species and inoculating a mid-successional soil community did 
not lead to synergistic effects on community composition or similarity to the donor community. Other work has suggested that the site receiving the inocula should have the appropriate abiotic conditions to allow colonization from the inoculum (Kardol et al. 2009). However, the abiotic conditions of the donor site and the experimental site were rather similar and soil inoculation had a profound and lasting effect on nematode community composition. Therefore we may assume that the abiotic environment was highly appropriate for the inoculated community. Instead, the difference in community composition between the donor and the recipient site may have been relatively small and thus a soil community somewhat similar to the resident community may have been inoculated. Comparison of different inocula showed that an inoculum from a late-successional dry heathland system, that had been allowed to develop for hundreds of years, was more effective than an inoculum from a mid-succession grassland (Chapter 2). In addition, in case the topsoil is left intact, as was done in the present study, rather than removed entirely, soil inoculation has been shown to be less effective (Carbajo et al. 2011, Chapter 2). Finally, the present experiment was conducted at a much smaller spatial scale (4 $\mathrm{m}^{2}$ plots vs approximately 0.5 ha areas in Chapter 2 ), which may have reduced the likelihood of successful introduction of soil taxa from the inoculum.

Our results show that sowing strongly affected aboveground community composition and may thus be a key management tool to restore natural communities (Pywell et al. 2002, Foster et al. 2007, Kardol et al. 2008). Indeed, other studies have shown that sowing can have profound impacts on the vegetation (e.g. Bezemer and Van der Putten 2007, Kardol et al. 2008), although grazing and cutting may be of overruling importance (Warren et al. 2002). However, in our case sowing did not improve restoration success as both the diversity as well as the similarity to the donor community was lower in the sown plots. Sowing led to a rapid establishment of the sown species, but this prevented colonization by other species in this relatively productive field (approximately $400-800 \mathrm{~g} \mathrm{~m}^{-2}$ ). In fact, sowing may have selected for the dominance of a number of productive species, as sowing increased aboveground biomass throughout the experiment. These results are in line with old-field succession where mid-succession species can prevent subsequent vegetation development once they have gained dominance (Cramer et al. 2008, Bartha et al. 2014). Sowing may therefore be a highly effective tool, but its success in terms of restoration will depend importantly on the identity of the selected species and their interactions with competing plants and the soil community (Eviner and Hawkes 2008, Kardol and Wardle 2010). Soil inoculation also can be a powerful tool to restore natural vegetation (Chapter 2), but in our 
study its clearest impact was on belowground community composition. As soil inoculation increased both the diversity of the nematode community and its similarity to the donor community it may be an important tool in the establishment of belowground reserves of biodiversity (Turrini and Giovannetti 2012).

In conclusion, we found that over a span of twenty years sowing most strongly altered plant community development, while soil inoculation drove differences in nematode community composition. In addition, sowing also influenced nematode community composition, particularly in the longer term. The combination of sowing and soil inoculation did not lead to the highest similarity to the target community or diversity. Our experiment highlights that plant and nematode community development can be affected independently, for a large part, by aboveand belowground interventions respectively. 


\section{Supplementary information}
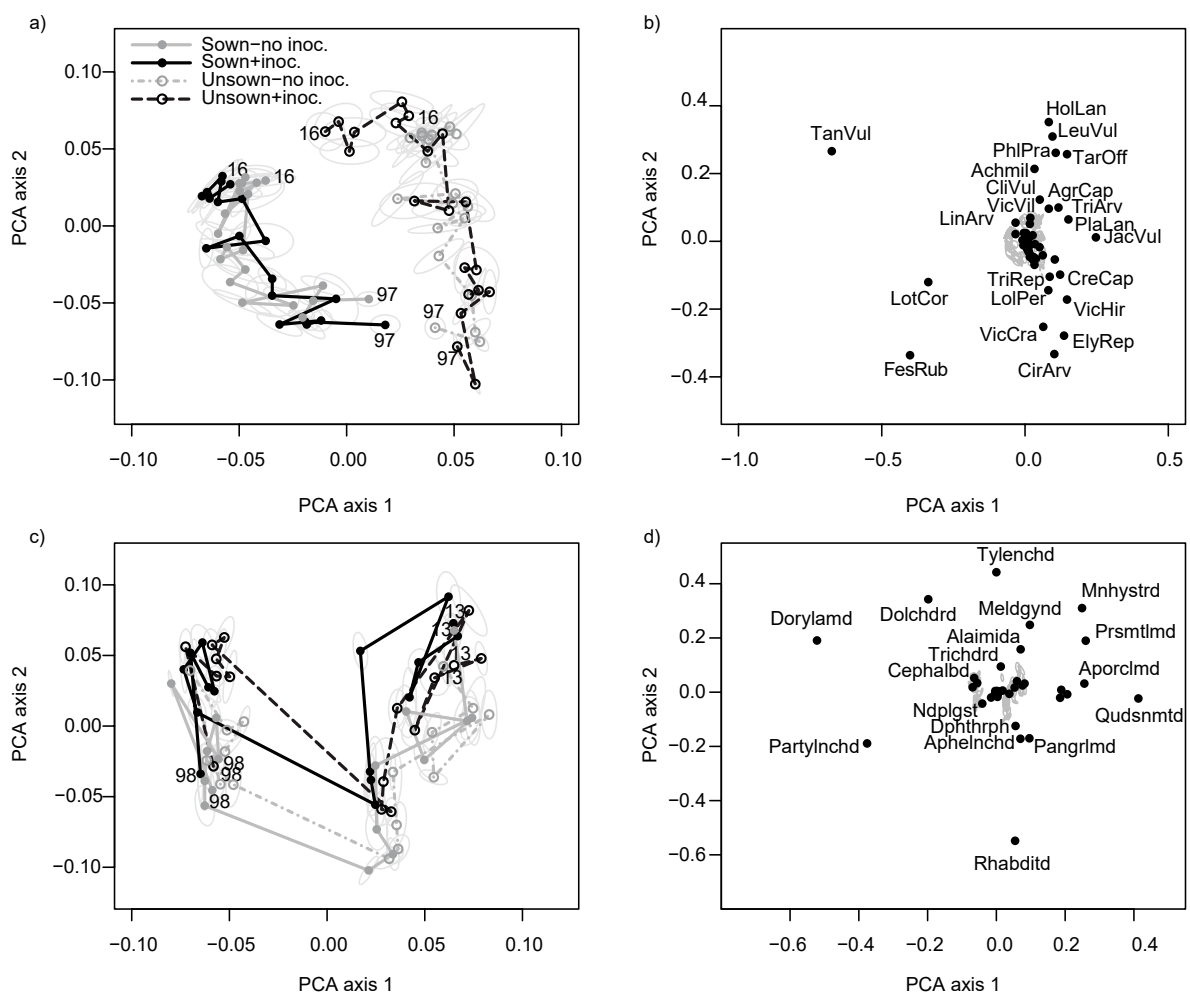

Fig. S4.1. PCAs for vegetation (a,b; axis explain $19.2 \% \& 9.5 \%)$ and nematode (c, d; axes explain $36.1 \%$ \& $8.9 \%$ ) community composition in the field experiment over time.

The left panels (a, c) show the means (dots) and SEs (grey ellipses) of the treatments (MRPP analyses in Table S4.2 \& S4.3) in each year. The lines connect the trajectory of each treatment through time, and the first and the last year of monitoring are indicated by the last two numerals of that year. The right panels (b, d) shows the taxon scores on the same PCA ordination plot. For clarity, only taxa with the best fit to the ordination axes are shown by name. Abbreviations are: Inoc. $=$ soil inoculation, in $\mathrm{b})$ AchMil $=$ Achillea millefolium, AgrCap $=$ Agrostis capillaris, CirArv $=$ Cirsium arvense, CliVul $=$ Clinopodium vulgare, CreCap $=$ Crepis capillaris, ElyRep $=$ Elytrigia repens, FesRub $=$ Festuca rubra, HolLan $=$ Holcus lanatus, JacVul = Jacobaea vulgaris, LeuVul $=$ Leucanthemum vulgare, LinArv $=$ Linaria vulgaris, LolPer $=$ Lolium perenne, LotCor $=$ Lotus corniculatus, $\mathrm{PhlPra}=$ Phleum pratense, PlaLan $=$ Plantago lanceolata, $\mathrm{TanVul}=$ Tanacetum vulgare, TarOff $=$ Taraxacum officinale, TriArv $=$ Trifolium arvense, $\mathrm{VicCra}=$ Vicia cracca, VicHir $=$ Vicia hirsuta, $\mathrm{VicVil}=$ Vicia villosa , and in d) Alaimida $=$ Alaimidae, Aphelnchd $=$ Aphelenchidae Aporclmd $=$ Aporcelaimidae, Cephalbd $=$ Cephalobidae, Dphthrph $=$ Diphtherophoridae, Dolchdrd $=$ Dolichodoridae, Dorylamd $=$ Dorylaimidae, Meldgynd $=$ Meloidogynidae, Mnhystrd $=$ Monhysteridae, Ndplgst $=$ Neodiplogasteridae, Pangrlmd $=$ Panagrolaimidae, Partylnchd $=$ Paratylenchidae, Prsmtlmd = Prismatolaimidae, Qudsnmtd = Qudsianematidae, Rhabditd $=$ Rhabditidae, Trichdrd $=$ Trichodoridae, Tylenchd $=$ Tylenchidae . 

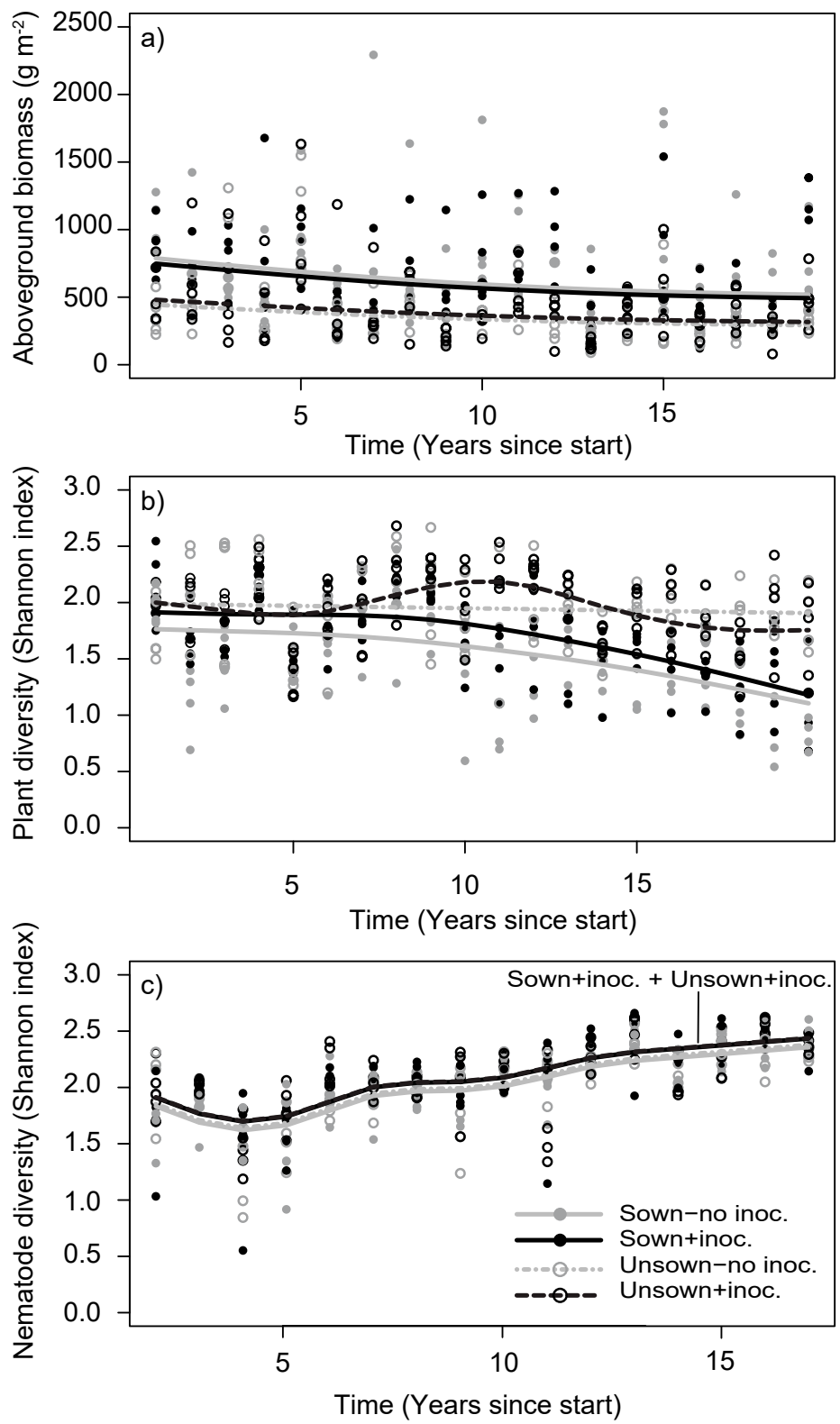

Fig. S4.2. Temporal changes in aboveground plant biomass (a) and diversity of plants (b) and nematodes (c) in response to the experimental treatments.

The lines show the estimated relationships based on the GAMM analyses, see Table S4.6 for statistical analysis. Inoc. $=$ soil inoculation. 
Table S4.1. Abundance (no. $100 \mathrm{~g} \mathrm{~g}^{-1}$ soil) of nematode families in the experimental site and the soil inoculum donor site at the start of the experiment.

Data taken from seven samples in each field in April of 1996. Means and standard errors (SE) are shown.

\begin{tabular}{llcccc}
\hline & & \multicolumn{2}{c}{ Experimental site } & \multicolumn{2}{c}{ Donor site } \\
Taxon & Feeding group* & Mean & SE & Mean & SE \\
\hline Anguinidae & Fungal-feeding & 19 & 10 & 13 & 6 \\
Aphelenchidae & Fungal-feeding & 36 & 14 & 289 & 31 \\
Cephalobidae & Bacterial-feeding & 417 & 53 & 1156 & 240 \\
Diphtherophoridae & Fungal-feeding & 0 & 0 & 29 & 12 \\
Dolichodoridae & Plant-feeding & 84 & 29 & 436 & 82 \\
Dorylaimidae & Omnivore & 131 & 36 & 142 & 39 \\
Mononchidae & Carnivorous & 1 & 1 & 13 & 4 \\
Paratylenchidae & Plant-feeding & 490 & 61 & 51 & 38 \\
Plectidae & Bacterial-feeding & 33 & 11 & 195 & 36 \\
Pratylenchidae & Plant-feeding & 154 & 60 & 63 & 21 \\
Prismatolaimidae & Bacterial-feeding & 11 & 7 & 82 & 20 \\
Rhabditidae & Bacterial-feeding & 243 & 37 & 191 & 33 \\
Teratocephalidae & Bacterial-feeding & 8 & 7 & 35 & 11 \\
Trichodoridae & Plant-feeding & 0 & 0 & 26 & 10 \\
Tylenchidae & Plant-feeding & 75 & 20 & 110 & 19 \\
& & 1701 & 164 & 2830 & 388 \\
Total & & & & &
\end{tabular}

* Based on Yeates et al. (1993). 
Table S4.2. Results of MRPP tests for differences in plant community composition per year.

\begin{tabular}{lcccccc}
\hline & \multicolumn{2}{c}{ Sowing } & \multicolumn{2}{c}{ Soil } & \multicolumn{2}{c}{ Sowing x Soil } \\
Year & Pseudo-F & p-value & Pseudo-F & p-value & Pseudo-F & p-value \\
\hline 1997 & 43.42 & $\mathbf{0 . 0 0 1}^{* * *}$ & 11.15 & $\mathbf{0 . 0 0 3}^{* *}$ & 3.36 & 0.060 \\
1998 & 32.97 & $\mathbf{0 . 0 0 1}^{* * *}$ & 6.33 & $\mathbf{0 . 0 0 7}^{* *}$ & 3.60 & 0.056 \\
1999 & 14.44 & $\mathbf{0 . 0 0 1}^{* * *}$ & 4.37 & $\mathbf{0 . 0 1 1}^{*}$ & 3.70 & $\mathbf{0 . 0 1 4}^{*}$ \\
2000 & 7.39 & $\mathbf{0 . 0 0 1}^{* * *}$ & 2.30 & 0.054 & 0.83 & 0.466 \\
2001 & 11.83 & $\mathbf{0 . 0 0 1}^{* * *}$ & 2.21 & 0.074 & 3.14 & $\mathbf{0 . 0 3 2}^{*}$ \\
2002 & 6.70 & $\mathbf{0 . 0 0 1}^{* * *}$ & 0.95 & 0.391 & 0.95 & 0.398 \\
2003 & 7.59 & $\mathbf{0 . 0 0 1}^{* * *}$ & 0.78 & 0.466 & 1.30 & 0.201 \\
2004 & 7.38 & $\mathbf{0 . 0 0 1}^{* * *}$ & 1.03 & 0.342 & 1.24 & 0.219 \\
2005 & 11.17 & $\mathbf{0 . 0 0 2}^{* *}$ & 0.98 & 0.336 & 0.84 & 0.427 \\
2006 & 6.82 & $\mathbf{0 . 0 0 1}^{* * *}$ & 0.48 & 0.877 & 1.40 & 0.165 \\
2007 & 6.97 & $\mathbf{0 . 0 0 1}^{* * *}$ & 0.62 & 0.683 & 0.57 & 0.756 \\
2008 & 7.76 & $\mathbf{0 . 0 0 1}^{* * *}$ & 1.02 & 0.246 & 0.30 & 0.909 \\
2009 & 10.48 & $\mathbf{0 . 0 0 1}^{* * *}$ & 0.65 & 0.636 & 0.93 & 0.382 \\
2010 & 6.49 & $\mathbf{0 . 0 0 1}^{* * *}$ & 0.63 & 0.562 & 0.23 & 0.912 \\
2011 & 12.66 & $\mathbf{0 . 0 0 1}^{* * *}$ & 0.69 & 0.466 & 0.46 & 0.656 \\
2012 & 11.61 & $\mathbf{0 . 0 0 1}^{* * *}$ & 0.74 & 0.459 & 0.71 & 0.510 \\
2013 & 8.72 & $\mathbf{0 . 0 0 1}^{* * *}$ & 0.65 & 0.549 & 0.03 & 0.985 \\
2014 & 9.06 & $\mathbf{0 . 0 0 1}^{* * *}$ & 0.67 & 0.536 & 1.03 & 0.347 \\
2015 & 8.74 & $\mathbf{0 . 0 0 1}^{* * *}$ & 1.30 & 0.225 & 1.14 & 0.320 \\
2016 & 7.43 & $\mathbf{0 . 0 0 1}^{* * *}$ & 1.95 & 0.085 & 0.92 & 0.376 \\
\hline & & & & & & \\
\hline
\end{tabular}


Table S4.3. Results of MRPP tests for differences in nematode community composition at family level per year.

\begin{tabular}{|c|c|c|c|c|c|c|}
\hline \multirow[b]{2}{*}{ Year } & \multicolumn{2}{|c|}{ Sowing } & \multicolumn{2}{|c|}{ Soil } & \multicolumn{2}{|c|}{ Sowing $\mathrm{x}$ Soil } \\
\hline & Pseudo-F & p-value & Pseudo-F & p-value & Pseudo-F & p-value \\
\hline 1998 & 5.78 & $0.001^{* * *}$ & 0.73 & 0.597 & 0.90 & 0.416 \\
\hline 1999 & 1.20 & 0.249 & 1.97 & 0.029* & 1.60 & 0.072 \\
\hline 2000 & 1.39 & 0.161 & 3.16 & $0.004^{* *}$ & 0.28 & 0.949 \\
\hline 2001 & 2.47 & $0.010^{* *}$ & 3.19 & $0.001^{* * *}$ & 0.46 & 0.889 \\
\hline 2002 & 2.14 & $0.045^{*}$ & 5.11 & $0.001^{* * *}$ & 1.11 & 0.342 \\
\hline 2003 & 2.70 & $0.009^{* *}$ & 5.65 & $0.001^{* * *}$ & 0.43 & 0.914 \\
\hline 2004 & 5.26 & $0.001^{* * *}$ & 6.67 & $0.001^{* * *}$ & 2.03 & 0.102 \\
\hline 2005 & 2.75 & $0.002^{* *}$ & 3.42 & $0.001^{* * *}$ & 1.09 & 0.396 \\
\hline 2006 & 2.22 & $0.005^{* *}$ & 1.85 & $0.019 *$ & 0.55 & 0.835 \\
\hline 2007 & 2.02 & $0.043^{*}$ & 3.43 & $\mathbf{0 . 0 0 3}^{* *}$ & 0.57 & 0.902 \\
\hline 2008 & 1.61 & 0.079 & 2.22 & $0.011^{*}$ & 0.82 & 0.620 \\
\hline 2009 & 1.92 & 0.052 & 3.16 & $\mathbf{0 . 0 0 3}^{* *}$ & 0.22 & 0.998 \\
\hline 2010 & 3.35 & $0.001^{* * *}$ & 1.09 & 0.306 & 1.91 & $0.025^{*}$ \\
\hline 2011 & 1.14 & 0.224 & 2.07 & $\mathbf{0 . 0 0 8}^{* *}$ & 0.60 & 0.763 \\
\hline 2012 & 3.26 & $0.001^{* * *}$ & 1.96 & 0.031* & 1.06 & 0.394 \\
\hline 2013 & 2.22 & $0.001^{* * *}$ & 1.31 & 0.112 & 1.01 & 0.258 \\
\hline
\end{tabular}


Table S4.4. Results of LMM analyses on similarity of the plant community composition to the donor community (recorded in 2011) per year.

\begin{tabular}{lcccccc}
\hline & \multicolumn{2}{c}{ Sowing } & \multicolumn{2}{c}{ Soil } & \multicolumn{2}{c}{ Sowing x Soil } \\
Year & $\mathrm{F}_{1,12}$ & $\mathrm{p}$-value & $\mathrm{F}_{1,12}$ & $\mathrm{p}$-value & $\mathrm{F}_{1,12}$ & p-value \\
\hline 1997 & 27.11 & $\mathbf{0 . 0 0 0 2}$ & 75.65 & $<\mathbf{0 . 0 0 0 1}$ & 3.98 & 0.069 \\
1998 & 2.93 & 0.113 & 0.09 & 0.772 & 0.47 & 0.507 \\
1999 & 15.72 & $\mathbf{0 . 0 0 1 9}$ & 0.83 & 0.380 & 2.32 & 0.153 \\
2000 & 21.44 & $\mathbf{0 . 0 0 0 6}$ & 3.52 & 0.085 & 0.57 & 0.465 \\
2001 & 8.97 & $\mathbf{0 . 0 1 1 2}$ & 0.32 & 0.584 & 8.32 & $\mathbf{0 . 0 1 4}$ \\
2002 & 3.92 & 0.0712 & 0.57 & 0.466 & 6.03 & $\mathbf{0 . 0 3 0}$ \\
2003 & 44.09 & $<\mathbf{0 . 0 0 0 1}$ & 0.99 & 0.339 & 2.05 & 0.178 \\
2004 & 14.20 & $\mathbf{0 . 0 0 2 7}$ & 1.10 & 0.315 & 4.61 & 0.053 \\
2005 & 16.79 & $\mathbf{0 . 0 0 1 5}$ & 0.64 & 0.439 & 0.99 & 0.340 \\
2006 & 16.68 & $\mathbf{0 . 0 0 1 5}$ & 0.02 & 0.901 & 1.12 & 0.312 \\
2007 & 14.50 & $\mathbf{0 . 0 0 2 5}$ & 0.60 & 0.456 & 0.11 & 0.751 \\
2008 & 8.21 & $\mathbf{0 . 0 1 4 2}$ & 0.50 & 0.494 & $<0.01$ & 0.967 \\
2009 & 21.05 & $\mathbf{0 . 0 0 0 6}$ & 0.21 & 0.654 & 0.20 & 0.663 \\
2010 & 2.88 & 0.116 & 0.02 & 0.897 & $<0.01$ & 0.971 \\
2011 & 1.11 & 0.313 & 0.05 & 0.834 & 0.10 & 0.755 \\
2012 & 9.66 & $\mathbf{0 . 0 0 9 0}$ & 0.33 & 0.579 & 0.01 & 0.910 \\
2013 & 10.66 & $\mathbf{0 . 0 0 6 8}$ & 0.12 & 0.732 & 1.05 & 0.325 \\
2014 & 4.78 & $\mathbf{0 . 0 4 9 4}$ & 0.01 & 0.934 & 1.16 & 0.302 \\
2015 & 6.90 & $\mathbf{0 . 0 2 2 1}$ & 0.12 & 0.732 & 0.31 & 0.586 \\
2016 & 7.77 & $\mathbf{0 . 0 1 6 4}$ & 0.05 & 0.82 & 0.07 & 0.803 \\
\hline & & & & & &
\end{tabular}


Table S4.5. Results of LMM analyses on similarity of the nematode community composition to the donor community (recorded in 2011) per year.

\begin{tabular}{lcccccc}
\hline & \multicolumn{2}{c}{ Sowing } & \multicolumn{2}{c}{ Soil } & \multicolumn{2}{c}{ Sowing x Soil } \\
Year & $\mathrm{F}_{1,12}$ & $\mathrm{p}$-value & $\mathrm{F}_{1,12}$ & $\mathrm{p}$-value & $\mathrm{F}_{1,12}$ & $\mathrm{p}$-value \\
\hline 1998 & 9.33 & $\mathbf{0 . 0 1 0}$ & 0.20 & 0.663 & 2.42 & 0.146 \\
1999 & 0.31 & 0.591 & 12.21 & $\mathbf{0 . 0 0 4 4}$ & 4.77 & $\mathbf{0 . 0 4 9 6}$ \\
2000 & 0.06 & 0.816 & 9.83 & $\mathbf{0 . 0 0 8 6}$ & 0.61 & 0.450 \\
2001 & 2.53 & 0.138 & 19.20 & $\mathbf{0 . 0 0 0 9}$ & 0.89 & 0.365 \\
2002 & 0.75 & 0.403 & 5.07 & $\mathbf{0 . 0 4 3 9}$ & 0.20 & 0.660 \\
2003 & 2.56 & 0.136 & 52.30 & $<\mathbf{0 . 0 0 0 1}$ & 0.28 & 0.609 \\
2004 & 1.59 & 0.232 & 6.88 & $\mathbf{0 . 0 2 2 3}$ & 1.39 & 0.262 \\
2005 & $<0.01$ & 0.963 & 8.01 & $\mathbf{0 . 0 1 5 2}$ & 6.84 & $\mathbf{0 . 0 2 2 6}$ \\
2006 & 11.89 & $\mathbf{0 . 0 0 4 8}$ & 11.35 & $\mathbf{0 . 0 0 5 6}$ & 0.09 & 0.769 \\
2007 & 2.74 & 0.124 & 3.83 & 0.074 & 2.49 & 0.141 \\
2008 & 0.10 & 0.764 & 1.68 & 0.220 & 0.10 & 0.761 \\
2009 & 3.74 & 0.077 & 1.61 & 0.229 & 2.44 & 0.144 \\
2010 & 14.48 & $\mathbf{0 . 0 0 2 5}$ & 7.60 & $\mathbf{0 . 0 1 7 4}$ & $<0.01$ & 0.988 \\
2011 & 2.32 & 0.154 & 5.23 & $\mathbf{0 . 0 4 1 1}$ & 1.93 & 0.190 \\
2012 & 7.52 & $\mathbf{0 . 0 1 7 8}$ & 0.96 & 0.348 & 0.01 & 0.942 \\
2013 & 3.48 & 0.087 & 5.57 & $\mathbf{0 . 0 3 6 0}$ & 3.95 & 0.070 \\
\hline
\end{tabular}




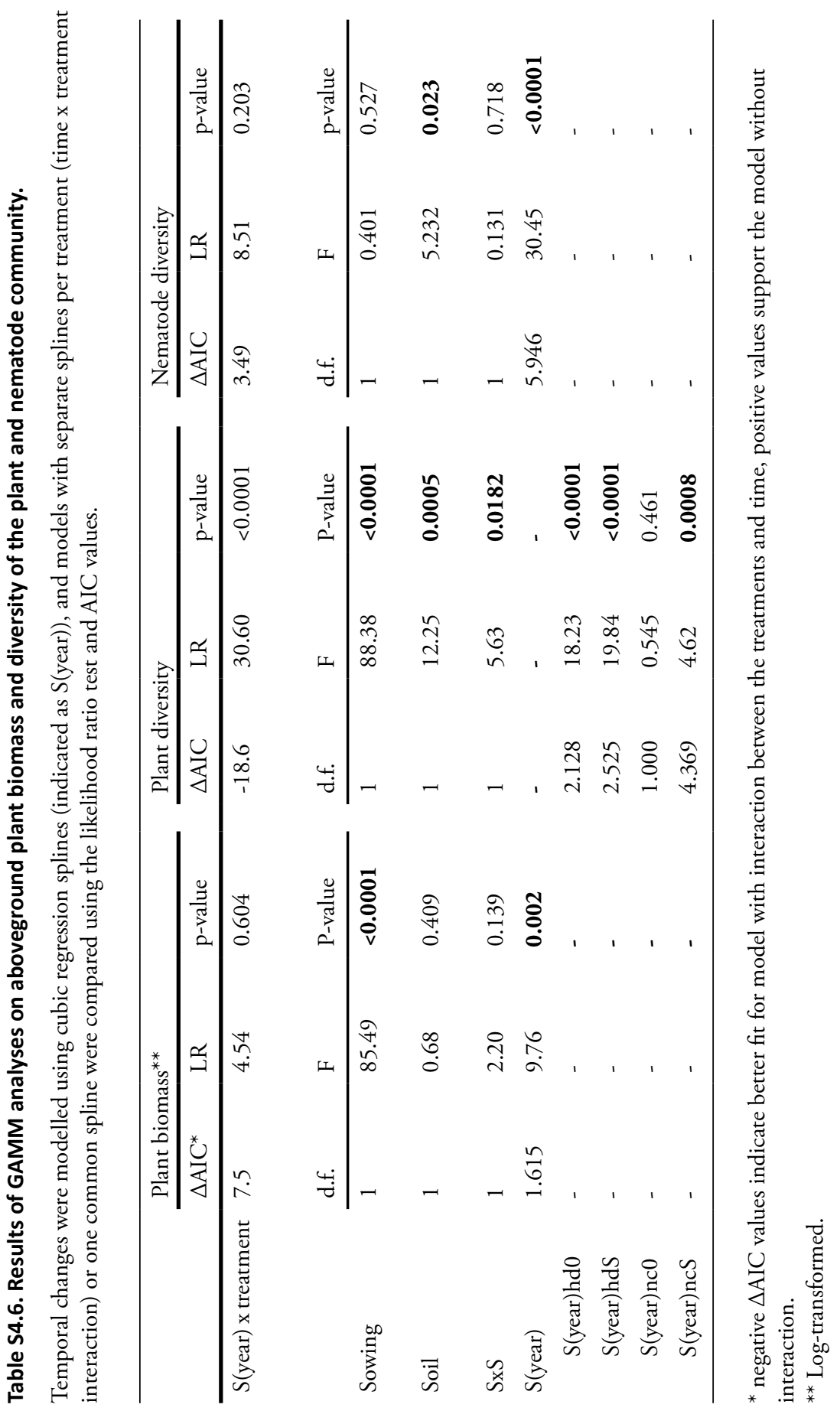



CHAPTER 5

Effects of spatial plant-soil feedback heterogeneity on plant performance in monocultures

E. R. Jasper Wubs and T. Martijn Bezemer

Journal of Ecology (2016) 104, 364-376 


\begin{abstract}
Plant-soil feedback (PSF) effects have almost exclusively been quantified on homogeneous soils, but as different plant species will influence their local soil differently PSF effects will in reality be spatially heterogeneous. Whether plant performance in soils with spatially heterogeneous PSF can be predicted from pot experiments with homogeneous soils is unclear.
\end{abstract}

5 In a greenhouse experiment we tested the response of monocultures of six grassland species (two grasses, two legumes, and two forbs) to three spatially explicit PSF treatments (fine-grain heterogeneity, coarse-grain heterogeneity, and homogeneous). Sixteen patches of conditioned soil $(-6 \times 6 \mathrm{~cm})$ were placed within each container. For homogeneous treatments all patches within a container contained the same conditioned soil. The fine-grained heterogeneous treatment contained four differently conditioned soils that were applied following a Latin square design, while for the coarse-grained heterogeneous treatment four contiguous square blocks of four cells each were created in each container.

In general species grew worse on soil conditioned by conspecifics. However, when the biomass production on all homogeneous soil treatments (own and foreign soils) was averaged and compared to the heterogeneous treatments, we found that biomass production was lower than expected in the heterogeneous soils. This effect of heterogeneity depended on both the conditioning and test species, but most heterogeneity effects were negative. The grain of the heterogeneity (coarse vs. fine: at the chosen spatial scale) did not affect plant performance.

We hypothesize that a more diverse soil community is present in spatially heterogeneous soils. This i) increases the chance of plants to encounter its antagonists, which may then rapidly increase in numbers; and ii) widens the scope for synergistic co-infections. Together this may lead to non-additive responses of plants to spatial PSF heterogeneity.

Plant performance was lower in spatially heterogeneous soils than predicted by spatially homogeneous soils. In natural grasslands that have mixed plant communities conditioning the plant-soil feedback (PSF) effects on plant performance may therefore be more negative than what is predicted from pot experiments. Our results emphasise the need to incorporate the spatial dynamics of PSF both in empirical and modelling studies if we are to understand the role of PSF in plant-plant interactions and plant community dynamics. 


\section{Introduction}

A rapidly increasing number of studies have argued that plant-soil feedbacks (PSF) may play a profound role in determining plant performance and the composition of natural vegetation (Van der Putten et al. 2013). Mathematical models have shown that spatial heterogeneity in PSF effects at the plot level can greatly influence plant community composition (Bonanomi et al. 2005, Eppinga et al. 2006, Mack and Bever 2014). However, so far empirical PSF studies have almost exclusively been conducted in spatially homogeneous soils and whether those empirical PSF data can reliably be used to extrapolate to spatially heterogeneous conditions is an open question.

Spatial heterogeneity has long been investigated as a driver of plant community composition (Reynolds and Haubensak 2009, Lundholm 2009, Tamme et al. 2010, Allouche et al. 2012), but this work has almost completely been restricted to heterogeneity in abiotic conditions (e.g. acidity, water table, nutrient availability and form). However, it is becoming increasingly clear that at small scales (Bezemer et al. 2010) spatial heterogeneity effects may largely be driven by biotic interactions and this may strongly affect plant-plant interactions (Tamme et al. 2010, De Kroon et al. 2012). The biotic drivers could be for example localized plant-microbe interactions that affect nutrient mineralization or accumulation of pathogens.

Plant-soil feedback studies assess the consequences of plant induced changes in the soil's biotic as well as abiotic conditions for plant performance (Bever 1994, Bardgett et al. 1999, Ehrenfeld et al. 2005, Casper and Castelli 2007, Van der Putten et al. 2013). Soil microbial communities change in response to differences in root-associated rhizodeposits among plants species (Raaijmakers et al. 2009, Bever et al. 2012, Philippot et al. 2013). These rhizodeposits include carbon-sources as well as secondary compounds involved in plant defence that differentially affects the population growth rates of different microbes. The effect of soil conditioning on plant performance, i.e. the feedback, can be positive and negative depending on both conditioning and responding plant species (Bever et al. 1997, Bever 2003, Van der Putten et al. 2013). Furthermore, PSF can arise from conditioning by conspecific individuals (direct or conspecific PSF) or heterospecific individuals (indirect or heterospecific feedback; Bever et al. 1997, Van de Voorde et al. 2011). Most direct (conspecific) plant-soil feedbacks are negative (Petermann et al. 2008, Kulmatiski et al. 2008).

The composition of the soil community is strongly heterogeneous in space across a range of scales (Ettema and Wardle 2002, Bever et al. 2010), from changes 
in rhizosphere communities along individual roots (Folman et al. 2001) up to macroecological patterns in species assemblages (Green and Bohannan 2006). At relatively small scales individual plants can alter the composition of the soil community (Bever 1994, Grayston et al. 1998, Bever et al. 2010, Van der Putten et al. 2013). As different plant species induce different rhizosphere conditions they promote different soil communities and this leads to a patchy below-ground 'mosaic' of soil communities (Bever et al. 2010). In line with this, distinct soil communities under different plant species have been observed at the level of individual plants ( $5 \mathrm{~cm}$ diameter soil cores) in diverse plant communities in the field (Bezemer et al. 2010) and the density of conspecifics in the field affects the feedback strength (Kos et al. 2013). However, besides the identity of the conditioning plant, the composition of the local soil community is also to some extent influenced by the composition of the surrounding plant community (Bezemer et al. 2010). Consequently, while the mosaic structure of plant species specific conditioning effects can clearly be observed belowground in field, in reality also neighbouring plants contribute to local soil conditioning as a plant's zone of influence typically extends beyond neighbouring plants (Casper et al. 2003). We use the term spatial PSF heterogeneity here to indicate that adjacent patches differ in their feedback effects and we contrast this with homogeneous soil that consists of a larger patch conditioned uniformly by one species.

To date the vast majority of studies of the consequences of PSFs for plant communities have relied on homogeneous soils. However, a few recent experiments have directly addressing PSF in spatially explicit settings. These studies have shown that small scale PSF heterogeneity can alter plant vital rates and affect rates of invasion by competitors (Brandt et al. 2013, Burns and Brandt 2014). Furthermore, analogous to root foraging for nutrients, plants also respond to spatial differences in soil community composition by selectively placing their roots in patches conditioned by heterospecifics ('foreign' soil) over patches conditioned by conspecifics ('own' soil; Hendriks et al. 2015b).

While these studies established that small-scale spatial PSF heterogeneity can affect plant performance, it is still unclear whether spatially heterogeneous PSF effects are predictable from pot experiments with spatially homogeneous soils. This is a critical next step in upscaling the role of individual plant-soil interactions to whole plant communities in field settings (Kardol et al. 2013, Hawkes et al. 2013). In addition, recent modelling work has shown that the spatial scale over which PSF affects plants (feedback neighbourhood size) can significantly influence 
the consequences of PSF for plant communities (Mack and Bever 2014), but this has not been tested experimentally.

Here we test explicitly if plant performance on soils with spatial heterogeneous PSF effects can be predicted from performance on homogeneous soils. In addition, we test for the first time how the grain of spatial PSF heterogeneity influences plant growth. We quantified plant performance of six focal species grown in monoculture on soils conditioned by these species. The conditioned soils were placed in three spatial PSF treatments (fine-grain heterogeneity, coarse-grain heterogeneity, and homogeneous; Fig. 5.1). In each treatment containers were filled with 16 patches of conditioned soil. Spatial PSF heterogeneity was introduced by placing multiple differently conditioned soil patches within a single container. In the heterogeneous treatments the local soil patch quality (texture, nutrient availability, soil biotic

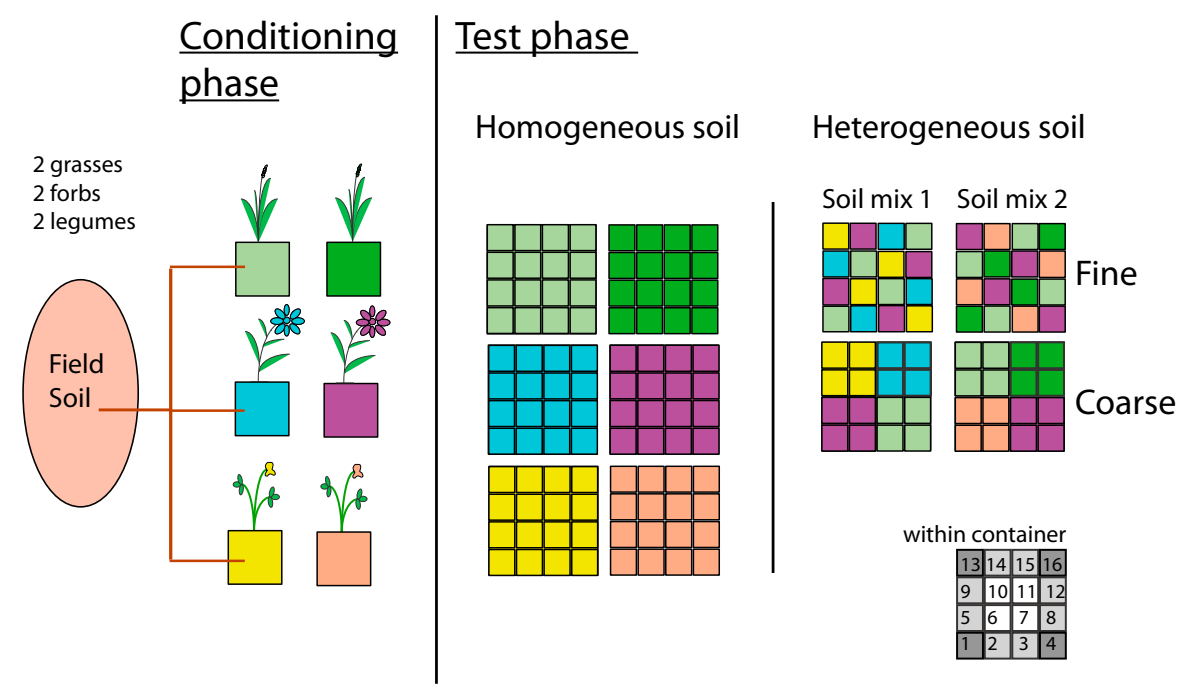

Fig. 5.1. Conceptual representation of the experimental design.

Common field soil was conditioned by monocultures of six plant species. The six soil conditioning treatments are identified by having the colour of the soils (squares) corresponding to the colours of the flowers. Three independent soil replicates were made for each of the conditioned soils. The conditioned soils were used to create spatially homogeneous as well as heterogeneous (coarse and fine) soil treatments in the test phase. The heterogeneous treatments were created using two soil combinations, each containing soil conditioned by four species. Each of the ten soil treatments was planted with each of the six focal species in monoculture full factorially in the test phase. In the inset the numbering of the gridcells is shown, the different shadings show how the within container design has rotational symmetry. For further details see main text. 
community) was the same as in the homogeneous treatments as the same soil was used to create the soil patches (i.e. no conditioned soils were mixed) - only their spatial arrangement differed. Our null-model was that the performance of a plant on a local patch of soil in a heterogeneous set of patches would be identical to its performance on the same soil in a homogeneous set of patches. Hence we expected that overall performance of a plant monoculture in spatially heterogeneous PSF soils would be isometrically (1:1) predictable as the mean performance of that plant on each of the constituent soils in homogeneous conditions (when the calculation is weighted by the proportion of each conditioned soil type in the heterogeneous soil). This null-model assumes that all plant-soil interactions are local (i.e. within a patch) and that the soil communities in the differently conditioned soils have no significant interactions that affect plant performance. These are assumptions commonly made in models of PSF (e.g. Bonanomi et al. 2005, Eppinga et al. 2006). Furthermore, we expected that if spatial heterogeneity alters the effects of PSFs, the difference would be most pronounced in containers with fine scale heterogeneity, while containers with coarse scale heterogeneity would be intermediate relative to spatially homogeneous soil. Finally, we expected that the strength of direct PSF ('own' vs. 'foreign' comparison) would become less strong as the grain of heterogeneity becomes smaller (PSF: homogeneous $>$ coarse$>$ fine-grained heterogeneity).

\section{Material \& Methods}

We conducted a plant-soil feedback greenhouse experiment in which we grew six focal plant species on six conditioned soils and where we explicitly manipulated the spatial heterogeneity of conditioned soils in the containers (Fig. 5.1). Six plant species were selected that are typical for old-fields on sandy soils in northwest Europe, with two representatives each for grass, forb and legume functional groups. They were respectively: Agrostis capillaris L. and Festuca rubra L., Hypochaeris radicata L. and Jacobaea vulgaris Gaertn. (syn. Senecio jacobaea L.), and Lotus corniculatus L. and Trifolium pratense L. Plant-soil feedback experiments typically consist of two phases, first one where plants condition the soil (conditioning phase) and subsequently a phase where the effects of the soil conditioning on plant growth are tested (test or feedback phase).

\section{Phase 1: Conditioning phase}

Soil was collected from a grazed old-field restoration grassland (Mossel, Planken Wambuis, Ede, the Netherlands, GPS: $52^{\circ} 04^{\prime} \mathrm{N} 05^{\circ} 45^{\prime} \mathrm{E}$ ) where agricultural practices were ceased in 1995, in September 2012. In total approximately 2,500 
$\mathrm{kg}$ soil was collected from the topsoil (to $30 \mathrm{~cm}$ depth) and sieved over a $5 \mathrm{~mm}$ mesh. The soil was used to fill large square containers (length $\mathrm{x}$ width $\mathrm{x}$ height: $17 \times 17 \times 17 \mathrm{~cm}$ ) with $5 \mathrm{~kg}$ soil per container (Fig. 5.1). Seeds for each of the species were obtained from a specialized company that provides seeds from wild plants (Cruydt-hoeck, Assen, the Netherlands) or collected from the same field as the soil (J. vulgaris). All seeds were surface-sterilized ( $1 \mathrm{~min}$. in $<2.5 \% \mathrm{NaClO}$ solution), rinsed with water and allowed to germinate on sterilized glass beads in a climate chamber (16:8h day-night cycle, continuous $20^{\circ} \mathrm{C}$ ) for two weeks. Seedlings of each of the six species were planted in monocultures (16 individuals per container) creating 58 containers per species, except for $A$. capillaris and J. vulgaris with 77 containers each. More soil of the latter two species was needed to create the spatially heterogeneous treatments in the test phase (see below). All containers were randomly located within a greenhouse compartment, but each container was a priori allocated to one of three replicates and these replicates were maintained throughout the experiment. The plants were allowed to grow in the greenhouse (16:8h day:night, natural light supplemented with $600 \mathrm{~W}$ metal-halide lamps, 1 per $4 \mathrm{~m}^{-2}$, approx. $225 \mu \mathrm{mol}$ light quanta $\mathrm{m}^{-2} \mathrm{~s}^{-1}$ at plant level, $21: 16^{\circ} \mathrm{C}$ day:night, $50-70 \%$ relative humidity) for 8 weeks. Subsequently all above-ground biomass was clipped flush with the soil, dried $\left(72{ }^{\circ} \mathrm{C}, 48 \mathrm{~h}\right)$ and weighed. We created three independent soil replicates for each of the six conditioning species by pooling and homogenizing the soil only from those containers that were a priori allocated to the same replicate. During homogenization all root systems were removed. To obtain a sufficient amount of soil for the test phase each of the 18 soil replicates ( 6 conditioned soils $\mathrm{x} 3$ replicates) was mixed with sterilized (>25KGray gamma radiation, Isotron, Ede, the Netherlands) field soil collected from the same site in a 8.4:1.6 (conditioned:sterile w:w) ratio. From each of the homogenized soil replicates a sample $(200 \mathrm{~g})$ was taken for chemical analysis upon addition of the sterilized soil. We measured $\mathrm{NH}_{4}, \mathrm{NO}_{3}$ (both KCl-extraction), $\mathrm{PO}_{4}$ (P-Olsen extraction) and soil organic matter (ashed at $430{ }^{\circ} \mathrm{C}$ for $24 \mathrm{~h}$ ) content as well as soil acidity (in 1:2.5 w:w dry soil : water suspensions; see Table S5.1).

\section{Phase 2: Test phase}

In the test phase three different levels of spatial PSF heterogeneity were created (spatially homogeneous, spatially heterogeneous coarse-grained, and spatially heterogeneous fine-grained) each applied to different large containers (Fig. 5.1). Each container (length $\times$ width $\times$ height: $26 \times 22 \times 22 \mathrm{~cm}$ ) was divided with a custom made metal grid into $4 \times 4$ cells, each with a surface area of $-35 \mathrm{~cm}^{2}$ (the length and width of the cells differed slightly to account for the rounded 
corners of the containers), extending to the bottom of the container. The size of the gridcells was chosen because at this grain size systematic differences in soil community composition were detected in open communities in the field (Bezemer et al. 2010). In each container, independent of the treatment, all 16 gridcells were filled individually and any given gridcell was always filled with soil conditioned by a single species. Immediately after filling the containers the grids were removed so that during the experiment the soil patches in each container were in full contact. One of the corners of each of the containers was marked as a point of reference. For the spatially homogeneous treatment all cells in a container were filled with soil conditioned by the same species, while for spatially heterogeneous treatments (coarse- and fine-grained) gridcells were filled with soil conditioned by four different species (Fig. 5.1). The four soils in the fine-grained treatment were applied following a Latin square design, while for the coarse-grained treatment four contiguous square blocks of four cells each were created in each container. The two spatially heterogeneous treatments (coarse- and fine-grained) were created with two different mixes of soil conditioned by four species (soil mix). Soil mix 1 consisted of soils conditioned by $A$. capillaris, J. vulgaris, $H$. radicata and L. corniculatus; Soil mix 2 consisted of A. capillaris, J. vulgaris, F. rubra, and T. pratense. Consequently, both soil mixes had at least one representative each of the grass, forb and legume plant functional types. Soils from all six focal species were used separately to create spatially homogeneous containers. Subsequently, each of the ten soil-by-spatial heterogeneity treatments ( 6 homogeneous, 2 coarse and 2 fine) was planted with monocultures of each of the six test species. The whole setup was replicated three times, using the three independent soil replicates. In total there were 180 containers in the test phase ( 10 soil treatments $\mathrm{x}$ six test plant species $\mathrm{x}$ three replicates).

Each container was filled with $2.5 \mathrm{~kg}$ sterilized gravel (quartz, 4-8 $\mathrm{mm}$ ) and then with $8 \mathrm{~kg}$ of conditioned soil $(500 \mathrm{~g}$ per gridcell). For each treatment the containers were filled with conditioned soil in the same way: weighing 500 $\mathrm{g}$ of the appropriate conditioned soil type and then carefully pouring the soil into the respective gridcell and continuing until all cells of the container were filled. Each container was planted with 32 seedlings, planting two individuals into each gridcell (each seedling $1 \mathrm{~cm}$ from the gridcell midpoint). Seeds were germinated in the same way as in the conditioning phase. Seedlings that died upon transplantation were replaced once during the first week. The containers were placed in the greenhouse in a complete randomized design under the same conditions as during the conditioning phase and allowed to grow for eight weeks. 
The soil was kept moist by regular watering ( 2 or 3 times per week depending on evapotranspiration rates). After 8 weeks of growth, above-ground plant biomass was clipped flush with the soil, dried $\left(72{ }^{\circ} \mathrm{C}, 48 \mathrm{~h}\right)$, and weighed separately per gridcell for each of the containers (i.e. 16 observations per container, with known locations of each observation within the container). Below-ground biomass was sampled by inserting a soil corer $(\varnothing 3.3 \mathrm{~cm})$ into the middle of a gridcell and gently pushing it to the bottom of the container. While extracting the corer it was made sure that all soil in the core down to the gravel underneath, was collected. To make sure the soil cores were taken exactly in the middle of each gridcell a metal grid (same dimensions as before, but only $1 \mathrm{~cm}$ high) was placed on top of the soil when taking soil cores. Roots were extracted from the soil cores by careful washing over a sieve ( $2 \mathrm{~mm}$ mesh) and subsequently dried and weighed. For the spatially heterogeneous treatments (coarse- and fine-grained) all sixteen gridcells were sampled while from for the spatially homogeneous treatment eight cores were taken (cell numbers 2, 4, 5, 7, 10, 12, 13, and 15, Fig. 5.1).

\section{Data analysis}

Differences in abiotic conditions and shoot biomass at the end of the conditioning phase were tested with one-way ANOVA's. Spearman correlations were used to assess the relationship of the abiotic soil variables at the end of the conditioning phase and the above- and below-ground biomass of each of the test species at the end of the test phase. Because each test species was grown on the same set of soils ( 6 conditioned soils $* 3$ soil replicates) these correlations were calculated for each test species separately. Plant biomass data from the test phase were analysed at container level and at gridcell level separately to study overall effects of changing spatial heterogeneity and specific responses to differently conditioned soils in detail.

Container level: Above-ground biomass at container level was calculated as the sum of the biomass of all sixteen gridcells. As below-ground biomass was determined in soil cores and not in the entire soil volume, it was analysed as mean biomass per soil core. To test for the effect of spatial PSF heterogeneity on plant performance we compared plant biomass across the three levels of spatial heterogeneity. Containers in each heterogeneous treatment contained four soils, while in the homogeneous treatment they contained only one. To facilitate comparison we calculated an expected plant biomass from the same four soils in the homogeneous treatment assuming, as a null-expectation, that there was no additional effect of spatial PSF heterogeneity. Therefore, we calculated the null-expectation as the mean performance of the test species in spatially homogeneous containers 
on the same four soils that constituted the heterogeneous treatment. The nullexpectation was calculated separately for both soil mixes and each independent soil replicate. The calculated expected biomass values were used in an analysis to act as the control treatment (i.e. reflecting plant performance on the constituent soils if no spatial interaction were to occur). The biomass in the heterogeneous treatments was included in the analyses as it was observed. Consequently, the analyses were based on 108 data points: 6 test species $\mathrm{x} 2$ soil mixes x 3 spatial treatments (fine-, coarse-grain heterogeneous and expected-from-homogeneous) $x 3$ replicates. The effect of spatial heterogeneity on container level was analysed in separate general linear models (GLM) for above- and below-ground biomass. Test plant species, soil mix and spatial heterogeneity treatment (fine, coarse and expected-from-homogeneous) and their interactions were included as fixed effects. Post-hoc comparisons among levels of spatial heterogeneity were tested as planned contrasts both overall across all six test species as well as for each test species separately (Crawley 2002, Brinkman et al. 2010, Galecki and Burzykowski 2013).

To determine how soil conditioning affected plant growth (plant-soil feedback), we analysed data from the spatially homogeneous containers separately. Whole-container above- and below-ground biomass was analysed using two-way ANOVA's (6 soils $\mathrm{x}$ 6 species $\times 3$ replicates $=108$ containers), with both conditioned soil type and test plant species as fixed effects. Within the overall model the effect of 'own' relative to 'foreign' soil was analysed using planned contrasts for each test species separately.

Gridcell level: We analysed the above- and below-ground biomass on gridcell level using Linear Mixed Models (LMMs). Test plant species, conditioned soil type and spatial PSF heterogeneity treatment (fine-, coarse-grained heterogeneity, and homogeneous) and their interactions were included as fixed effects. The models included nested random effects for soil replicates, container, and gridcell. The gridcell factor was introduced to account for positional effect within containers, but given the rotational symmetry in the within container design the gridcell factor had three levels (corner (4 per container), edge (8), or centre (4) of the container; Fig. 5.1). Analyses with a 16-level random effect for gridcell lead to the same qualitative conclusions (c.f. Table $5.2 \&$ S5.2). The above-ground analysis was based on 2880 data points: 6 test species $\times 10$ soil treatments ( 6 homogeneous, 2 coarse-scale heterogeneous treatments and 2 fine-scale heterogeneous treatments) $\times 3$ replicates x 16 observations per container. For root biomass there were 2016 data points as in the six homogeneous soils only eight instead of 16 gridcells were sampled. 
Differences among levels of spatial heterogeneity were tested as planned contrasts both overall as well as for each test species on each conditioned soil separately.

Plant-soil feedback has often been characterized as the difference in plant performance on self-conditioned or 'own' soils versus the performance on soil conditioned by heterospecifics or 'foreign' soil (direct PSF; Van der Putten et al. 2013). To test if the direct PSF changed in response to different levels of spatial heterogeneity we calculated the feedback as the log-ratio of plant biomass on own and foreign soils (Brinkman et al. 2010) per test species in each of the three different heterogeneity treatments. This PSF index was calculated separately for each soil replicate (i.e. $\mathrm{N}=6$ test species $\mathrm{x} 3$ spatial heterogeneity treatments $\mathrm{x} 3$ soil replicates $=54$ ). The feedback values were analysed using a two-way linear model with fixed effects for test plant species and spatial heterogeneity. Differences in PSF among spatial heterogeneity treatments were tested using planned contrasts. This was done for each test species separately.

In a number of gridcells some of the plants had died during the experiment. Mortality was treated as a binary variable which takes the value one when either one or both of the plants of a given gridcell had died. Seedling mortality was analysed at the gridcell level using a Generalized Linear Mixed Model (GLMM) with the same fixed and random effects as the LMM on shoot biomass above but with a binomial error distribution. The GLMM was fitted by maximum likelihood and the significance of model terms was evaluated using likelihoodratio tests. In addition, the LMMs on plant biomass were refitted and evaluated using only those gridcells where both seedlings survived until harvest. Finally, to check that plant rooting patterns were consistent across the spatial heterogeneity treatments we regressed belowground biomass on shoot biomass across all treatment combinations at container level. We compared the fit to a model with three separate slopes for each of the three treatments by means of an F-test for coincidental regressions (Zar 1999 p. 375).

All analyses were conducted in $\mathrm{R}$ version 2.15.3 ( $\mathrm{R}$ Core Team 2016). Linear (mixed) models were fitted with the nlme (version 3.1-108; Pinheiro et al. 2016), GLMMs in lme4 (v1.0-4; Bates et al. 2013) R-packages. All models were graphically checked for homogeneity of variances and their error distributions. 


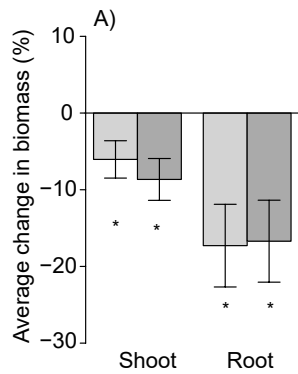

B) $\square$ Expected from homogeneous
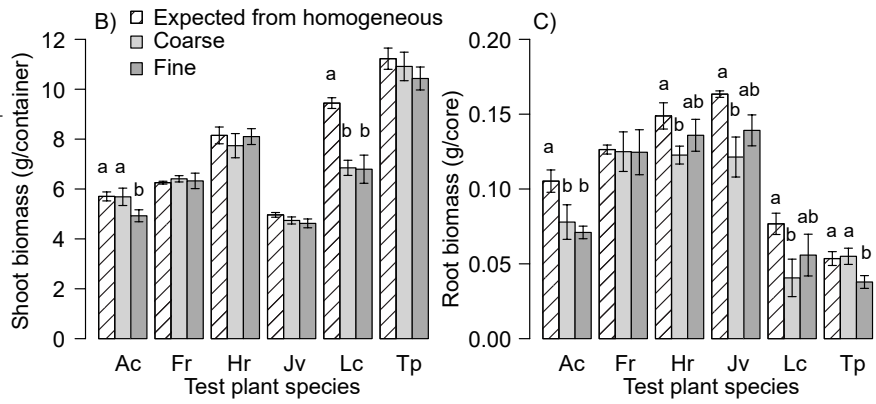

Fig. 5.2. Effect of different levels of spatial PSF heterogeneity (container level) on plant performance.

A) The effect of spatial PSF heterogeneity on plant performance averaged over the test species. Presented is the change in biomass relative to the expected biomass calculated from spatially homogeneous soils. The effects of both coarse (light grey) and fine (dark grey) scale heterogeneity are shown. B and C) Mean shoot (B) and root (C) biomass for each of six different test species in the three levels of spatial PSF heterogeneity. Observed biomass is given for coarse and fine spatial treatments, while the calculated expected biomass based on plant performance in spatially homogeneous soils is given for reference (indicated by the hatched bars). In both spatially heterogeneous treatments (coarse and fine) the test plants grew on soil patches conditioned by four species (c.f. Fig. 5.1). Plant performance on the same four soils in the spatially homogeneous treatment was used to calculate the expected plant performance in the heterogeneous treatments (see Methods for details). Stars indicate significant differences from zero (A; see Table 5.1 for contrast results) and different letters above the bars (B \& C) indicate significant differences in biomass among spatial PSF heterogeneity treatments within each test species (i.e. no among species comparisons were made; for the overall analyses see Table 5.1). Ac: Agrostis capillaris, Fr: Festuca rubra, Hr: Hypochaeris radicata, Jv: Jacobaea vulgaris, Lc: Lotus corniculatus, Tp: Trifolium pratense.

When the multi-species (test species) models were heteroscedastic, separate variances per test species were included in the models using generalized least squares (Pinheiro and Bates 2000, Zuur et al. 2009). Post-hoc comparisons and planned contrasts were made using the multcomp (v1.2-18) package in R (Hothorn et al. 2008). Posthoc tests were corrected using the method of Benjamini and Hochberg (1995).

\section{Results}

\section{Effect of spatial PSF heterogeneity on plant performance - container level}

Spatial PSF heterogeneity significantly affected plant performance (Table 5.1), which was reduced both in fine- and coarse-grained heterogeneity treatments relative to the performance on spatially homogeneous soils (Fig. 5.2A). On average, plants produced less above- $(7.4 \%)$ and belowground $(17.0 \%)$ biomass in 
Table 5.1. Analysis of variance (SS type I) table of GLMs on plant shoot and root biomass at the container level in the spatial PSF heterogeneity experiment.

Presented are degrees of freedom (d.f.), F-values, Z-values for overall planned contrasts among spatial PSF heterogeneity treatments, and p-values.

\begin{tabular}{lccccccccc}
\hline & & \multicolumn{3}{c}{ Shoot biomass } & & \multicolumn{3}{c}{ Root biomass } \\
Term/contrast & d.f. & F & Z & p-value & & F & Z & p-value \\
\hline Plant species (PS) & 5 & 130.42 & - & $<0.0001$ & & 83.66 & - & $<0.0001$ \\
Soil mix (SM) & 1 & 1.87 & - & 0.18 & & 1.47 & - & 0.23 \\
Spatial heterogeneity (SH) & 2 & 5.67 & - & 0.006 & & 9.32 & - & 0.0003 \\
$\quad$ Coarse vs. Homogeneous & & - & -2.86 & 0.004 & & - & -.4 .15 & $<0.0001$ \\
$\quad$ Fine vs. Homogeneous & & - & -3.18 & 0.0001 & & - & -3.46 & 0.0005 \\
$\quad$ Coarse vs. Fine & & - & -0.95 & 0.34 & & 0.69 & 0.49 \\
PS x SM & 5 & 0.92 & - & 0.48 & & 2.49 & - & 0.04 \\
PS x SH & 10 & 2.70 & - & 0.006 & & 1.76 & - & 0.08 \\
SM x SH & 2 & 0.23 & - & 0.79 & & 0.88 & - & 0.42 \\
PS x SM x SH & 10 & 0.77 & - & 0.66 & & 0.93 & - & 0.51 \\
Residual & 72 & & & & & & \\
\hline
\end{tabular}

spatially heterogeneous conditions than predicted from their performance on the same soils in homogeneous conditions (Table 5.1; Fig. 5.2A). There was however variation in the strength of the response of test species to PSF heterogeneity (Table 5.1). In terms of aboveground biomass, L. corniculatus and A. capillaris experienced significant reductions in the spatially heterogeneous treatments, while for the other species there was no strong effect (Fig. 5.2B). Below-ground, the effect of spatial heterogeneity was more general, only the root biomass of $F$. rubra was not significantly affected by spatial heterogeneity (Fig. 5.2C). For both above- and belowground biomass, however, the grain (patch size) of spatial PSF heterogeneity (coarse vs. fine) did not have a significant effect (Fig. 5.2A; Table 5.1). Regressions of root biomass as a function of shoot biomass for each of the test species showed that the model fit was not improved significantly by including separate slopes for each of the three levels of spatial heterogeneity (Table S5.3, Fig. S5.1). In addition, the confidence intervals of the regression slopes for all three spatial heterogeneity treatments overlap for each of the test species. This indicates that the relationship between root and shoot biomass was consistent across the treatments. Shoot biomass was overall a significant predictor of root biomass for most species (Table S5.3). For the two species where this was not the case the 

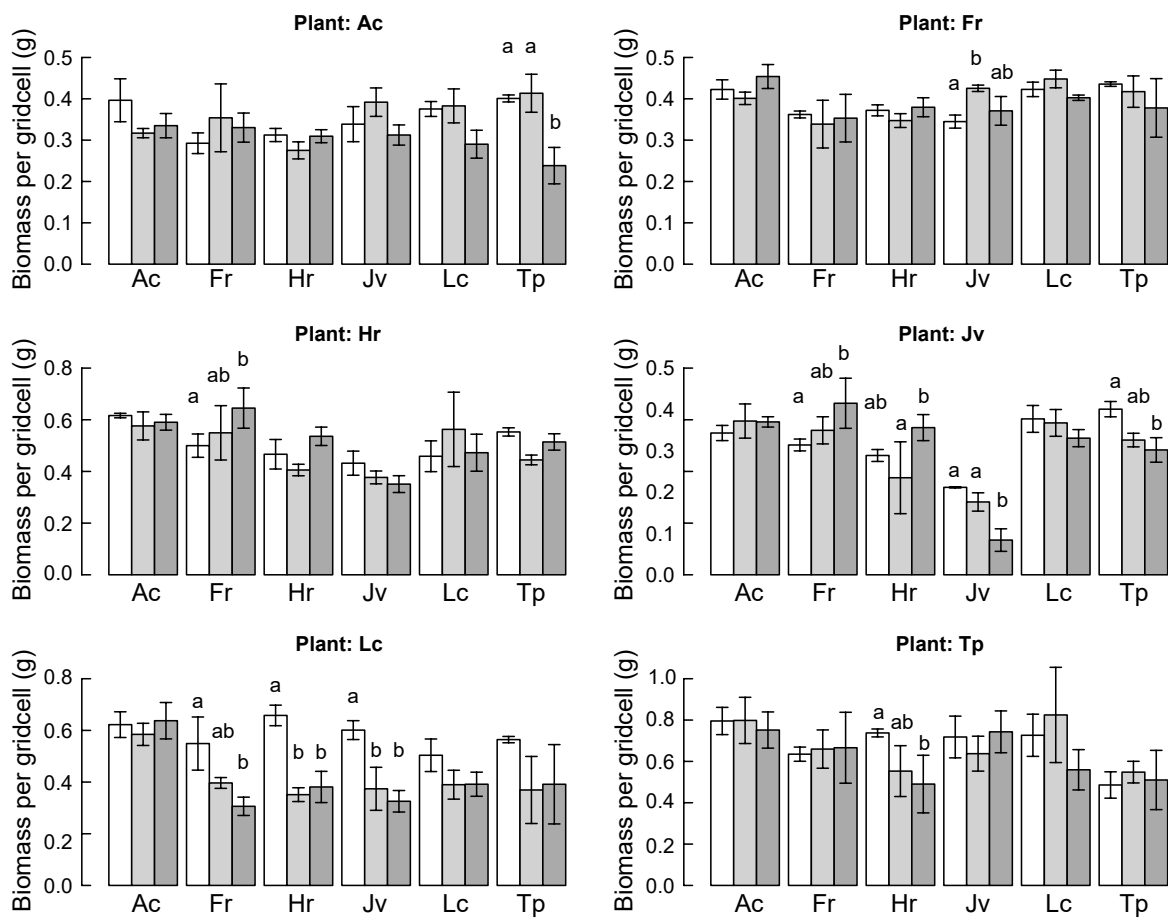

Soil conditioning species

Fig. 5.3. Aboveground biomass per gridcell (mean $\pm S E$ ) for each test plant species on each of the six conditioned soils in three levels of spatial PSF heterogeneity (homogeneous: white bars, coarse-grain: light grey bars, and fine-grain: dark grey bars).

Different letters above bars indicate significant differences among treatments within each test species and conditioned soil - based on gridcell level mixed model analyses (Table 5.2). Abbreviations as in Fig. 5.2.

variation in shoot biomass was very small, making it hard to detect a relationship. The regression slopes were, however, positive also in these cases (Fig. S5.1).

\section{Effect of spatial PSF heterogeneity on plant performance - gridcell level}

Also at the level of individual gridcells above- and below-ground plant biomass were generally lower in the heterogeneous than the homogeneous treatments (respectively: -8.0 and $-16.4 \%$; Table 5.2). However, the effect of spatial heterogeneity differed between the test plant species and depended on the soil it was growing in (plant $x$ soil interaction; Table 5.2; Fig. 5.3 and 5.4). While the majority of heterogeneity effects were negative, we also observed some cases of positive influence (Fig. 5.3 and 5.4). These were however only significant in 

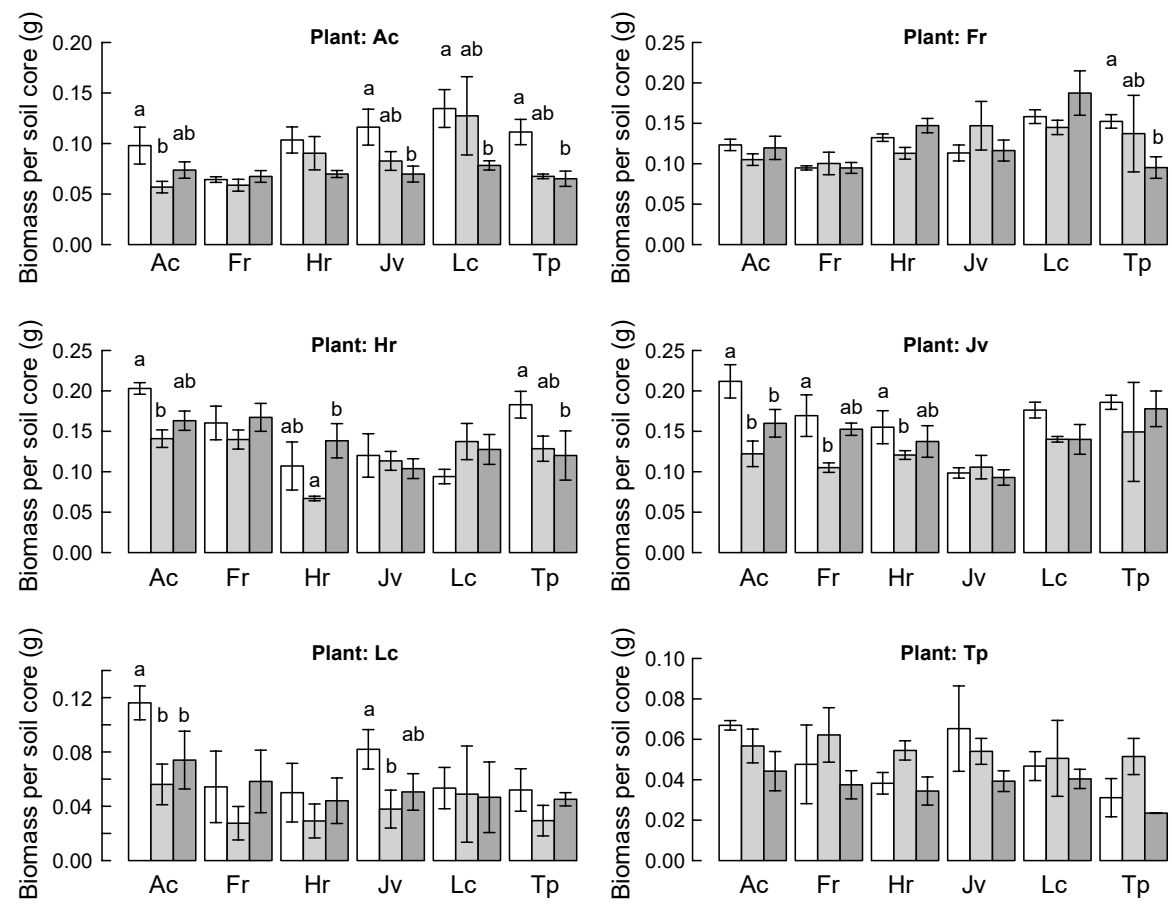

Soil conditioning species

Fig. 5.4. Belowground biomass (mean $\pm S E$ ) per soil core for each test plant species on each of the six conditioned soils in three levels of spatial PSF heterogeneity (homogeneous white bars, coarse-grain: light grey bars, and fine-grain: dark grey bars).

Different letters above bars indicate significant differences among treatments within each test species and conditioned soil - based on gridcell level mixed model analyses (Table 5.2). Abbreviations as in Fig. 5.2.

three cases (shoot biomass in combinations: plant $\mathrm{x}$ soil: F. rubra $\mathrm{x} J$. vulgaris, $H$. radicata $\mathrm{x} F$. rubra and J. vulgaris $\mathrm{x} F$. rubra; Fig. 5.3), indicating that negative heterogeneity effects dominated. Contrary to our expectation, the spatial grain of the heterogeneity did not significantly affect the magnitude of the heterogeneity effect (Table 5.2, Figs 5.3 and 5.4).

Plant mortality differed among test plant species and was affected by soil conditioning (Fig. S5.2; Table S5.4). There was a weakly significant interaction between test plant species and spatial heterogeneity $(P=0.04$; Table S5.4), but no consistent pattern across the test species. Mortality rates were generally low 
Table 5.2. Analysis of variance (SS type I) table of mixed models of plant biomass (shoots and root separately) in the spatial PSF experiment at gridcell level.

The models include nested random effects for soil replicate, container, and gridcell. Presented are degrees of freedom (d.f.), F-values, Z-values for overall planned contrasts among spatial PSF heterogeneity treatments, and p-values.

\begin{tabular}{|c|c|c|c|c|c|c|c|}
\hline \multirow[b]{2}{*}{ Term/contrast } & \multirow[b]{2}{*}{ d.f. } & \multicolumn{3}{|c|}{ Shoot biomass } & \multicolumn{3}{|c|}{ Root biomass } \\
\hline & & $\mathrm{F}$ & Z & p-value & $\mathrm{F}$ & Z & p-value \\
\hline Plant species (PS) & 5,276 & 134.62 & - & $<0.0001$ & 128.08 & - & $<0.0001$ \\
\hline Conditioned soil (CS) & 5,12 & 11.98 & - & 0.0003 & 3.04 & - & 0.05 \\
\hline Spatial heterogeneity $(\mathrm{SH})$ & 2,276 & 5.21 & - & 0.006 & 15.16 & - & $<0.0001$ \\
\hline Coarse vs. Homogeneous & & - & -3.02 & 0.003 & - & -5.09 & $<0.0001$ \\
\hline Fine vs. Homogeneous & & - & -4.11 & $<0.0001$ & - & -4.06 & $<0.0001$ \\
\hline Coarse vs. Fine & & - & -0.90 & 0.37 & - & 1.00 & 0.32 \\
\hline PS $x$ CS & 25,276 & 5.10 & - & $<0.0001$ & 4.25 & - & $<0.0001$ \\
\hline PS $x$ SH & 10,276 & 3.19 & - & 0.0007 & 2.62 & - & 0.005 \\
\hline $\mathrm{CS} \times \mathrm{SH}$ & 10,276 & 3.33 & - & 0.0004 & 2.01 & - & 0.03 \\
\hline PS $x$ CS $x$ SH & 50,276 & 1.10 & - & 0.32 & 0.86 & - & 0.74 \\
\hline
\end{tabular}

(0-10\% of gridcells affected), but were quite high in J. vulgaris, particularly on its own soil (up to 75\%). We re-analysed the gridcell level LMMs on plant biomass using only those gridcells where no plants had died. This led to the same qualitative conclusions as the full analyses with the exception that for shoot biomass the main effect of space was now only marginally significant (Table S5.5). There were however clear interactions with spatial heterogeneity, indicating that it still affected the plant responses in the reduced dataset.

\section{Effect of spatial heterogeneity on the strength of direct plant-soil feedback}

Both above- and belowground plant biomass per container were strongly affected by the species conditioning the soil $\left(\mathrm{F}_{5,72}=17.16, P<0.0001\right.$ and $\mathrm{F}_{5,72}=5.77, P$ $=0.0002$ resp.; Table S5.6), but the response to each soil differed among the test species (aboveground biomass: $\mathrm{F}_{25,72}=2.05, P=0.0096$; belowground biomass: $\mathrm{F}_{25,72}=3.21, P=0.0001$; Fig. 5.5). The majority of plant species grew worse on their own soil than on other soils (Fig. 5.5; Table S5.6). Only aboveground biomass of A. capillaris was slightly higher (but this was not significant) on its own soil and this was not the case for belowground biomass. In addition, there were also clear indirect feedbacks. For instance, biomass of F. rubra was equally low in J. vulgarisconditioned soil as in its own soil (Fig. 5.5). Similarly, A. capillaris biomass was 


\section{Aboveground}

Plant: Ac

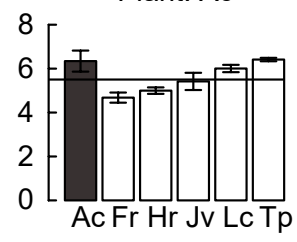

Plant: Fr

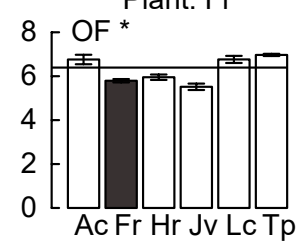

Plant: $\mathrm{Hr}$

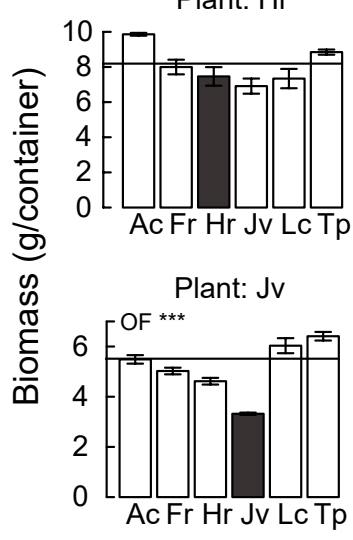

Plant: LC
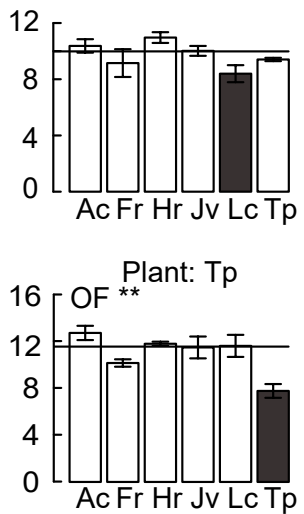

Belowground

Plant: Ac

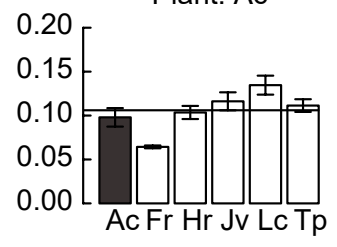

Plant: Fr

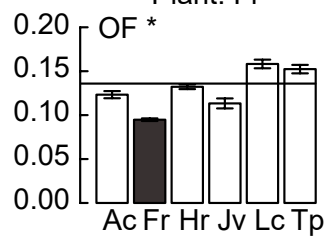

Plant: $\mathrm{Hr}$

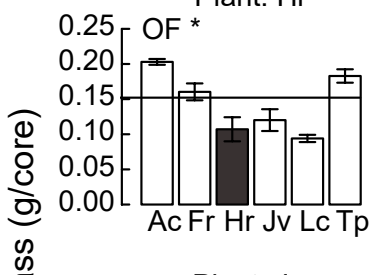

Plant: JV

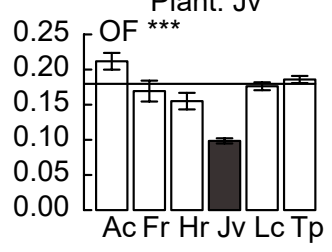

Plant: Lc

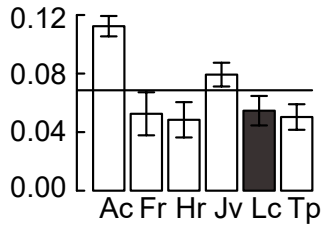

Plant: Tp

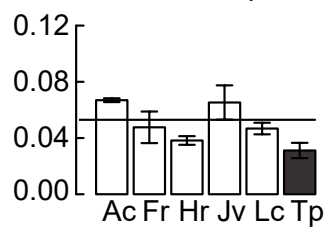

Soil conditioning species 
relatively high on its own soil, but this species produced $26 \%$ and $34 \%$ less biomass above- and belowground respectively in soil conditioned by $F$. rubra.

In contrast to the amount of biomass produced, the strength of the direct plantsoil feedback (own-foreign comparisons) did not change significantly among the spatial heterogeneity treatments (Table S5.7). As was the case in spatially homogeneous containers most direct PSF values were negative (Fig. S5.3), and only in the case of $J$. vulgaris shoot biomass did fine-grained heterogeneity lead to more strongly negative PSF values than observed under homogeneous conditions. Consequently, while plants produced on average less biomass in soils with spatially heterogeneous PSF, the generally negative effect of growing on self-conditioned soil was not aggravated by spatial PSF heterogeneity.

\section{Effect of soil conditioning on soil abiotic composition}

Soil conditioning caused differences in soil nitrate content and acidity (Table S5.1), but the relationship of the biomass of the test plant species at the end of phase 2 with the abiotic conditions was not consistent across species (Table S5.8). There was a generally positive influence of nitrogen and soil acidity on the biomass of non-legumes, while for the two legume species these relationships were reversed.

\section{Discussion}

Our results show that spatially heterogeneous plant-soil feedback effects result in lower plant biomass than predicted from spatially homogeneous treatments. In line with other studies, most plant species grew worse on soil conditioned by conspecifics ('own'-soil) than on heterospecific conditioned soil ('foreign'-soil; Petermann et al. 2008, Kulmatiski et al. 2008). However, when the biomass production on all four relevant homogeneous soil treatments (own and foreign soils) was averaged and compared to the biomass produced in heterogeneous PSF soils we found that the observed biomass was in general lower than expected. The effect of PSF heterogeneity on plant performance did however vary depending on the test plant species as well as on the species that conditioned the soil. The reduction in plant biomass due to spatial PSF heterogeneity did occur both on soil conditioned by conspecifics ('own') as well as heterospecifics ('foreign') and was insensitive to the grain of the heterogeneity (fine vs. coarse). If our study, with six different monocultures and six species-specific conditioned soils, can be extrapolated, the results suggest that the spatial PSF heterogeneity expected in the field (Ettema and Wardle 2002, Bezemer et al. 2010) may influence plant performance more strongly than was expected previously. However, as PSF can 
alter competitive ability (Casper and Castelli 2007) multispecies experiments that allow interspecific competition are needed to test this prediction.

In our experiment the spatially heterogeneous treatments consisted of four differently conditioned soils in the same container, while each of these soils in the homogeneous treatment were placed in different containers. Plant species cultivate different soil communities (Bever 1994, Grayston et al. 1998, Bezemer et al. 2010) by allowing different soil taxa to increase in abundance, which can affect plant performance when their populations exceed a certain threshold (Van der Putten et al. 1988, Hendriks et al. 2015b). Consequently, the spatially heterogeneous treatments likely had a higher diversity of taxa exceeding this threshold than the containers in the homogeneous treatment. In our experiment plant roots grew intermingled throughout whole containers (E.R.J. Wubs, pers. obs.). Hence, all plant individuals in the monocultures may have faced a more diverse pool of antagonists in spatially heterogeneous than in homogeneous soils. As a result this may increase the chances of a plant to encounter the specific pests and pathogens it is susceptible to. When triggered by the presence of their host the densities of antagonists may rapidly increase and hence this may explain why we observed that plant performance was reduced more in the heterogeneous treatments than what was predicted from the performance in spatially homogeneous soils. Importantly, the density of specific antagonists that are present in a conditioned soil will be lower in heterogeneous soils (e.g. lower volume of 'own' soil). Hence, this argument is only valid if the severity of the antagonists' effects is not strongly related to their initial density in the soil. Nevertheless, the higher level of diversity in the soil in the heterogeneous treatments also increases the scope for interactions among soil organisms, which may have increased the chances of co-infections. Co-infections of multiple pathogens can greatly aggravate diseases (De Rooij-Van der Goes 1995, Castillo et al. 2003, Tatineni et al. 2010) and have recently been suggested to play an important role in mediating Janzen-Connell effects (Benítez et al. 2013). As we grew plants in monocultures transmission rates of pathogens from plant to plant are likely to have been high (Mitchell et al. 2002, Schnitzer et al. 2011). The higher diversity of antagonists in heterogeneous soils combined with transmission of potentially co-infecting, antagonists from plant to plant may have resulted in the observed additional negative effect of PSF on plant performance in the heterogeneous treatments. Our results are in contrast to those of Hendriks et al. (2015b) who found that single plants had higher total biomass in pots with spatial PSF heterogeneity. We speculate that this may be due to the fact that the homogeneous control treatment in the study of Hendriks et al. (2015b) consisted 
of an artificially homogenized mixture of the four soils used in the heterogeneous treatment. Due to this mixing all soil biota were more or less homogeneously distributed around the plant, thus providing no enemy-free spaces in which the plant roots could avoid contact with their antagonists. In our study none of the conditioned soils were mixed. Instead, we tested plant performance on each of the conditioned soils separately, thus also allowing for enemy-free spaces in the spatially homogeneous treatment.

To test if the observed negative heterogeneity effects could be explained by the soil biota from the most growth-repressing soil (typically 'own' soil) colonizing the whole container, we also calculated the expected performance of plants on heterogeneous soils based not on the mean but on the performance in the homogeneous soil treatment with the strongest negative PSF. We expected that if the antagonists of the most growth-repressing soil take over the (heterogeneous) soil in the entire container they would drive the PSF effect at container level and consequently that this would predict plant performance in the heterogeneous containers. However, we found that on average plants performed better than predicted by the most growth-repressing soil (Fig. S5.4). Thus plant biomass of monocultures on spatially heterogeneous soils was less than the mean performance on those soils in homogeneous condition, but not as low as their biomass on the soil least conducive to its growth. Apparently, PSF in heterogeneous soils are not a simple function of the PSF measured in their constituent soils and include important non-additive interactions (Brandt et al. 2013, Hendriks et al. 2013). Our study suggests that monoculture growth will be poorer on soils uniformly conditioned by conspecifics ('own') then in heterogeneous soils where the direct PSF effect is somewhat diluted or delayed (Hendriks et al. 2013, 2015b). Importantly, however, plant establishment success will vary across different uniformly conditioned soils as the observed indirect feedbacks in our study underline. Moreover, in the field plant performance will also be importantly affected by competition with other species, which in turn are influenced by PSF (Casper and Castelli 2007).

The observed negative effects of spatial PSF heterogeneity could, alternatively, also have been caused by altered rooting patterns. Spatial heterogeneity in the soil can cause plants to adjust their rooting patterns, both in response to abiotic (Hutchings et al. 2003, De Kroon and Mommer 2006) and biotic changes (Hendriks et al. 2015b). Altered rooting patterns could have different costs of resource investment (Grime and Mackey 2002), which could have altered the biomass production over the longer term. It is important to point out that in our 
study root biomass was sampled using root cores and that not entire grids were measured. It is therefore possible that the changes in biomass observed in fact reflect only repositioning of the roots within the containers in response to patch quality (Hutchings et al. 2003, De Kroon and Mommer 2006, Hendriks et al. 2015b). However, given that many root cores were sampled per container and that we did not observe that a particular conditioned soil consistently attracted more roots than others we believe this may be unlikely. In addition, regression models predicting root biomass from shoot biomass show that including a separate slope for each of the levels of spatial heterogeneity does not significantly improve the model for any of the six test species (Table S5.3, Fig. S5.1). This suggests that the relationship between root and shoot biomass was consistent across the spatial PSF heterogeneity treatments. Even though this is indirect evidence, these data suggest that the observed heterogeneity effects belowground reflect differences in biomass production rather than differences in rooting patterns. More studies are needed to untangle the relative impact of different mechanisms and understand the consequences of spatially heterogeneous PSFs for plant community composition (Bever et al. 2010, Van der Putten et al. 2013). For instance, in contrast to the early- and mid-succession species used here, it would be interesting to test if latersuccessional plant species may benefit from spatial heterogeneity, because it can be expected that the diversity of mutualists (e.g. fungal endophytes) is also higher in heterogeneous soils and later successional species tend to have positive direct PSF (Kardol et al. 2006).

Contrary to our expectation we did not find consistent differences in performance due to fine-grained or coarse-grained heterogeneity (Mack and Bever 2014). This supports the idea that the negative impact of heterogeneity in our study was caused by the higher soil biodiversity in heterogeneous settings: in both coarse- and finegrained heterogeneity treatments the introduced soil biodiversity per container was the same. This does suggest that at the scale of our experiment $\left(0.0572 \mathrm{~m}^{2}\right.$ containers) the exact grain of the heterogeneity is unimportant, provided that the heterogeneity itself is present within a plant's zone of influence (Casper et al. 2003). As with heterogeneity in abiotic conditions (Hutchings et al. 2003), plant root systems can easily grow across these smaller patches and integrate over the differences in the biotic soil conditions, even though this does strongly affect the overall performance of the plant as antagonistic populations are likely to increase in time (Hendriks et al. 2015b). Future experiments should study whether plant roots respond differentially to abiotic and biotic soil heterogeneity, for instance in response to (volatile) signalling molecules released by the microbial community. 
This could provide insight into whether potential gains of soil exploration are actively weighed against the risks of encountering soil-borne antagonists. In addition, the spatial dynamics of PSFs - i.e. how far and how fast does PSF spread - is an open question. This will particularly depend on the mobility of the soil biota involved in the feedback (Bever et al. 2012). A better understanding of the spatial PSF dynamics would improve our understanding of spatial patterns in natural plant communities (Bever et al. 1997, Molofsky et al. 2002, Van der Putten 2003, Mack and Bever 2014), with potential application in intercropping designs.

In line with other studies on plant-soil feedback, we observed that in homogeneous soils for most of the tested plant species direct feedbacks ("own" vs "foreign" soil) were strongly negative (Bever 2003, Petermann et al. 2008, Kulmatiski et al. 2008). However, we also show that indirect feedbacks - the effect of a soil conditioned by one species on another species - can also have strong and variable effects. Both direct and indirect feedback can importantly change the performance of interacting plants and consequently affect community composition (Bever et al. 1997, Bever 2003), for instance during secondary succession (Van de Voorde et al. 2011). Interestingly, the strength of direct PSF itself was not affected by spatial heterogeneity in most cases. Only for shoot biomass of J. vulgaris did the strength of direct PSF increase as the grain of heterogeneity became finer. Consequently, while spatial PSF heterogeneity did reduce plant biomass in general, it did not affect plant biomass more strongly in soil patches conditioned by conspecifics ('own') than in the spatially homogeneous treatment. The stronger direct PSF of J. vulgaris in the fine-grained heterogeneous treatment is likely a consequence of increased plant mortality which was observed in that treatment. In general however plant mortality was not affected by spatial heterogeneity. We believe that plant mortality is an integral, albeit extreme, consequence of PSF. Since we replaced all seedlings that had died due to plant handling during transplantation we assume that the changes in mortality rates reported here are indeed a consequence of PSF. In any case, when plant biomass was analysed using only gridcells where no seedling had died the outcomes remained qualitatively unchanged.

Plant-soil feedbacks can be mediated both by changes in soil community composition and changes in abiotic conditions (Ehrenfeld et al. 2005). Soil conditioning in our experiment did lead to differences in abiotic conditions, particularly soil nitrogen content and acidity, and abiotic conditions were correlated to plant performance in the test phase to some extent. However, the relationships were not consistent across the test species. For instance, nitrogen content did 
promote plant growth for most species at least to some extent, especially so for F. rubra, but it reduced the performance of the legumes. In combination with the observation that most of the test species ( 5 out of 6 ) had a strong negative direct PSF, we interpret the lack of consistent relationships of plant performance with the measured abiotic conditions across species as evidence for the differential impact of altered soil communities during soil conditioning on plant performance (Kardol et al. 2006, Hendriks et al. 2013). It is important to note that although differences in abiotic conditions among the soils may have partly been responsible for the plant-soil feedbacks themselves, the effect of spatial PSF heterogeneity on plant performance described here is independent of these abiotic differences. This is because the comparison between plant performance in homogeneous and heterogeneous soils was always made while the plants were rooted in patches with the same soil, and these same soils were placed in containers with varying levels of spatial heterogeneity.

We conclude that spatial PSF heterogeneity affects plant performance in monocultures, and that plants on average grow less well relative to homogeneous soils. However, spatial heterogeneity did not aggravate the generally negative direct PSF found in most test species. Instead plant biomass was on average reduced in both 'own' as well as 'foreign' soil patches. In addition, the spatial grain of the PSF heterogeneity did not further affect plant performance. We hypothesize that the negative effect of spatial heterogeneity on plant performance results from the greater soil diversity encountered in spatially heterogeneous soils, making it more likely that plants will encounter their antagonists and increase the risk of coinfections with several pathogens. Our data suggest that spatial PSF heterogeneity in the field may negatively impact plant performance over and above the effects suggested by classical PSF studies. There are, however, important differences in direct and indirect PSFs in homogeneous treatments and thus soil patch quality as well. Finally, the spatial dynamics and dimensions of PSF effects need to be addressed in empirical and modelling studies to understand its consequences for plant communities in the field. 


\section{Supporting information}

Table S5.1. Soil chemistry and aboveground plant biomass of each focal species after eight weeks of conditioning the soil (phase 1).

Mean $( \pm S E)$ values and results of one-way ANOVA analyses of differences among conditioning species (different letters indicate significant differences among the species). $\mathrm{SOM}=$ soil organic matter, ns $=$ not significant, ${ }^{*} P<0.05,{ }^{* * *} P<0.001$.

\begin{tabular}{|c|c|c|c|c|c|c|}
\hline $\begin{array}{l}\text { Conditioning } \\
\text { species }\end{array}$ & $\begin{array}{l}\mathrm{PO}_{4}-\mathrm{P}^{*} \\
(\mathrm{mg} / \mathrm{kg})\end{array}$ & $\begin{array}{l}\mathrm{NO}_{3}-\mathrm{N} \\
(\mathrm{mg} / \mathrm{kg})\end{array}$ & $\begin{array}{l}\mathrm{NH}_{4}-\mathrm{N} \\
(\mathrm{mg} / \mathrm{kg})\end{array}$ & $\begin{array}{c}\text { Acidity }^{\dagger} \\
(\mathrm{pH})\end{array}$ & $\begin{array}{c}\text { SOM } \\
(\% \mathrm{w}: \mathrm{w})\end{array}$ & $\begin{array}{l}\text { Above- } \\
\text { ground } \\
\text { biomass } \\
\left(\text { g pot }^{-1}\right)\end{array}$ \\
\hline A. capillaris & $\begin{array}{l}95.50 \\
(1.47)\end{array}$ & $\begin{array}{l}3.94^{\mathrm{a}} \\
(0.28)\end{array}$ & $\begin{array}{c}6.24 \\
(0.61)\end{array}$ & $\begin{array}{l}5.98^{\mathrm{ab}} \\
(0.06)\end{array}$ & $\begin{array}{c}3.20 \\
(0.07)\end{array}$ & $\begin{array}{l}4.00^{c} \\
(0.08)\end{array}$ \\
\hline F. rubra & $\begin{array}{l}97.06 \\
(1.19)\end{array}$ & $\begin{array}{l}1.76^{\mathrm{b}} \\
(0.69)\end{array}$ & $\begin{array}{c}4.19 \\
(0.70)\end{array}$ & $\begin{array}{l}5.90^{\mathrm{ab}} \\
(0.03)\end{array}$ & $\begin{array}{l}3.20 \\
(0.09)\end{array}$ & $\begin{array}{l}3.52^{\mathrm{d}} \\
(0.07)\end{array}$ \\
\hline H. radicata & $\begin{array}{l}100.47 \\
(0.73)\end{array}$ & $\begin{array}{l}1.03^{\mathrm{b}} \\
(0.09)\end{array}$ & $\begin{array}{c}6.26 \\
(0.28)\end{array}$ & $\begin{array}{l}5.89^{\mathrm{ab}} \\
(0.02)\end{array}$ & $\begin{array}{l}3.15 \\
(0.03)\end{array}$ & $\begin{array}{l}3.97^{c} \\
(0.09)\end{array}$ \\
\hline J. vulgaris & $\begin{array}{c}100.30 \\
(5.31)\end{array}$ & $\begin{array}{l}0.81^{\mathrm{b}} \\
(0.14)\end{array}$ & $\begin{array}{c}5.67 \\
(0.78)\end{array}$ & $\begin{array}{l}5.99^{\mathrm{b}} \\
(0.03)\end{array}$ & $\begin{array}{c}3.32 \\
(0.01)\end{array}$ & $\begin{array}{l}2.52^{\mathrm{e}} \\
(0.05)\end{array}$ \\
\hline L. corniculatus & $\begin{array}{c}103.70 \\
(0.78)\end{array}$ & $\begin{array}{l}3.40^{\mathrm{a}} \\
(0.54)\end{array}$ & $\begin{array}{c}6.21 \\
(0.71)\end{array}$ & $\begin{array}{l}5.89^{\mathrm{ab}} \\
(0.03)\end{array}$ & $\begin{array}{l}3.05 \\
(0.16)\end{array}$ & $\begin{array}{l}8.39^{\mathrm{b}} \\
(0.22)\end{array}$ \\
\hline T. pratense & $\begin{array}{l}98.70 \\
(4.52)\end{array}$ & $\begin{array}{l}0.92^{\mathrm{b}} \\
(0.17)\end{array}$ & $\begin{array}{l}7.05 \\
(0.22)\end{array}$ & $\begin{array}{l}5.82^{\mathrm{a}} \\
(0.01)\end{array}$ & $\begin{array}{c}3.15 \\
(0.24)\end{array}$ & $\begin{array}{l}10.87^{\mathrm{a}} \\
(0.33)\end{array}$ \\
\hline ANOVA F-test & $0.93^{\mathrm{ns}}$ & $12.38^{* * *}$ & $2.63^{\text {ns }}$ & $3.23^{*}$ & $0.76^{\mathrm{ns}}$ & $272.53^{* * *}$ \\
\hline
\end{tabular}

${ }^{*}$ P-Olsen extraction, ${ }^{\dagger} \mathrm{H}_{2} \mathrm{O}$ extraction 
Table S5.2. Analysis of variance (SS type I) table of mixed models of plant biomass (shoots and root separately) in the spatial PSF experiment at gridcell level, with a 16-level random effect for gridcell (i.e. instead of 3 based on rotational symmetry; c.f. Table 5.2).

The models include nested random effects for soil replicate, container, and gridcell. Presented are degrees of freedom (d.f.), F-values, and p-values.

\begin{tabular}{|c|c|c|c|c|c|}
\hline \multirow[b]{2}{*}{ Term } & \multirow[b]{2}{*}{ d.f. } & \multicolumn{2}{|c|}{ Shoot biomass } & \multicolumn{2}{|c|}{ Root biomass } \\
\hline & & $\mathrm{F}$ & p-value & $\mathrm{F}$ & p-value \\
\hline Plant species (PS) & 5,276 & 134.71 & $<0.0001$ & 128.20 & $<0.0001$ \\
\hline Conditioned soil (CS) & 5,12 & 12.04 & 0.0002 & 3.04 & 0.05 \\
\hline Spatial heterogeneity $(\mathrm{SH})$ & 2,276 & 5.22 & 0.0006 & 15.16 & $<0.0001$ \\
\hline PS $\times$ CS & 25,276 & 5.10 & $<0.0001$ & 4.25 & $<0.0001$ \\
\hline PS $x$ SH & 10,276 & 3.19 & 0.0007 & 2.62 & 0.005 \\
\hline $\mathrm{CS} \times \mathrm{SH}$ & 10,276 & 3.33 & 0.0004 & 2.01 & 0.03 \\
\hline $\mathrm{PS} \times \mathrm{CS} \times \mathrm{SH}$ & 50,276 & 1.10 & 0.32 & 0.86 & 0.74 \\
\hline
\end{tabular}


Table S5.3. Relationship between root and shoot biomass for the six test species (c.f. Fig. S5.1).

A) Results are shown for F-tests of coincidental regression lines (following Zar 1999, p. 375) among above- and belowground biomass for the three levels of spatial heterogeneity at container level. Belowground biomass was used as the response variable, shoot biomass as the predictor. B) F-test comparing whether shoot biomass was a predictor of root biomass in the test species across all three levels of spatial heterogeneity. C) Regression slopes (Beta) and confidence intervals (95\% CI Beta) for the relationship between shoot and root biomass for each test species separated by spatial heterogeneity treatment level (uniform, coarse-, or fine-grained).

\begin{tabular}{lcccccccc}
\hline & $\begin{array}{c}\text { A) Coincident } \\
\text { regressions }\end{array}$ & \multicolumn{2}{c}{$\begin{array}{c}\text { B) Simple linear } \\
\text { regression }\end{array}$} & \multicolumn{3}{c}{ C) Regression per treatment level } \\
\hline Species & $\mathrm{F}_{4.24}$ & $\mathrm{p}$-value & $\mathrm{F}_{1.28}$ & $\mathrm{p}$-value & Treatment & $\mathrm{N}$ & Beta & 95\% CI Beta \\
\hline A. capillaris & 2.34 & 0.084 & 0.61 & 0.44 & Uniform & 18 & 0.07 & {$[-0.191: 0.333]$} \\
& & & & & Coarse & 6 & -0.26 & {$[-0.901: 0.386]$} \\
& & & & & Fine & 6 & 0.15 & {$[-0.177: 0.479]$} \\
\hline F. rubra & 0.62 & 0.66 & 2.35 & 0.14 & Uniform & 18 & 0.33 & {$[0.069: 0.589]$} \\
& & & & & Coarse & 6 & -0.40 & {$[-2.661: 1.857]$} \\
& & & & & Fine & 6 & -0.02 & {$[-1.100: 1.051]$} \\
\hline H. vadicata & 0.56 & 0.70 & 6.74 & 0.02 & Uniform & 18 & 0.27 & {$[0.012: 0.532]$} \\
& & & & & Coarse & 6 & 0.14 & {$[-0.058: 0.333]$} \\
& & & & & Fine & 6 & -0.13 & {$[-0.856: 0.591]$} \\
\hline J. vulgaris & 1.80 & 0.16 & 24.60 & $<0.0005$ & Uniform & 18 & 0.48 & {$[0.282: 0.685]$} \\
& & & & & Coarse & 6 & -0.08 & {$[-2.172: 2.021]$} \\
& & & & & Fine & 6 & 0.02 & {$[-1.274: 1.322]$} \\
\hline L. corniculatus & 0.45 & 0.77 & 9.67 & 0.004 & Uniform & 18 & 0.14 & {$[-0.043: 0.324]$} \\
& & & & & Coarse & 6 & 0.31 & {$[-0.493: 1.120]$} \\
& & & & & Fine & 6 & 0.30 & {$[-0.048: 0.655]$} \\
\hline T. pratense & 0.58 & 0.69 & 14.72 & $<0.0005$ & Uniform & 18 & 0.09 & {$[0.026: 0.161]$} \\
& & & & & Coarse & 6 & 0.10 & {$[-0.054: 0.258]$} \\
& & & & & Fine & 6 & 0.06 & {$[-0.132: 0.247]$} \\
\hline
\end{tabular}




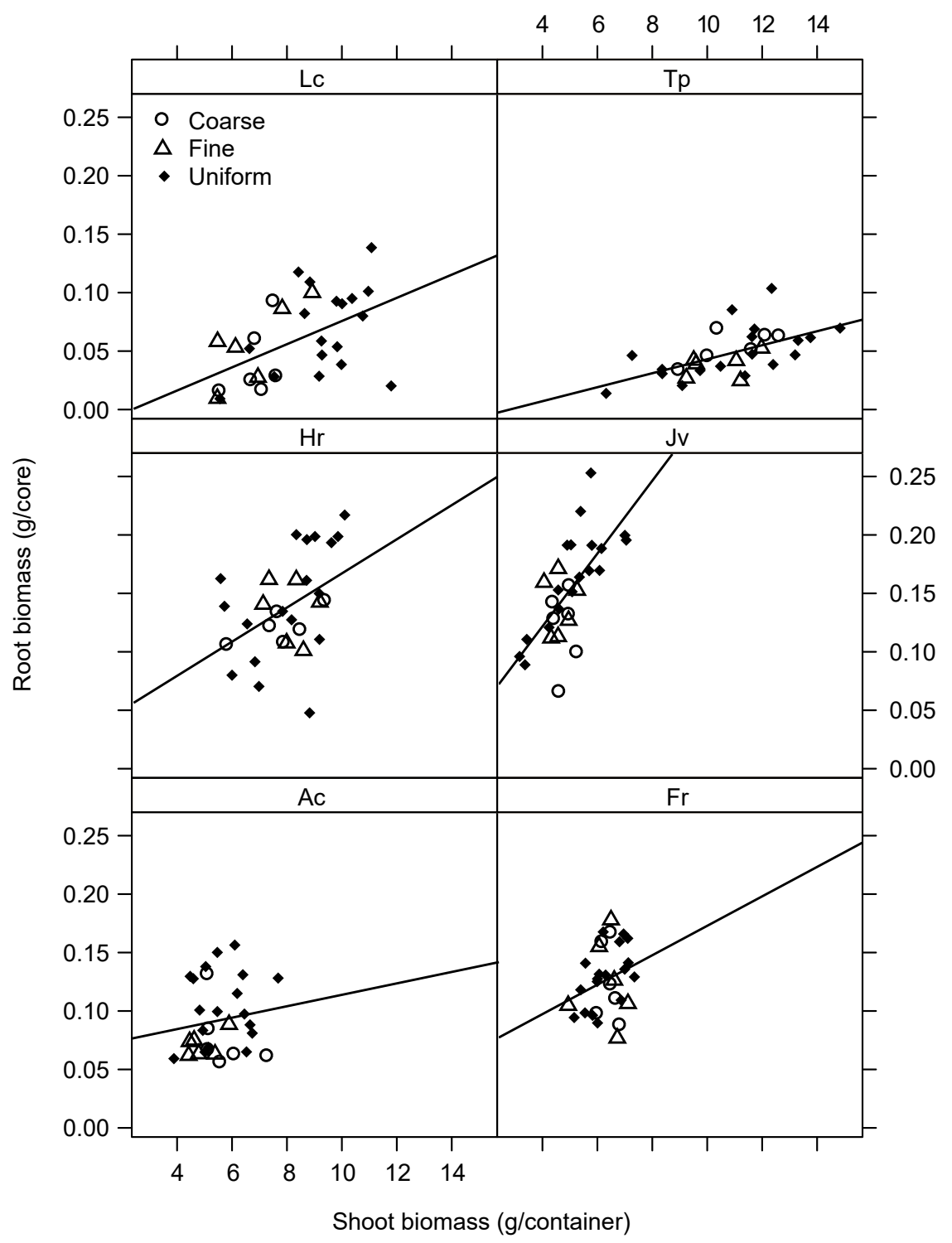

Fig. S5.1. Relationship between root and shoot biomass for each of the six test species.

The different symbols represent observations from the uniform (diamonds), coarse- (circles), and fine-grained (triangles) spatial PSF treatment. Regression lines are shown for each species using all data points. Ac: Agrostis capillaris, Fr: Festuca rubra, Hr: Hypochaeris radicata, Jv: Jacobaea vulgaris, Lc: Lotus corniculatus, Tp: Trifolium pratense. 
Table S5.4. Analysis results of a generalized linear mixed model testing the effects of plant species, soil conditioning and spatial heterogeneity on plant mortality at gridcell level.

Each model term was tested using a likelihood-ratio (LR) test. The model includes nested random effects for soil replicate, container, and gridcell. Plant mortality was modelled as a binary variable taking a value of one if either or both of the plants per gridcell had died. Presented are degrees of freedom (d.f.), likelihood-ratio (LR) statistics, and p-values.

\begin{tabular}{lccc}
\hline Term & d.f. & LR-statistic & p-value \\
\hline Plant species (PS) & 5 & 93.00 & 0.0001 \\
Conditioned soil (CS) & 5 & 16.47 & 0.0006 \\
Spatial heterogeneity (SH) & 2 & 2.72 & 0.26 \\
PS x CS & 25 & 78.20 & $<0.0001$ \\
PS x SH & 10 & 19.29 & 0.04 \\
CS x SH & 10 & 14.03 & 0.17 \\
PS x CS x SH & 50 & 66.50 & 0.06 \\
\hline
\end{tabular}



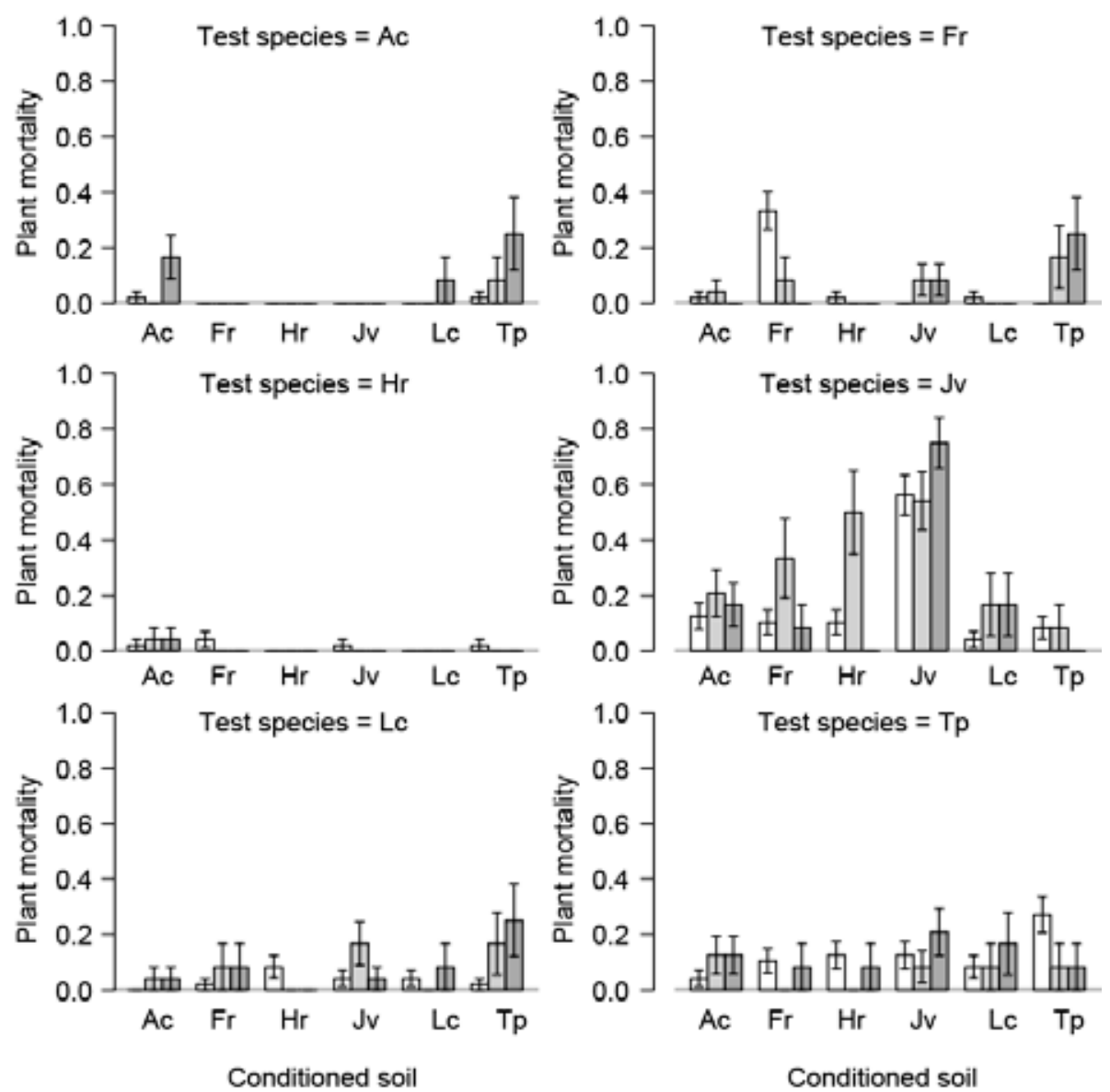

Fig. S5.2. Plant mortality (mean $\pm S E$ ) in the spatial PSF experiment.

Mortality was recorded as a binary variable which takes the value one when either one or both of the plants per gridcell had died. For statistical analysis see Table S5.3. 
Table S5.5. Analysis of variance (SS type I) table of mixed models of plant biomass (shoots and root separately) in the spatial PSF experiment at gridcell level, restricted to observations without plant mortality (c.f. Table 5.2).

The models include nested random effects for soil replicate, container, and gridcell. Presented are degrees of freedom (d.f.), F-values, and p-values, as well as the results of the overall planned contrasts (z-values) among spatial heterogeneity treatments.

\begin{tabular}{|c|c|c|c|c|c|c|c|}
\hline \multirow[b]{2}{*}{ Term/contrast } & \multirow[b]{2}{*}{ d.f. } & \multicolumn{3}{|c|}{ Shoot biomass } & \multicolumn{3}{|c|}{ Root biomass } \\
\hline & & $\mathrm{F}$ & Z & p-value & $\mathrm{F}$ & $\mathrm{Z}$ & p-value \\
\hline Plant species (PS) & 5,273 & 113.11 & - & $<0.0001$ & 125.88 & - & $<0.0001$ \\
\hline Conditioned soil (CS) & 5,12 & 9.35 & - & 0.0008 & 3.16 & - & 0.05 \\
\hline Spatial heterogeneity (SH) & 2,273 & 2.65 & - & 0.07 & 12.74 & - & $<0.0001$ \\
\hline Coarse vs. Homogeneous & & - & -2.66 & 0.008 & - & -4.89 & $<0.0001$ \\
\hline Fine vs. Homogeneous & & - & -3.54 & 0.0004 & - & -3.52 & 0.0004 \\
\hline Coarse vs. Fine & & - & -0.77 & 0.44 & - & 1.31 & 0.19 \\
\hline PS $x$ CS & 25,273 & 3.14 & - & $<0.0001$ & 3.76 & - & $<0.0001$ \\
\hline $\mathrm{PS} \times \mathrm{SH}$ & 10,273 & 2.56 & - & 0.006 & 2.72 & - & 0.003 \\
\hline $\mathrm{CS} \times \mathrm{SH}$ & 10,273 & 1.73 & - & 0.07 & 1.71 & - & 0.08 \\
\hline PS $x$ CS $x$ SH & 50,273 & 1.43 & - & 0.04 & 0.79 & - & 0.85 \\
\hline
\end{tabular}


Table S5.6. Analysis of variance (SS type I) table of plant biomass in homogeneous containers (i.e. six test species $x 6$ conditioned soil types).

Presented are degrees of freedom (d.f.), F-values and p-values, as well as the results of planned contrasts (Z-values) for the effect of growing on 'own' vs. 'foreign' soil.

\begin{tabular}{lccccccc}
\hline & & \multicolumn{2}{c}{ Shoot biomass } & & \multicolumn{2}{c}{ Root biomass } \\
\cline { 3 - 4 } \cline { 6 - 7 } Terms & d.f. & F & p-value & & F & p-value \\
\hline Plant species & 5 & 58.90 & $<0.0001$ & & 48.84 & $<0.0001$ \\
Conditioned soil & 5 & 17.16 & $<0.0001$ & & 5.77 & 0.0002 \\
Plant x Conditioned soil & 25 & 2.05 & 0.01 & & 3.21 & 0.0001 \\
Residual & 72 & & & & & \\
\hline
\end{tabular}

Own-Foreign contrasts

\begin{tabular}{lcccc} 
Species & $\mathrm{Z}$ & $\mathrm{p}$-value & $\mathrm{Z}$ & $\mathrm{p}$-value \\
\hline A. capillaris & 1.54 & 0.16 & -0.47 & 0.64 \\
F. rubra & -2.26 & 0.05 & -2.38 & 0.03 \\
H. radicata & -0.95 & 0.34 & -2.61 & 0.03 \\
J. vulgaris & -6.57 & $<0.0001$ & -4.70 & $<0.0001$ \\
L. corniculatus & -1.50 & 0.16 & -0.84 & 0.48 \\
T. pratense & -3.03 & 0.007 & -1.27 & 0.31 \\
\hline
\end{tabular}



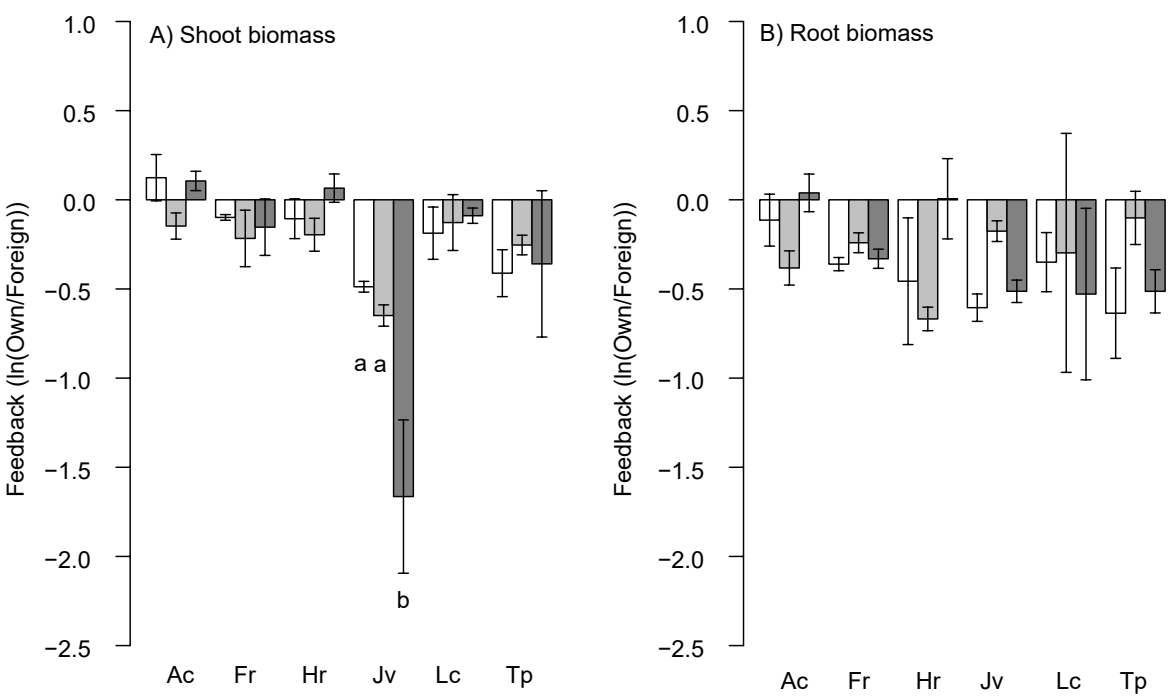

Test plant species

Fig. S5.3. Strength of feedback (log-ratio of biomass on 'own' and 'foreign' soil; mean $\pm \mathrm{SE}$ ) of the six test plant species in three spatial heterogeneity treatments (uniform: white bars, coarse: light grey bars, and fine: dark grey bars).

Ac: Agrostis capillaris, Fr: Festuca rubra, Hr: Hypochaeris radicata, Jv: Jacobaea vulgaris, Lc: Lotus corniculatus, Tp: Trifolium pratense.

Table S5.7. Analysis of variance (SS type I) table of 'own'-'foreign' soil feedback values for six test plant species in response to different levels of spatial PSF heterogeneity.

Presented are degrees of freedom (d.f.), F-values and p-values.

\begin{tabular}{lcccccc}
\hline & & \multicolumn{2}{c}{ Shoot biomass } & & \multicolumn{2}{c}{ Root biomass } \\
\cline { 3 - 4 } \cline { 6 - 7 } Term & d.f. & F & p-value & & F & p-value \\
\hline Plant species (PS) & 5 & 12.29 & $<0.0001$ & & 0.55 & 0.74 \\
Spatial heterogeneity (SH) & 2 & 1.22 & 0.31 & & 0.42 & 0.66 \\
PS x SH & 10 & 2.89 & 0.009 & & 0.98 & 0.48 \\
Residual & 36 & & & & & \\
\hline
\end{tabular}


Table S5.8. Spearman correlation coefficients for the relationship of growth of the test species (above and belowground) and measured abiotic conditions of the soil after soil conditioning.

Significant correlations $(\mathrm{n}=18, \mathrm{p}<0.05)$ are given in bold face. Only containers with homogeneous soils were used for these analyses. $\mathrm{AGB}=$ aboveground biomass, $\mathrm{BGB}=$ belowground biomass, $\mathrm{P} 1 \mathrm{AGB}=$ phase 1 aboveground biomass, $\mathrm{SOM}=$ soil organic matter.

\begin{tabular}{lccccccc}
\hline Test species & Response & $\mathrm{PO}_{4}$ & $\mathrm{NO}_{3}$ & $\mathrm{NH}_{4}$ & Acidity & SOM & P1 AGB \\
\hline A. capillaris & $\mathrm{AGB}$ & -0.01 & 0.22 & -0.13 & -0.04 & -0.18 & 0.44 \\
& $\mathrm{BGB}$ & 0.07 & 0.14 & 0.45 & -0.07 & -0.08 & 0.32 \\
F. rubra & $\mathrm{AGB}$ & -0.09 & $\mathbf{0 . 5 6}$ & 0.41 & -0.17 & -0.46 & $\mathbf{0 . 7 6}$ \\
& $\mathrm{BGB}$ & 0.43 & 0.18 & $\mathbf{0 . 5 9}$ & $\mathbf{- 0 . 4 9}$ & -0.30 & $\mathbf{0 . 7 7}$ \\
H. radicata & $\mathrm{AGB}$ & -0.38 & 0.38 & -0.04 & -0.13 & -0.11 & 0.36 \\
& $\mathrm{BGB}$ & $\mathbf{- 0 . 5 0}$ & 0.21 & 0.37 & -0.09 & 0.01 & 0.23 \\
J. vulgaris & $\mathrm{AGB}$ & 0.02 & 0.41 & 0.27 & $\mathbf{- 0 . 5 9}$ & -0.27 & $\mathbf{0 . 8 1}$ \\
& $\mathrm{BGB}$ & -0.15 & $\mathbf{0 . 4 8}$ & 0.17 & -0.31 & -0.21 & $\mathbf{0 . 5 4}$ \\
L. corniculatus & $\mathrm{AGB}$ & -0.07 & -0.29 & -0.07 & 0.02 & $\mathbf{0 . 4 8}$ & -0.20 \\
& $\mathrm{BGB}$ & $\mathbf{- 0 . 5 7}$ & -0.03 & -0.34 & 0.28 & 0.29 & -0.18 \\
T. pratense & AGB & 0.03 & 0.17 & -0.42 & 0.32 & 0.13 & -0.24 \\
& $\mathrm{BGB}$ & -0.30 & 0.07 & -0.47 & 0.27 & 0.22 & -0.27 \\
\hline
\end{tabular}




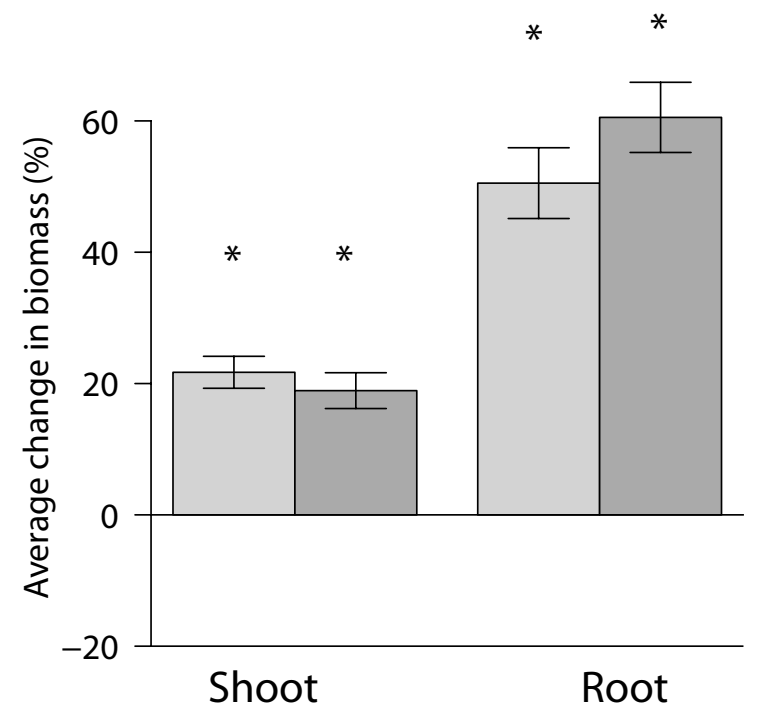

Fig. S5.4. The effect (mean \pm SE) of spatial PSF heterogeneity on plant performance averaged over the test species relative to their performance on the homogeneous soil where they produced the lowest (average) biomass (c.f. Fig. 5.2A).

The effects of both coarse (light grey) and fine (dark grey) scale heterogeneity are shown. Starts indicate significant differences from zero. 
CHAPTER 6

Spatial plant-soil feedback heterogeneity promotes plant diversity through equalizing competitive ability

E. R. Jasper Wubs and T. Martijn Bezemer 


\section{Abstract}

Plant-soil feedbacks have been identified as a key driver of local (alpha) diversity of plants in competitive communities. However, while it has been shown that spatial heterogeneity can alter plant performance and competitive interactions, there is no proof of principle that spatial PSF heterogeneity may enhance species diversity. Using a grassland model system we show that spatially heterogeneous PSFs maintained a higher plant diversity then when feedbacks were spatially homogeneous. On soils conditioned by one species, heterospecifics consistently outperformed the focal species leading to altered competitive hierarchies across mono-conditioned soils. On heterogeneous soils with patches of soil conditioned by different plant species, however, performance of all competing species was intermediate to the best and the worst mono-conditioned soils, leading to higher community evenness through equalizing competitive abilities of the plants. Interestingly, while spatially heterogeneous soils lead to higher diversity than the mono-conditioned soils, uniform soils that were conditioned simultaneously by all competing species, i.e. soil conditioned by mixed plant communities, also had high levels of plant diversity. This suggests that higher diversity was more strongly related to the number of plant species that conditioned the soil, than the spatial grain of the PSF heterogeneity. In conclusion, our data demonstrate directly that PSFs play a role in maintaining plant alpha diversity. Future studies need to investigate the importance of PSFs in the field relative to e.g. resource niche differentiation and the colonization-competition trade-off.

\section{Introduction}

A long standing question in ecology is how high plant species diversity is maintained at local spatial scales (Hutchinson 1961, Wilson et al. 2012), as competitors are known to exclude one another (Hardin 1960). Several authors have proposed that at small spatial scales plant antagonists, particularly soil-borne pathogens, may act as key drivers of plant diversity (Bradley et al. 2008, De Kroon et al. 2012, Bever et al. 2015, Bennett and Cahill 2016).

When growing in soil, plants induce changes in the composition of the soil community (Bezemer et al. 2010, Lundberg et al. 2012) and these changes, in turn, affect plant performance, a phenomenon known as plant-soil feedback (Bever 1994, 2003, Van der Putten et al. 2013). Most direct PSF effects, i.e. the effect of growing on self-conditioned soil, are reported to be negative and this may prevent species from becoming mono-dominant in the community (Petermann et al. 2008, Kulmatiski et al. 2008). The particular soil organisms that cause the 
PSF effect, such as soil pathogen species, may vary between soils and may be different for each plant species in the community (Bever et al. 2015). Furthermore, interactions with other soil organisms can strongly alter the effects of mutualists and pathogens on plants (Morris et al. 2007, Bradley et al. 2008, Hersh et al. 2012). Hence, PSF studies quantify the net effect of soil community changes on plant performance (Bever et al. 1997, Van der Putten et al. 2013).

While negative plant-soil feedbacks may prevent monocultures, it is unclear whether this will also lead to plant diversity. Theoretical models of spatial plant communities show that PSF effects can mediate coexistence if they are highly localized in space (Bonanomi et al. 2005, Fukami and Nakajima 2013, Mack and Bever 2014, Abbott et al. 2015). Recent empirical work has confirmed that spatial heterogeneity in PSFs can affect plant performance (Brandt et al. 2013, Hendriks et al. 2015a, Chapter 5), establishment of new species (Burns and Brandt 2014), and can alter competitive hierarchies (Hendriks et al. 2015b). However, empirical evidence that heterogeneity of plant-soil feedback enhances plant diversity in communities is currently lacking (Hendriks et al. 2015b). In addition, it is has not been tested how the spatial grain of PSF heterogeneity affects plant performance in mixed plant communities.

Here we test in model grassland communities whether spatial PSF heterogeneity, at two grain sizes (Coarse and Fine; Fig. 6.1), drives plant alpha diversity at a scale where plants interact with neighbouring plants and with the soil community. We hypothesize that plant diversity is higher in the spatially heterogeneous treatments. This is because in uniform soil conditioned by one species (mono-conditioned) heterospecific plants can become dominant as they do not encounter their own soil-borne antagonists within their rooting zones (Casper et al. 2003), while in heterogeneous soils, conditioned by multiple species, each species is kept in check by its own set of soil-borne antagonists. Finally, the exact grain of spatial PSF heterogeneity will not affect plant diversity as long as the same suite of soil biota are present within the soil volume that the plant community has access to. In that case, each species is equally likely to encounter its enemies regardless of their exact spatial location (Chapter 5). Consequently, we predict that homogeneous soils conditioned by multiple species (mixed-conditioned) will have the same effect on plant diversity as spatially heterogeneous treatments. We show that spatial PSF heterogeneity enhances plant diversity relative to mono-conditioned uniform soils, demonstrating that PSFs can be drivers of plant diversity at small spatial scales. 


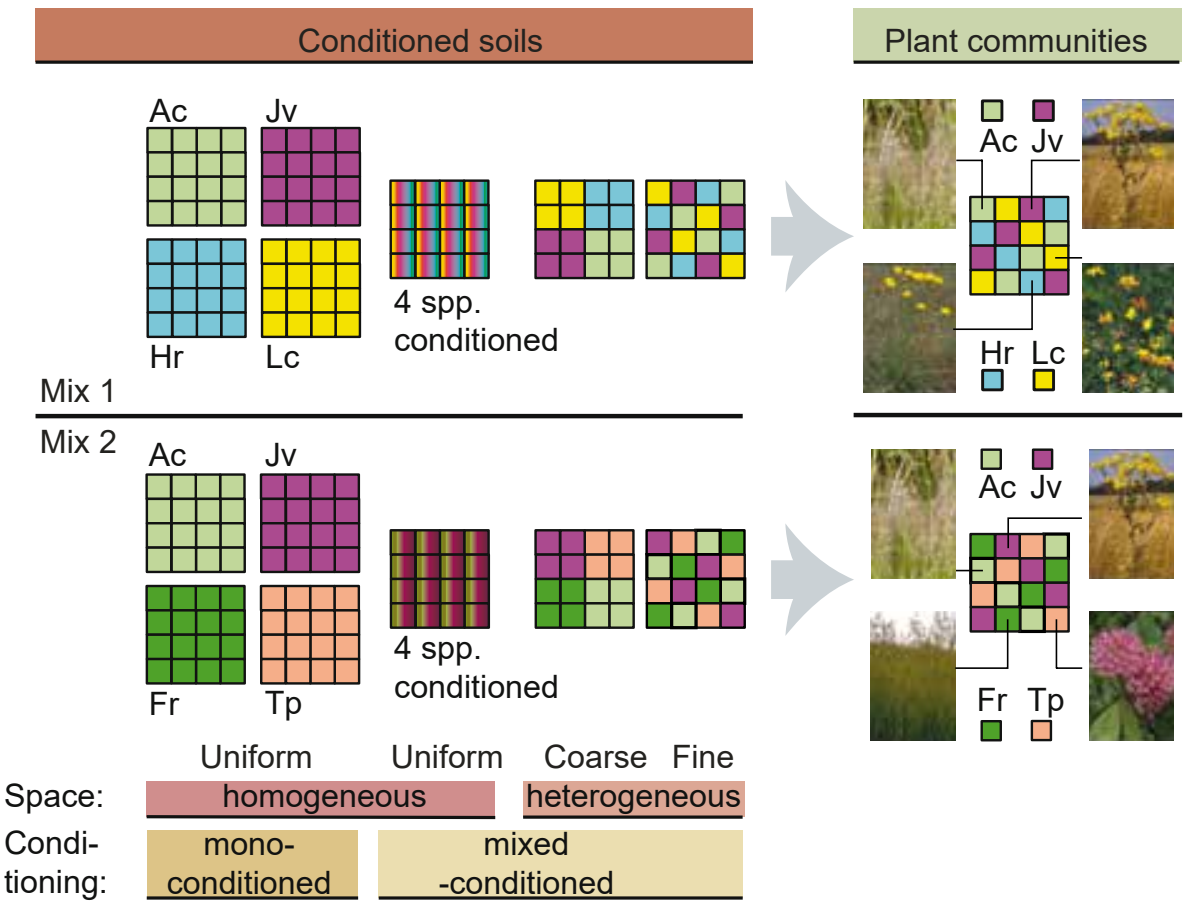

\section{Fig. 6.1. Experimental design.}

Two plant communities (mix 1 and 2) were planted each on seven conditioned soils with different levels of spatial heterogeneity. Soils were either homogeneous or heterogeneous (fine- or coarsegrained heterogeneity). Heterogeneous soils were conditioned by the same four species as in the respective plant mixture. The homogeneous soils (uniform) were either conditioned by one species (mono-conditioned) or simultaneously conditioned by four species in mixture (mixedconditioned). Ac= Agrostis capillaris, $\mathrm{Fr}=$ Festuca rubra, $\mathrm{Hr}=$ Hypochaeris radicata, $\mathrm{Jv}=$ Jacobaea vulgaris, $\mathrm{Lc}=$ Lotus corniculatus, $\mathrm{T} \mathrm{p}=$ Trifolium pratense.

\section{Materials and Methods}

To test our hypotheses, we conducted a glasshouse plant-soil feedback experiment where we grew two plant communities on four soil-conditioning treatments, where we explicitly manipulated the spatial PSF heterogeneity (Fig. 6.1). Six plant species were selected that are typical for old-fields on sandy soils in northwest Europe, with two representatives each for grass, forb and legume functional groups. They were: Agrostis capillaris L. and Festuca rubra L., Hypochaeris radicata L. and Jacobaea vulgaris Gaertn. (syn. Senecio jacobaea L.), and Lotus corniculatus L. and Trifolium pratense L. Plant-soil feedback experiments typically consist of 
two phases, first one where plants condition the soil (conditioning phase) and subsequently a phase where the effects of the soil conditioning on plant growth are tested (test or feedback phase).

\section{Phase 1: soil conditioning}

We collected topsoil (to $30 \mathrm{~cm}$ deep) from an ex-arable grassland (Mossel, Planken Wambuis, Ede, the Netherlands, GPS: $52^{\circ} 040 \mathrm{~N} 05^{\circ} 450 \mathrm{E}$ ) that had been under restoration for 17 years. Bags were a priori allocated to one of three replicates and these soil replicates were kept separate throughout the experiment. The soil was sieved ( $5 \mathrm{~mm}$ mesh size) and used to fill containers $(17 \times 17 \times 17 \mathrm{~cm} ; 5 \mathrm{~kg}$ of soil per container). We subsequently conditioned the soil for eight weeks, by growing all six plant species in monocultures in the soil in a glasshouse. All seeds were surface-sterilized ( $1 \mathrm{~min}$. in $<2.5 \% \mathrm{NaClO}$ solution), rinsed with water and allowed to germinate on sterilized glass beads in a climate chamber (16:8h daynight cycle, continuous $20{ }^{\circ} \mathrm{C}$ ) for two weeks. Sixteen individuals of a species were planted in each container and there were 58 containers per species, except for $A$. capillaris and J. vulgaris with 77 containers each. More soil of the latter two species was needed to create the spatially heterogeneous treatments in the test phase (see below). In addition, we implemented a mixed-conditioning treatment where two sets of four species simultaneously grew in the soil. The first set was planted with F. rubra, H. radicata, J. vulgaris and L. corniculatus, while the second set was planted with A. capillaris, F. rubra, J. vulgaris, and T. pratense. Here we planted four individuals per species in a latin square design. In both sets 37 pots of soil were conditioned. Containers were placed randomly in the greenhouse and were allowed to grow in the greenhouse (16:8h day:night, natural light supplemented with $600 \mathrm{~W}$ metal-halide lamps, 1 per $4 \mathrm{~m}^{-2}$, approx. $225 \mu \mathrm{mol}$ light quanta $\mathrm{m}^{-2} \mathrm{~s}^{-1}$ at plant level, $21: 16{ }^{\circ} \mathrm{C}$ day:night, $50-70 \%$ relative humidity) for 8 weeks. Subsequently, shoot biomass was clipped and large root systems were removed from the soil of each pot. Soil from containers in which the same species had grown and that were a priori allocated to the same soil-replicate were pooled and homogenized. To obtain a sufficient amount of soil for the experiment, each of the soil replicates was mixed with sterilized ( $>25 \mathrm{KGray}$ gamma radiation, Isotron, Ede, the Netherlands) field soil collected from the same site in a 8.4:1.6 (conditioned: sterile w:w) ratio. From each of the homogenized soil replicates a sample $(200 \mathrm{~g})$ was taken for chemical analysis upon addition of the sterilized soil. We measured mineral nitrogen (KCl-extraction), $\mathrm{PO}_{4}$ (P-Olsen extraction) and soil organic matter (ashed at $430^{\circ} \mathrm{C}$ for $24 \mathrm{~h}$ ) content as well as soil acidity (in 1:2.5 $\mathrm{w}: \mathrm{w}$ dry soil : water suspensions; see Table S6.1). Furthermore, three soil samples, 
one per soil replicate, were taken from each of the mono-conditioned soils $(\mathrm{n}=18)$ and analysed for differences in fungal community composition using Terminal Restriction Fragment Length Polymorphism (T-RFLP) analysis of the ITS marker (see Supplementary Information Methods S6.1 for the protocol).

\section{Phase 2: test (or feedback) phase}

In the test phase three different levels of spatial PSF heterogeneity were created (spatially homogeneous, spatially heterogeneous coarse-grained, and spatially heterogeneous fine-grained) each applied to different large containers (Fig. 6.1). Each container (length $\mathrm{x}$ width $\mathrm{x}$ height: $26 \times 22 \times 22 \mathrm{~cm}$ ) was divided with a custom-made metal grid into $4 \times 4$ cells, each with a surface area of $-35 \mathrm{~cm}^{2}$ (the length and width of the cells differed slightly to account for the rounded corners of the containers), extending to the bottom of the container. The size of the gridcells was chosen because at this grain size systematic differences in soil community composition were detected among plant species in open communities in the field (Bezemer et al. 2010). In each container, independent of the treatment, all 16 gridcells were filled individually and any given gridcell was always filled with a single conditioned soil. Immediately after filling the containers the grids were removed so that during the test phase the soil patches in each container were in full contact. For the uniform treatment all cells in a container were filled with one conditioned soil (either mono- or mixed-conditioned), while for spatially heterogeneous treatments (coarse- and fine-grained) gridcells were filled with soil mono-conditioned by four different species (Fig. 6.1). The four soils in the fine-grained treatment were applied following a Latin square design, while for the coarse-grained treatment four contiguous square blocks of four cells each were created in each container. The two spatially heterogeneous treatments (coarse- and fine-grained) were created with two different mixes of soil conditioned by four plant species (soil mix). Soil mix 1 consisted of soils conditioned by A. capillaris, $H$. radicata, J. vulgaris, and L. corniculatus; Soil mix 2 consisted of $A$. capillaris, F. rubra, J. vulgaris, and T. pratense. Consequently, both soil mixes had at least one representative each of the grass, forb and legume plant functional types. Containers in the mixed-conditioned uniform treatment received the soil that was simultaneously conditioned by four species in all gridcells. Soils from all six focal species were used separately to create spatially homogeneous containers with mono-conditioned soil. Subsequently, the conditioned soils were planted with two mixtures of four plant species, which were the same as the species that conditioned the two soil mixes mentioned above. Data from both plant mixes were analysed only when growing on soils in their own mix. Consequently, each plant mixture grew 
on seven soil-by-spatial heterogeneity treatments (4 mono-conditioned uniform, 1 mixed-conditioned uniform, 1 coarse heterogeneous and 1 fine heterogeneous). The four species were planted in a Latin square design which was selected randomly with the constraint that each plant species would be planted on all four soils in the spatially heterogeneous treatments (i.e. a Graeco-Latin square for the fine-grained heterogeneous treatment). The whole setup was replicated three times, using the three independent soil replicates. In total there were 42 containers in the test phase (7 soil treatments $\mathrm{x} 2$ plant communities $\mathrm{x} 3$ replicates).

Each container was filled with $2.5 \mathrm{~kg}$ sterilized gravel (quartz, $4-8 \mathrm{~mm}$ ) and then with $8 \mathrm{~kg}$ of conditioned soil ( $500 \mathrm{~g}$ per gridcell). For each treatment the containers were filled with conditioned soil in the same way: weighing $500 \mathrm{~g}$ of the appropriate conditioned soil type and then carefully pouring the soil into the respective gridcell and continuing until all cells of the container were filled. Each container was planted with 32 seedlings, planting two individuals of the same species into each gridcell (each seedling $1 \mathrm{~cm}$ from the gridcell midpoint). The experimental design ensured that all plant species were grown on all soils in the heterogeneous treatments. Seeds were germinated in the same way as in the conditioning phase. Seedlings that died upon transplantation were replaced once during the first week. The containers were placed in the greenhouse in a complete randomized design under the same conditions as during the conditioning phase and allowed to grow for eight weeks. The soil was kept moist by regular watering ( 2 or 3 times per week depending on evapotranspiration rates). After 8 weeks of growth, aboveground plant biomass was clipped flush with the soil, dried $\left(72{ }^{\circ} \mathrm{C}, 48 \mathrm{~h}\right)$, and weighed separately per gridcell for each of the containers (i.e. 16 observations per container, with known locations of each observation within the container).

\section{Data analysis}

We calculated the evenness index ( $\mathrm{J}$ ') based on the shoot biomass of each species present in each container as a measure of plant diversity (Pielou 1966). Both plant diversity and total plant biomass in each container were analysed with simple fixed effects models including plant mix, spatial conditioning treatment (four levels: 1) uniform mono-conditioned; 2) uniform mixed-conditioned; 3) fine-grained heterogeneous; and 4) coarse-grained heterogeneous) and their interaction in the model. Shifts in competitive hierarchies were analysed using relative abundance of each species per container. These data were analysed per community using linear mixed models (LMM) with container as random factor. Test plant species, 
conditioned soil and level of spatial heterogeneity and their interactions were included as fixed factors.

To examine how PSF effects change with spatial heterogeneity we analysed differences in plant biomass on gridcell level using LMMs. The uniform mixedconditioned soil treatment was excluded from this analysis as in this treatment the soil effects on plant performance could not be attributed to individual conditioning species. These models included random effects for container and gridcell. The gridcell factor was introduced to account for positional effect within containers, but given the rotational symmetry in the within container design the gridcell factor had three levels (corner (4 per container), edge (8), or centre (4) of the container). Test plant species, conditioned soil and level of spatial heterogeneity (uniform mono-conditioned, heterogeneous fine, and heterogeneous course) and their interactions were included as fixed factors and analyses were conducted separately for the two plant communities. We constructed separate own-foreign soil planned contrasts for each species at each level of spatial heterogeneity to test for direct PSF (Adbi and Williams 2010, Brinkman et al. 2010).

Differences in abiotic conditions and shoot biomass at the end of the conditioning phase were tested with one-way ANOVA's. Spearman correlations were used to assess the relationship of the soil abiotic variables at the end of the conditioning phase and the shoot biomass at the end of the test phase. These correlations were calculated for each plant community separately using all uniform soils that correspond with the plant community $(n=15)$. Differences between soils in fungal community composition (T-RFLP data) were visualized using nonmetric multidimensional scaling (NMDS) and tested using a multiple response permutation procedure (MRPP). Prior to analysis we removed singleton loci from the T-RFLP data.

All analyses were conducted in R v.3.3.0 (R Core Team, 2016) and model assumptions were checked graphically. Heteroscedasticity was modelled using generalized least squares (Pinheiro \& Bates, 2000; Zuur et al., 2009). Linear mixed models were fitted with NLME v.3.1-128 (Pinheiro et al., 2016) and posthoc comparisons were conducted in LSMEANS v.2.23 (Lenth 2015). Type I errors of multiple comparisons were controlled using the false discovery rate (Benjamini and Hochberg 1995). Planned own-foreign contrasts were analysed within the MULTCOMP v.1.4-5 package (Hothorn et al., 2008). 


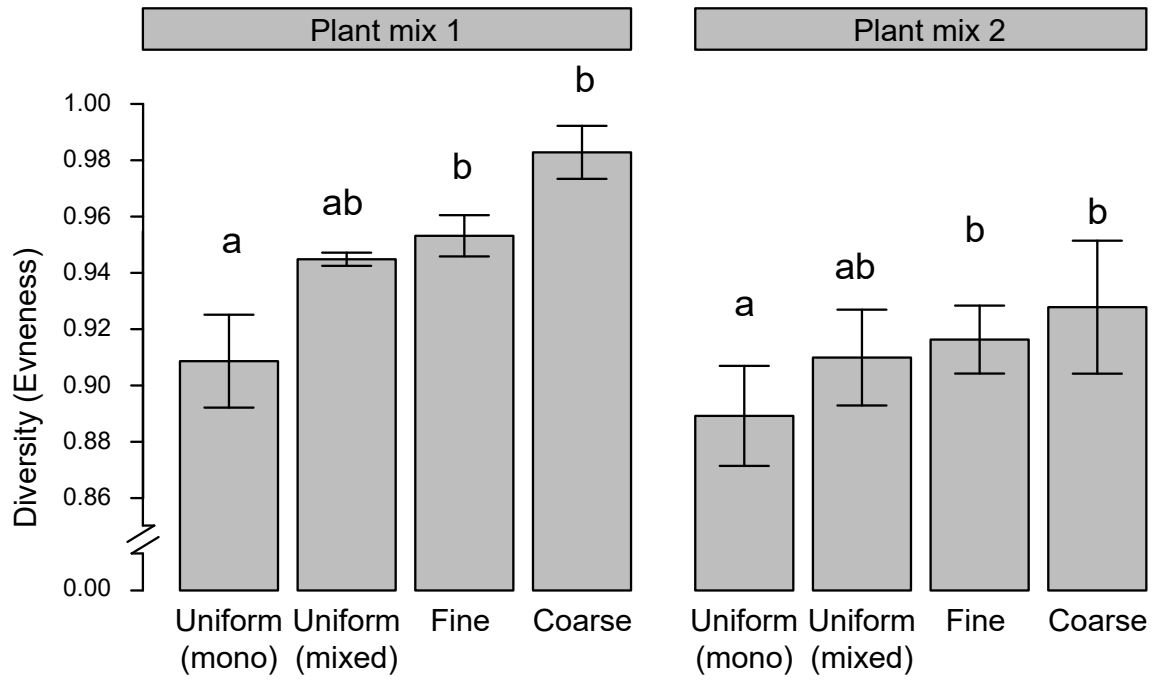

Fig. 6.2. Plant evenness (mean $\pm \mathrm{SE}$ ) in the four spatial PSF heterogeneity treatments for both plant mixtures.

Soils were either spatially homogeneous (Uniform) or heterogeneous (Fine or Coarse grained) and conditioned by either one (mono) or four (mixed, Fine \& Coarse) species. Different letters indicate significant differences among bars (Table S6.2).

\section{Results}

Plant evenness in the mixtures was higher when soils were distributed heterogeneously in space compared to the uniform mono-conditioned treatment (Fig. 6.2; Table S6.2). Interestingly, while heterogeneous soils led to higher diversity than the uniform mono-conditioned soils, uniform soils that were conditioned simultaneously by four species, i.e. mixed-conditioned soils, also had high levels of plant diversity (Fig. 6.2).

On mono-conditioned uniform soils, heterospecific species often outperformed the focal species which self-conditioned the soil (Fig. 6.3; Table S6.3). This led to altered competitive hierarchies where different plant species gained dominance across the different mono-conditioned spatially uniform soils (Fig. 6.3). However, $H$. radicata (Mix 1) and F. rubra (Mix 2) were exceptions to this, as they were still competitively superior on their own self-conditioned soil. For F. rubra this was true even though this species had a significant negative direct PSF (Table S6.4). In heterogeneous soils, the performance of all species was always intermediate to 
the best and the worst performance that was observed across the uniform monoconditioned soils (Fig. 6.3; Table S6.3).

In uniform mono-conditioned soils five out of six species experienced significant negative PSF in the mixed plant communities (Table S6.4b). Grasses performed worst on grass-conditioned soil and better on dicot soil (Fig. 6.4a,b; Table S6.4), while dicots typically showed the reverse pattern, although they also performed well on unrelated dicot-conditioned soils (e.g. J. vulgaris grown in L. corniculatus soil performed better than on $\mathrm{H}$. radicata-soil; Fig. 6.4a,b). In the spatially heterogeneous soils these patterns were altered and grasses sometimes had the

a) Plant mix 1

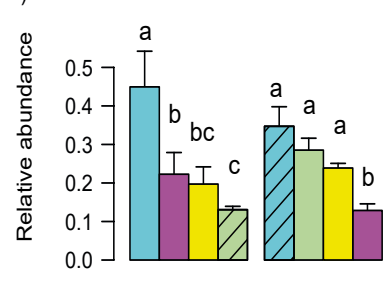

Soils: a

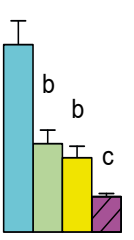

Jv Uniform

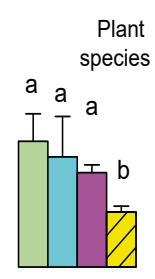

LC Uniform

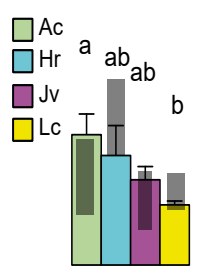

Mix Uniform

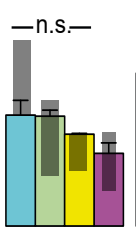

Mix Coarse

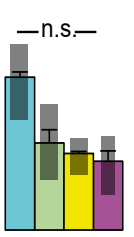

Mix Fine b) Plant mix 2

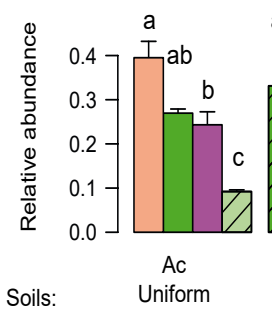

Space:

Conditioning a
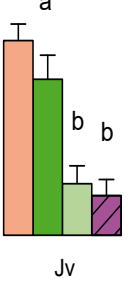

Uniform
Plant $\square$ Ac

a species $\square \mathrm{Fr}$ a a

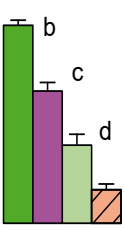

$\mathrm{Tp}$

Uniform

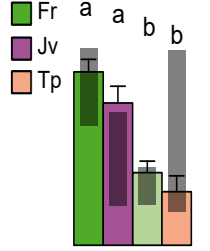

Mix

Uniform

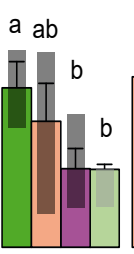

a

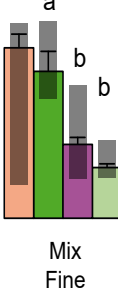

Coarse

homogeneous

heterogeneous

Fig. 6.3. Competitive hierarchies across different conditioned soils for two plant mixtures.

Relative abundance (mean $\pm \mathrm{SE}$ ) of the four plant species per treatment is shown and the species are ranked from high to low abundance. Different letters indicate significant differences among bars tested per soil treatment. Hatched bars indicated that plant species in the mixture grew on self-conditioned soil. The grey shading in the mixed conditioned soil treatments (the right three sets of bars) indicates the highest to lowest range for each focal species on the four uniform monoconditioned soils (left four sets of bars). In all cases the relative abundance of the focal species was not significantly different from this range. For abbreviations see Fig. 6.1. 
highest biomass on grass-conditioned or even self-conditioned soils (e.g. F. rubra on A. capillaris soil in Coarse and F. rubra soil in Fine; Fig. 6.4d,f). Similarly, forbs did not necessarily perform best on grass-conditioned soils (Fig. 6.4) in the heterogeneous treatments. In general, direct PSFs, quantified as own-foreign contrasts, became less strong and non-significant in spatially heterogeneous soils (Fig. 6.4; Table S6.4b). Only F. rubra (Mix 2) and J. vulgaris (Mix 1) in Coarse and T. pratense (Mix 2) in Fine did experience significant negative direct PSF in heterogeneous soils (Table S6.4b).

Total biomass in the mixed plant communities was not affected by the spatial heterogeneity treatments (Table S6.5). Soil conditioning altered soil nitrogen and acidity (Table S6.1), but total biomass in the test phase was not related to any of
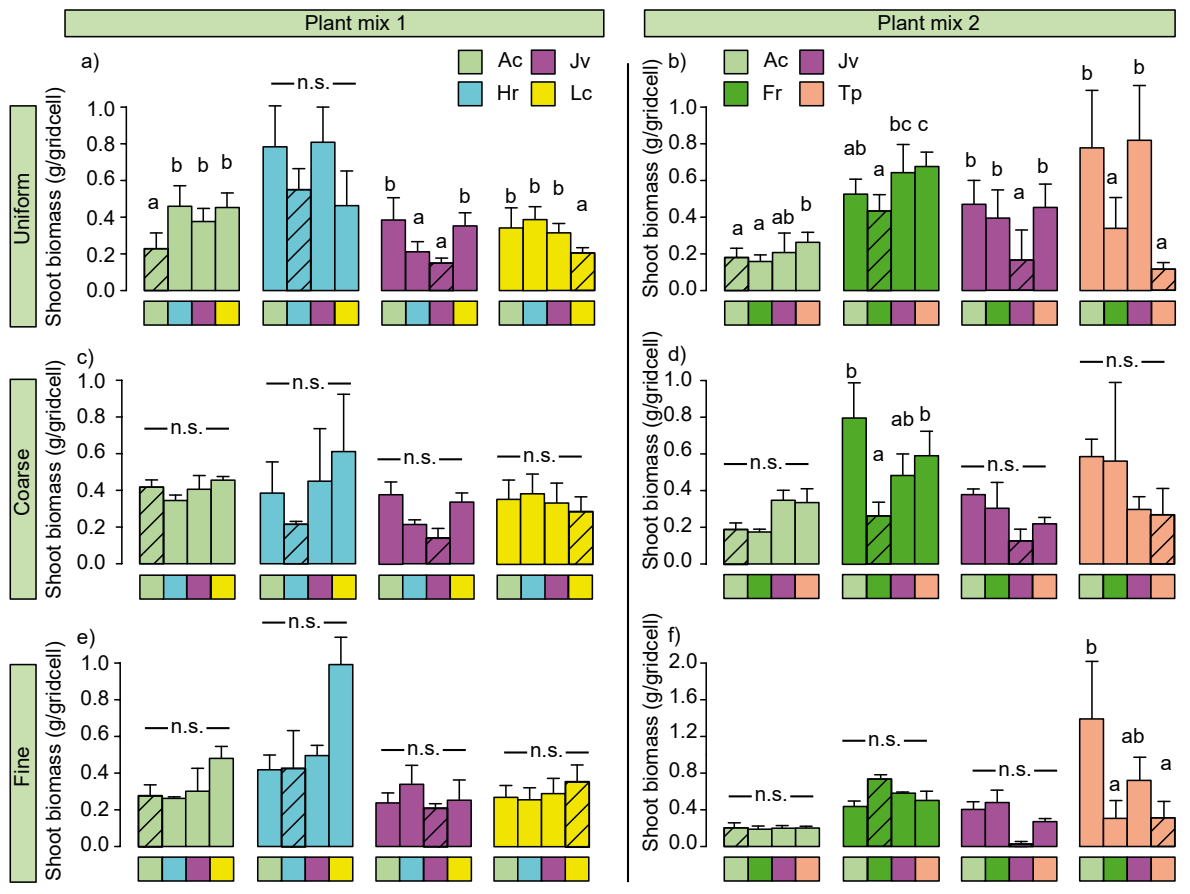

Fig. 6.4. Shoot biomass (mean $\pm S E$ ) per species in two plant mixtures in mono-conditioned uniform $(a, b)$ and two heterogeneous, coarse- $(c, d)$ and fine-grained $(e, f)$, PSF treatments.

Shown are all four plant species (coloured bars) per community on all four conditioned soils (coloured squares below bars) in that mix (Fig. 6.1). Hatched bars indicated that plants grew on self-conditioned soil. Different letters indicate significant differences among bars and were tested separately per species and level of spatial heterogeneity. For abbreviations see Fig. 6.1. 
the abiotic variables or to shoot biomass in the conditioning phase (Table S6.6). Soil conditioning did lead to clear differences in fungal community composition among the six plant species; conditioning by grasses, in particular, led to fungal communities that were different from the communities created by forbs or legumes (Fig S6.1, MRPP F = 1.57, $\mathrm{p}=0.002$ ).

\section{Discussion}

6 In our study we compared the PSF effects of monospecific soil conditioning and multispecies conditioning on plant diversity. Our results show that it is not the spatial heterogeneity in PSFs, but rather the diversity of plant species that conditioned the soil that promotes plant alpha diversity. When the soil had been conditioned by all members of the experimental community, plant diversity was higher than when only a single species conditioned the soil. In addition, there was no difference in evenness among the spatially heterogeneous treatments. Plantsoil feedbacks, driven by e.g. soil-borne pathogenic fungi and nematodes, have been suggested to maintain plant diversity at small spatial scales (e.g. (Petermann et al. 2008, De Kroon et al. 2012, Bever et al. 2015). Our data now adds to this by showing that the diversity of the plant species conditioning the soil drives the diversity of the plant community subsequently growing in that soil, through plant-soil feedback.

A long-standing alternative explanation for plant alpha diversity has been that spatially heterogeneous abiotic conditions, such as soil nutrient availability, create niches that can be occupied by different species (Tilman 1982, Harpole et al. 2016). However, meta-analyses testing the effects of spatial heterogeneity in abiotic conditions on plant diversity have shown that abiotic heterogeneity only has a positive effect on alpha diversity when the scale of abiotic heterogeneity exceeds the reach of interacting plants (sensu Casper et al. 2003). When the heterogeneity is more fine-grained $(<20 \times 20 \mathrm{~cm}$ on average) the effect on diversity is negative (Lundholm 2009, Tamme et al. 2010), because heterogeneity then selects plant species that are efficient root foragers (Gazol et al. 2013). Hence abiotic heterogeneity cannot explain high plant diversity at this scale, e.g. up to 42 species were recorded at 20x20 cm in an Estonian wooded meadow (Kull and Zobel 1991, Wilson et al. 2012).

The difference in spatial grain (Fine vs. Coarse) of PSF heterogeneity did not affect plant performance in mixtures, and did not alter plant diversity at a scale of $22 \times 26 \mathrm{~cm}$. Furthermore, when four species conditioned the soil simultaneously 
(mixed-conditioned), plant diversity was similar to the spatially heterogeneous PSF treatments although not significantly different from the mono-conditioned uniform treatment. In line with our results, Hendriks et al. (2013) showed that mixing own and foreign soil releases plants from their negative self-feedback. Models of PSF-mediated plant coexistence suggest that PSF effects need to be highly localized to maintain diversity (Bonanomi et al. 2005, Fukami and Nakajima 2013, Mack and Bever 2014, Abbott et al. 2015). Empirical work on microbes corroborates this by showing that coexistence only occurs in communities with localized dynamics (Kerr et al. 2002). Our study was carried out at a scale where the roots of all plant individuals could in theory forage the entire experimental unit and be in contact with all soils independent of the spatial configuration. Therefore, our results suggest that as long as multiple species conditioned the soil within the plant roots' zone of influence (Casper et al. 2003), the exact spatial pattern of conditioning does not matter.

We found that in uniform mono-conditioned soils direct PSFs were negative and strong enough to alter competitive hierarchies (Shannon et al. 2012, Hendriks et al. 2013, Pendergast et al. 2013, Jing et al. 2015), and heterospecifics became most abundant. In soils conditioned by multiple species (both heterogeneous and mixed-conditioned uniform soils) PSF effects were less pronounced and this led to more equalized competitive abilities among species and higher diversity. Together this underscores the idea that PSFs, through their density dependent effects on plant performance, may mediate competitive intransitivity among species (De Kroon et al. 2012; Soliveres et al. 2015; De Kroon \& Jongejans 2016), which is thought to influence dynamics of many natural communities (Soliveres et al. 2015). As a next step, pairwise competition experiments among all species in the community are needed to definitively demonstrate PSF-mediated intransitivity of competitive abilities (Petraitis 1979, Laird and Schamp 2006, Jing et al. 2015).

We found strong direct PSF effects for most plant species, as was found in other studies (Kardol et al. 2007, Petermann et al. 2008, Kulmatiski et al. 2008). However, PSFs were not strong enough to prevent all species from becoming competitively dominant in their own self-conditioned soils. Both F. rubra and $H$. radicata were the most abundant species in the mixed communities on their own soils, even though both species had a better performance on heterospecificconditioned soils (significant for F. rubra). We did not use seed from the field where the soil was collected, except for $J$. vulgaris, so perhaps the soil community was naïve to these genotypes at the start of the experiment and PSF may be 
stronger with co-adapted plant genotypes and soil communities (Felker-Quinn et al. 2011, Veen et al. 2014). In addition, PSF may also affect plant germination and establishment (Grubb 1977, Brandt et al. 2013, Burns and Brandt 2014), which we did not test as the experiment started from seedlings. Thus, PSF effects could be stronger in the field.

Field observations show that the spatiotemporal patterns of species replacement in late-successional grasslands is consistent with the model of PSF mediated coexistence (Van der Maarel and Sykes 1993, Herben et al. 1993, De Kroon and Jongejans 2016), suggesting that the same mechanisms may be in operation. However, an open question is how plants condition soils spatially in the field. We know that different plants in natural communities have distinct soil communities (Bezemer et al. 2010), but how they build up over the life-time of a plant (Kardol et al. 2013, Zhang et al. 2016) and whether these induce the same PSF effects as in the greenhouse is unclear. It will be key to investigate how important PSFs are in driving plant community composition in relation to e.g. the colonization-competition trade-off (Tilman 1994), as well as in interaction with large herbivores (Chesson and Kuang 2008, Veen et al. 2014) and across abiotic gradients (Bever et al. 2015).

Plant-soil feedbacks can be mediated both abiotically and by the soil community (Ehrenfeld et al. 2005). While it was not possible in this experiment to tease out the exact causes of feedback for each plant species, we did find clear changes in fungal community composition due to soil conditioning. In addition, differences in soil abiotic conditions did not correlate with measures of plant performance and there were no differences in total biomass among the spatial treatments. In combination with the observation that the grain of heterogeneity did not affect plant performance, we hypothesize that changes in soil community composition, e.g. in soil fungi, affected plant performance as was shown in other studies (Bradley et al. 2008, Hendriks et al. 2013, Bever et al. 2015).

In conclusion, we show that PSFs can maintain plant diversity when multiple species condition the soil, but that at small scales this does not depend on the spatial distribution of PSFs. The presence of soil conditioned by all plant species in the community equalizes competitive differences among plants relative to soil conditioned by one species. The spatial grain of PSF heterogeneity had no effect, suggesting that it is the presence, in sufficient amount, of each species' soil-borne antagonists that leads to coexistence. Future studies are needed to quantify the 
importance of PSF in the field relative to interacting factors such as herbivory, across soil abiotic conditions and successional stage. 


\section{Supplementary information}

\section{Method S6.1 -Protocol for T-RFLP analyses of conditioned soils.}

Terminal restriction fragment length polymorphisms analysis (T-RFLP) was used to assess differences in the composition of soil fungal communities among the 18 mono-conditioned soil samples. Total DNA was extracted from $0.25 \mathrm{~g}$ frozen soil $\left(-20^{\circ} \mathrm{C}\right)$ per sample with a Power Soil DNA isolation kit (MOBIO laboratories, Carlsbad, CA, USA) using a bead beating system. DNA concentration was measured using the NanoDrop 2000. For the samples with a DNA concentration below $10 \mathrm{ng} / \mu \mathrm{L}$ a new DNA-extraction was performed. The ITS region of the fungal rDNA was amplified by PCR using the primers ITS1F (White et al. 1990) and ITS4 (Gardes and Bruns 1993), dual-end labelled with 6FAM and NED respectively. The PCR reaction contained $13.8 \mu \mathrm{L}$ milli-Q water, $2.5 \mu \mathrm{L}$ 10x Fast Start High Fidelity Reaction Buffer (Roche Diagnostics, Almere, The Netherlands), $2.5 \mu \mathrm{L}$ dNTP mix (2 mM of each), $2.5 \mu \mathrm{L}$ ITS1Ff-6FAM primer $(10 \mu \mathrm{M}), 2.5 \mu \mathrm{L}$ ITS4r-NED primer $(0.2 \mu \mathrm{M}), 0.2 \mu \mathrm{L}$ Fast Start High Fidelity Enzyme Blend ( $5 \mathrm{U}$ $\mu \mathrm{L}^{-1}$; Roche Diagnostics) and $1 \mu \mathrm{L}$ template DNA. PCR cycle conditions were 5 min at $95^{\circ} \mathrm{C}, 34$ cycles of $30 \mathrm{~s}$ at $95^{\circ} \mathrm{C}, 40 \mathrm{~s}$ at $55^{\circ} \mathrm{C}$ and 1 min at $72^{\circ} \mathrm{C}$, followed by $10 \mathrm{~min}$ at $72^{\circ} \mathrm{C}$ before cooling. PCR product presence and quality were verified on $1.5 \%$ agarose gels prior to restriction digestion. Two restriction enzymes, HhaI and Taq $\alpha$ (New England Biolabs, Ipswich, MA, USA), were used to digest the dual end-labelled DNA amplicons in a mixture containing $3.5 \mu \mathrm{L}$ ddH2O, $1 \mu \mathrm{L}$ buffer, $0.1 \mu \mathrm{L}$ Bovine Serum Albumin, $5 \mu \mathrm{L}$ PCR product and $0.4 \mu \mathrm{L}$ restriction enzyme, incubated at $37{ }^{\circ} \mathrm{C}$ ( $\mathrm{HhaI}$ ) or at $65^{\circ} \mathrm{C}$ (Taq $\left.\alpha \mathrm{I}\right)$ for $3 \mathrm{~h}$, followed by inactivation at $80^{\circ} \mathrm{C}$ for $20 \mathrm{~min}$. Restriction products were purified using ethanol precipitation. Fragment length polymorphism analysis was performed on an automated 3130 Genetic Analyser sequencer, using GeneScan-500 LIZ (Applied Biosystems, Carlsbad, CA, USA) as the internal lane size standard. Samples which were over- (highest peak $>80000$ relative fluorescence units, rfu) or under-loaded (highest peak $<1000 \mathrm{rfu}$ ) were re-run with an adjusted concentration. Peaks at sizes $<50$ base pairs (bp) and $>500$ bp were removed. Peaks were aligned to terminal restriction fragments (TRFs) among the samples by applying a clustering threshold of $0.5 \mathrm{bp}$. All peaks higher than $0.3 \%$ of the sum of all peaks in a sample were included in the analysis (Bezemer et al. 2013). In total 544 fungal TRFs were detected in the soils, of which 385 were not singletons. 
Table S6.1. Soil chemistry and aboveground plant biomass of each conditioning treatment after eight weeks of conditioning the soil (phase 1).

Mean $( \pm \mathrm{SE})$ values and results of one-way ANOVA analyses of differences among treatments are shown (different letters indicate significant differences the treatments). AGB P1 = aboveground biomass in phase $1, \mathrm{SOM}=$ soil organic matter, ns $=$ not significant, ${ }^{*} P<0.05,{ }^{* * *} P<0.001$.

\begin{tabular}{lcccccc}
\hline Conditioning & $\begin{array}{c}\mathrm{PO}_{4}-\mathrm{P}^{*} \\
(\mathrm{mg} / \mathrm{kg})\end{array}$ & $\begin{array}{c}\mathrm{NO}_{3}-\mathrm{N} \\
(\mathrm{mg} / \mathrm{kg})\end{array}$ & $\begin{array}{c}\mathrm{NH}_{4}-\mathrm{N} \\
(\mathrm{mg} / \mathrm{kg})\end{array}$ & $\begin{array}{c}\text { Acidity } \\
(\mathrm{pH})\end{array}$ & $\begin{array}{c}\mathrm{SOM} \\
(\% \mathrm{w}: \mathrm{w})\end{array}$ & $\begin{array}{c}\text { AGB P1 } \\
\left(\mathrm{g} \mathrm{pot}^{-1}\right)\end{array}$ \\
\hline treatment & & & & & & \\
\hline A. capillaris & 95.50 & $3.94^{\mathrm{a}}$ & 6.24 & $5.98^{\mathrm{ab}}$ & 3.20 & $4.00^{\mathrm{de}}$ \\
& $(1.47)$ & $(0.28)$ & $(0.61)$ & $(0.06)$ & $(0.07)$ & $(0.08)$ \\
F. rubra & 97.06 & $1.76^{\mathrm{bc}}$ & 4.19 & $5.90^{\mathrm{ab}}$ & 3.20 & $3.52^{\mathrm{e}}$ \\
& $(1.19)$ & $(0.69)$ & $(0.70)$ & $(0.03)$ & $(0.09)$ & $(0.07)$ \\
H. radicata & 100.47 & $1.03^{\mathrm{c}}$ & 6.26 & $5.89^{\mathrm{ab}}$ & 3.15 & $3.97^{\mathrm{de}}$ \\
& $(0.73)$ & $(0.09)$ & $(0.28)$ & $(0.02)$ & $(0.03)$ & $(0.09)$ \\
J. vulgaris & 100.30 & $0.81^{\mathrm{c}}$ & 5.67 & $5.99^{\mathrm{a}}$ & 3.32 & $2.52^{\mathrm{f}}$ \\
& $(5.31)$ & $(0.14)$ & $(0.78)$ & $(0.03)$ & $(0.01)$ & $(0.05)$ \\
L. corniculatus & 103.70 & $3.40^{\mathrm{ab}}$ & 6.21 & $5.89^{\mathrm{ab}}$ & 3.05 & $8.39^{\mathrm{b}}$ \\
& $(0.78)$ & $(0.54)$ & $(0.71)$ & $(0.03)$ & $(0.16)$ & $(0.22)$ \\
T. pratense & 98.70 & $0.92^{\mathrm{c}}$ & 7.05 & $5.82^{\mathrm{b}}$ & 3.15 & $10.87^{\mathrm{a}}$ \\
& $(4.52)$ & $(0.17)$ & $(0.22)$ & $(0.01)$ & $(0.24)$ & $(0.33)$ \\
Mix 1 & 103.2 & $1.67^{\mathrm{bc}}$ & 6.20 & $5.89^{\mathrm{ab}}$ & 3.30 & $4.62 \mathrm{~d}$ \\
& $(5.05)$ & $(0.49)$ & $(0.92)$ & $(0.03)$ & $(0.25)$ & $(0.30)$ \\
Mix 2 & 106.6 & $1.55^{\mathrm{bc}}$ & 5.81 & $5.89^{\mathrm{ab}}$ & 3.32 & $5.87 \mathrm{c}$ \\
& $(4.65)$ & $(0.49)$ & $(0.82)$ & $(0.01)$ & $(0.23)$ & $(0.32$ \\
& & & & & & \\
\hline & & $7.40^{* * *}$ & $1.49^{\mathrm{ns}}$ & $2.80^{*}$ & $0.36^{\mathrm{ns}}$ & $241.53^{* * *}$ \\
& & & & & & \\
\hline
\end{tabular}

${ }^{*} \mathrm{P}-\mathrm{Olsen}$ extraction, ${ }^{\dagger} \mathrm{H}_{2} \mathrm{O}$ extraction 
Table S6.2. Results of statistical analyses of plant evenness.

Two analyses were performed, one including the mixed-conditioned uniform treatment (4 spatial treatments), and one excluding it (3 spatial treatments).

\begin{tabular}{lcccccccc}
\hline & \multicolumn{3}{c}{ 4 spatial treatments } & & \multicolumn{3}{c}{3 spatial treatments } \\
\cline { 2 - 4 } \cline { 7 - 9 } Terms & d.f. & F & p-value & & d.f & F & p-value \\
\hline Plant mix $(\mathrm{PM})$ & 1,34 & 15.23 & $\mathbf{0 . 0 0 0 4}$ & & 1,30 & 11.11 & $\mathbf{0 . 0 0 2}$ \\
Spatial heterogeneity $(\mathrm{SH})$ & 3,34 & 3.69 & $\mathbf{0 . 0 2}$ & & 2,30 & 5.47 & $\mathbf{0 . 0 0 9}$ \\
PM x SH & 3,34 & 0.34 & 0.79 & & 2,30 & 0.51 & 0.60 \\
\hline
\end{tabular}

Table S6.3. Results for LMM analyses on plant relative abundances in both plant mixtures separately.

\begin{tabular}{lccccccc}
\hline & \multicolumn{3}{c}{ Mix 1 } & & \multicolumn{3}{c}{ Mix 2 } \\
\cline { 2 - 4 } \cline { 6 - 8 } Terms & d.f. & F & p-value & & d.f & F & p-value \\
\hline Plant species (PS) & 3,42 & 26.68 & $<\mathbf{0 . 0 0 0 1}$ & & 3,42 & 35.45 & $<\mathbf{0 . 0 0 0 1}$ \\
Soil treatment (ST) & 6,14 & 5.00 & $\mathbf{0 . 0 0 6 2}$ & & 6,14 & 1.77 & 0.18 \\
PS x ST & 18,42 & 4.10 & $\mathbf{0 . 0 0 0 1}$ & & 18,42 & 7.66 & $<\mathbf{0 . 0 0 0 1}$ \\
\hline
\end{tabular}


Table S6.4. Linear mixed model analysis of shoot biomass in two mixtures of plant species at gridcell level.

a) Results of the overall analyses, b) results of planned Own-Foreign soil contrasts (i.e. direct PSF) for each species in each of the three levels of spatial heterogeneity. Negative values indicate negative feedback.

\begin{tabular}{lccccccc}
\hline a) & & \multicolumn{2}{c}{ Mix 1 } & & \multicolumn{2}{c}{ Mix 2 } \\
\cline { 8 - 9 } \cline { 7 - 8 } Terms & d.f. & & F & p-value & & F & P-value \\
\hline Plant species (PS) & 3,189 & 47.23 & $<\mathbf{0 . 0 0 0 1}$ & & 88.47 & $<\mathbf{0 . 0 0 0 1}$ \\
Conditioned soil (CS) & 3,189 & 3.49 & $\mathbf{0 . 0 2}$ & & 4.58 & $\mathbf{0 . 0 0 4}$ \\
Spatial heterogeneity (SH) & 2,15 & 0.22 & 0.81 & & 0.20 & 0.82 \\
PS x CS & 9,189 & 7.80 & $<\mathbf{0 . 0 0 0 1}$ & & 8.24 & $<\mathbf{0 . 0 0 0 1}$ \\
PS x SH & 6,189 & 2.11 & 0.05 & & 1.73 & 0.12 \\
CS x SH & 6,189 & 1.00 & 0.42 & & 1.68 & 0.13 \\
PS x CS x SH & 18,189 & 1.77 & $\mathbf{0 . 0 3}$ & & 1.82 & $\mathbf{0 . 0 3}$ \\
\hline
\end{tabular}

b)

Mix 1

Spatial

heterogeneity

Uniform

(mono-

conditioned)

Lc

species

Ac

$\mathrm{Hr}$

Jv

$-5.12$

$-3.83$

\begin{tabular}{ccc}
\multicolumn{3}{c}{ Mix 1 } \\
\hline Plant & Z & p-value
\end{tabular}

Ac

$\mathrm{Hr}$

Jv $\quad-2.91$

Lc $\quad-1.02$

0.17

\begin{tabular}{lll}
\hline Fine & Ac & -0.77 \\
& Hr & -1.22 \\
& Jv & -1.15 \\
& Lc & 1.22 \\
\hline
\end{tabular}

\begin{tabular}{lll}
\hline Fine & Ac & -0.77 \\
& $\mathrm{Hr}$ & -1.22 \\
& $\mathrm{Jv}$ & -1.15 \\
& $\mathrm{Lc}$ & 1.22 \\
\hline
\end{tabular}

$\mathrm{Hr}$

Jv

$-1.22$

$-1.15$

1.22

Mix 2

\begin{tabular}{ccc}
\multicolumn{3}{c}{ Mix 2 } \\
\hline $\begin{array}{c}\text { Plant } \\
\text { species }\end{array}$ & $\mathrm{Z}$ & $\mathrm{p}$-value \\
\hline Ac & -0.95 & 0.38 \\
$\mathrm{Fr}$ & -3.44 & $\mathbf{0 . 0 0 2}$ \\
$\mathrm{Jv}$ & -3.49 & $\mathbf{0 . 0 0 2}$ \\
$\mathrm{Tp}$ & -5.60 & $<\mathbf{0 . 0 0 0 1}$ \\
\hline Ac & -1.52 & 0.19 \\
$\mathrm{Fr}$ & -3.44 & $\mathbf{0 . 0 0 2}$ \\
$\mathrm{Jv}$ & -1.09 & 0.33 \\
$\mathrm{Tp}$ & -1.12 & 0.33 \\
$\mathrm{Ac}$ & 0.12 & 0.91 \\
$\mathrm{Fr}$ & 2.16 & 0.05 \\
$\mathrm{Jv}$ & -2.23 & 0.05 \\
$\mathrm{Tp}$ & -2.60 & $\mathbf{0 . 0 2}$ \\
\hline
\end{tabular}


Table S6.5. Results of statistical analyses of total shoot biomass per container.

Two analyses were performed, one including the mixed-conditioned uniform treatment (4 spatial treatments), and one excluding it (3 spatial treatments).

\begin{tabular}{|c|c|c|c|c|c|c|}
\hline \multirow[b]{2}{*}{ Terms } & \multicolumn{3}{|c|}{4 spatial treatments } & \multicolumn{3}{|c|}{3 spatial treatments } \\
\hline & d.f. & $\mathrm{F}$ & $\mathrm{p}$-value & d.f & $\mathrm{F}$ & p-value \\
\hline Plant mix (PM) & 1,34 & 1.40 & 0.25 & 1,30 & 0.77 & 0.39 \\
\hline Spatial heterogeneity $(\mathrm{SH})$ & 3,34 & 1.30 & 0.29 & 2,30 & 1.09 & 0.35 \\
\hline PM x SH & 3,34 & 0.44 & 0.73 & 2,30 & 0.48 & 0.62 \\
\hline
\end{tabular}

Table S6.6. Spearman's rho correlation coefficients for the relationship of total shoot biomass of the tested plant mixtures and measured abiotic conditions of the soil and shoot biomass after soil conditioning (i.e. at the end of phase 1).

None of the correlations $(\mathrm{n}=15)$ were significant (i.e. $\mathrm{p}>0.05)$.

\begin{tabular}{lcccccc}
\hline Species mixture & $\mathrm{PO}_{4}$ & $\mathrm{NO}_{3}$ & $\mathrm{NH}_{4}$ & Acidity & $\begin{array}{c}\text { Soil } \\
\text { organic } \\
\text { matter }\end{array}$ & $\begin{array}{c}\text { Phase } \\
1 \text { shoot } \\
\text { biomass }\end{array}$ \\
\hline Mix 1 & -0.38 & 0.04 & 0.48 & -0.19 & -0.19 & -0.15 \\
Mix 2 & -0.01 & 0.34 & 0.32 & 0.43 & 0.25 & -0.15 \\
\hline
\end{tabular}




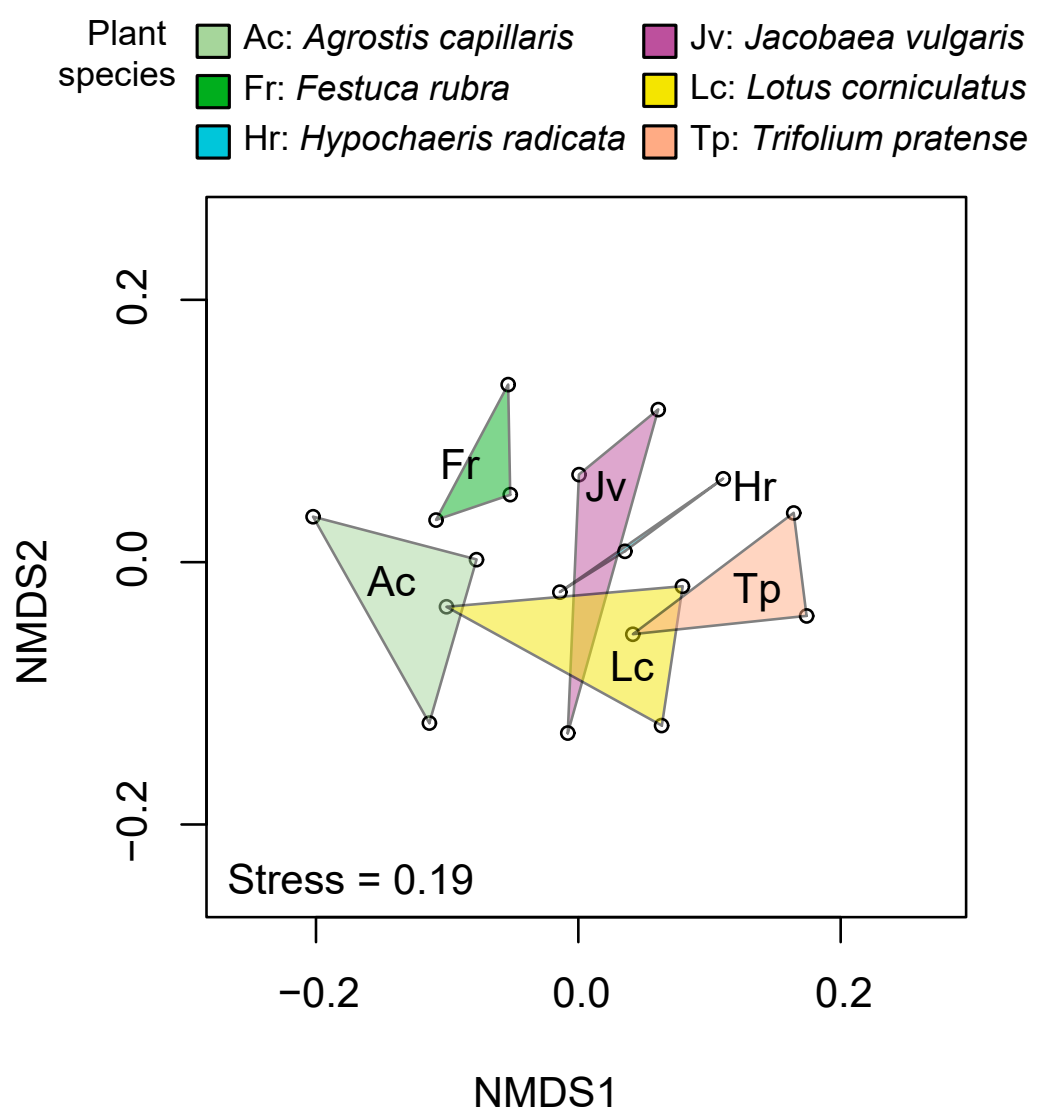

Fig. S6.1. NMDS visualization of the soil fungal community composition as revealed by T-RFLP.

The three replicates (circles) of each conditioned soil are connected by lines. Samples that are closer together are more similar then samples that are further apart. 


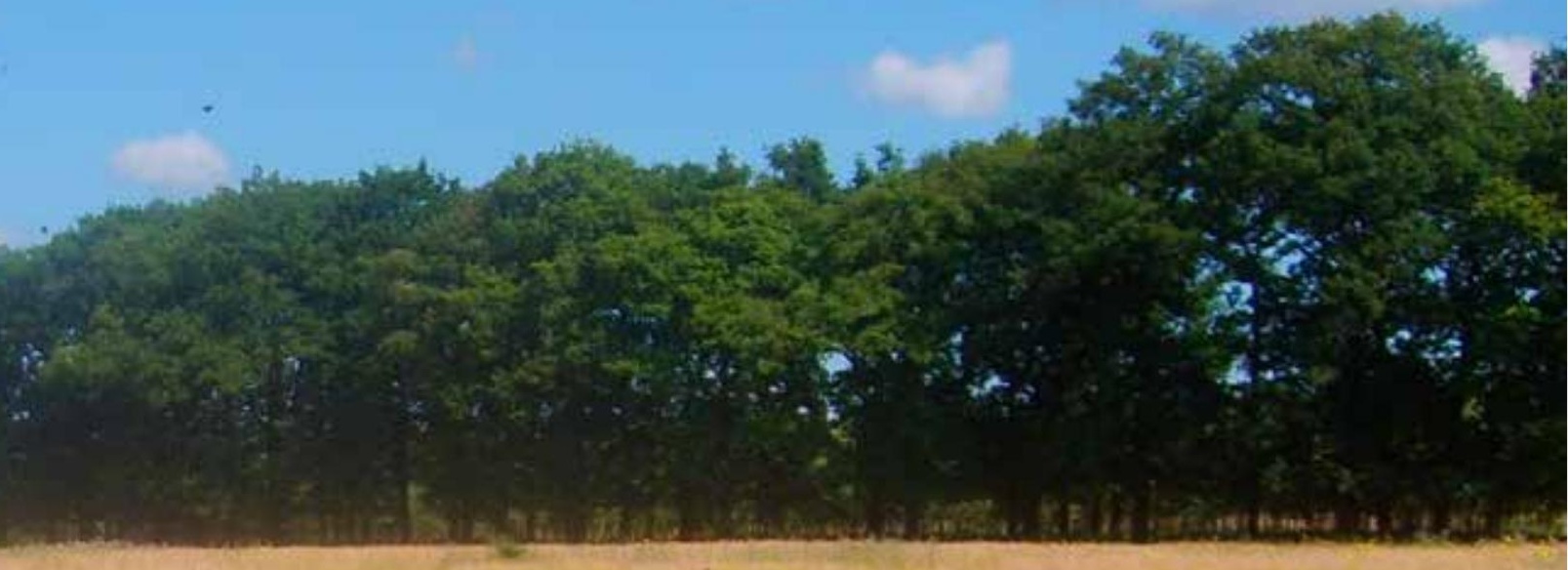

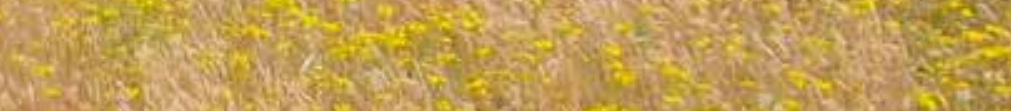

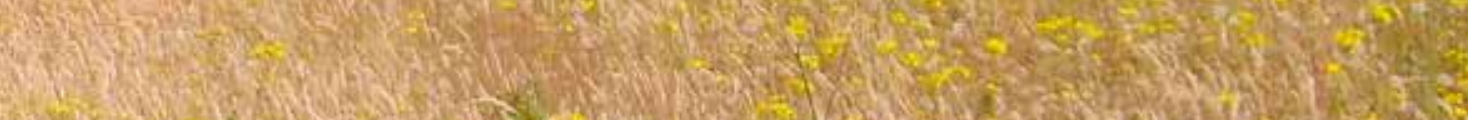

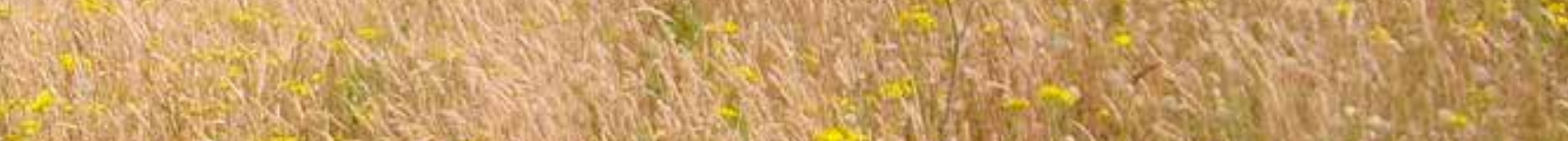
at

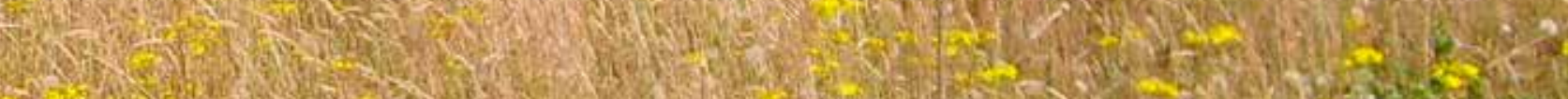

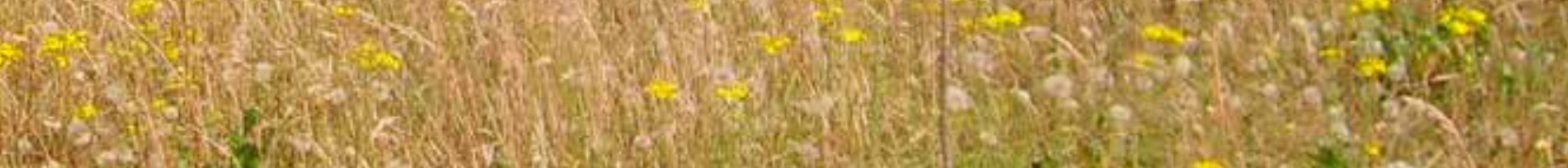

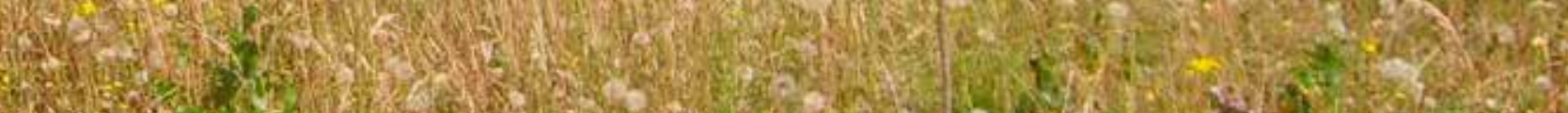

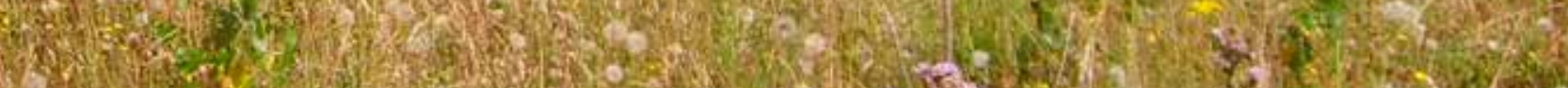
1.

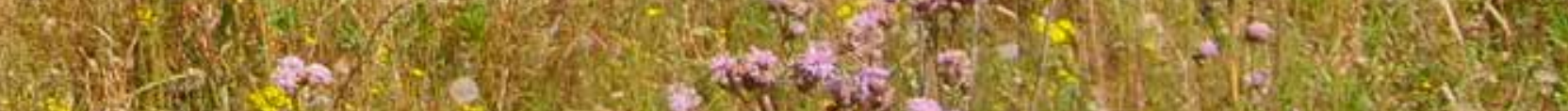

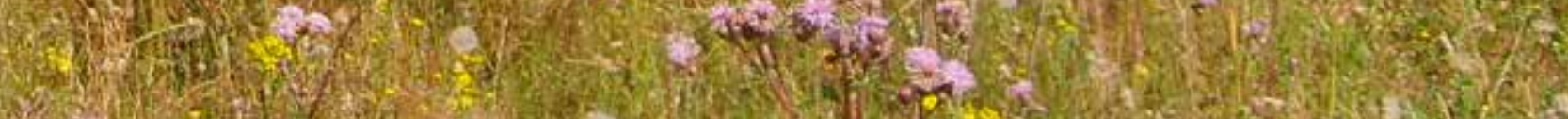

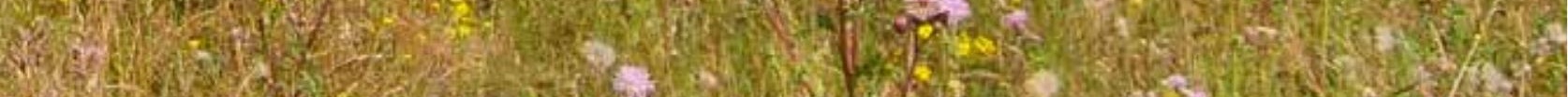

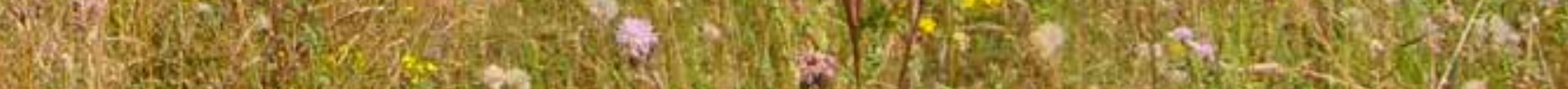

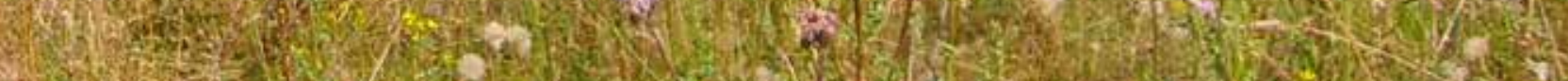

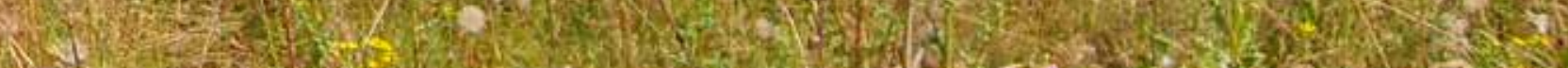

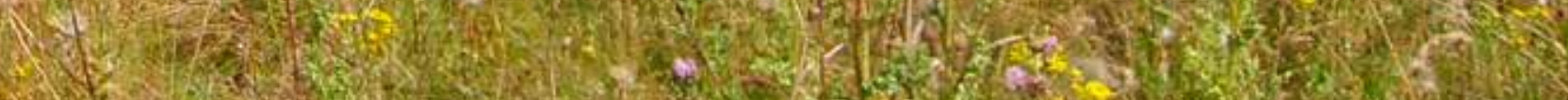
N

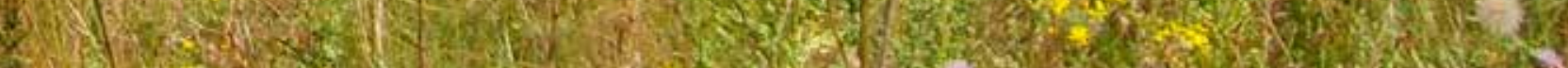


CHAPTER 7

Temporal carry-over effects in sequential plant-soil feedbacks

E. R. Jasper Wubs and T. Martijn Bezemer

Submitted manuscript 


\section{Abstract}

Plant-soil feedbacks (PSF) strongly influence plant performance. However, to what extent these PSF effects are persistent in the soil and how they are altered by species that subsequently condition the soil is unclear. Here we test how conspecific and heterospecific soil-conditioning effects interact across different soil-conditioning phases. We conducted a glasshouse experiment where six plant species conditioned soils, fully factorial, in two consecutive periods and measured the performance of Jacobaea vulgaris. The species that conditioned the soil during the second conditioning phase strongly determined the performance of J. vulgaris, but also the order and combination of species that conditioned the soil in the two phases accounted for a large part of the variance. For shoot biomass this interaction was the dominant variance component. We show that soil conditioning legacies carry-over and interact with the conditioning effects of succeeding plants. Between studies PSF effects on the same species can vary greatly even when abiotic conditions are the same. Our results suggest that this may be due to legacies of species that grew previously in the soil.

\section{Introduction}

Plant-soil feedback (PSF) can be an important driver of plant population dynamics (Van der Putten et al. 2013) and arises when a plant alters its abiotic and biotic soil environment which in turn affects the performance of a plant that subsequently grows in that location (Bever 1994, Ehrenfeld et al. 2005, Van der Putten et al. 2013). While plant-soil feedbacks are well studied, the temporal dynamics of PSF are poorly understood (Kardol et al. 2013, Hawkes et al. 2013). A common assumption is that there is a positive relationship between the time that a plant species conditions the soil and the PSF effect size (Kardol et al. 2013). However, in addition to conspecific soil conditioning, heterospecific PSF effects, where the soil is conditioned by another species, can also greatly influence plant performance (Bever et al. 1997, Bever 2003, Van de Voorde et al. 2011, Chapter 5). How sequential soil conditioning by different species affects PSF is unclear.

We postulate four hypotheses that predict how sequential soil conditioning influences PSF (Fig. 7.1a). First, the effects of sequential conditioning on PSF may be additive (Fig. 7.1a-i). In this way the performance of a plant growing in soil that is sequentially conditioned by two species that cause e.g. a negative PSF will be more reduced than when grown in soil exposed to a single conditioning period. This could be due to build-up of pathogen loads or accumulation of toxins in the soil (Packer and Clay 2000, Mazzoleni et al. 2015). Several studies have, indeed, 
a)

Conceptual models

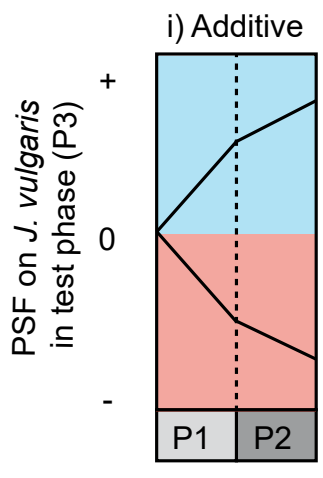

ii) Most recent

iii) Prior
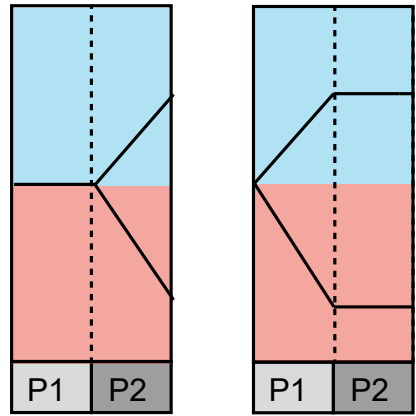

iv) Modification

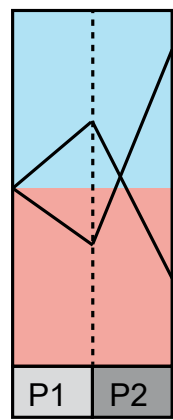

time -->

b)

Phase $1=$
Prior
conditioning

Phase 2 =

Most recent conditioning

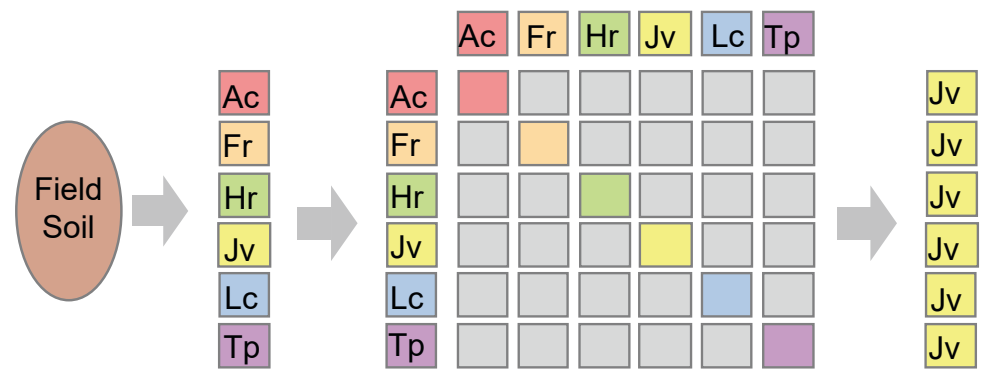

Fig. 7.1. Conceptual models of sequential PSF (a) and the experimental design used to test them (b).

a) Conceptual models of the temporal control on PSF. The first model postulates that PSF effects are additive over time, so that e.g. two species that generate negative PSFs will lead to more strongly negative PSF when they sequentially condition the soil than when either of them conditioned the soil only in one phase. The next two models posit full control of the feedback in the test phase by either ii) the most recent conditioning species (Phase 2; P2) or iii) the species that first conditioned the soil (Phase 1; P1). The last model (iv) allows for interactive effects among the two conditioning phases. b) Common field soil was conditioned by six species in a full-factorial design in two conditioning phases. In test phase (phase 3) Jacobaea vulgaris was grown as the test species in all pots, and the data of phase 3 are presented in this paper. Data from phase 1 and 2 can be found in Chapter 5. Abbreviations as in Fig 7.3.

shown that repeated soil conditioning by the same species can lead to increasingly negative conspecific PSF (Mazzola 1999, Packer and Clay 2004). Secondly, the plant that most recently conditioned the soil may exert full control over the PSF that is generated. This is plausible because soil communities are highly dynamic 
and their composition may turn-over substantially within weeks (Schadt et al. 2003, Bardgett et al. 2005). Hence, the identity of the plant that grew previously in the soil may not influence the current composition of the soil community and the resulting PSF effect (Fig. 7.1a-ii).

Thirdly, it is also plausible that PSF effects may be determined predominantly by the plant that previously conditioned the soil and not by the succeeding plant (Fig. 7.1a-iii). Plants may create long lasting biotic or abiotic legacies in the soil and via this mechanism they can influence plants that grow there later (Kulmatiski et al. 2006). Indirect support for this hypothesis comes from field experiments where changes in the composition of plant communities are often not related to changes in the soil community (Hedlund et al. 2003, Kardol et al. 2013) even though plants in those communities can greatly influence the composition of the soil community (Bezemer et al. 2010, Lundberg et al. 2012). Such legacy effects could be due to phytotoxins released by the plant that remain active in the soil and still influence plant growth later (Mazzoleni et al. 2015) or due to long-lasting legacy effects of plants on soil biota (Kostenko et al. 2012). For example, many soil biota form persistent resting stages that may remain in the soil for a long time awaiting the right conditions (e.g. presence of a host plant; Lennon and Jones 2011).

Finally, the current and preceding soil-conditioning species may have interactive effects on PSF (Fig. 7.1a-iv). The soil community is characterized by myriad interactions among its members (Cortois and De Deyn 2012) and plant species influence different groups of soil organisms in a highly specific manner (Grayston et al. 1998, Bezemer et al. 2010). Hence, it is plausible that these specific influences on different consortia of soil organisms by plants that succeed each other reshape the composition of soil communities, e.g. through competition and predation, in a manner that depends on the specific sequence of plants that have been growing in the soil. The PSF effect that results from these changes in the soil community will then depend on the identity of both the most recent and the preceding soilconditioning plants.

The aim of this paper was to elucidate whether sequential soil-conditioning by plants affects PSF responses of $J$. vulgaris as a proof of principle. We tested whether PSF is controlled mostly by prior plant-induced soil legacies, by the species that most recently conditioned the soil, or whether the PSF is determined by an interplay between the species that sequentially conditioned the soil (Fig. 7.1a). We conducted a glasshouse experiment where six plant species conditioned soil for 
two consecutive periods. All sequential combinations of the six species were tested (Fig. 7.1b). We subsequently assessed PSF in a test phase (Phase 3) by measuring the performance of one of the six species, Jacobaea vulgaris, on all soils. Jacobaea vulgaris is known to generate strong negative conspecific feedback and exhibits large variation in heterospecific soil feedbacks (Van de Voorde et al. 2011, Jing et al. 2015, Kos et al. 2015b, Chapter 5). We hypothesized that the second conditioning phase would influence PSF more strongly than the first conditioning phase (Fig. 7.1a-ii). However, as not all parts of the soil community are influenced equally by plants and plant species differ in which subsets of the soil community they influence (Grayston et al. 1998, Bezemer et al. 2010), we also expected important interactive effects among the two conditioning periods (Fig. 7.1a-iv). Finally, plantsoil interactions may be mediated by both abiotic and biotic factors (Ehrenfeld et al. 2005, Van der Putten et al. 2013). To tease these drivers apart we conducted the whole experiment both under ambient and fertilized nutrient levels. We predicted that with fertilization plant-soil feedback effects become smaller in magnitude, because plants will be better able to defend themselves against antagonists and they are less dependent on soil mutualists under high nutrient conditions (Reynolds et al. 2003, De Deyn et al. 2004, Kos et al. 2015a). However, we interpreted a positive correlation of the PSFs in the ambient and fertilized treatments as indirect evidence for soil biota as drivers of the feedbacks.

\section{Materials and Methods}

\section{Plants and growing conditions}

We used six species typical of grasslands that all naturally occur within the area of soil collection. We selected two grasses (Agrostis capillaris L. and Festuca rubra L.), two forbs (Hypochaeris radicata L. and J. vulgaris Gaertn.), and two legumes (Lotus corniculatus L. and Trifolium pratense L.). Seeds for each of the species were obtained from a specialized company that provides seeds from wild plants (Cruydt-Hoeck, Assen, the Netherlands) or collected from the same field as the soil (J. vulgaris). All seeds were surface-sterilized ( $1 \mathrm{~min}$. in $<2.5 \% \mathrm{NaClO}$ solution), rinsed with water and allowed to germinate on sterilized glass beads in a climate chamber (16:8-h day-night cycle, continuous $\left.20^{\circ} \mathrm{C}\right)$. The experiment was carried out in a glasshouse set to $16: 8 \mathrm{~h}$ day:night, $21: 16^{\circ} \mathrm{C}, \geq 60 \%$ relative humidity. Natural light was supplemented with $600 \mathrm{~W}$ metal-halide lamps, 1 per $4 \mathrm{~m}^{-2}$ (approx. $225 \mu \mathrm{mol}$ light quanta $\mathrm{m}^{-2} \mathrm{~s}^{-1}$ at plant level).

We collected topsoil (to $30 \mathrm{~cm}$ deep) from an ex-arable grassland (Mossel, Planken Wambuis, Ede, the Netherlands, GPS: $52^{\circ} 040 \mathrm{~N} 05^{\circ} 450 \mathrm{E}$ ) that had been under 
restoration for 17 years. Bags containing the collected soil were a priori allocated to one of three replicates and these soil replicates were kept separate throughout the experiment. The soil was sieved ( $5 \mathrm{~mm}$ mesh size) and used to fill containers (17 x $17 \times 17 \mathrm{~cm} ; 5 \mathrm{~kg}$ of soil per container). We subsequently conditioned the soil for eight weeks, by growing all six plant species in monocultures in the soil in a glasshouse (Phase 1). Sixteen individuals of a species were planted in each container and there were 58 or 77 replicate containers per species. Containers were placed randomly in the glasshouse. After eight weeks shoot biomass was clipped and major root systems were removed from the soil of each pot. Soil from containers in which the same species had grown and that were a priori allocated to the same soil-replicate, were pooled and homogenized. The soil was used for a large spatial plant-soil feedback experiment with homogeneous and heterogeneous soils (Chapter 5) and to obtain a sufficient amount of soil for the experiment, each of the 18 soil replicates ( 6 conditioned soils $\mathrm{x} 3$ replicates) was mixed with sterilized ( $>25 \mathrm{KGray}$ gamma radiation, Isotron, Ede, the Netherlands) field soil collected from the same site in a 8.4:1.6 (conditioned: sterile w:w) ratio. Containers (26 x 22 x $22 \mathrm{~cm} ; 1 \mathrm{x} \mathrm{w} \mathrm{x} \mathrm{h}$ ) were filled with $2.5 \mathrm{~kg}$ sterilized gravel (quartz, 4-8 mm) and then with $8 \mathrm{~kg}$ of conditioned soil and planted with 32 seedlings of a single species (Phase 2). All 18 conditioned soils were reconditioned for eight weeks with the same six species in a full factorial design ( 6 conditioned soils $\mathrm{x} 6$ plant species $\mathrm{x} 3$ replicates $=108$ containers). After eight weeks shoot biomass was clipped and soil was used for the test phase (Phase 3). For the current experiment only soil from containers with homogeneous soil were used. See Chapter 5 for further details.

\section{Test phase}

For the third phase all soils, that now had been conditioned for two phases, were sieved ( $5 \mathrm{~mm}$ mesh size) to remove roots and homogenized. Subsequently, the soil from each container of Phase 2 was divided over two new test pots $(10 \times 10 \times 11$ $\mathrm{cm} ; \mathrm{l} \times \mathrm{w} \times \mathrm{h}$ ), with $900 \mathrm{~g}$ soil in each pot. In addition, we created a treatment with unconditioned soil (UNC) taken newly from the same field as a control $(\mathrm{n}=12)$. We randomly selected one pot from each pair that came from the same container in Phase 2 for a fertilization treatment. We chose not to use soil sterilization as this also strongly alters soil nutrient availability (Troelstra et al. 2001, De Deyn et al. 2004) and leaves the soil prone to rapid microbial re-colonization as soon as it is exposed to a non-sterile environment such as a glasshouse. Pots were fertilized with $50 \mathrm{ml} 0.25$ strength Hoagland solution, while unfertilized pots received $50 \mathrm{ml}$ of demineralized water. Nutrients were added four times, once prior to planting and then in week 1, 3 and 5 of the experiment. Each pot was planted 
with three J. vulgaris seedlings and pots were placed in the glasshouse in a random spatial design. After a week we removed one seedling so that there were always two seedlings remaining per pot. Plants were allowed to grow for seven weeks and were watered regularly. Subsequently, shoots were clipped at the soil surface and the roots were removed from the soil of each pot. Shoot and root biomass were dried at $70{ }^{\circ} \mathrm{C}$ for $48 \mathrm{~h}$ and dry weights were determined.

\section{Data analysis}

Shoot and root data were analysed separately using linear mixed models with the Phase 1 soil replicate and the identity of the container in Phase 2 as nested random effects. Nutrient addition, and conditioning species identity in Phase 1 and Phase 2 as well as their interactions were included as fixed factors. For ease of interpretation the LMM analyses were also carried out for fertilized and unfertilized pots separately. We assessed the relative importance of soil conditioning effects from Phase 1 and Phase 2 and their interaction by calculating the partial $\mathrm{R}^{2}$ for each factor and interaction in the model (Grömping 2006). We used planned contrasts to analyse the differences in plant performance among the species that conditioned the soil in Phase 1 separately for each species that conditioned the soil during Phase 2 within the overall model (Adbi and Williams 2010, Brinkman et al. 2010). With the same approach we tested for differences in magnitude and sign of PSFs in both conditioning phases. The unconditioned soil treatment was not included in these models, because then the model could not be fully estimated (i.e. UNC was not crossed with the conditioning treatments in P1 and P2). Instead, we ran separate LMMs where all fixed effects, including the unconditioned soil treatment, were condensed into a single factor (i.e. analogous to a one-way ANOVA) and tested the overall effect of soil conditioning vs. unconditioned soils using planned contrasts. Finally, we correlated the PSF values obtained in control and fertilized pots, directly comparing each pair from the same Phase 2 container, to test whether soils had consistent effects on plant performance in fertilized and unfertilized pots. We calculated PSF as the log-ratio of the J. vulgaris biomass on the focal soil divided by the geometric mean of the biomass of J. vulgaris on all the soils, which is symmetric around zero (Brinkman et al. 2010).

All analyses were conducted in R v.3.3.0 ( $\mathrm{R}$ Core Team 2016) and model assumptions were checked graphically (Zuur et al. 2010). Heteroscedasticity was modelled using generalized least squares (Pinheiro and Bates 2000, Zuur et al. 2009). Linear mixed models were fitted with the NLME v.3.1-128 (Pinheiro et al. 2016) and the partial $\mathrm{R}^{2}$ values were extracted using RELAIMPO v.2.2-2 
(Grömping 2006). Planned contrasts were analysed within the MULTCOMP v.1.4-5 package (Hothorn et al. 2008).

\section{Results}

Fertilization strongly affected shoot and root biomass, and interacted with the effects of Phase 1 and Phase 2 conditioning (Table S7.1a). However, when analysed separately the effects of the two conditioning phases were qualitatively the same among fertilized and control pots (Table S7.2). For ease of interpretation we present the results of the unfertilized treatments first. However, the results of the fertilized half of the experiment are reported in full below (final paragraph of this section).

Soil conditioning in general led to lower plant biomass than in the unconditioned soil treatment (Table S7.1b). However, the identity of the species that conditioned the soil either during Phase 2 (the most recent period) or during Phase 1 had a clear impact on the PSF on J. vulgaris (Fig. 7.2, 7.3, Table S7.2a). Both shoot and root biomass of $J$. vulgaris during the third phase was affected more strongly by which species conditioned the soil during Phase 2 than by the identity of the species that conditioned the soil in Phase 1 (Fig. 7.2, Table S7.2a). However, the

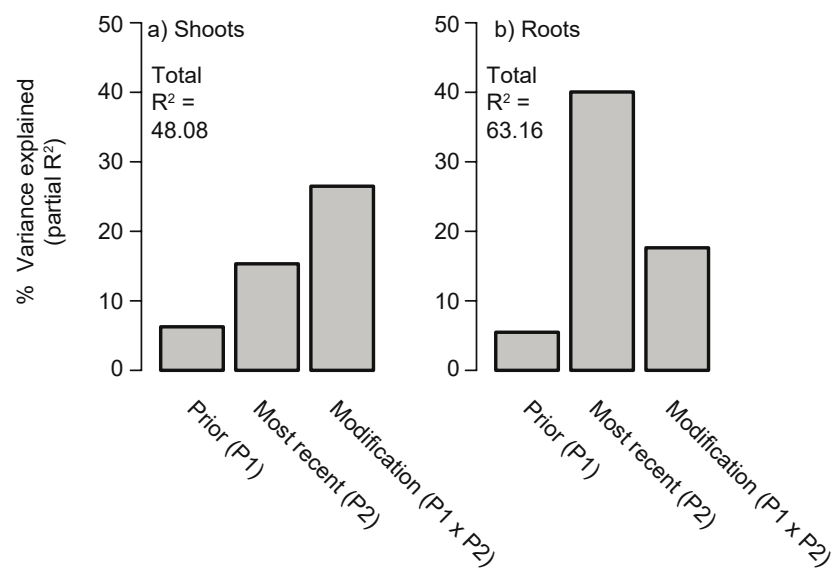

Fig. 7.2. Relative importance (partial $R^{2}$ ) of soil conditioning effects on J. vulgaris.

Shown are effects of prior conditioning (P1) phase and the most recent conditioning (P2) phase and their interaction on shoot (a) and root biomass (b) in soil conditioned by 3 6 combinations of six plant species without fertilization. 


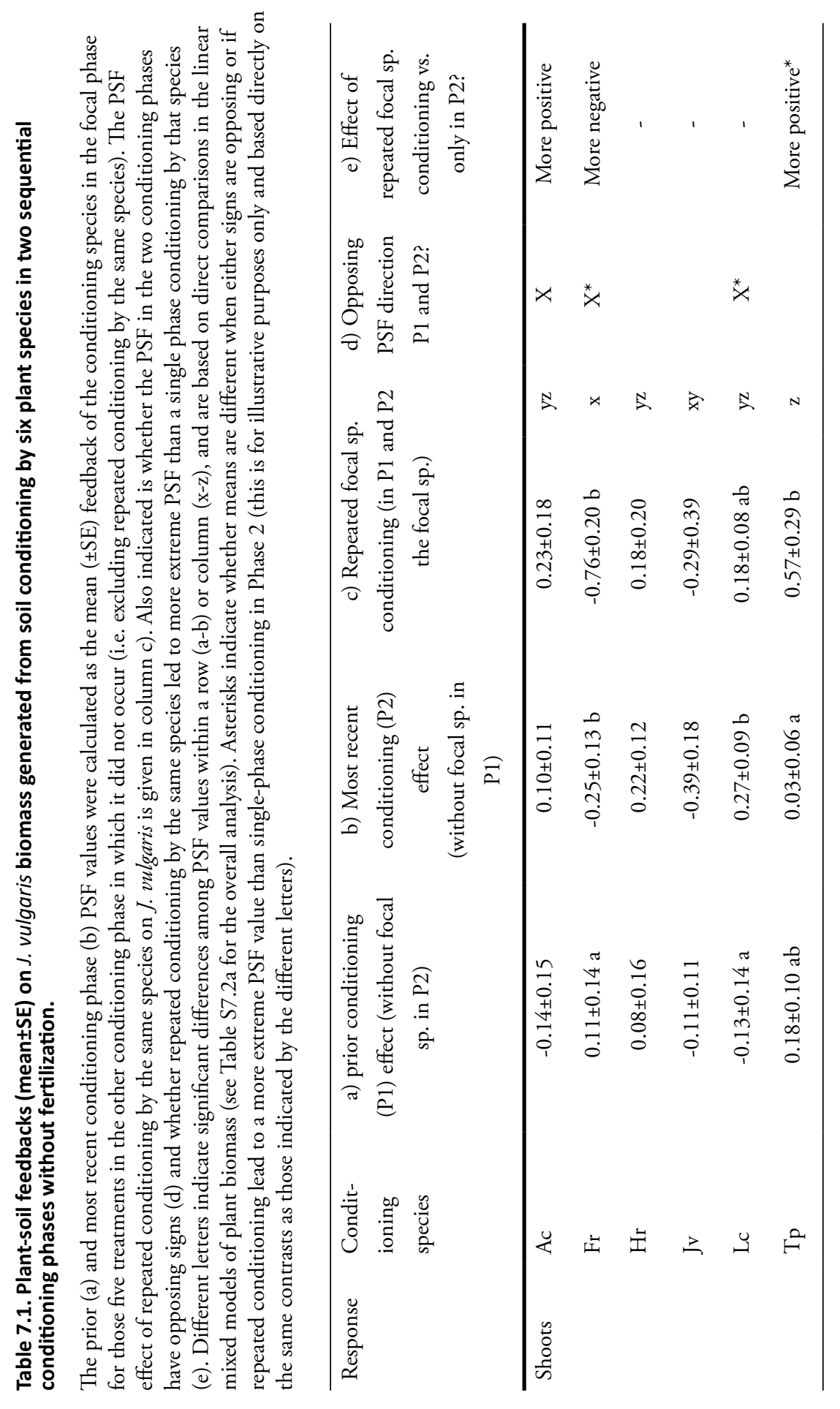




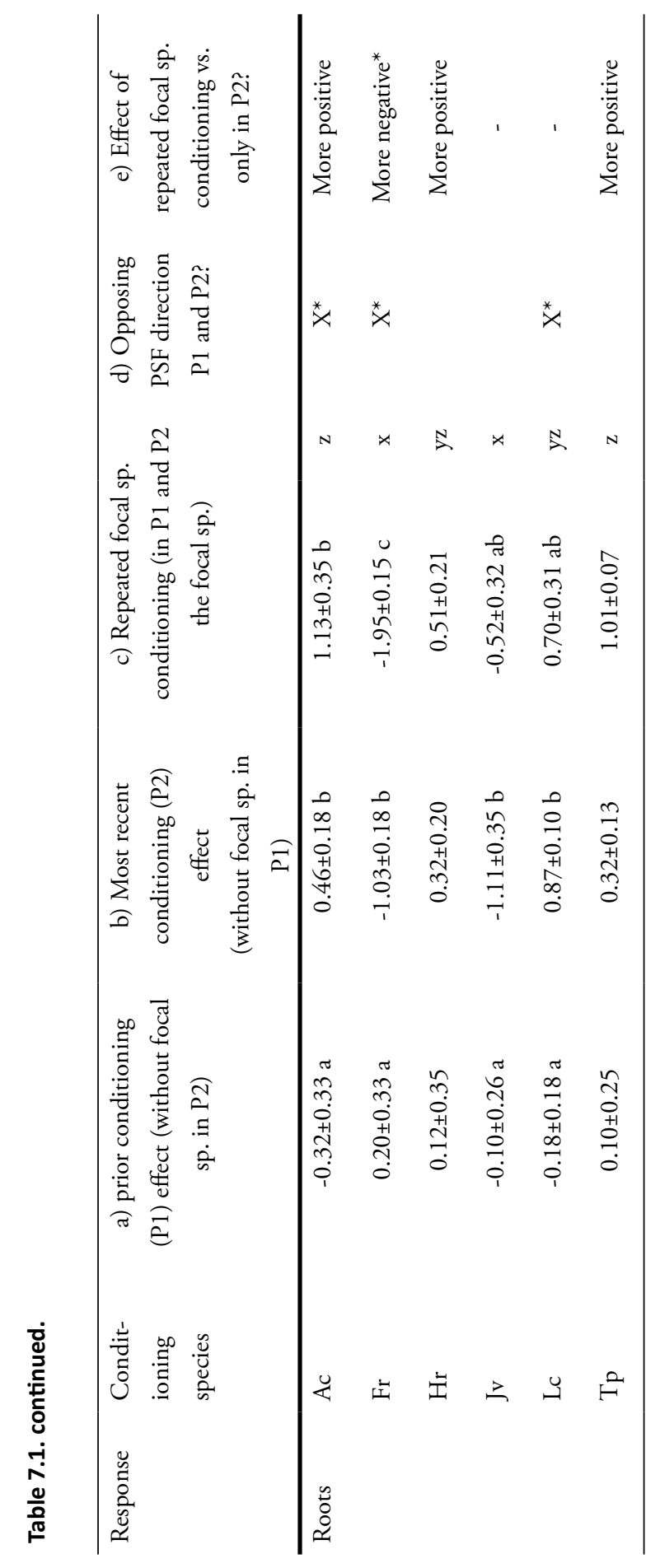


interaction among the two conditioning phases was also an important explanatory factor, and for shoot biomass it explained most of the variation (Fig. 7.2, Table S7.2a). This interaction indicates that PSF effect of a soil-conditioning species on $J$. vulgaris depends on the identity of the species that grew in that soil before.

The impact of the conditioning species on performance of $J$. vulgaris varied between the two conditioning phases. For three of the six species we found that the effect of the first conditioning phase was opposite in sign from the effect of the second conditioning phase (Table 7.1). For four out of six species the feedback effect on root biomass was more extreme, i.e. more positive or negative when the focal species was present in both conditioning phases compared to when the focal species was present only in Phase 2 (Table 7.1). For shoot biomass this was true for three species. However, this effect was only significant for $T$. pratense effects on shoot biomass and F. rubra effects on root biomass.

Soil conditioning by J. vulgaris in either the first or the second conditioning phase resulted in negative conspecific PSF (Table 7.1; Table S7.1). For root biomass, the effect generated in Phase 2 was more strongly negative than the effect generated in the first phase (Table 7.1). However, for this species, two successive phases of conspecific soil conditioning did not lead to stronger negative conspecific feedback, as this effect was intermediate to and not significantly different from the effect of the two conditioning phases in isolation. Two successive cycles of conditioning by F. rubra lead to PSF that was as negative for J. vulgaris as was the case for repeated conspecific soil conditioning (Figure 7.3a,b, blue arrows; Table 7.1).

Overall, the two plant species belonging to same plant family did not create consistent PSF effects on J. vulgaris root or shoot biomass and for most plant-pairs PSF effects differed significantly within the pairs (Fig. 7.3a-d). Only conditioning effects of $L$. corniculatus and $T$. pratense, both legumes (Fabaceae), during Phase 2 on $J$. vulgaris shoot biomass did not differ significantly.

As in the unfertilized treatment, in the fertilized pots we found that both the most recent conditioning phase (P2) and the interaction between the two conditioning phases were the dominant controls on the sequential PSF (Fig. S7.1, S7.2, Table S7.1, S7.2b). Also with nutrient addition did J. vulgaris experience negative conspecific PSF from both conditioning phases (Fig. S7.2c-f; Table S7.1), but two successive phases of conditioning by conspecifics did not lead to stronger negative 


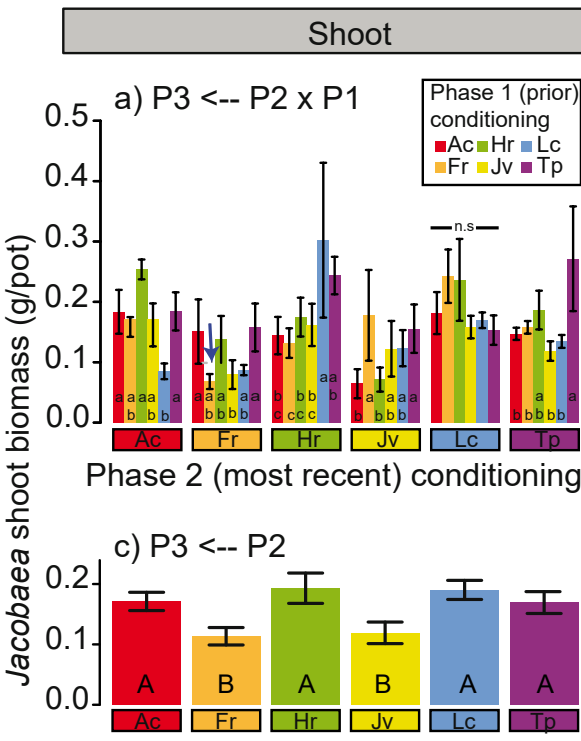

Phase 2 (most recent) conditioning
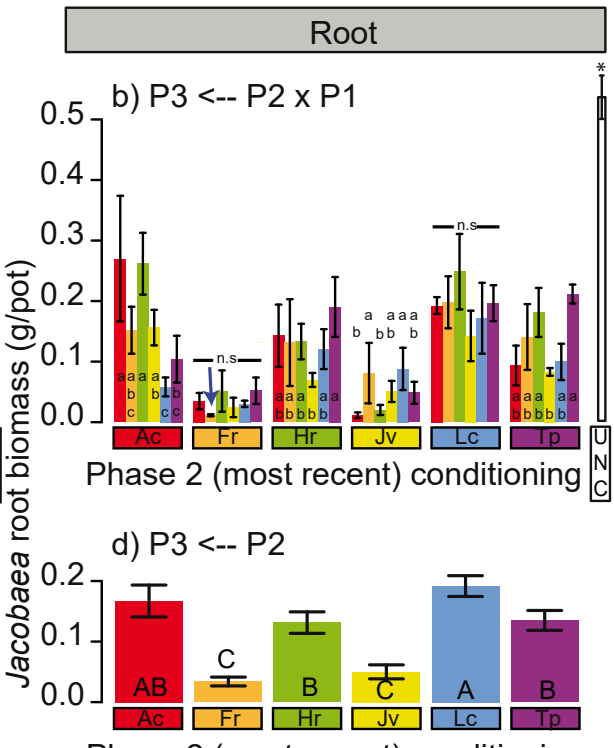

Phase 2 (most recent) conditioning

f) $P 3<--P 1$

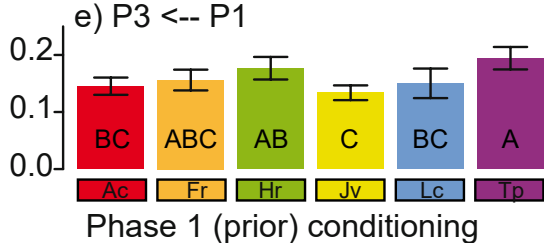

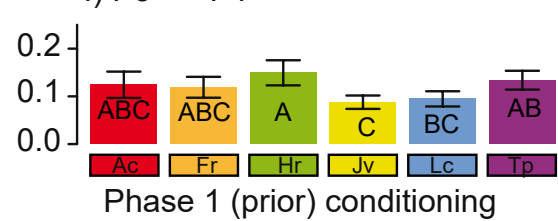

Fig. 7.3. Mean $( \pm S E)$ shoot $(a, c, e)$ and root $(b, d, f)$ biomass of $J$. vulgaris in the test $(P 3)$ phase in response to soil conditioned by 36 combinations of six plant species without fertilization.

The data for all 36 combinations of soil conditioning are shown in the top panels $(a, b)$. The biomass values obtained in the unconditioned control (UNC) treatment are indicated by the white bars in the top panels. An asterisk indicates that the conditioned soils were significantly different from the unconditioned control treatment. For ease of interpretation, the bottom two rows display the mean biomass for all pots conditioned by the same species in Phase 2 (c, d) or in Phase 1 (e, f) respectively. Significant differences among treatment means are indicated by different letters (see Table S7.2a for the overall analysis). To reduce the number of comparisons in the top row panels $(\mathrm{a}, \mathrm{b})$ differences among prior conditioning treatments were only tested within species that most recently conditioned the soil. Arrows indicate cases that are discussed in the main text. n.s. = not significant, $\mathrm{Ac}=$ Agrotis capillaris, $\mathrm{Fr}=$ Festuca rubra, $\mathrm{Hr}=$ Hypochaeris radicata, $\mathrm{Jv}=$ Jacobaea vulgaris, $\mathrm{Lc}=$ Lotus corniculatus, $\mathrm{Tp}=$ Trifolium pratense.

PSF (Fig. S7.2a,b). Both for shoots and roots, PSF values resulting from the different sequences of soil conditioning were positively correlated in the ambient 
and nutrient addition sub-experiments (shoot: Spearman's-rho $=0.18, \mathrm{n}=108, \mathrm{p}$ $=0.03$, root: rho $=0.34, \mathrm{n}=108, \mathrm{p}<0.001)$. However, the range of PSF values was smaller both above- and belowground when nutrients were added (Shoots: ambient: -1.6 to 1.4 vs. nutrients added: -0.6 to 0.7 , roots: ambient: -3.6 to 1.8 vs. nutrients added: -1.5 to 0.8 ; paired t-test for differences in absolute PSF values: shoots: $\mathrm{t}_{107}=-6.4, \mathrm{p}<0.0001$, roots: $\mathrm{t}_{107}=-7.1, \mathrm{p}<0.0001$ ).

\section{Discussion}

We show that the particular sequence of species that grew in the soil before determines the resulting PSF. While the feedback effect of the most recent conditioning phase was an important factor, the interplay of conditioning effects among the two phases also played a key role. Soil conditioning in the first phase generates PSF, which is then modified by the plant species that subsequently conditions that soil to ultimately determine the feedback effects in third phase. The non-additive nature of the interaction among soil conditioning phases is clearly illustrated by the switch in the sign of the generated feedback, from positive to negative or vice versa, in the two conditioning phases for half of the tested species. In addition, we found that repeated conditioning by the same species could lead to feedbacks that were more extreme than was expected based on the feedback effects when that species conditioned the soil only once in phase 2 .

Most plant-soil feedback experiments to date use only one period of soil conditioning and these data have been the basis for models of PSF effects on plant community changes (Bonanomi et al. 2005, Eppinga et al. 2006, Fukami and Nakajima 2013, Mack and Bever 2014, Abbott et al. 2015). As far as we are aware, only two experiments tested the effects of repeated conditioning by conspecifics (Mazzola 1999, Packer and Clay 2004), and we are not aware of any sequential heterospecific feedback experiments. In contrast to these two published sequential feedback studies, we did not find that repeated conspecific conditioning lead to more negative PSF. In addition, we found that particular combinations of heterospecific conditioning could lead to strong negative feedback, comparable to two phases of conspecific conditioning (e.g. repeated conditioning by F. rubra). This suggests that sequential heterospecific feedbacks are also important in determining the PSF and consequently plant performance. Work from our own group on heterospecific PSF effects on J. vulgaris shows that species-specific soil conditioning effects are not always consistent across studies (c.f. Van de Voorde et al. 2011, Jing et al. 2015, Kos et al. 2015b) even though the soils used in these studies originate from the same site. Our results suggest that prior soil conditioning (i.e. 
the plant species that conditioned the soil in the field when it was collected) may create soil legacies that subsequently influenced the species-specific conditioning effects in the glasshouse experiments. However, the extent to which this plays a role in natural conditions needs to be explored.

This study was intended as a proof of principle, made possible by the availability of sequentially conditioned soils from another experiment (Chapter 5). One of the limitations of this study is that in the first phase 16 individuals were used to condition soil, while in the second phase 32 plants were used. Although the amount of soil conditioned per individual was not very different among the two phases ( 0.31 vs. $0.25 \mathrm{~kg}$ per individual in Phase 1 and 2 respectively), it is perhaps not surprising that we find stronger soil conditioning effects of Phase 2 since here the density of conditioning plants was higher. However, our major point here is that PSF effects can carry-over from one conditioning phase to the next, even despite the lower number of conditioning plants in Phase 1. For logistic reasons we were restricted to using a single test species. However, if these results apply to more species, then models of plant community dynamics based on a single conditioning phase do not capture the dynamics of plant-soil interactions sufficiently. For instance, our data suggests that the common modelling assumption that a period of heterospecific conditioning removes the negative feedback of prior conspecific conditioning may be wrong (Bonanomi et al. 2005, Eppinga et al. 2006). This may also apply to models of crop-rotation performance when only a single conditioning phase, i.e. only the preceding crop, is taken into account (Leoni et al. 2013, Dias et al. 2015).

Mechanistic understanding and prediction of plant-soil feedbacks is still in its infancy (Cortois and De Deyn 2012, Van der Putten et al. 2016) and PSFs can be mediated by both abiotic and biotic drivers (Ehrenfeld et al. 2005). In our experiment, we also found a complex interplay between soil conditioning in the two phases when we conducted the same experiment with nutrient addition. Furthermore, the observed PSF responses in the ambient and added nutrients sub-experiments were positively correlated. Therefore, we suggest that in our study the observed feedbacks result primarily from biotic interactions in the soil, although we cannot fully exclude a role of abiotic factors and allelochemicals (e.g. Mazzoleni et al. 2015). Nutrient addition did cause the observed PSFs to be smaller in magnitude, which is in line with the idea that in high nutrient environments plants are better able to defend themselves against soil pathogens and are less dependent on soil-borne mutualists (Reynolds et al. 2003, De Deyn et al. 2004, 
Kos et al. 2015a). In general, soil conditioning led to a lower performance of $J$. vulgaris, which is in line with earlier work showing that most plant species exert a negative heterospecific PSF on J. vulgaris (Van de Voorde et al. 2011).

In our experiment we used two species each of the Poaceae, Asteraceae and Fabaceae families. However, in line with other studies, the PSF generated by these species pairs was very different, confirming that phylogeny is a poor predictor of plantsoil feedbacks (Mehrabi and Tuck 2015, Mehrabi et al. 2015, but see Anacker et al. 2014). Other studies have suggested that plant functional traits, e.g. those associated with intrinsic growth rates, can be used to predict plant-soil feedback (Baxendale et al. 2014, Bardgett et al. 2014, Lemmermeyer et al. 2015) if they can be related to the mechanism driving the soil feedback (Kardol et al. 2013). A way forward might be to study how different species affect the abundance of antagonists and mutualists during soil conditioning as an effect trait (sensu Lavorel and Garnier 2002), as well as the sensitivity of the plant species to these organism groups as a response trait in order to predict the soil feedback generated by different plant species sequences (Cortois 2015, Van der Putten et al. 2016).

The temporal dynamics of PSF are highly complex as the experimentally observed feedbacks can depend on, for instance, the length of the feedback phase (Hawkes et al. 2013) and the life stage of the test plants (Kardol et al. 2013). Our study species, J. vulgaris, is biennial, but often grows vegetatively for multiple years (Van der Meijden and Van der Waals-Kooi 1979). Thus whether insights on PSF generated by relatively short periods of soil conditioning (eight-weeks in our study) translate faithfully to performance in the field is unclear (Kulmatiski and Kardol 2008, Cortois and De Deyn 2012), although a recent study suggests that this is possible (Kulmatiski et al. 2016). However, in the field PSFs will be further modified by factors such as interspecific plant competition, nutrient availability and herbivory (Veen et al. 2014, Van der Putten et al. 2016) and will not be affected by procedural artefacts such as soil sieving and homogenization, making predictions harder still. Nevertheless, we think our experiment demonstrates the potential role that modification of PSFs by prior soil conditioning (i.e. sequential conditioning) can play in determining plant performance. Future studies should combine long-term observations of plant species replacements in the field with targeted experiments where the consequences of soil conditioning on soil community composition and plant responses are explicitly studied to understand the role of sequential PSFs in the field. 
In conclusion, we show that the sequence of species that condition the soil can impact the sign and magnitude of plant-soil feedback, both with and without fertilization. Prior soil conditioning importantly modifies the PSF effects generated by the species that next conditions the soil. We also show that heterospecific conditioning could result in equally negative PSF as repeated conspecific soil conditioning. Our results highlight the need to incorporate sequential heterospecific feedbacks in models of plant community dynamics as well as in the design of effective croprotation schemes. 


\section{Supplementary information}

Table S7.1. Results from LMM analyses of soil conditioning effect in two subsequent phases with and without nutrient addition on J. vulgaris shoot and root biomass.

A) full model with all interactions among fixed effects, but excluding the unconditioned (UNC) soil treatment. Planned contrasts for the effect of conditioning by J. vulgaris in the two phases are shown. B) LMMs with all fixed factors analysed as a one-way analysis and including the unconditioned soils as a treatment. Planned contrasts are shown comparing plant biomass in UNC to the average biomass across all conditioned soil treatments.

\begin{tabular}{|c|c|c|c|c|c|c|c|}
\hline \multicolumn{2}{|c|}{ A) three-way model (excl. UNC) } & \multicolumn{3}{|c|}{ Shoots } & \multicolumn{3}{|c|}{ Roots } \\
\hline Terms/contrasts & d.f. & $\mathrm{F}$ & $\mathrm{Z}$ & p-value & $\mathrm{F}$ & $\mathrm{Z}$ & p-value \\
\hline Nutrients & 1,72 & 605.81 & & $<0.0001$ & 601.81 & & $<0.0001$ \\
\hline P2 soil & 5,60 & 9.08 & & $<0.0001$ & 32.37 & & $<0.0001$ \\
\hline $\mathrm{Jv}$ in $\mathrm{P} 2-$ cont & & & -4.18 & $<0.0001$ & & -7.24 & $<0.0001$ \\
\hline $\mathrm{Jv}$ in $\mathrm{P} 2-$ nutr & & & -2.83 & 0.0047 & & -3.87 & 0.0001 \\
\hline P1 soil & 5,12 & 1.47 & & 0.2710 & 2.44 & & 0.0955 \\
\hline $\mathrm{Jv}$ in $\mathrm{P} 1-\mathrm{cont}$ & & & -1.88 & 0.0601 & & -2.51 & 0.0121 \\
\hline $\mathrm{Jv}$ in $\mathrm{P} 1$ - nutr & & & -0.72 & 0.4719 & & -3.52 & 0.0004 \\
\hline $\mathrm{N}^{*} \mathrm{P} 2$ & 5,72 & 1.71 & & 0.1438 & 2.63 & & 0.0308 \\
\hline $\mathrm{N}^{*} \mathrm{P} 1$ & 5,72 & 1.21 & & 0.3141 & 3.45 & & 0.0075 \\
\hline $\mathrm{P} 2 * \mathrm{P} 1$ & 25,60 & 1.72 & & 0.0451 & 1.97 & & 0.0166 \\
\hline \multirow[t]{2}{*}{$\mathrm{N} * \mathrm{P} 2 * \mathrm{P} 1$} & 25,72 & 1.78 & & 0.0307 & 0.97 & & 0.5130 \\
\hline & & \multicolumn{3}{|c|}{ Shoots } & \multicolumn{3}{|c|}{ Roots } \\
\hline $\begin{array}{l}\text { B) one-way model } \\
\text { (incl. UNC) }\end{array}$ & d.f. & $\mathrm{F}$ & Z & p-value & $\mathrm{F}$ & $\mathrm{Z}$ & p-value \\
\hline Treatment & 73,41 & 12.02 & & $<0.0001$ & 14.35 & & $<0.0001$ \\
\hline \multicolumn{2}{|c|}{ UNC vs conditioning - cont } & & -5.59 & $<0.0001$ & & -6.12 & $<0.0001$ \\
\hline \multicolumn{2}{|c|}{ UNC vs conditioning - nutr } & & -6.03 & $<0.0001$ & & -5.08 & $<0.0001$ \\
\hline
\end{tabular}


Table S7.2. Results from GLM analyses of soil conditioning effect in two subsequent phases on J. vulgaris shoot and root biomass for sub-experiments with ambient (a) and added nutrients (b).

These analyses are shown for ease of interpretation: for a combined analysis of the two subexperiments see Table S7.1.

\begin{tabular}{|c|c|c|c|c|c|c|c|c|c|}
\hline \multirow[b]{3}{*}{ Terms } & \multirow[b]{3}{*}{ d.f. } & \multicolumn{4}{|c|}{ a) Unfertilized } & \multicolumn{4}{|c|}{ b) Fertilized } \\
\hline & & \multicolumn{2}{|c|}{ Shoot } & \multicolumn{2}{|c|}{ Root } & \multicolumn{2}{|c|}{ Shoot } & \multicolumn{2}{|c|}{ Root } \\
\hline & & $\mathrm{F}$ & $\mathrm{p}$-value & $\mathrm{F}$ & p-value & $\mathrm{F}$ & $\mathrm{p}$-value & $\mathrm{F}$ & $\mathrm{p}$-value \\
\hline $\mathrm{P} 2$ & 5,60 & 8.94 & $<0.0001$ & 27.94 & $<0.0001$ & 8.26 & $<0.0001$ & 7.60 & $<0.0001$ \\
\hline P1 & 5,12 & 2.04 & 0.1443 & 1.98 & 0.1548 & 1.31 & 0.3253 & 2.82 & 0.0658 \\
\hline $\mathrm{P} 2 \times \mathrm{P} 1$ & 25,60 & 1.84 & 0.0276 & 1.92 & 0.0206 & 1.81 & 0.0319 & 1.18 & 0.2980 \\
\hline
\end{tabular}

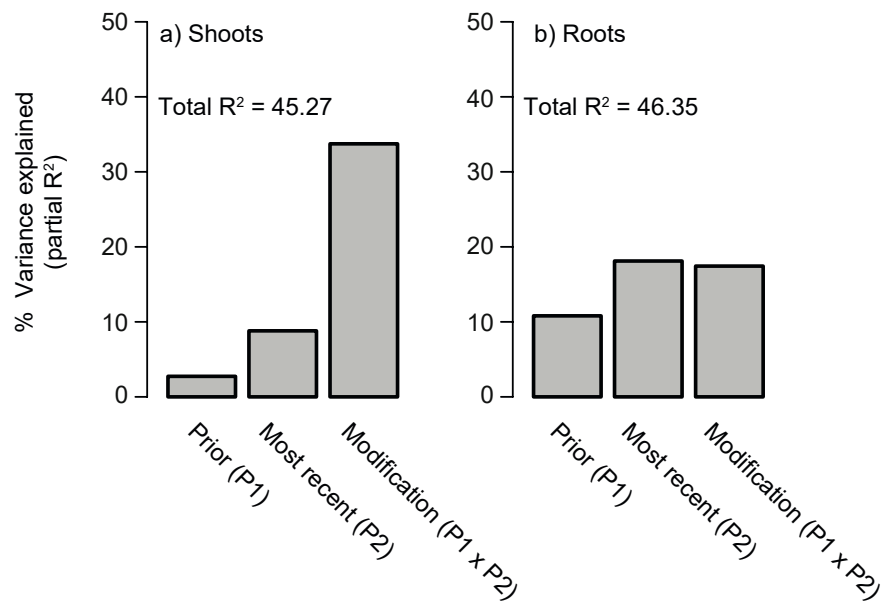

Fig. S7.1. Relative importance (partial $\mathrm{R}^{2}$ ) of soil conditioning effects on J. vulgaris.

Shown are effects of the prior conditioning (P1) phase and the most recent conditioning (P2) phase and their interaction in determining shoot (a) and root biomass (b) in soil conditioned by 36 combinations of six plant species in the sub-experiment with nutrient addition (c.f. Fig. 7.2). 

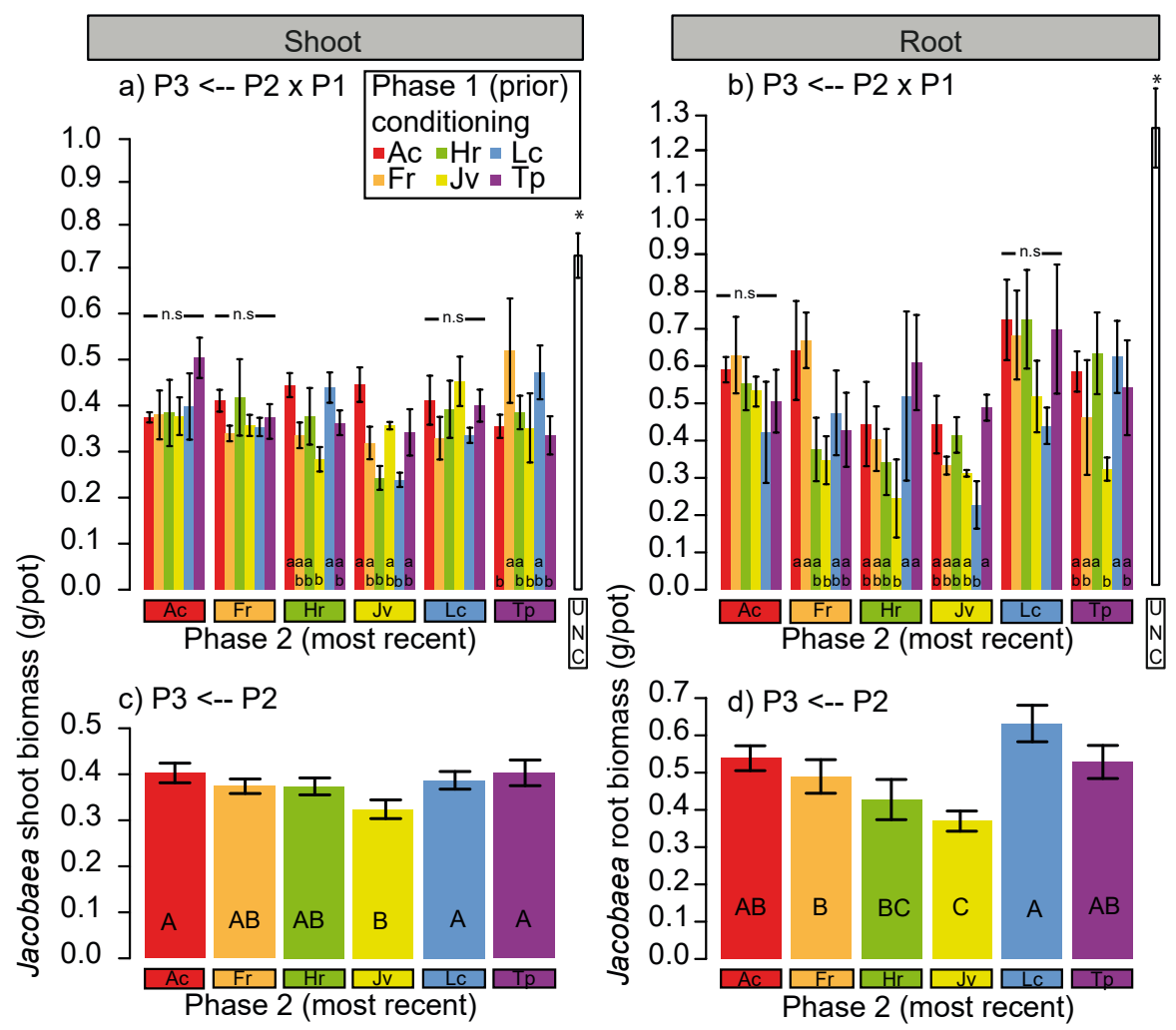

e) P3 <-- P1
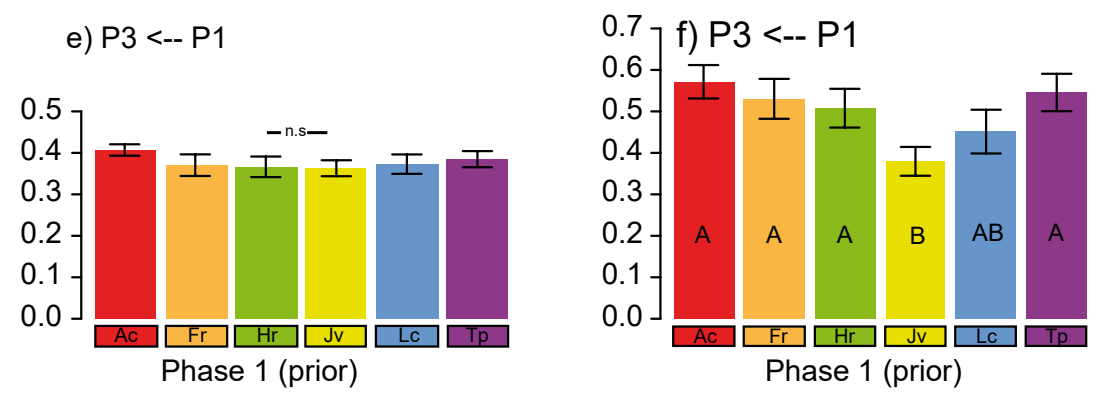

Fig. S7.2. Mean ( $\pm S E)$ shoot $(a, c, e)$ and root $(b, d, f)$ biomass of $J$. vulgaris in the test (P3) phase in response to soil conditioned by 36 combinations of six plant species in the subexperiment with nutrient addition (c.f. Fig. 7.3).

In addition, the biomass values obtained in the unconditioned control (UNC) treatment are indicated by the white bars in the top panels. Abbreviations and conventions as in Fig. 7.3. 


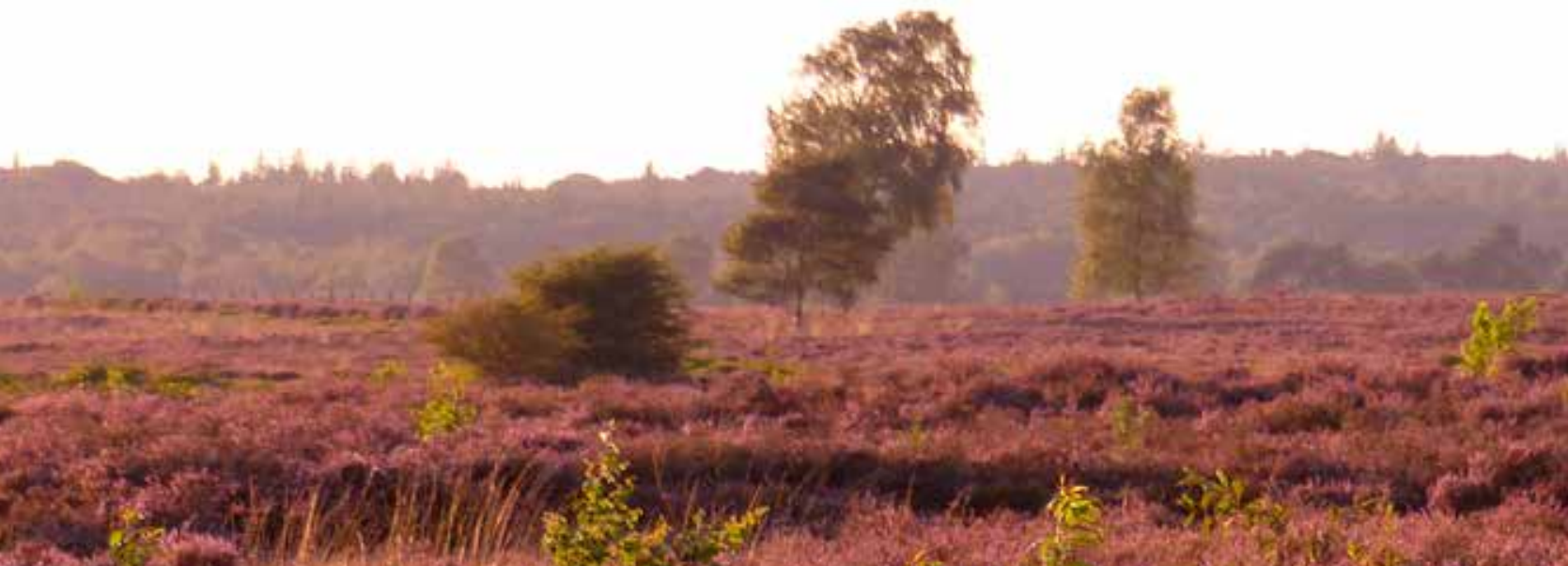

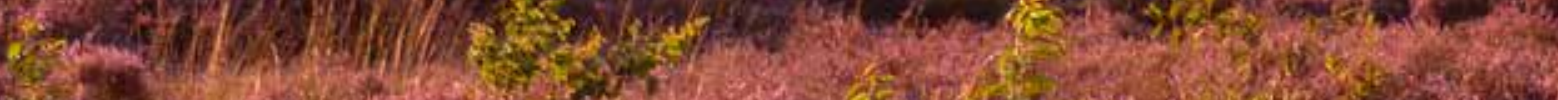
7.

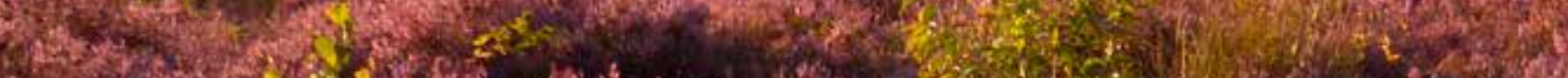
2.2.

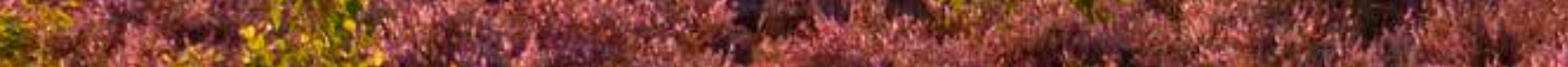

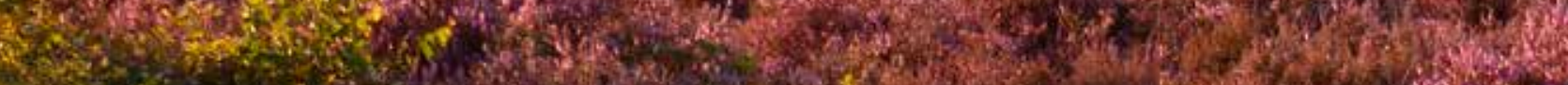
-

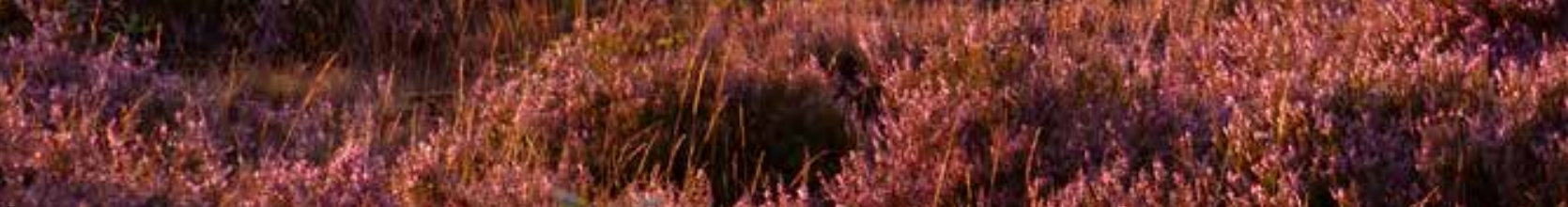

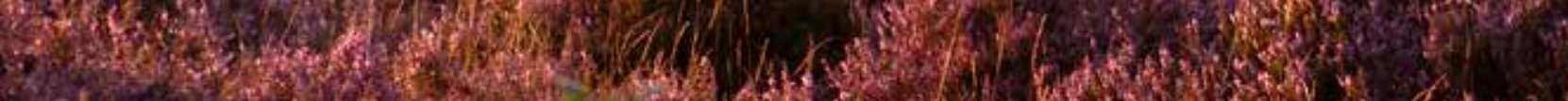

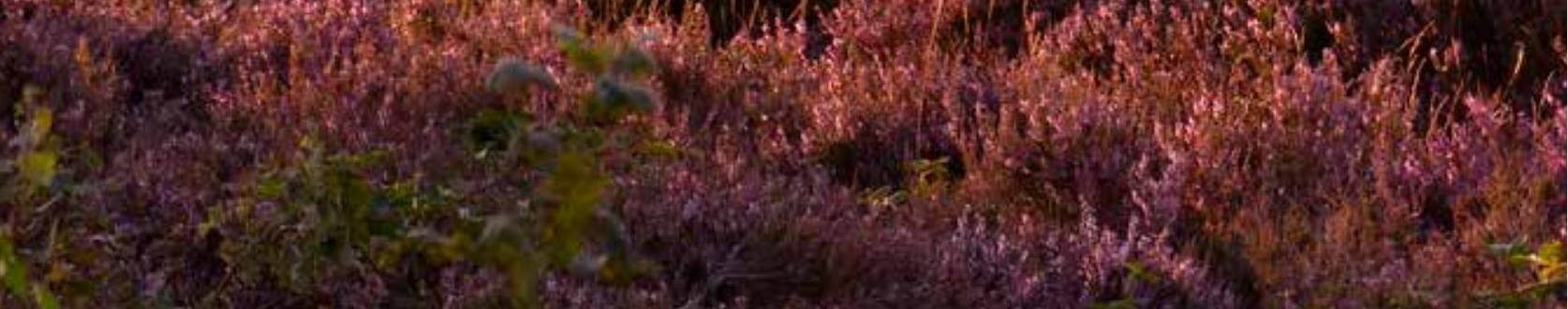


CHAPTER 8

General discussion and synthesis 
Community developments during succession, and by extension restoration (Walker et al. 2007), are the result of initial plant and soil community composition, local abiotic conditions, colonization by new species, and the complex web of plant and soil community interactions that ensue (Fig. 8.1). I have studied how plant-soil interactions influence natural community dynamics and how successional dynamics can be altered by intervening in these interactions and potentially improve nature restoration as a result. Here, I will place these results in a wider context.

\section{Plant-soil interactions can speed-up nature restoration}

In terms of biodiversity and conservation values some ecosystem states may be considered desirable, while others are undesirable. Human management, such as arable cultivation, alters the abiotic and biotic environment and this management may have left important legacies that will subsequently influence successional dynamics and restoration when these systems are to be restored to a target community (Mitchell et al. 2000, Kulmatiski et al. 2006, Cramer et al. 2008, Jackson and Hobbs 2009). In some cases human influence has been limited and natural community interactions will cause the system to shift to the desired

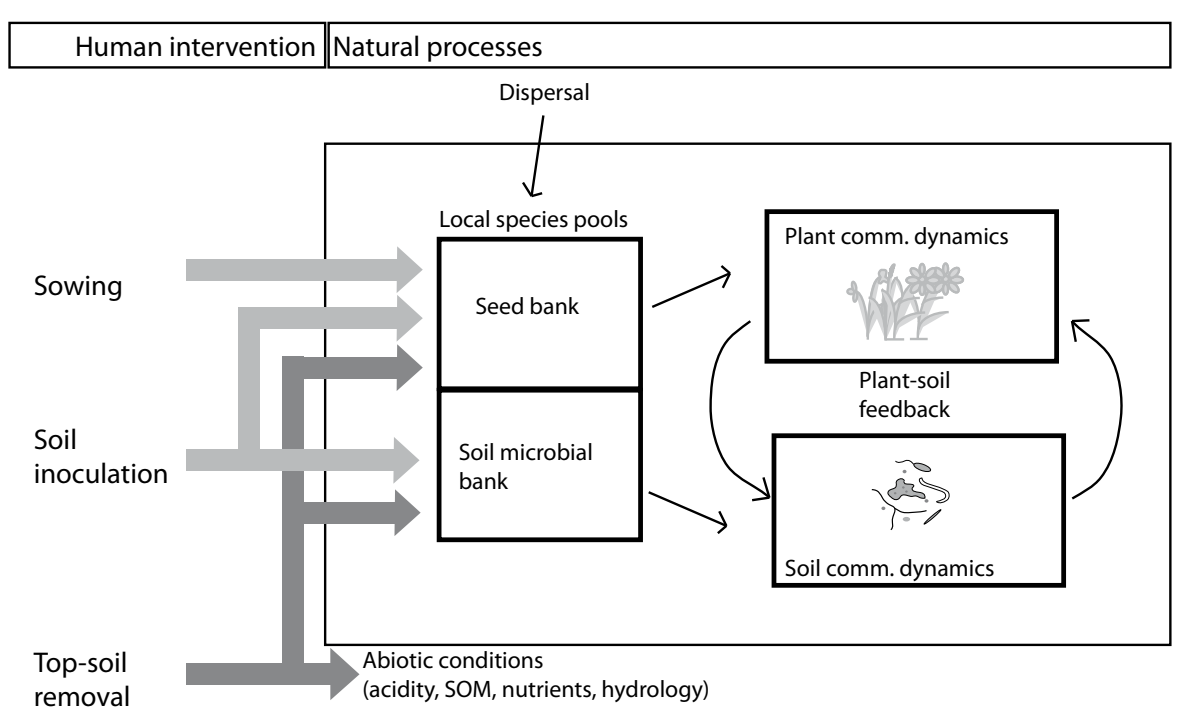

Fig. 8.1. Simplified conceptual framework of community development during secondary succession.

Thin black arrows indicate natural processes while thick grey arrows show human management practises in nature restoration. Only those processes and interventions that are discussed in this thesis are shown. 
ecosystem state unassisted (Cramer et al. 2008, Bartha et al. 2014, Prach et al. 2014). However, in many cases human actions will have caused the system to cross certain biotic or abiotic thresholds, which will prevent unassisted transition to a desired ecosystem state within a reasonable timeframe (Cramer et al. 2008, Isbell et al. 2013). A range of restoration measures or nature management practises can be implemented to re-cross these thresholds (e.g. Marrs 1993, Bakker and Berendse 1999, Prach et al. 2007). Only those that will be discussed here are highlighted in Fig. 8.1.

By evaluating a field experiment, the 'Reijerscamp' experiment, which was executed at a spatial scale that is relevant for restoration practise (c.f. Prach et al. 2007), I showed that soil inoculation can enhance the successional developments after topsoil removal (Chapter 2). This is in line with soil transfer studies also executed at a large spatial scale (Vécrin and Muller 2003, Pywell et al. 2011, Box et al. 2011, Bulot et al. 2014, Jaunatre et al. 2014). What is new, however, is that by using different soil inocula sources, the direction of succession can be steered towards different target communities. It is well known that depending on the species that colonize during old-field succession the community development may take very different trajectories in terms of species composition (Bartha et al. 2014, Fukami 2015). I have now demonstrated that it is possible to shift the direction of community developments to a different trajectory by using soil inocula of the respective target communities. Such an intervention may allow us to much more subtly manage successional developments (Marrs 2016).

Methods such as soil transfer or turf translocation have generally been applied in order to introduce appropriate seeds and vegetative plant propagules into restoration areas (Bullock 1998). Others have also highlighted the potential to cointroduce soil biota using these methods (Prach et al. 2007, Kardol et al. 2009). The introduction of seeds and propagules of target plant species is of course important, as many target species are relatively poor dispersers (Bakker and Berendse 1999, Prach et al. 2007). However, the co-introduction of soil biota with the plant material may also be key to successful restoration. It has been shown that late-successional soil communities can alter the competitive balance among ruderal and target plant species in favour of later successional plants (Kardol et al. 2006, Carbajo et al. 2011, Brinkman et al. 2012). In a parallel mesocosm experiment I could show that even when seed availability is kept constant, the two different soil inocula drive plant community composition in the direction of their respective donor communities (Chapter 2), thus mirroring the patterns observed 
in the field experiment. This indicates that along with the introduction of seeds, in the field soil biota also have helped to shape the successional trajectories. More importantly, however, this shows that the composition of the soil community may play a steering role in selecting which plant community will develop. This suggests that soil communities may be optimized, in terms of their composition, with respect to the restoration targets.

\section{Soil microbiome engineering for restoration}

The optimization of microbial communities, including those in soils, for specific functions is a very young and rapidly growing field called microbiome engineering (Mueller and Sachs 2015, Rillig et al. 2016a). So far this work has mainly been restricted to animal and plant model species and functions of interest to agricultural production. For instance, it could be shown that early- vs. late-flowering lines of Arabidopsis thaliana selected for soil microbiomes that, when inoculated into sterilized soil, could induce early and late flowering in other $A$. thaliana genotypes and in Brassica rapa (Panke-Buisse et al. 2015). These authors mixed soils sampled from agricultural, grassland and forest sites in order to create a source inoculum with maximum microbial diversity. When entire microbial communities that normally are spatially well segregated are mixed this is called a community coalescence event (Rillig et al. 2015, 2016a, b). Due to the large numbers of taxa occurring in the soil microbiome and the multitude of interactions among them, the outcome of this coalescence will result in a novel species composition, with potentially degraded or improved function (Mueller and Sachs 2015).

In Chapter 3 a community coalescence experiment with the explicit goal to optimize nature restoration is reported. Here, soil inocula from all pairwise combinations of arable field, mid-succession grassland and heathland, were tested in a search for synergistic effects on target and ruderal plant species performance. As was observed before (Chapter 2), heathland soil consistently improved target species growth in a dose-dependent manner. However, in addition, it was observed that a 50:50 mixture of arable and heathland soil led to a strong reduction in ruderal species growth. This reduction was stronger than expected based on simple dose-dependent (additive) responses. This illustrates that soil inocula can be engineered and that potential synergistic effects may be achieved through community coalescent methods (Rillig et al. 2015, 2016a, b). However, the effect size differed among the tested species, showing that depending on the desired species in the vegetation, different soil inoculum mixtures may be required. Nevertheless, across several experiments late-successional soil microbiomes consistently promoted growth of 
the late-successional target plant species (Kardol et al. 2006, Carbajo et al. 2011, Middleton and Bever 2012, Brinkman et al. 2012, Bauer et al. 2015), providing a solid starting point for testing microbiome engineering in nature restoration.

\section{Soil inoculation effects are context dependent}

Using data from a second field experiment, the 'Stepping Stone' experiment initiated by the EU-CLUE project in 1996, I was able to assess the long-term consequences of inoculating mid-successional soil as well as sowing mid-succession species for plant and nematode community composition (Chapter 4). The results show that sowing had a substantial impact on vegetation composition that lasted at least two decades. Soil inoculation primarily influenced nematode community composition. The effect was most pronounced within the first eight years, but was still detectable after 15 years of monitoring. This shows that soil inoculation can change the composition of the soil community for a long time.

However, in contrast the Reijerscamp experiment (Chapter 2), the effect of soil inoculation on the vegetation was relatively small in the Stepping Stone experiment (Chapter 4) and lasted only three years. The two experiments differ in several important respects, for instance one was large-scale and grazed by cattle (Reijerscamp; Chapter 2), while the other was relatively small-scale and annually mown and the hay removed (Stepping Stone; Chapter 4). Small scale inoculations may be less effective as the population sizes of inoculated organisms are smaller and thus have larger risks of extinction. However, the nematode community was substantially altered by soil inoculation, suggesting that the inoculation was effective in introducing soil organisms. Similarly, large herbivores may overrule plant-soil interactions (Schrama et al. 2013, Veen et al. 2014), but then I would expect the soil inoculations to be less effective in the grazed experiment and not the other way around. While I cannot rule out these hypotheses, I propose two other mechanism as potential explanations for this difference.

The Stepping Stone experiment was conducted on an intact arable topsoil directly and it has been shown that soil inoculation in an organic topsoil is less effective than following topsoil removal (Carbajo et al. 2011, Chapter 2). The fertile topsoil environment, with abundant microbial and invertebrate soil community as well as ample ruderal plant propagules, may present an important barrier to the establishment of both later-successional plants and soil biota. Higher nutrient conditions can importantly dampen plant-soil feedback effects (De Deyn et al. 2004, Manning et al. 2008, Kos et al. 2015, Chapter 7). Similarly, an abundant 
and diverse resident microbial community is more able to resist invasion (Van Elsas et al. 2012, Mallon et al. 2015, Calderón et al. 2017). Consequently, the presence of ruderal plant propagules and an arable soil community may have prevented a change in plant-soil interactions, which as a result could not facilitate the transition to a later-successional state.

Another key determining factor of the outcome of community coalescent processes is the species composition of the communities that are brought into contact (Rillig et al. 2016a). For instance, it was recently shown that soil microbial community restoration was either successful or not depending both on the resident community composition as well as the composition of the inoculated community (Calderón et al. 2017). In general, it is likely that taxonomically and functionally similar communities will coalesce without much alterations and the resulting species abundances mainly resulting from initial differences in abundance. Very dissimilar communities may lead to much more unpredictable outcomes in terms of soil community composition, and plant responses, after coalescence. As such it is striking that the heathland inoculum material led to more pronounced changes in community composition than the grassland-inoculum (Chapter $2 \& 3$ ). The donor heathland sites have been managed as heathland vegetation for centuries (Breman et al. 2009), while the grassland had been an actively cultivated arable field until three decades ago (Kardol et al. 2005, Van der Wal et al. 2006). Assuming that the grassland was still undergoing successional changes, while the heathland had reached a dynamic equilibrium state (plagioclimax), it is likely that the differences in community composition between the heathland soil community and ex-arable soil immediately upon abandonment is larger than between a mid-succession grassland soil and the arable community (c.f. Kardol et al. 2005, Van der Wal et al. 2006). The soil inoculum in the Stepping Stone experiment originated from a nearby field that was converted from agriculture to nature only 10 years earlier, and hence may have been functionally quite similar to that of the recipient exarable soil, thus preventing strong effect on plant community composition.

\section{Soil inoculation in practise}

Besides questions on how best to inoculate (amounts, storage etc), there are two key practical issues with soil inoculation that need to be explored. First, the level of disturbance inflicted on the donor ecosystem needs to be carefully considered. As such inoculating soil is an improvement over transplanting entire turfs, as much less material is required per unit area (Bullock 1998). In our case the donor areas are regularly disturbed by sod cutting in dry heathlands (De Graaf et al. 1998, 
Bakker and Berendse 1999) and rooting by wild boar in grasslands (Bruinderink and Hazebroek 1996) and the harvesting of donor soil has not left a long-term mark in these sites (E.R.J. Wubs, pers. observation). Whether the amount of soil needed for restoration can safely be sourced from donor habitats in future projects will need to be judged on a case by case basis.

Second, in my studies soil inocula were taken from donor ecosystems that were present within a few kilometres (Chapter 2, $3 \& 4$ ). This ensures that soil communities are selected that are appropriate for the prevailing local climate, soil type, and target vegetation. Recently, it was however suggested that it may be necessary to translocate native soils and vegetation over long distances, perhaps even across continents, in order to cope with climate-driven environmental changes (Boyer et al. 2016). However, we know relatively little about the functioning and biogeography of soil communities (Bardgett and van der Putten 2014) and it seems a large risk that such efforts might fail based on abiotic and biotic mismatches (Fahselt 2007). For instance, practical trails with mycorrhizal inoculations showed that locally sourced strains are more effective than commercial stains from elsewhere (Paluch et al. 2013, Middleton et al. 2015). Therefore, it seems prudent to use material sourced from the nearest appropriate field for the time being.

Based on Chapters 2, $3 \&$ 4, I conclude that soil inoculation can strongly influence aboveground and belowground community development. However, it is also clear that the effects and thus the impact upon nature restoration are by no means universal. For instance, topsoil removal may alter the effectiveness, presumably through its removal of the resident soil community and plant propagules. Context dependence is a the key general rule in community ecology (Lawton 1999, 2000, Fukami 2015). That is to say, the outcome of biological interactions will strongly depend on both the abiotic and biotic environmental conditions. In the case of plant-soil interactions, restoration success may depend both on the community composition of the donor and recipient community.

\section{Patch level plant-soil interactions shape plant community composition}

So far I have focussed on the consequences of manipulating plant-soil interactions at the onset of secondary succession and ensuing community developments. However, once succession is underway plants and soil biota will naturally start to interact and affect each other (Fig. 8.1). Plant-soil feedback studies have provided many new insights in these dynamics (Van der Putten et al. 2013, Bever et al. 2015), but most studies have been restricted to homogeneous soils and single 
conditioning periods (Kulmatiski et al. 2008) and spatiotemporally explicit experiments are lacking (Brandt et al. 2013, Kardol et al. 2013, Van der Putten et al. 2013). Within this thesis I have explored how spatial (Chapters $5 \& 6$ ) as well as temporal (Chapter 7) variation in soil conditioning affect the performance of mid-succession grassland species grown in monoculture or in mixed communities.

In a greenhouse experiment where plants were exposed to spatially homogeneous and heterogeneous PSFs I found that spatially heterogeneous PSFs led to lower plant performance in monocultures than expected based on homogeneous soils (Chapter 5). In addition, I directly compared the diversity of two experimental plant communities, both consisting of four grassland species, on spatially homogeneous and heterogeneous PSF soils (Chapter 6). In both communities, the diversity in the mesocosms was higher on the heterogeneous soils. On the homogeneous soils differences in plant performance were large, as some species had a disadvantage on that soil (typically own-soil), while it was more favourable to others (typically foreign-soil). In the heterogeneous soils the competitive abilities were more evenly matched, presumably because each species experienced negative PSF in at least some soil patches. As such my results support the hypothesis that PSFs drive plant diversity at small spatial scales (Petermann et al. 2008, De Kroon et al. 2012, Bever et al. 2015, Chapter 1).

\section{Conditioning diversity not heterogeneity}

In my study, there was no difference in performance and diversity of plants in fine- or coarse-grained heterogeneity treatments (Chapter $5 \& 6$ ). In addition, in the homogeneous soil that was simultaneously conditioned by four species, there was a trend that plant diversity was also higher than on homogeneous soils that were conditioned by only one species (Chapter 6). A modelling study has shown that spatial heterogeneity in PSF becomes less important for plant community composition when the spatial area from which a plant experiences soil feedback increases (Mack and Bever 2014). In my experiment the root systems of the plants were large enough to explore the whole experimental container and not confined to individual grid cells. As the roots explored both fine-grained and coarse-grained containers they were equally likely to encounter their soil-borne enemies in these treatments because the proportions of the conditioned soils were the same. Thus, based on this experiment it seems that it is the presence of conditioned soil of each species in the community rather than its precise spatial configuration that affects plant performance and thence plant diversity. How this plays out in the field 
where species have different lateral root spread and thus feedback neighbourhoods (Casper et al. 2003, Mack and Bever 2014) is an open question.

Several other studies reported that spatial heterogeneity led to higher plant performance (Brandt et al. 2013, Burns and Brandt 2014, Hendriks et al. 2015a, b), whereas in my study PSF heterogeneity instead decreased plant performance (Chapter 5). In those experiments the homogeneous controls consisted of a mix of the soils that were used to create the patches in the heterogeneous treatment. In my study, however, homogeneous controls were created by placing each conditioned soil separately in a container. I hypothesized that the strength of the feedback depends on the amount of the roots, particularly the absorptive root tissue, which is in contact with beneficial and antagonistic soil biota or their products (e.g. allelochemicals; Chapter 5 \& 6). If the soil contains foreign soil patches then the plant roots can forage there relatively unimpeded (Hendriks et al. 2015a). However, in mixed soil made up of both own and foreign soil, all of the root system will be in contact with soil-borne antagonists, thus leading to lower performance (Brandt et al. 2013, Burns and Brandt 2014, Hendriks et al. 2015a, b). However, in case the homogenous soil is made up of one conditioned soil, the performance of plants was either consistently high, when it was a foreign soil, or consistently low, when it was own soil (Chapter 5). Therefore, in my experiment the performance in heterogeneous soil was on average lower than in homogeneous soil. In line with this it was shown that on mixed soils plant performance is intermediate to the two constituent conditioned soils (Hendriks et al. 2013). The apparent difference between these studies (Brandt et al. 2013, Burns and Brandt 2014, Hendriks et al. 2015a, b, Chapter 5) is therefore in my view only methodological.

\section{Role of plant-soil feedbacks in the field}

In the soil heterogeneity experiment in the greenhouse the difference in diversity between homogeneous and heterogeneous treatments was quite small (Chapter 6). In addition, the feedback phase of this experiment was eight weeks, which is rather short when compared against the lifespan of the plants tested, which were all either biennial or perennial species. However, it is well known that competitive exclusion is a slow process and experimental grassland communities tend to lose species for many years (Booth and Grime 2003, Silvertown et al. 2006, Pierik et al. 2011). Therefore, it is likely that over time the differences in community evenness would have become larger. Naturally, these experiments provide no more than a proof of concept, demonstrating that PSFs are at least an equalizing force in competitive plant communities (sensu Chesson 2000). However, it is as yet unknown whether 
the strength of the PSFs are sufficiently strong to maintain stable plant coexistence under field conditions. There are studies that show that PSF effects observed in the lab correlate positively with relative abundance of those species in the field in grasslands (Klironomos 2002) and forests (Mangan et al. 2010), but this pattern is not universal (Reinhart 2012, Bauer et al. 2015). This suggests that PSF can be a strong force in the field, but that other factors may also overrule it.

Besides spatial interactions, the temporal development of plant-soil interactions is also key to understanding community development (Packer and Clay 2004, Kardol et al. 2013). I found that these feedback effects can carry-over across succeeding conditioning periods in complex ways (Chapter 7). A common assumption is that the longer the soil is conditioned by a particular species the stronger the resulting feedback. Some studies have found this for repeated conspecific conditioning (Mazzola 1999, Packer and Clay 2004), but my results indicate that the feedback from sequential conditioning depends strongly on the species combination that conditioned the soil (Chapter 7). Conditioning effects of one species may therefore importantly alter the effect of subsequent soil conditioning, which challenges two common assumptions in PSF models of plant communities. First, that PSF scales with conditioning biomass, and second, that current conditioning removes any prior plant-induced soil legacies (Bonanomi et al. 2005a, Eppinga et al. 2006, Fukami and Nakajima 2013). These kind of priority effects may importantly shape which species replace each other (Ejrnæs et al. 2006, Fukami 2015), but their complexity may preclude straightforward prediction of these replacements and thus the development of community composition over time (Ejrnæs et al. 2006).

What is clear is that the net effect of PSF varies across environmental conditions and species (Bezemer et al. 2006, Van der Putten et al. 2016). For instance, when limiting nutrients are added to plant communities plant alpha diversity sharply decreases (Crawley et al. 2005, Harpole et al. 2016). When soil nutrient availability increases in the soil, plant competition shifts from mainly root competition for these nutrients to competition for light aboveground (Lamb et al. 2009, Hautier et al. 2009). In addition, the strength of PSF effects is lower when nutrients are added (De Deyn et al. 2004, Manning et al. 2008, Kos et al. 2015, Chapter 7). In consequence, PSFs are likely to be a strong driver of plant community dynamics particularly under low fertility and to a much more limited extend under conditions of high soil fertility. 
In general my results (Chapter 5, $6 \& 7$ ) suggest that PSF can help maintain plant diversity at small spatial scales (Petermann et al. 2008, Pendergast et al. 2013, Bever et al. 2015). However, due to different sensitivities to PSF as well as competitive responses to abiotic conditions among species, environmental factors may affect the rate of competitive exclusion and therefore the level of local plant diversity. In the general introduction (Chapter 1), I have questioned the usefulness of the niche heterogeneity hypothesis (Tilman 1982, Silvertown 2004, Tilman 2011) for understanding plant diversity at small spatial scales. My thinking seems to echo the words of Van der Maarel and Sykes (1993), who wrote of Alvar grasslands: "We [...] question the usefulness of the niche concept and re-interpret it by stating that all species of this plant community have the same habitat niche [...]. The essential variation amongst the species is their individual ability to establish or re-establish by making use of favourable conditions appearing in microsites in an unknown, complex spatio-temporal pattern". The literature shows that spatial heterogeneity in abiotic niches does not maintain plant diversity at small spatial scales (Lundholm 2009, Tamme et al. 2010, Gazol et al. 2013), while my own experiments support the role of PSFs as the responsible driver instead (Chapter $5 \& 6$ ). Therefore, I propose that it is the relationship with the soil community that drives the opportunities for species establishment and re-establishment at small spatial scales. At larger spatial scales, those that extend beyond the level of individual interacting plants (Casper et al. 2003), abiotic conditions can be sufficiently different to allow consistent species sorting and support plant beta diversity. This new hypothesis, of the scale dependent drivers of plant diversity now requires formal testing, preferably using factorial manipulations in the field.

\section{Future directions}

In the context of this thesis I see two important areas for further research: 1) gaining a general understanding of the role of soil communities in shaping plant community dynamics in the field, and 2) identifying which players are responsible for the observed community steering and their functional roles.

It is now well established that the soil community can have important direct and indirect effects on plant community composition. Most research is based on lab and greenhouse experiments, but direct evidence from the field is mounting (De Rooij-Van der Goes et al. 1995, Bonanomi et al. 2005b, Casper and Castelli 2007, Bennett et al. 2017, Teste et al. 2017). In addition, it also clear that the strength of the plant and soil community interactions varies across environmental gradients, such as soil type (Bezemer et al. 2006). A key step is now to elucidate 
how the influence of plant-soil feedbacks over plant community dynamics and diversity plays out across these gradients (Bever et al. 2015) and in the face of other processes in the field, e.g. plant competition (Casper and Castelli 2007) and herbivory (Chesson and Kuang 2008, Veen et al. 2014). In general there is a need to bring plant-soil interaction research into the field in order to better understand its consequences in real communities and also how these interaction may change under global environmental changes (Reynolds et al. 2003, Kulmatiski and Kardol 2008, Van der Putten et al. 2013, 2016). This is a broad goal: here I will focus on a few steps that can be taken to specifically understand the role of soil communities in plant community development and restoration.

I propose that more field-based soil inoculation experiments are needed across environmental gradients. Subsequent monitoring of the soil and plant community will reveal whether 1) the community from the soil inoculum can invade the resident community and 2) whether this can alter the plant community through modified plant-soil interactions. In case this leads to strong changes it is a clear demonstration that soil communities matter for the plant community and that they could be used to steer plant community developments under those particular environmental conditions. These studies could be integrated into established globally distributed experiments, such as the Nutrient Network (Borer et al. 2014) and Drought-Net (http://drought-net.org/), which would provide a rich dataset detailing where soil communities matter for plant community dynamics right across major environmental variables such as climate, hydrology, soil type and acidity.

To make sure the effects are purely due to the introduced soil community, potential effects of plant seeds and altered abiotic conditions need to be excluded. To exclude abiotic effects these experiments could combine sterilized and live inocula (e.g. (Castle et al. 2016), although soil sterilization also affects e.g. nutrient availability (Troelstra et al. 2001). It may be more constructive to make soil suspensions and inoculate these into sterilized soil from the recipient field. This soil could then be incubated for a number of weeks or months, while preventing contamination from the air, to allow levels of microbial biomass to return to normal (Hol et al. 2015) and then be used as source material for the soil inocula. During incubation light and temperature cues may be applied to the source soil in order to stimulate seed germination (Lambers et al. 2008), which together with the soil suspension step will minimize the co-introduction of seeds with the inoculum once applied to the field. 
In addition to tests across environmental gradient, studies could address hypotheses related to the composition of both the introduced and resident community (Rillig et al. 2016a, b). For instance, given the successional changes it can be expected that soil communities from later successional stages can invade early-successional soil communities, but not vice versa. This is because tighter nutrient cycling, better defended plants, and increased predation in later successional communities may preclude early-successional soil communities from invading (Holtkamp et al. 2011, Mallon et al. 2015, Morriën et al. 2017). Questions interesting from a restoration perspective include: can the successional trajectory of mid-succession plant communities be steered or is soil inoculation only successful at the start? So far soil inoculation for nature restoration has only been tested during initial redevelopment of a site, it would be interesting to test if the same technique could be used to adjust and perhaps fine-tune community developments once succession is some years or decades underway. Finally, are disturbances, such as topsoil removal, needed in order successfully invade a resident soil community?

By sequencing the inocula as well as the recipient plots, using modern highthroughput techniques (Buée et al. 2009, Fierer et al. 2013, Ramirez et al. 2014), putative taxa which drive the observed changes in the plant community may be identified. However, PSFs may in majority be mediated by multiple interacting taxa (Hersh et al. 2012, Benítez et al. 2013, Bever et al. 2015). Exploratory network analyses may be used to resolve which actors are involved, either singly or in conjunction with others, in shaping the inoculum effects (Stopnisek et al. 2015, Van der Heijden and Hartmann 2016, Morriën et al. 2017). In analogy to the master-genes in molecular biology, network analyses could identify soil taxa that play a central role in soil processes. Alternatively, Bayesian models may be used to reduce the number of putative players. For example Hersh et al. (2012) probabilistically isolated eight out of 160 potential plant-fungal interactions, including co-infections, as causes of tree seedling mortality. A key advantage of Bayesian analyses is that prior information, for instance whether taxa are known plant pathogens, can be formally incorporated into the analysis. In this way, the role of soil-borne taxa may be identified iteratively. The taxa thus identified could then be the focal point for experimentation and may be the cornerstone for successful inocula in restoration projects. Using PSF approaches they could be cultivated under greenhouse conditions as a resource for experimental field trails, which form a necessary next step. In addition, this approach could facilitate the ex-situ cultivation of specific soil inocula for the purpose of restoration and thus alleviate pressure on potentially sensitive donor sites. 


\section{Conclusions}

Recent research has shown that plant-soil interactions are a key aspect of the natural dynamics of plant communities. Abiotic conditions, such as resource availability, and dispersal ability determine which species may occur in a given site. However, at small spatial scales differences in niche requirements are insufficient to prevent competitive exclusion. My research using experimental plant communities shows that plant-soil feedbacks contribute to the maintenance of plant species diversity at spatial scales where individual plants directly interact (Chapter 6). Soil conditioning by multiple species ensures that each species is likely to encounter its specific soil-borne enemies. As a result, plant performance is reduced when they are grown in monoculture (Chapter 5), which reduces their competitive ability and this results in a higher community diversity (Chapter 6). It is key that all species in the community conditioned part of the soil within the local neighbourhood in order to prevent one from becoming dominant. However, the spatial distribution, e.g. the grain of heterogeneity, does not affect the outcome (Chapter 5 \& 6). How individual species are affected by PSF and how these PSFs carry-over from one conditioning period to the next is, however, highly species specific (Chapter 5, $6 \& 7$ ). These insights are based on short-term experiments in the greenhouse and field based validation is now required.

Using a large-scale field experiment I showed that inoculated soil communities can alter and even steer the direction of plant community development in the field at a scale relevant for practise (Chapter 2). Furthermore, the composition of the soil inocula can be engineered to favour specific species beneficial to restoration (Chapter 3) and soil inoculation can influence soil community composition for prolonged periods of time, even decades (Chapter $2 \& 4$ ). However, soil inoculation strongly affected plant community composition in one field experiment (Chapter 2), but only for three years in another (Chapter 4). This shows that soil inoculation may not work in all environmental conditions. Therefore, more studies are needed to test the success of introducing soil communities across environmental gradients. 


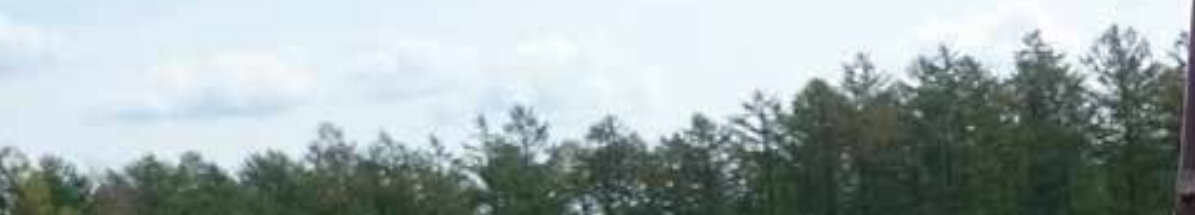
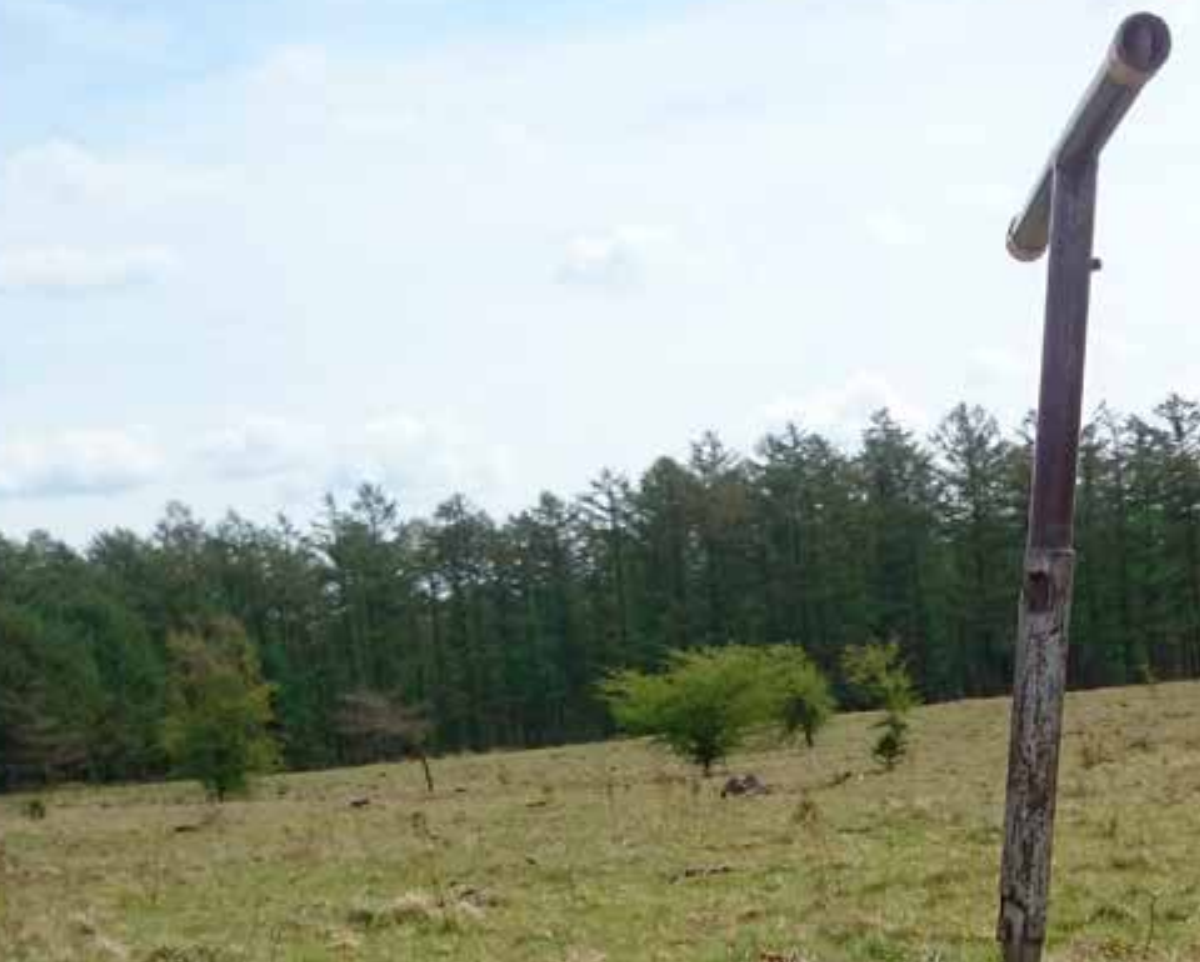

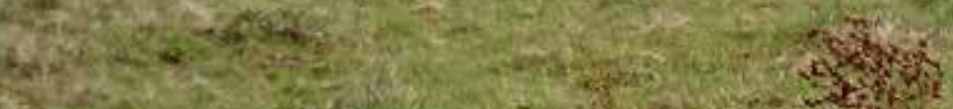

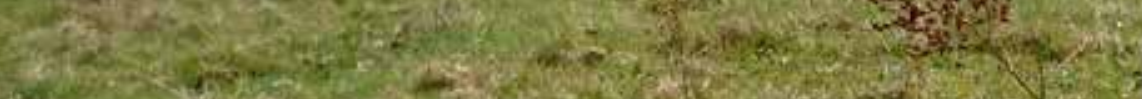

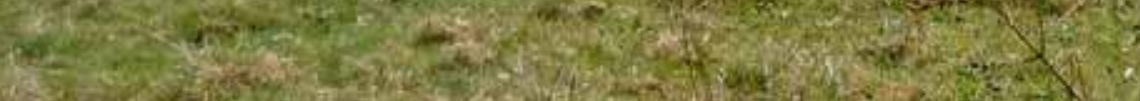

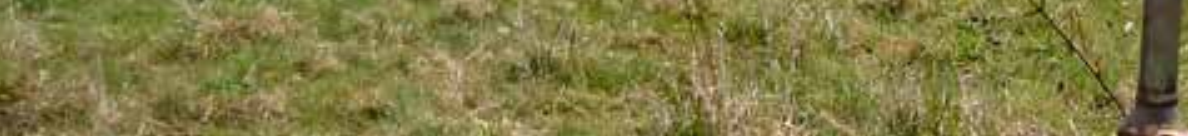
2.

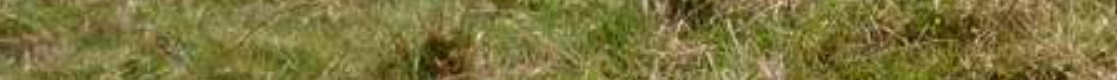

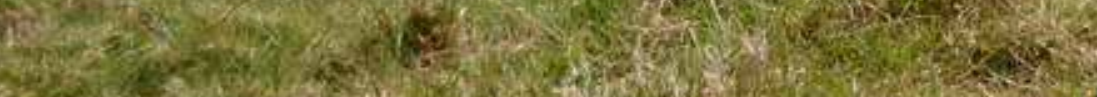

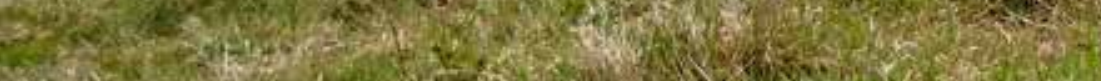
S. 13:

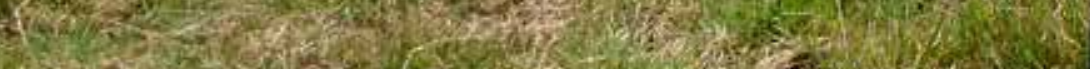

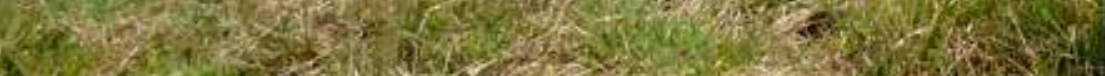
2.

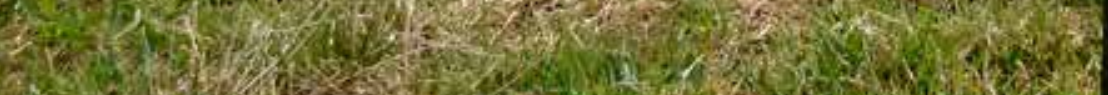

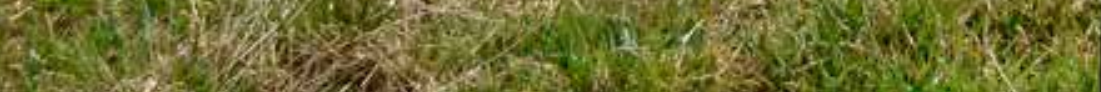

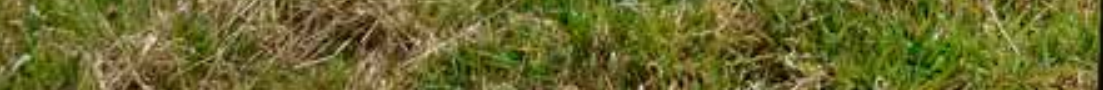

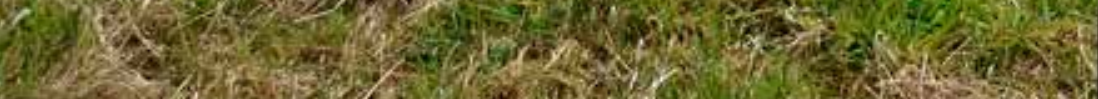

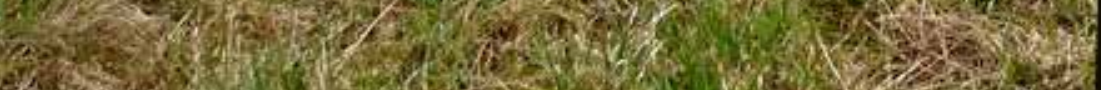
a 250.

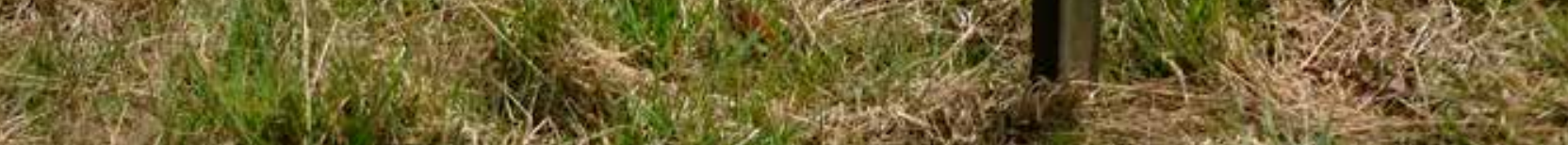

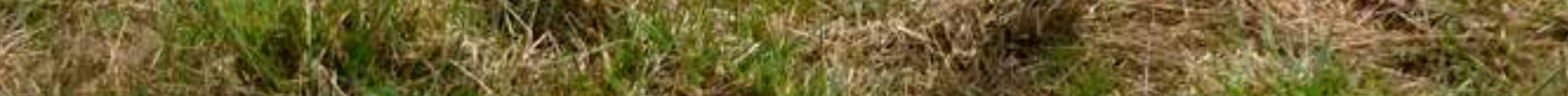
Wy - 30 .

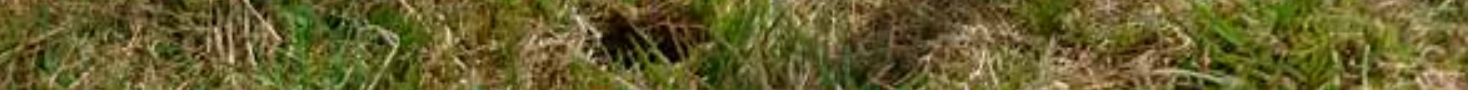




\section{References}


Abbott, K. C., J. Karst, L. A. Biederman, S. R. Borrett, A. Hastings, V. Walsh, and J. D. Bever. 2015. Spatial heterogeneity in soil microbes alters outcomes of plant competition. PLoS ONE 10:e0125788.

Adbi, H., and L. J. Williams. 2010. Contrast analysis. Page in N. Salkind, editor. Encyclopedia of Research Design. Sage, Thousand Oaks, CA, USA.

Agrios, G. N. 1978. Plant pathology. 2nd edition. Academic Press, New York, USA.

Albert, Á.-J., A. Kelemen, O. Valkó, T. Miglécz, A. Csecserits, T. Rédei, B. Deák, B. Tóthmérész, and P. Török. 2014. Secondary succession in sandy old-fields: a promising example of spontaneous grassland recovery. Applied Vegetation Science 17:214-224.

Allouche, O., M. Kalyuzhny, G. Moreno-Rueda, M. Pizarro, and R. Kadmon. 2012. Areaheterogeneity tradeoff and the diversity of ecological communities. Proceedings of the National Academy of Sciences of the United States of America 109:17495-17500.

Anacker, B. L., J. N. Klironomos, H. Maherali, K. O. Reinhart, and S. Y. Strauss. 2014. Phylogenetic conservatism in plant-soil feedback and its implications for plant abundance. Ecology Letters 17:1613-1621.

Anderson, M. J. 2001. A new method for non-parametric multivariate analysis of variance. Austral Ecology 26:32-46.

Antonsen, H., and P. A. Olsson. 2005. Relative importance of burning, mowing and species translocation in the restoration of a former boreal hayfield: responses of plant diversity and the microbial community. Journal of Applied Ecology 42:337-347.

Augspurger, C. K., and C. K. Kelly. 1984. Pathogen mortality of tropical tree seedlings: experimental studies of the effects of dispersal distance, seedling density, and light conditions. Oecologia 61:211-217.

Austin, M. P. 2013. Inconsistencies between theory and methodology: a recurrent problem in ordination studies. Journal of Vegetation Science 24:251-268.

Averett, J. M., R. A. Klips, L. E. Nave, S. D. Frey, and P. S. Curtis. 2004. Effects of soil carbon amendment on nitrogen availability and plant growth in an experimental tallgrass prairie restoration. Restoration Ecology 12:568-574.

Baer, S. G., J. M. Blair, S. L. Collins, and A. K. Knapp. 2004. Plant community responses to resource availability and heterogeneity during restoration. Oecologia 139:617-629.

Baer, S. G., D. J. Kitchen, J. M. Blair, and C. W. Rice. 2002. Changes in ecosystem structure and function along a chronosequence of restored grasslands. Ecological Applications 12:1688-1701.

Bakker, J. P., and F. Berendse. 1999. Constraints in the restoration of ecological diversity in grassland and heathland communities. Trends in Ecology \& Evolution 14:63-68.

Bakker, J. P., J. A. Elzinga, and Y. de Vries. 2002. Effects of long-term cutting in a grassland system: perspectives for restoration of plant communities on nutrient-poor soils. Applied Vegetation Science 5:107-120. 
Bardgett, R. D., W. D. Bowman, R. Kaufmann, and S. K. Schmidt. 2005. A temporal approach to linking aboveground and belowground ecology. Trends in Ecology \& Evolution 20:634-641.

Bardgett, R. D., J. C. Frankland, and J. B. Whittaker. 1993. The effects of agricultural management on the soil biota of some upland grasslands. Agriculture, Ecosystems \& Environment 45:25-45.

Bardgett, R. D., P. J. Hobbs, and Å. Frostegård. 1996. Changes in soil fungal:bacterial biomass ratios following reductions in the intensity of management of an upland grassland. Biology and Fertility of Soils 22:261-264.

Bardgett, R. D., J. L. Mawdsley, S. Edwards, P. J. Hobbs, J. S. Rodwell, and W. J. Davies. 1999. Plant species and nitrogen effects on soil biological properties of temperate upland grasslands. Functional Ecology 13:650-660.

Bardgett, R. D., L. Mommer, and F. T. De Vries. 2014. Going underground: root traits as drivers of ecosystem processes. Trends in Ecology \& Evolution 29:692-699.

Bardgett, R. D., and W. H. Van der Putten. 2014. Belowground biodiversity and ecosystem functioning. Nature 515:505-511.

Bardgett, R. D., and D. A. Wardle. 2010. Aboveground-belowground linkages. Biotic interactions, ecosystem processes and global change. Oxford University Press, Oxford, UK.

Barni, E., and C. Siniscalco. 2000. Vegetation dynamics and arbuscular mycorrhiza in oldfield successions of the western Italian Alps. Mycorrhiza 10:63-72.

Bartha, S., S. Szentes, A. Horváth, J. Házi, Z. Zimmermann, C. Molnár, I. Dancza, K. Margóczi, R. W. Pál, D. Purger, D. Schmidt, M. Óvári, C. Komoly, Z. Sutyinszki, G. Szabó, A. I. Csathó, M. Juhász, K. Penksza, and Z. Molnár. 2014. Impact of mid-successional dominant species on the diversity and progress of succession in regenerating temperate grasslands. Applied Vegetation Science 17:201-213.

Bates, D., M. Maechler, B. M. Bolker, and S. Walker. 2013. lme4: Linear mixed-effects models using Eigen and S4. http://arxiv.org/abs/1406.5823.

Bauer, J. T., K. M. L. Mack, and J. D. Bever. 2015. Plant-soil feedbacks as drivers of succession: evidence from remnant and restored tallgrass prairies. Ecosphere 6:art158.

Baxendale, C., K. H. Orwin, F. Poly, T. Pommier, and R. D. Bardgett. 2014. Are plant-soil feedback responses explained by plant traits? New Phytologist 204:408-423.

Bazzaz, F. A. 1968. Succession on abandoned fields in the Shawnee Hills, Southern Illinois. Ecology 49:924-936.

Bekker, R. M. 2009. 20 jaar ontgronden voor natuur op zandgronden. De Levende Natuur 110:9-15.

Bekker, R. M., G. L. Verweij, R. E. N. Smith, R. Reine, J. P. Bakker, and S. Schneider. 1997. Soil seed banks in European grasslands: does land use affect regeneration perspectives? Journal of Applied Ecology 34:1293-1310. 
Bell, T., R. P. Freckleton, and O. T. Lewis. 2006. Plant pathogens drive density-dependent seedling mortality in a tropical tree. Ecology Letters 9:569-574.

Benítez, M.-S., M. H. Hersh, R. Vilgalys, and J. S. Clark. 2013. Pathogen regulation of plant diversity via effective specialization. Trends in Ecology \& Evolution 28:705-711.

Benjamini, Y., and Y. Hochberg. 1995. Controlling the false discovery rate: a practical and powerful approach to multiple testing. Journal of the Royal Statistical Society B 57:289-300.

Bennett, J. A., and J. F. Cahill. 2016. Fungal effects on plant-plant interactions contribute to grassland plant abundances: evidence from the field. Journal of Ecology 104:755764.

Bennett, J. A., H. Maherali, K. O. Reinhart, Y. Lekberg, M. M. Hart, and J. Klironomos. 2017. Plant-soil feedbacks and mycorrhizal type influence temperate forest population dynamics. Science 355:181-184.

Berendse, F., M. J. M. Oomes, H. J. Altena, and W. T. Elberse. 1992. Experiments on the restoration of species-rich meadows in The Netherlands. Biological Conservation 62:59-65.

Berg, M. P., and L. Hemerik. 2004. Secondary succession of terrestrial isopod, centipede, and millipede communities in grasslands under restoration. Biology and Fertility of Soils 40:163170.

Bever, J. D. 1994. Feedback between plants and their soil communities in an old field community. Ecology 75:1965-1977.

Bever, J. D. 2003. Soil community feedback and the coexistence of competitors: conceptual frameworks and empirical tests. New Phytologist 157:465-473.

Bever, J. D., I. A. Dickie, E. Facelli, J. M. Facelli, J. Klironomos, M. Moora, M. C. Rillig, W. D. Stock, M. Tibbett, and M. Zobel. 2010. Rooting theories of plant community ecology in microbial interactions. Trends in Ecology \& Evolution 25:468-78.

Bever, J. D., S. Mangan, and H. M. Alexander. 2015. Maintenance of plant species diversity by pathogens. Annual Review of Ecology, Evolution, and Systematics 46: 305-25.

Bever, J. D., T. G. Platt, and E. R. Morton. 2012. Microbial population and community dynamics on plant roots and their feedbacks on plant communities. Annual Review of Microbiology 66:265-283.

Bever, J. D., K. M. Westover, and J. Antonovics. 1997. Incorporating the soil community into plant population dynamics: the utility of the feedback approach. Journal of Ecology 85:561-573.

Bezemer, T. M., M. T. Fountain, J. M. Barea, S. Christensen, S. C. Dekker, H. Duyts, R. Van Hal, J. A. Harvey, K. Hedlund, M. Maraun, J. Mikola, A. G. Mladenov, C. Robin, P. C. de Ruiter, S. Scheu, H. Setälä, P. Smilauer, and W. H. Van der Putten. 2010. Divergent composition but similar function of soil food webs of individual plants: plant species and community effects. Ecology 91:3027-3036. 
Bezemer, T. M., C. S. Lawson, K. Hedlund, A. R. Edwards, A. J. Brook, J. M. Igual, S. R. Mortimer, and W. H. Van Der Putten. 2006. Plant species and functional group effects on abiotic and microbial soil properties and plant-soil feedback responses in two grasslands. Journal of Ecology 94:893-904.

Bezemer, T. M., and W. H. Van der Putten. 2007. Diversity and stability in plant communities. Nature 446:E6-E7.

Bezemer, T. M., W. H. Van der Putten, H. Martens, T. F. J. Van de Voorde, P. P. J. Mulder, and O. Kostenko. 2013. Above- and below-ground herbivory effects on below-ground plant-fungus interactions and plant-soil feedback responses. Journal of Ecology 101:325-333.

Bignal, E. M., and D. I. McCracken. 1996. Low-intensity farming systems in the conservation of the countryside. Journal of Applied Ecology 33:413-424.

Blumenthal, D. M., N. R. Jordan, and M. P. Russelle. 2003. Soil carbon addition controls weeds and facilitates prairie restoration. Ecological Applications 13:605-615.

Bobbink, R., M. Hornung, and J. G. M. Roelofs. 1998. The effects of air-borne nitrogen pollutants on species diversity in natural and semi-natural European vegetation. Journal of Ecology 86:717-738.

Bonanomi, G., F. Giannino, and S. Mazzoleni. 2005a. Negative plant-soil feedback and species coexistence. Oikos 111:311-321.

Bonanomi, G., M. Rietkerk, S. C. Dekker, and S. Mazzoleni. 2005b. Negative plant-soil feedback and positive species interaction in a herbaceous plant community. Plant Ecology 181:269.

Bonet, A. 2004. Secondary succession of semi-arid Mediterranean old-fields in south-eastern Spain: insights for conservation and restoration of degraded lands. Journal of Arid Environments 56:213-233.

Bongers, T. 1988. De nematoden van Nederland. Pirola, Schoorl. Pirola, Schoorl, the Netherlands.

Bongers, T., and M. Bongers. 1998. Functional diversity of nematodes. Applied Soil Ecology 10:239-251.

Booth, R. E., and J. P. Grime. 2003. Effects of genetic impoverishment on plant community diversity. Journal of Ecology 91:721-730.

Borer, E. T., W. S. Harpole, P. B. Adler, E. M. Lind, J. L. Orrock, E. W. Seabloom, and M. D. Smith. 2014. Finding generality in ecology: a model for globally distributed experiments. Methods in Ecology and Evolution 5:65-73.

Boschker, H. T. S. 2004. Linking microbial community structure and functioning: stable isotope (13C) labeling in combination with PLFA analysis. Pages 1673-1688 in G. A. Kowalchuk, F. J. de Bruijn, I. M. Head, A. D. Akkermans, and J. D. van Elsas, editors. Molecular Microbial Ecology Manual. Springer, Dordrecht.

Box, J., M. Brown, N. Coppin, N. Hawkeswood, M. Webb, A. Hill, Q. Palmer, M. Le Duc, and P. Putwain. 2011. Experimental wet heath translocation in Dorset, England. Ecological Engineering 37:158-171. 
Boyer, S., B. S. Case, M.-C. Lefort, B. R. Waterhouse, and S. D. Wratten. 2016. Can ecosystemscale translocations mitigate the impact of climate change on terrestrial biodiversity? Promises, pitfalls, and possibilities. F1000Research 5:146.

Bradford, M. A., T. H. Jones, R. D. Bardgett, H. I. J. Black, B. Boag, M. Bonkowski, R. Cook, T. Eggers, A. C. Gange, S. J. Grayston, E. Kandeler, A. E. McCaig, J. E. Newington, J. I. Prosser, H. Setälä, P. L. Staddon, G. M. Tordoff, D. Tscherko, and J. H. Lawton. 2002. Impacts of soil faunal community composition on model grassland ecosystems. Science 298:615-8.

Bradley, D. J., G. S. Gilbert, and J. B. H. Martiny. 2008. Pathogens promote plant diversity through a compensatory response. Ecology Letters 11:461-469.

Bradshaw, A. D. 1983. The reconstruction of ecosystems. Journal of Applied Ecology 20:1-17.

Brandt, A. J., H. De Kroon, H. L. Reynolds, and J. H. Burns. 2013. Soil heterogeneity generated by plant-soil feedbacks has implications for species recruitment and coexistence. Journal of Ecology 101:277-286.

Bray, J. R., and J. T. Curtis. 1957. An ordination of the upland forest communities of southern Wisconsin. Ecological Monographs 27:326-349.

Breman, G., F. Dupuis, R. Van Ekeris, P. Hendriks, M. Hijink, T. Smeets, R. Vossebeld, J. van der Weel, and R. Weyman. 2009. Het Reemsterveld of Planken Wambuis. Onderzoek naar de plaats van cultuurhistorische landschapselementen op het Planken Wambuis. Werkgroep Landschapsgeschiedenis, Ede.

Bretfeld, G. 1999. Synopses on palaearctic collembola, volume 2: Symphypleona. Abh. Ber. Naturkundemus, Görlitz.

Brinkman, E. P., C. E. Raaijmakers, J. M. T. Bakx-Schotman, S. E. Hannula, R. H. Kemmers, W. de Boer, and W. H. van der Putten. 2012. Matgrass sward plant species benefit from soil organisms. Applied Soil Ecology 62:61-70.

Brinkman, E. P., W. H. Van der Putten, E.-J. Bakker, and K. J. F. Verhoeven. 2010. Plant-soil feedback: experimental approaches, statistical analyses and ecological interpretations. Journal of Ecology 98:1063-1073.

Brown, P. E. 1918. Soil inoculation. Iowa Agricultural Experiment Station Circulars: paper 43.

Brown, V. K., and A. C. Gange. 1989. Differential effects of above- and below-ground insect herbivory during early plant succession. Oikos 54:67-76.

Brown, V. K., and A. C. Gange. 1990. Insect herbivory below ground. Pages 1-58 in A. H. F. and A. M. M. Begon, editor. Advances in Ecological Research. Academic Press.

Brown, V. K., and A. C. Gange. 1992. Secondary plant succession: how is it modified by insect herbivory? Vegetatio 101:3-13.

Bruinderink, G. W. T. A. G., and E. Hazebroek. 1996. Wild boar (Sus scrofa scrofa L.) rooting and forest regeneration on podzolic soils in the Netherlands. Forest Ecology and Management 88:71-80. 
Buée, M., M. Reich, C. Murat, E. Morin, R. H. Nilsson, S. Uroz, and F. Martin. 2009. 454 Pyrosequencing analyses of forest soils reveal an unexpectedly high fungal diversity. New Phytologist 184:449-456.

Bullock, J. M. 1998. Community translocation in Britain: setting objectives and measuring consequences. Biological Conservation 84:199-214.

Bullock, J. M., and R. J. Pakeman. 1997. Grazing of lowland heath in England: Management methods and their effects on healthland vegetation. Biological Conservation 79:1-13.

Bulot, A., E. Provost, and T. Dutoit. 2014. A comparison of different soil transfer strategies for restoring a Mediterranean steppe after a pipeline leak (La Crau plain, South-Eastern France). Ecological Engineering 71:690-702.

Burns, J. H., and A. J. Brandt. 2014. Heterogeneity in plant-soil feedbacks and resident population dynamics affect mutual invasibility. Journal of Ecology 102:1048-1057.

Calderón, K., A. Spor, M.-C. Breuil, D. Bru, F. Bizouard, C. Violle, R. L. Barnard, and L. Philippot. 2017. Effectiveness of ecological rescue for altered soil microbial communities and functions. The ISME Journal 11:272-283.

Carbajo, V., B. den Braber, W. H. van der Putten, and G. B. De Deyn. 2011. Enhancement of late successional plants on ex-arable land by soil inoculations. PloS ONE 6:e21943.

Cardinale, B. J., J. E. Duffy, A. Gonzalez, D. U. Hooper, C. Perrings, P. Venail, A. Narwani, G. M. Mace, D. Tilman, D. A. Wardle, A. P. Kinzig, G. C. Daily, M. Loreau, J. B. Grace, A. Larigauderie, D. S. Srivastava, and S. Naeem. 2012. Biodiversity loss and its impact on humanity. Nature 486:59-67.

Casper, B. B., and J. P. Castelli. 2007. Evaluating plant-soil feedback together with competition in a serpentine grassland. Ecology Letters 10:394-400.

Casper, B. B., H. J. Schenk, and R. B. Jackson. 2003. Defining a plant's belowground zone of influence. Ecology 84:2313-2321.

Castillo, P., J. A. Navas-Cortés, D. Gomar-Tinoco, M. Di Vito, and R. M. Jiménez-Díaz. 2003. Interactions between Meloidogyne artiellia, the cereal and legume root-knot nematode, and Fusarium oxysporum f. sp. ciceris race 5 in chickpea. Phytopathology 93:1513-1523.

Castle, S. C., Y. Lekberg, D. Affleck, and C. C. Cleveland. 2016. Soil abiotic and biotic controls on plant performance during primary succession in a glacial landscape. Journal of Ecology 104:1555-1565.

Chapin, F. S., L. R. Walker, C. L. Fastie, and L. C. Sharman. 1994. Mechanisms of primary succession following geglaciation at Glacier Bay, Alaska. Ecological Monographs 64:149-175.

Chesson, P. 2000. Mechanisms of maintenance of species diversity. Annual Review of Ecology and Systematics 31:343-366.

Chesson, P., and J. J. Kuang. 2008. The interaction between predation and competition. Nature 456:235-238. 
Clay, K. 1990. Fungal endophytes of grasses. Annual Review of Ecology and Systematics 21:275297.

Clements, F. E. 1916. Plant succession: an analysis of the development of vegetation. Carnegie Institution of Washington, Washington, DC, USA.

Coleman, D. C., D. A. Crossley Jr., and P. F. Hendrix. 2004. Fundamentals of soil ecology. Academic Press, Burlington.

Comita, L. S., S. A. Queenborough, S. J. Murphy, J. L. Eck, K. Xu, M. Krishnadas, N. Beckman, and Y. Zhu. 2014. Testing predictions of the Janzen-Connell hypothesis: a meta-analysis of experimental evidence for distance- and density-dependent seed and seedling survival. Journal of Ecology 102:845-856.

Connell, J. H. 1971. On the role of natural enemies in preventing competitive exclusion in some marine animals and in rain forest trees. Pages 298-312 in P. J. Den Boer and G. R. Gradwell, editors. Dynamics of population. Pudoc, Wageningen, the Netherlands.

Cortois, R. 2015. Predictability of plant-soil feedback. PhD thesis, Wageningen University, Wageningen.

Cortois, R., and G. B. De Deyn. 2012. The curse of the black box. Plant and Soil 350:27-33.

Cramer, V. A., R. J. Hobbs, and R. J. Standish. 2008. What's new about old fields? Land abandonment and ecosystem assembly. Trends in Ecology \& Evolution 23:104-12.

Crawley, M. J. 2002. Statistical computing. An introduction to data analysis using S-Plus. John Wiley \& Sons, Chichester, UK.

Crawley, M. J., A. E. Johnston, J. Silvertown, M. Dodd, C. de Mazancourt, M. S. Heard, D. F. Henman, G. R. Edwards, and A. E. J. Huisman. 2005. Determinants of species richness in the Park Grass Experiment. The American Naturalist 165:179-192.

Davidson, D. W. 1993. The effects of herbivory and granivory on terrestrial plant succession. Oikos 68:23-35.

De Deyn, G. B., C. E. Raaijmakers, and W. H. Van der Putten. 2004. Plant community development is affected by nutrients and soil biota. Journal of Ecology 92:824-834.

De Deyn, G. B., C. E. Raaijmakers, H. R. Zoomer, M. P. Berg, P. C. De Ruiter, H. A. Verhoef, T. M. Bezemer, and W. H. van der Putten. 2003. Soil invertebrate fauna enhances grassland succession and diversity. Nature 422:711-3.

De Graaf, M. C. C., P. J. M. Verbeek, R. Bobbink, and J. G. M. Roelofs. 1998. Restoration of speciesrich dry heaths: the importance of appropriate soil conditions. Acta Botanica Neerlandica 47:89-111.

De Kroon, H., M. Hendriks, J. Van Ruijven, J. Ravenek, F. M. Padilla, E. Jongejans, E. J. W. Visser, and L. Mommer. 2012. Root responses to nutrients and soil biota: drivers of species coexistence and ecosystem productivity. Journal of Ecology 100:6-15. 
De Kroon, H., and E. Jongejans. 2016. Chance, variation and the nature of causality in ecological communities. Pages 197-214 in K. Landsman and E. van Wolde, editors. The Challenge of Chance. Springer International Publishing.

De Kroon, H., and L. Mommer. 2006. Root foraging theory put to the test. Trends in Ecology \& Evolution 21:113-116.

De Rooij-Van der Goes, P. C. E. M., B. A. M. Peters, and W.H. Van der Putten. 1998. Vertical migration of nematodes and soil-borne fungi to developing roots of Ammophila arenaria (L.) link after sand accretion. Applied Soil Ecology 10:1-10.

De Rooij-Van der Goes, P. C. E. M. 1995. The role of plant-parasitic nematodes and soil-borne fungi in the decline of Ammophila arenaria (L.) Link. New Phytologist 129:661-669.

De Rooij-Van der Goes, P. C. E. M., W. H. Van der Putten, and B. A. M. Peters. 1995. Effects of sand deposition on the interaction between Ammophila arenaria, plant-parasitic nematodes, and pathogenic fungi. Canadian Journal of Botany 73:1141-1150.

De Ruiter, P. C., A.-M. Neutel, and J. C. Moore. 1994. Modelling food webs and nutrient cycling in agro-ecosystems. Trends in Ecology \& Evolution 9:378-383.

De Ruiter, P. C., A.-M. Neutel, and J. C. Moore. 1995. Energetics, patterns of interaction strengths, and stability in real ecosystems. Science 269:1257-1260.

De Vries, F. T., J. Bloem, N. van Eekeren, L. Brusaard, and E. Hoffland. 2007. Fungal biomass in pastures increases with age and reduced N input. Soil Biology and Biochemistry 39:1620-1630.

De Vries, F. T., E. Thébault, M. Liiri, K. Birkhofer, M. A. Tsiafouli, L. Bjørnlund, H. B. Jørgensen, M. V. Brady, S. Christensen, P. C. de Ruiter, T. d'Hertefeldt, J. Frouz, K. Hedlund, L. Hemerik, W. H. G. Hol, S. Hotes, S. R. Mortimer, H. Setälä, S. P. Sgardelis, K. Uteseny, W. H. van der Putten, V. Wolters, and R. D. Bardgett. 2013. Soil food web properties explain ecosystem services across European land use systems. Proceedings of the National Academy of Sciences 110:14296-14301.

Dias, T., A. Dukes, and P. M. Antunes. 2015. Accounting for soil biotic effects on soil health and crop productivity in the design of crop rotations. Journal of the Science of Food and Agriculture 95:447-454.

Donath, T. W., S. Bissels, N. Hölzel, and A. Otte. 2007. Large scale application of diaspore transfer with plant material in restoration practice - Impact of seed and microsite limitation. Biological Conservation 138:224-234.

Duprè, C., C. J. Stevens, T. Ranke, A. Bleeker, C. Peppler-Lisback, D. J. G. Gowing, N. B. Dise, E. Dorland, R. Bobbink, and M. Diekmann. 2010. Changes in species richness and composition in European acidic grasslands over the past 70 years: the contribution of cumulative atmospheric nitrogen deposition. Global Change Biology 16:344-357.

Egler, F. E. 1954. Vegetation science concepts I. Initial floristic composition, a factor in old-field vegetation development. Vegetatio 4:412-417. 
Ehrenfeld, J. G., B. Ravit, and K. Elgersma. 2005. Feedback in the plant-soil system. Annual Review of Environment and Resources 30:75-115.

Ehrenfeld, J. G., and L. A. Toth. 1997. Restoration ecology and the ecosystem perspective. Restoration Ecology 5:307-317.

Eilts, J. A., G. G. Mittelbach, Reynolds Heather L., and K. L. Gross. 2011. Resource heterogeneity, soil fertility, and species diversity: effects of clonal species on plant communities. The American Naturalist 177:574-588.

Ejrnæs, R., H. H. Bruun, and B. J. Graae. 2006. Community assembly in experimental grasslands: suitable environment or timely arrival? Ecology 87:1225-1233.

Emam, T. 2016. Local soil, but not commercial AMF inoculum, increases native and non-native grass growth at a mine restoration site. Restoration Ecology 24:35-44.

Eppinga, M. B., M. Rietkerk, S. C. Dekker, P. C. De Ruiter, and W. H. Van der Putten. 2006. Accumulation of local pathogens: a new hypothesis to explain exotic plant invasions. Oikos 114:168-176.

Ettema, C. H., S. L. Rathbun, and D. C. Coleman. 2000. On spatiotemporal patchiness and the coexistence of five species of Chronogaster (Nematoda: Chronogasteridae) in a riparian wetland. Oecologia 125:444-452.

Ettema, C. H., and D. A. Wardle. 2002. Spatial soil ecology. Trends in Ecology \& Evolution 17:177183.

Eviner, V. T., and C. V. Hawkes. 2008. Embracing variability in the application of plant-soil interactions to the restoration of communities and ecosystems. Restoration Ecology 16:713729.

Fahselt, D. 2007. Is transplanting an effective means of preserving vegetation? Canadian Journal of Botany 85:1007-1017.

Fargione, J., C. S. Brown, and D. Tilman. 2003. Community assembly and invasion: an experimental test of neutral versus niche processes. Proceedings of the National Academy of Sciences 100:8916-8920.

Felker-Quinn, E., J. K. Bailey, and J. A. Schweitzer. 2011. Soil biota drive expression of genetic variation and development of population-specific feedbacks in an invasive plant. Ecology 92:1208-1214.

Felske, A., and A. D. L. Akkermans. 1998. Spatial homogeneity of abundant bacterial 16S rRNA molecules in grassland soils. Microbial Ecology 36:31-36.

Ferris, H., T. Bongers, and R. G. M. de Goede. 2001. A framework for soil food web diagnostics: extension of the nematode faunal analysis concept. Applied Soil Ecology 18:13-29.

Fierer, N., J. Ladau, J. C. Clemente, J. W. Leff, S. M. Owens, K. S. Pollard, R. Knight, J. A. Gilbert, and R. L. McCulley. 2013. Reconstructing the microbial diversity and function of preagricultural tallgrass prairie soils in the United States. Science 342:621-624. 
Fitzsimons, M. S., and R. M. Miller. 2010. The importance of soil microorganisms for maintaining diverse plant communities in tallgrass prairie. American Journal of Botany 97:1937-43.

Fjellberg, A. 1998. The Collembola of Fennoscandia and Denmark. Part I: Poduromorpha. Brill, Leiden.

Fjellberg, A. 2007. The Collembola of Fennoscandia and Denmark. Part II: Entomobryomorpha and Symphypleona. Brill, Leiden.

Folman, L. B., J. Postma, and J. A. Van Veen. 2001. Ecophysiological characterization of rhizosphere bacterial communities at different root locations and plant developmental stages of cucumber grown on rockwool. Microbial Ecology 42:586-597.

Foster, B. L., C. A. Murphy, K. R. Keller, T. A. Aschenbach, E. J. Questad, and K. Kindscher. 2007. Restoration of prairie community structure and ecosystem function in an abandoned hayfield: a sowing experiment. Restoration Ecology 15:652-661.

Fox, G. A., S. Negrete-Yankelevich, and V. J. Sosa. 2015. Ecological statistics: contemporary theory and application. Oxford University Press, Oxford, UK.

Fransen, B., and H. de Kroon. 2001. Long-term disadvantages of selective root placement: root proliferation and shoot biomass of two perennial grass species in a 2-year experiment. Journal of Ecology 89:711-722.

Fraser, L. H., and J. P. Grime. 1999. Interacting effects of herbivory and fertility on a synthesized plant community. Journal of Ecology 87:514-525.

Frostegård, A., and E. Bååth. 1996. The use of phospholipid fatty acid analysis to estimate bacterial and fungal biomass in soil. Biology and Fertility of Soils 22:59-65.

Frostegård, Å., A. Tunlid, and E. Bååth. 1993. Phospholipid fatty acid composition, biomass, and activity of microbial communities from two soil types experimentally exposed to different heavy metals. Applied and Environmental Microbiology 59:3605-3617.

Frouz, J., A. Toyota, O. Mudrák, V. Jílková, A. Filipová, and T. Cajthaml. 2016. Effects of soil substrate quality, microbial diversity and community composition on the plant community during primary succession. Soil Biology and Biochemistry 99:75-84.

Fukami, T. 2015. Historical contingency in community assembly: integrating niches, species pools, and priority effects. Annual Review of Ecology, Evolution, and Systematics 46:1-23.

Fukami, T., T. Martijn Bezemer, S. R. Mortimer, and W. H. Putten. 2005. Species divergence and trait convergence in experimental plant community assembly. Ecology Letters 8:1283-1290.

Fukami, T., and M. Nakajima. 2013. Complex plant-soil interactions enhance plant species diversity by delaying community convergence. Journal of Ecology 101:316-324.

Galecki, A. T., and T. Burzykowski. 2013. Linear mixed-effects models using R a step-by-step approach. Springer, New York, NY, USA.

Gardes, M., and T. D. Bruns. 1993. ITS primers with enhanced specificity for basidiomycetes application to the identification of mycorrhizae and rusts. Molecular Ecology 2:113-118. 
Gazol, A., R. Tamme, J. N. Price, I. Hiiesalu, L. Laanisto, and M. Pärtel. 2013. A negative heterogeneity-diversity relationship found in experimental grassland communities. Oecologia $173: 545-555$.

Giller, P. S. 1996. The diversity of soil communities, the "poor man's tropical rainforest." Biodiversity and Conservation 5:135-168.

Gisin, H. 1960. Collembolenfauna Europas. Museum d'Histoire Naturelle, Geneve, Switzerland.

Gleason, H. A. 1917. The structure and development of the plant association. Bulletin of the Torrey Botanical Club 43:463-481.

Grayston, S. J., S. Wang, C. D. Campbell, and A. C. Edwards. 1998. Selective influence of plant species on microbial diversity in the rhizosphere. Soil Biology and Biochemistry 30:369-378.

Green, J., and B. J. M. Bohannan. 2006. Spatial scaling of microbial biodiversity. Trends in Ecology \& Evolution 21:501-507.

Grime, J. P., and J. M. L. Mackey. 2002. The role of plasticity in resource capture by plants. Evolutionary Ecology 16:299-307.

Grime, P. J. 2001. Plant strategies, vegetation processes, and ecosystem properties. 2nd edition. John Wiley \& Sons, Chichester, UK.

Grömping, U. 2006. Relative importance for linear regression in R: the package relaimpo. Journal of Statistical Software 17:1-26.

Grubb, P. J. 1977. The maintenance of species-richness in plant communities: the importance of the regeneration niche. Biological Review 52:107-145.

Hamman, S. T., and C. V. Hawkes. 2013. Biogeochemical and microbial legacies of non-native grasses can affect restoration success. Restoration Ecology 21:58-66.

Háněl, L. 2010. An outline of soil nematode succession on abandoned fields in South Bohemia. Applied Soil Ecology 46:355-371.

Hardin, G. 1960. The competitive exclusion principle. Science 131:1292-1297.

Harpole, W. S., L. L. Sullivan, E. M. Lind, J. Firn, P. B. Adler, E. T. Borer, J. Chase, P. A. Fay, Y. Hautier, H. Hillebrand, A. S. MacDougall, E. W. Seabloom, R. Williams, J. D. Bakker, M. W. Cadotte, E. J. Chaneton, C. Chu, E. E. Cleland, C. D’Antonio, K. F. Davies, D. S. Gruner, N. Hagenah, K. Kirkman, J. M. H. Knops, K. J. La Pierre, R. L. McCulley, J. L. Moore, J. W. Morgan, S. M. Prober, A. C. Risch, M. Schuetz, C. J. Stevens, and P. D. Wragg. 2016. Addition of multiple limiting resources reduces grassland diversity. Nature 537:93-96.

Harris, J. 2009. Soil microbial communities and restoration ecology: facilitators or followers? Science 325:573-4.

Hautier, Y., P. A. Niklaus, and A. Hector. 2009. Competition for light causes plant biodiversity loss after eutrophication. Science 324:636-638.

Hawkes, C. V., S. N. Kivlin, J. Du, and V. T. Eviner. 2013. The temporal development and additivity of plant-soil feedback in perennial grasses. Plant and Soil 369:141-150. 
Hedlund, K., I. Santa Regina, W. H. Van der Putten, J. Lepš, T. Díaz, G. W. Korthals, S. Lavorel, V. K. Brown, D. Gormsen, S. R. Mortimer, C. Rodríguez Barrueco, J. Roy, P. Smilauer, M. Smilauerová, and C. Van Dijk. 2003. Plant species diversity, plant biomass and responses of the soil community on abandoned land across Europe: idiosyncracy or above-belowground time lags. Oikos 103:45-58.

Helgason, T., T. J. Daniell, R. Husband, A. H. Fitter, and J. P. W. Young. 1998. Ploughing up the wood-wide web? Nature 394:431-431.

Hendriks, M., L. Mommer, H. De Caluwe, A. E. Smit-Tiekstra, W. H. Van der Putten, and H. De Kroon. 2013. Independent variations of plant and soil mixtures reveal soil feedback effects on plant community overyielding. Journal of Ecology 101:287-297.

Hendriks, M., J. M. Ravenek, A. E. Smit-Tiekstra, J. W. Van der Paauw, H. De Caluwe, W. H. Van der Putten, H. De Kroon, and L. Mommer. 2015a. Spatial heterogeneity of plant-soil feedback affects root interactions and interspecific competition. New Phytologist 207:830-840.

Hendriks, M., E. J. W. Visser, I. G. S. Visschers, B. H. J. Aarts, H. De Caluwe, A. E. Smit-Tiekstra, W. H. Van der Putten, H. De Kroon, and L. Mommer. 2015b. Root responses of grassland species to spatial heterogeneity of plant-soil feedback. Functional Ecology 29:177-186.

Herben, T., F. Krahulec, V. Hadincová, and M. Kováf ova. 1993. Small-scale spatial dynamics of plant species in a grassland community over six years. Journal of Vegetation Science 4:171-178.

Hersh, M. H., R. Vilgalys, and J. S. Clark. 2012. Evaluating the impacts of multiple generalist fungal pathogens on temperate tree seedling survival. Ecology 93:511-520.

Hille Ris Lambers, J., J. S. Clark, and B. Beckage. 2002. Density-dependent mortality and the latitudinal gradient in species diversity. Nature 417:732-5.

Hobbs, R. J., and J. A. Harris. 2001. Restoration ecology: repairing the Earth's ecosystems in the new millennium. Restoration Ecology 9:239-246.

Hoeksema, J. D., V. B. Chaudhary, C. A. Gehring, N. C. Johnson, J. Karst, R. T. Koide, A. Pringle, C. Zabinski, J. D. Bever, J. C. Moore, G. W. T. Wilson, J. N. Klironomos, and J. Umbanhowar. 2010. A meta-analysis of context-dependency in plant response to inoculation with mycorrhizal fungi. Ecology letters 13:394-407.

Hol, W. H. G., W. De Boer, M. De Hollander, E. E. Kuramae, A. Meisner, and W. H. Van der Putten. 2015. Context dependency and saturating effects of loss of rare soil microbes on plant productivity. Frontiers in Plant Science 6:485.

Holtkamp, R., P. Kardol, A. Van der Wal, S. C. Dekker, W. H. Van der Putten, and P. C. De Ruiter. 2008. Soil food web structure during ecosystem development after land abandonment. Applied Soil Ecology 39:23-34.

Holtkamp, R., A. Van der Wal, P. Kardol, W. H. Van der Putten, P. C. De Ruiter, and S. C. Dekker. 2011. Modelling $\mathrm{C}$ and $\mathrm{N}$ mineralisation in soil food webs during secondary succession on exarable land. Soil Biology and Biochemistry 43:251-260. 
Horn, H. S. 1974. The ecology of secondary succession. Annual Review of Ecology and Systematics 5:25-37.

Hothorn, T., F. Bretz, and P. Westfall. 2008. Simultaneous inference in general parametric models. Biometrical Journal 50:346-363.

Hutchings, M. J., and K. D. Booth. 1996. Studies on the feasibility of re-creating chalk grassland vegetation on ex-arable land. I. The potential roles of the seed bank and the seed rain. Journal of Applied Ecology 33:1171-1181.

Hutchings, M. J., E. A. John, and D. K. Wijesinghe. 2003. Toward understanding the consequences of soil heterogeneity for plant populations and communities. Ecology 84:2322-2334.

Hutchinson, G. E. 1959. Homage to Santa Rosalia or why are there so many kinds of animals? American Naturalist 93:145-159.

Hutchinson, G. E. 1961. The paradox of the plankton. American Naturalist 95:137-145.

Isbell, F., D. Tilman, S. Polasky, S. Binder, and P. Hawthorne. 2013. Low biodiversity state persists two decades after cessation of nutrient enrichment. Ecology Letters 16:454-460.

Jackson, S. T., and R. J. Hobbs. 2009. Ecological restoration in the light of ecological history. Science 325:567-569.

Jakobsson, A., and O. Eriksson. 2000. A comparative study of seed number, seed size, seedling size and recruitment in grassland plants. Oikos 88:494-502.

James, T. Y., F. Kauff, C. L. Schoch, P. B. Matheny, V. Hofstetter, C. J. Cox, G. Celio, C. Gueidan, E. Fraker, J. Miadlikowska, H. T. Lumbsch, A. Rauhut, V. Reeb, A. E. Arnold, A. Amtoft, J. E. Stajich, K. Hosaka, G.-H. Sung, D. Johnson, B. O’Rourke, M. Crockett, M. Binder, J. M. Curtis, J. C. Slot, Z. Wang, A. W. Wilson, A. Schüssler, J. E. Longcore, K. O’Donnell, S. Mozley-Standridge, D. Porter, P. M. Letcher, M. J. Powell, J. W. Taylor, M. M. White, G. W. Griffith, D. R. Davies, R. A. Humber, J. B. Morton, J. Sugiyama, A. Y. Rossman, J. D. Rogers, D. H. Pfister, D. Hewitt, K. Hansen, S. Hambleton, R. A. Shoemaker, J. Kohlmeyer, B. Volkmann-Kohlmeyer, R. A. Spotts, M. Serdani, P. W. Crous, K. W. Hughes, K. Matsuura, E. Langer, G. Langer, W. A. Untereiner, R. Lücking, B. Büdel, D. M. Geiser, A. Aptroot, P. Diederich, I. Schmitt, M. Schultz, R. Yahr, D. S. Hibbett, F. Lutzoni, D. J. McLaughlin, J. W. Spatafora, and R. Vilgalys. 2006. Reconstructing the early evolution of Fungi using a six-gene phylogeny. Nature 443:818-22.

Janos, D. P. 1980. Mycorrhizae influence tropical succession. Biotropica 12:56-64.

Janzen, D. H. 1970. Herbivores and the number of tree species in tropical forests. American Naturalist 104:501-528.

Jarosz, A. M., and A. L. Davelos. 1995. Effects of disease in wild plant populations and the evolution of pathogen aggressiveness. New Phytologist 129:371-387.

Jaunatre, R., E. Buisson, and T. Dutoit. 2014. Topsoil removal improves various restoration treatments of a Mediterranean steppe (La Crau, southeast France). Applied Vegetation Science $17: 236-245$. 
Jing, J., T. M. Bezemer, and W. H. van der Putten. 2015. Interspecific competition of early successional plant species in ex-arable fields as influenced by plant-soil feedback. Basic and Applied Ecology 16:112-119.

Johnson, D. J., W. T. Beaulieu, J. D. Bever, and K. Clay. 2012. Conspecific negative density dependence and forest diversity. Science 336:904-7.

Johnson, M. P., and D. S. Simberloff. 1974. Environmental determinants of island species numbers in the British Isles. Journal of Biogeography 1:149-154.

Johnson, N. C. 2010. Resource stoichiometry elucidates the structure and function of arbuscular mycorrhizas across scales. New Phytologist 185:631-647.

Johnson, N. C., D. R. Zak, D. Tilman, and F. L. Pfleger. 1991. Dynamics of vesicular-arbuscular mycorrhizae during old field succession. Oecologia 86:349-358.

Kardol, P. 2007. Plant and soil community assembly in secondary succession on ex-arable land. Fundamental and applied approaches. PhD-thesis, Wageningen University, Wageningen.

Kardol, P., T. M. Bezemer, and W. H. Van der Putten. 2006. Temporal variation in plant-soil feedback controls succession. Ecology Letters 9:1080-1088.

Kardol, P., T. M. Bezemer, and W. H. Van der Putten. 2009. Soil organism and plant introductions in restoration of species-rich grassland communities. Restoration Ecology 17:258-269.

Kardol, P., T. M. Bezemer, A. Van der Wal, and W. H. Van der Putten. 2005. Successional trajectories of soil nematode and plant communities in a chronosequence of ex-arable lands. Biological Conservation 126:317-327.

Kardol, P., N. J. Cornips, M. M. L. Van Kempen, J. M. T. Bakx-Schotman, and W. H. Van der Putten. 2007. Microbe-mediated plant-soil feedback causes historical contingency effects in plant community assembly. Ecological Monographs 77:147-162.

Kardol, P., G. B. De Deyn, E. Laliberté, P. Mariotte, and C. V. Hawkes. 2013. Biotic plant-soil feedbacks across temporal scales. Journal of Ecology 101:309-315.

Kardol, P., A. Van der Wal, T. M. Bezemer, W. de Boer, H. Duyts, R. Holtkamp, and W. H. Van der Putten. 2008. Restoration of species-rich grasslands on ex-arable land: seed addition outweighs soil fertility reduction. Biological Conservation 141:2208-2217.

Kardol, P., and D. A. Wardle. 2010. How understanding aboveground-belowground linkages can assist restoration ecology. Trends in Ecology \& Evolution 25:670-679.

Kiehl, K., A. Kirmer, T. W. Donath, L. Rasran, and N. Hölzel. 2010. Species introduction in restoration projects - Evaluation of different techniques for the establishment of semi-natural grasslands in Central and Northwestern Europe. Basic and Applied Ecology 11:285-299.

Kiers, E. T., T. M. Palmer, A. R. Ives, J. F. Bruno, and J. L. Bronstein. 2010. Mutualisms in a changing world: an evolutionary perspective. Ecology Letters 13:1459-1474.

Klironomos, J. N. 2002. Feedback with soil biota contributes to plant rarity and invasiveness in communities. Nature 417:67-70. 
Korthals, G. W., P. Smilauer, C. Van Dijk, and W. H. Van der Putten. 2001. Linking aboveand below-ground biodiversity: abundance and trophic complexity in soil as a response to experimental plant communities on abandoned arable land. Functional Ecology 15:506-514.

Kos, M., M. A. B. Tuijl, J. de Roo, P. P. J. Mulder, and T. M. Bezemer. 2015a. Plant-soil feedback effects on plant quality and performance of an aboveground herbivore interact with fertilisation. Oikos 124:658-667.

Kos, M., M. A. B. Tuijl, J. de Roo, P. P. J. Mulder, and T. M. Bezemer. 2015b. Species-specific plantsoil feedback effects on above-ground plant-insect interactions. Journal of Ecology 103:904914.

Kos, M., J. Veendrick, and T. M. Bezemer. 2013. Local variation in conspecific plant density influences plant-soil feedback in a natural grassland. Basic and Applied Ecology 14:506-514.

Kostenko, O., T. F. J. Van de Voorde, P. P. J. Mulder, W. H. Van der Putten, and T. M. Bezemer. 2012. Legacy effects of aboveground-belowground interactions. Ecology Letters 15:813-821.

Kull, K., and M. Zobel. 1991. High species richness in an Estonian wooded meadow. Journal of Vegetation Science 2:715-718.

Kulmatiski, A., and K. H. Beard. 2011. Long-term plant growth legacies overwhelm short-term plant growth effects on soil microbial community structure. Soil Biology and Biochemistry 43:823-830.

Kulmatiski, A., K. H. Beard, and J. M. Stark. 2006. Soil history as a primary control on plant invasion in abandoned agricultural fields. Journal of Applied Ecology 43:868-876.

Kulmatiski, A., K. H. Beard, J. R. Stevens, and S. M. Cobbold. 2008. Plant-soil feedbacks: a metaanalytical review. Ecology Letters 11:980-992.

Kulmatiski, A., and P. Kardol. 2008. Getting plant-soil feedback out of the greenhouse: experimental and conceptual approaches. Pages 449-472 in U. Lüttige, W. Beyschlag, and J. Murata, editors. Progress in Botany Vol. 69. Springer-Verlag, Berlin Heidelberg.

Laird, R. A., and B. S. Schamp. 2006. Competitive intransitivity promotes species coexistence. The American Naturalist 168:182-193.

Lamb, E. G., S. W. Kembel, and J. F. Cahill. 2009. Shoot, but not root, competition reduces community diversity in experimental mesocosms. Journal of Ecology 97:155-163.

Lambers, H., F. S. Chapin, and T. L. Pons. 2008. Plant Physiological Ecology. 2nd edition. Springer, New York, USA.

Lavorel, S., and E. Garnier. 2002. Predicting changes in community composition and ecosystem functioning from plant traits: revisiting the Holy Grail. Functional Ecology 16:545-556.

Lawton, J. H. 1999. Are there general laws in ecology? Oikos 84:177-192.

Lawton, J. H. 2000. Community ecology in a changing world. Ecology Institute, Oldendorff/Luhe, Germany.

Legendre, P., and L. F. J. Legendre. 2012. Numerical ecology. 3rd edition. Elsevier, Amsterdam, the Netherlands. 
Lemmermeyer, S., L. Lörcher, M. Van Kleunen, and W. Dawson. 2015. Testing the plant growthdefense hypothesis belowground: do faster-growing herbaceous plant species suffer more negative effects from soil biota than slower-growing ones? The American Naturalist 186:264-271.

Lennon, J. T., and S. E. Jones. 2011. Microbial seed banks: the ecological and evolutionary implications of dormancy. Nature Reviews Microbiology 9:119-130.

Lenth, R. 2015. Ismeans: Least-Squares Means. R package version 2.20-23. https://CRAN.R-project. org/package $=1$ smeans.

Leoni, C., M. De Vries, C. J. F. Ter Braak, A. H. C. Van Bruggen, and W. A. H. Rossing. 2013. Fusarium oxysporum f.sp. cepae dynamics: in-plant multiplication and crop sequence simulations. European Journal of Plant Pathology 137:545-561.

Letten, A. D., P.-J. Ke, and T. Fukami. 2016. Linking modern coexistence theory and contemporary niche theory. Ecological Monographs: in press (doi: 10.1002/ecm.1242).

Liu, Y., S. Yu, Z.-P. Xie, and C. Staehelin. 2012. Analysis of a negative plant-soil feedback in a subtropical monsoon forest. Journal of Ecology 100:1019-1028.

Lockwood, J. L., and S. L. Pimm. 1999. When does restoration succeed? Pages 363-392 in E. Wether and P. A. Keddy, editors. Ecological assembly rules: perspectives, advances, retreats. Cambridge University Press, Cambridge, UK.

Lugtenberg, B., and F. Kamilova. 2009. Plant-growth-promoting rhizobacteria. Annual Review of Microbiology 63:541-556.

Lundberg, D. S., S. L. Lebeis, S. H. Paredes, S. Yourstone, J. Gehring, S. Malfatti, J. Tremblay, A. Engelbrektson, V. Kunin, T. G. del Rio, R. C. Edgar, T. Eickhorst, R. E. Ley, P. Hugenholtz, S. G. Tringe, and J. L. Dangl. 2012. Defining the core Arabidopsis thaliana root microbiome. Nature 488:86-90.

Lundholm, J. T. 2009. Plant species diversity and environmental heterogeneity: spatial scale and competing hypotheses. Journal of Vegetation Science 20:377-391.

MacArthur, R. H. 1972. Geographical ecology. Princeton University Press, Princeton, NJ, USA.

Mack, K. M. L., and J. D. Bever. 2014. Coexistence and relative abundance in plant communities are determined by feedbacks when the scale of feedback and dispersal is local. Journal of Ecology 102:1195-1201.

Mallon, C. A., J. D. Van Elsas, and J. F. Salles. 2015. Microbial invasions: the process, patterns, and mechanisms. Trends in Microbiology 23:719-729.

Malý, S., G. W. Korthals, C. Van Dijk, W. H. Van der Putten, and W. De Boer. 2000. Effect of vegetation manipulation of abandoned arable land on soil microbial properties. Biology and Fertility of Soils 31:121-127.

Mangan, S. A., S. A. Schnitzer, E. A. Herre, K. M. L. Mack, M. C. Valencia, E. I. Sanchez, and J. D. Bever. 2010. Negative plant-soil feedback predicts tree-species relative abundance in a tropical forest. Nature 466:752-755. 
Manning, P., S. A. Morrison, M. Bonkowski, and R. D. Bardgett. 2008. Nitrogen enrichment modifies plant community structure via changes to plant-soil feedback. Oecologia 157:661673.

Marrs, R. H. 1993. Soil fertility and nature conservation in Europe: theoretical considerations and practical management solutions. Pages 241-300 in A. H. Fitter and M. Begon, editors. Advances in Ecological Research. Academic Press, London, UK.

Marrs, R. H. 2016. Soil microbes call the shots. Nature Plants 2:16117.

Mazzola, M. 1999. Transformation of soil microbial community structure and Rhizoctoniasuppressive potential in response to apple roots. Phytopathology 89:920-927.

Mazzoleni, S., G. Bonanomi, G. Incerti, M. L. Chiusano, P. Termolino, A. Mingo, M. Senatore, F. Giannino, F. Cartenì, M. Rietkerk, and V. Lanzotti. 2015. Inhibitory and toxic effects of extracellular self-DNA in litter: a mechanism for negative plant-soil feedbacks? New Phytologist 205:1195-1210.

McLauchlan, K. 2006. The nature and longevity of agricultural impacts on soil carbon and nutrients: a review. Ecosystems 9:1364-1382.

Mehrabi, Z., T. Bell, and O. T. Lewis. 2015. Plant-soil feedbacks from 30-year family-specific soil cultures: phylogeny, soil chemistry and plant life stage. Ecology and Evolution 5:2333-2339.

Mehrabi, Z., and S. L. Tuck. 2015. Relatedness is a poor predictor of negative plant-soil feedbacks. New Phytologist 205:1071-1075.

Middleton, E. L., and J. D. Bever. 2012. Inoculation with a native soil community advances succession in a grassland restoration. Restoration Ecology 20:218-226.

Middleton, E. L., S. Richardson, L. Koziol, C. E. Palmer, Z. Yermakov, J. A. Henning, P. A. Schultz, and J. D. Bever. 2015. Locally adapted arbuscular mycorrhizal fungi improve vigor and resistance to herbivory of native prairie plant species. Ecosphere 6:276.

Miller, S. L., P. Torres, and T. M. McClean. 1994. Persistence of Basidiospores and sclerotia of ectomycorrhizal fungi and Morchella in soil. Mycologia 86:89-95.

Mitchell, C. E., D. Tilman, and J. V. Groth. 2002. Effects of grassland plant species diversity, abundance, and composition on foliar fungal disease. Ecology 83:1713-1726.

Mitchell, R. J., M. H. D. Auld, M. G. Le Duc, and M. H. Robert. 2000. Ecosystem stability and resilience: a review of their relevance for the conservation management of lowland heaths. Perspectives in Plant Ecology, Evolution and Systematics 3:142-160.

Molofsky, J., J. D. Bever, J. Antonovics, and T. J. Newman. 2002. Negative frequency-dependence and the importance of spatial scale. Ecology 83:21-27.

Moore, J. C., K. McCann, H. Setälä, and P. C. De Ruiter. 2003. Top down is bottom up; does predation in the rhizosphere regulate aboveground dynamics? Ecology 84:846-857.

Morriën, E., S. E. Hannula, L. B. Snoek, N. R. Helmsing, H. Zweers, M. De Hollander, R. Luján Soto, M.-L. Bouffaud, M. Buée, W. Dimmers, H. Duyts, S. Geisen, M. Girlanda, R. I. Griffiths, H.-B. Jørgensen, J. Jensen, P. Plassart, D. Redecker, R. M. Schmelz, O. Schmidt, 
B. C. Thomson, E. Tisserant, S. Uroz, A. Winding, M. J. Bailey, M. Bonkowski, J. H. Faber, F. Martin, P. Lemanceau, W. De Boer, J. A. Van Veen, and W. H. Van der Putten. 2017. Soil networks become more connected and take up more carbon as nature restoration progresses. Nature Communications 8:14349.

Morris, W. F., R. A. Hufbauer, A. A. Agrawal, J. D. Bever, V. A. Borowicz, G. S. Gilbert, J. L. Maron, C. E. Mitchell, I. M. Parker, A. G. Power, M. E. Torchin, and D. P. Vázquez. 2007. Direct and interactive effects of enemies and mutualists on plant performance: a meta-analysis. Ecology 88:1021-1029.

Mueller, U. G., and J. L. Sachs. 2015. Engineering microbiomes to improve plant and animal health. Trends in Microbiology 23:606-617.

Muller, S., T. Dutoit, D. Alard, and F. Grévilliot. 1998. Restoration and rehabilitation of species-rich grassland ecosystems in France: a review. Restoration Ecology 6:94-101.

Neher, D. A. 2010. Ecology of plant and free-living Nematodes in natural and agricultural Soil. Annual Review of Phytopathology 48:371-394.

Neutel, A.-M., J. A. P. Heesterbeek, J. van de Koppel, G. Hoenderboom, A. Vos, C. Kaldeway, F. Berendse, and P. C. de Ruiter. 2007. Reconciling complexity with stability in naturally assembling food webs. Nature 449:599-602.

Ngosong, C., E. Gabriel, and L. Ruess. 2012. Use of the signature fatty acid 16:1囚5 as a tool to determine the distribution of arbuscular mycorrhizal fungi in soil. Journal of Lipids 2012:e236807.

Oksanen, J., F. G. Blanchet, R. Kindt, P. Legendre, P. R. Minchin, R. B. O’Hara, G. L. Simpson, P. Solymos, M. H. M. Stevens, and H. H. Wagner. 2016. vegan: community ecology package. R package version 2.4-1. http://CRAN.R-project.org/package=vegan.

Olff, H., F. W. M. Vera, J. Bokdam, E. S. Bakker, J. M. Gleichman, K. de Maeyer, and R. Smit. 1999. Shifting mosaics in grazed woodlands driven by the alternation of plant facilitation and competition. Plant Biology 1:127-137.

Olsen, S. R., C. V. Cole, F. S. Watanabe, and L. A. Deans. 1954. Estimation of available phosphorus in soils by extraction with sodium bicarbonate. USDA Circular 139:1-19.

Oostenbrink, M. 1960. Estimating nematode populations by some selected methods. Pages 85-102 in N. J. Sasser and W. R. Jenkins, editors. Nematology. University of North Carolina Press, Chapel Hill, NC, USA.

Packer, A., and K. Clay. 2000. Soil pathogens and spatial patterns of seedling mortality in a temperate tree. Nature 404:278-281.

Packer, A., and K. Clay. 2003. Soil pathogens and Prunus serotina seedling and sapling growth near conspecific trees. Ecology 84:108-119.

Packer, A., and K. Clay. 2004. Development of negative feedback during successive growth cycles of black cherry. Proceedings of the Royal Society of London B: Biological Sciences 271:317-324. 
Paluch, E. C., M. A. Thomsen, and T. J. Volk. 2013. Effects of resident soil fungi and land use history outweigh those of commercial mycorrhizal inocula: testing a restoration strategy in unsterilized soil. Restoration Ecology 21:380-389.

Panke-Buisse, K., A. C. Poole, J. K. Goodrich, R. E. Ley, and J. Kao-Kniffin. 2015. Selection on soil microbiomes reveals reproducible impacts on plant function. The ISME Journal 9:980-989.

Pärtel, M., R. Kalamees, M. Zobel, and E. Rosén. 1998. Restoration of species-rich limestone grassland communities from overgrown land: the importance of propagule availability. Ecological Engineering 10:275-286.

Pendergast, T. H., D. J. Burke, and W. P. Carson. 2013. Belowground biotic complexity drives aboveground dynamics: a test of the soil community feedback model. New Phytologist 197:1300-1310.

Perring, M. P., R. J. Standish, J. N. Price, M. D. Craig, T. E. Erickson, K. X. Ruthrof, A. S. Whiteley, L. E. Valentine, and R. J. Hobbs. 2015. Advances in restoration ecology: rising to the challenges of the coming decades. Ecosphere 6:art131.

Petermann, J. S., A. J. F. Fergus, L. A. Turnbull, and B. Schmid. 2008. Janzen-Connell effects are widespread and strong enough to maintain diversity in grasslands. Ecology 89:2399-2406.

Petraitis, P. S. 1979. Competitive Networks and Measures of Intransitivity. The American Naturalist 114:921-925.

Philippot, L., J. M. Raaijmakers, P. Lemanceau, and W. H. Van der Putten. 2013. Going back to the roots: the microbial ecology of the rhizosphere. Nature Reviews Microbiology 11:789-799.

Pickett, S. T. A. 1982. Population patterns through twenty years of oldfield succession. Vegetatio 49:45-59.

Pielou, E. C. 1966. The measurement of diversity in different types of biological collections. Journal of Theoretical Biology 13:131-144.

Pierik, M., J. van Ruijven, T. M. Bezemer, R. H. E. M. Geerts, and F. Berendse. 2011. Recovery of plant species richness during long-term fertilization of a species-rich grassland. Ecology 92:1393-1398.

Pinheiro, J. C., and D. M. Bates. 2000. Mixed-Effects Models in S and S-PLUS. Springer Verlag, New York.

Pinheiro, J. C., D. M. Bates, S. DebRoy, D. Sarkar, and R Development Core Team. 2016. nlme: Linear and Nonlinear Mixed Effects Models. http://CRAN.R-project.org/package=nlme.

Prach, K., R. Marrs, P. Pyšsek, and R. Van Diggelen. 2007. Manipulation of succession. Pages 121149 in L. R. Walker, J. Walker, and R. J. Hobbs, editors. Linking restoration and ecological succession. Springer, New York, USA.

Prach, K., K. Řehounková, K. Lencová, A. Jírová, P. Konvalinková, O. Mudrák, V. Študent, Z. Vaněček, L. Tichý, P. Petř́ík, P. Šmilauer, and P. Pyšek. 2014. Vegetation succession in restoration of disturbed sites in Central Europe: the direction of succession and species richness across 19 seres. Applied Vegetation Science 17:193-200. 
Pywell, R. F., J. M. Bullock, A. Hopkins, K. J. Walker, T. H. Sparks, M. J. W. Burke, and S. Peel. 2002. Restoration of species-rich grassland on arable land: assessing the limiting processes using a multi-site experiment. Journal of Applied Ecology 39:294-309.

Pywell, R. F., J. M. Bullock, J. B. Tallowin, K. J. Walker, E. A. Warman, and G. Masters. 2007. Enhancing diversity of species-poor grasslands: an experimental assessment of multiple constraints. Journal of Applied Ecology 44:81-94.

Pywell, R. F., W. R. Meek, N. R. Webb, P. D. Putwain, and J. M. Bullock. 2011. Long-term heathland restoration on former grassland: The results of a 17-year experiment. Biological Conservation 144:1602-1609.

R Core Team. 2016. R: A language and environment for statistical computing. R Foundation for Statistical Computing, Vienna, Austria.

Raaijmakers, J. M., and M. Mazzola. 2016. Soil immune responses. Science 352:1392-1393.

Raaijmakers, J. M., T. C. Paulitz, C. Steinberg, C. Alabouvette, and Y. Moënne-Loccoz. 2009. The rhizosphere: a playground and battlefield for soilborne pathogens and beneficial microorganisms. Plant and Soil 321:341-361.

Ramirez, K. S., J. M. Craine, and N. Fierer. 2012. Consistent effects of nitrogen amendments on soil microbial communities and processes across biomes. Global Change Biology 18:1918-1927.

Ramirez, K. S., J. W. Leff, A. Barberán, S. T. Bates, J. Betley, T. W. Crowther, E. F. Kelly, E. E. Oldfield, E. A. Shaw, C. Steenbock, M. A. Bradford, D. H. Wall, and N. Fierer. 2014. Biogeographic patterns in below-ground diversity in New York City's Central Park are similar to those observed globally. Proceedings of the Royal Society of London B: Biological Sciences 281:20141988.

Rasmann, S., T. L. Bauerle, K. Poveda, and R. Vannette. 2011. Predicting root defence against herbivores during succession. Functional Ecology 25:368-379.

Redhead, J. W., J. Sheail, J. M. Bullock, A. Ferreruela, K. J. Walker, and R. F. Pywell. 2014. The natural regeneration of calcareous grassland at a landscape scale: 150 years of plant community re-assembly on Salisbury Plain, UK. Applied Vegetation Science 17:408-418.

Reinhart, K. O. 2012. The organization of plant communities: negative plant-soil feedbacks and semiarid grasslands. Ecology 93:2377-2385.

Revilla, T. A., G. F. Veen, M. B. Eppinga, and F. J. Weissing. 2013. Plant-soil feedbacks and the coexistence of competing plants. Theoretical Ecology 6:99-113.

Reynolds, H. L., and K. A. Haubensak. 2009. Soil fertility, heterogeneity, and microbes: towards an integrated understanding of grassland structure and dynamics. Applied Vegetation Science $12: 33-44$.

Reynolds, H. L., A. Packer, J. D. Bever, and K. Clay. 2003. Grassroots ecology: plant-microbe-soil interactions as drivers of plant community structure and dynamics. Ecology 84:2281-2291.

Rillig, M. C., J. Antonovics, T. Caruso, A. Lehmann, J. R. Powell, S. D. Veresoglou, and E. Verbruggen. 2015. Interchange of entire communities: microbial community coalescence. Trends in Ecology \& Evolution 30:470-476. 
Rillig, M. C., A. Lehmann, C. A. Aguilar-Trigueros, J. Antonovics, T. Caruso, S. Hempel, J. Lehmann, K. Valyi, E. Verbruggen, S. D. Veresoglou, and J. R. Powell. 2016a. Soil microbes and community coalescence. Pedobiologia 59:37-40.

Rillig, M. C., A. Tsang, and J. Roy. 2016b. Microbial community coalescence for microbiome engineering. Frontiers in Microbiology 7:1967.

Rowe, H. I., C. S. Brown, and V. P. Claassen. 2007. Comparisons of mycorrhizal responsiveness with field soil and commercial inoculum for six native montane species and Bromus tectorum. Restoration Ecology 15:44-52.

Ruess, L., and P. M. Chamberlain. 2010. The fat that matters: soil food web analysis using fatty acids and their carbon stable isotope signature. Soil Biology and Biochemistry 42:1898-1910.

Santi, C., D. Bogusz, and C. Franche. 2013. Biological nitrogen fixation in non-legume plants. Annals of Botany 111:743-767.

Schadt, C. W., A. P. Martin, D. A. Lipson, and S. K. Schmidt. 2003. Seasonal dynamics of previously unknown fungal lineages in tundra soils. Science 301:1359-1361.

Schaminée, J. H. J., A. H. F. Stortelder, and E. J. Weeda. 1996. De vegetatie van Nederland. Deel 3. Plantengemeenschappen van graslanden, zomen en droge heiden. Opulus Press, Uppsala, Sweden.

Schaminée, J. H. J., E. J. Weeda, and V. Westhoff. 1998. De vegetatie van Nederland. Deel 4. Plantengemeenschappen van de kust en van binnenlandse pioniersmilieus. Opulus Press, Uppsala, Sweden.

Schelfhout, S., A. De Schrijver, S. De Bolle, L. De Gelder, A. Demey, T. Du Pré, S. De Neve, G. Haesaert, K. Verheyen, and J. Mertens. 2015. Phosphorus mining for ecological restoration on former agricultural land. Restoration Ecology 23:842-851.

Scherber, C., N. Eisenhauer, W. W. Weisser, B. Schmid, W. Voigt, M. Fischer, E.-D. Schulze, C. Roscher, A. Weigelt, E. Allan, H. Beßler, M. Bonkowski, N. Buchmann, F. Buscot, L. W. Clement, A. Ebeling, C. Engels, S. Halle, I. Kertscher, A.-M. Klein, R. Koller, S. König, E. Kowalski, V. Kummer, A. Kuu, M. Lange, D. Lauterbach, C. Middelhoff, V. D. Migunova, A. Milcu, R. Müller, S. Partsch, J. S. Petermann, C. Renker, T. Rottstock, A. Sabais, S. Scheu, J. Schumacher, V. M. Temperton, and T. Tscharntke. 2010. Bottom-up effects of plant diversity on multitrophic interactions in a biodiversity experiment. Nature 468:553-556.

Schnitzer, S. A., J. N. Klironomos, J. HilleRisLambers, L. L. Kinkel, P. B. Reich, K. Xiao, M. C. Rillig, B. A. Sikes, R. M. Callaway, S. A. Mangan, E. H. Van Nes, and M. Scheffer. 2011. Soil microbes drive the classic plant diversity-productivity pattern. Ecology 92:296-303.

Schrama, M., G. F. Veen, E. S. Bakker, J. L. Ruifrok, J. P. Bakker, and H. Olff. 2013. An integrated perspective to explain nitrogen mineralization in grazed ecosystems. Perspectives in Plant Ecology, Evolution and Systematics 15:32-44.

Shannon, S., S. L. Flory, and H. Reynolds. 2012. Competitive context alters plant-soil feedback in an experimental woodland community. Oecologia 169:235-243. 
Silvertown, J. 2004. Plant coexistence and the niche. Trends in Ecology \& Evolution 19:605-611.

Silvertown, J., P. Poulton, E. Johnston, G. Edwards, M. Heard, and P. M. Biss. 2006. The Park Grass Experiment 1856-2006: its contribution to ecology. Journal of Ecology 94:801-814.

Smith, S. E., and D. J. Read. 1997. Mycorrhizal symbiosis. Academic Press, San Diego, CA, USA.

Stopnisek, N., D. Zühlke, A. Carlier, A. Barberán, N. Fierer, D. Becher, K. Riedel, L. Eberl, and L. Weisskopf. 2015. Molecular mechanisms underlying the close association between soil Burkholderia and fungi. The ISME Journal 10:253-264.

Strong, D. R., H. K. Kaya, A. V. Whipple, A. L. Child, S. Kraig, M. Bondonno, K. Dyer, and J. L. Maron. 1996. Entomopathogenic nematodes: natural enemies of root-feeding caterpillars on bush lupine. Oecologia 108:167-173.

Suding, K. N., K. L. Gross, and G. R. Houseman. 2004. Alternative states and positive feedbacks in restoration ecology. Trends in Ecology \& Evolution 19:46-53.

Suleiman, A. K. A., L. Manoeli, J. T. Boldo, M. G. Pereira, and L. F. W. Roesch. 2013. Shifts in soil bacterial community after eight years of land-use change. Systematic and Applied Microbiology 36:137-144.

Svenning, J.-C., S. Normand, and F. Skov. 2008. Postglacial dispersal limitation of widespread forest plant species in nemoral Europe. Ecography 31:316-326.

Swift, M. J., O. W. Heal, and J. M. Anderson. 1979. Decomposition in terrestrial ecosystems. Blackwell Scientific Publications, Oxford, UK.

Tamme, R., I. Hiiesalu, L. Laanisto, R. Szava-Kovats, and M. Pärtel. 2010. Environmental heterogeneity, species diversity and co-existence at different spatial scales. Journal of Vegetation Science 21:796-801.

Tatineni, S., R. A. Graybosch, G. L. Hein, S. N. Wegulo, and R. French. 2010. Wheat cultivarspecific disease synergism and alteration of virus accumulation during co-infection with wheat streak mosaic virus and Triticum mosaic virus. Phytopathology 100:230-238.

Ter Steege, H., and R. Zagt. 2002. Density and diversity. Nature 417:698-699.

Teste, F. P., P. Kardol, B. L. Turner, D. A. Wardle, G. Zemunik, M. Renton, and E. Laliberté. 2017. Plant-soil feedback and the maintenance of diversity in Mediterranean-climate shrublands. Science 355:173-176.

Tilman, D. 1982. Resource competition and community structure. Princeton University Press, Princeton, NJ, USA.

Tilman, D. 1994. Competition and biodiversity in spatially structured habitats. Ecology 75:2-16.

Tilman, D. 2011. Diversification, biotic interchange, and the universal trade-off hypothesis. The American Naturalist 178:355-371.

Torsvik, V., L. Øvreås, and T. F. Thingstad. 2002. Prokaryotic diversity-magnitude, dynamics, and controlling factors. Science 296:1064-6. 
Troelstra, S. R., R. Wagenaar, W. Smant, and B. A. M. Peters. 2001. Interpretation of bioassays in the study of interactions between soil organisms and plants: involvement of nutrient factors. New Phytologist 150:697-706.

Tsiafouli, M. A., E. Thébault, S. P. Sgardelis, P. C. De Ruiter, W. H. Van der Putten, K. Birkhofer, L. Hemerik, F. T. De Vries, R. D. Bardgett, M. V. Brady, L. Bjornlund, H. B. Jørgensen, S. Christensen, T. D’Hertefeldt, S. Hotes, W. H. G. Hol, J. Frouz, M. Liiri, S. R. Mortimer, H. Setälä, J. Tzanopoulos, K. Uteseny, V. Pižl, J. Stary, V. Wolters, and K. Hedlund. 2015. Intensive agriculture reduces soil biodiversity across Europe. Global Change Biology 21:973-985.

Turnbull, L. A., M. J. Crawley, and M. Rees. 2000. Are plant populations seed-limited? A review of seed sowing experiments. Oikos 88:225-238.

Turrini, A., and M. Giovannetti. 2012. Arbuscular mycorrhizal fungi in national parks, nature reserves and protected areas worldwide: a strategic perspective for their in situ conservation. Mycorrhiza 22:81-97.

Van de Voorde, T. F. J., W. H. Van der Putten, and T. M. Bezemer. 2012. The importance of plantsoil interactions, soil nutrients, and plant life history traits for the temporal dynamics of Jacobaea vulgaris in a chronosequence of old-fields. Oikos 121:1251-1262.

Van de Voorde, T. F. J., W. H. Van der Putten, and T. M. Bezemer. 2011. Intra- and interspecific plant-soil interactions, soil legacies and priority effects during old-field succession. Journal of Ecology 99:945-953.

Van den Brink, P. J., and C. J. F. Ter Braak. 1999. Principal response curves: analysis of timedependent multivariate responses of biological community to stress. Environmental Toxicology and Chemistry 18:138-148.

Van der Bij, A. U., M. Pawlett, J. A. Harris, K. Ritz, and R. van Diggelen. 2016. Soil microbial community assembly precedes vegetation development after drastic techniques to mitigate effects of nitrogen deposition. Biological Conservation: in press (doi: 10.1016/j.biocon.2016.09.008).

Van der Heijden, M. G. A., R. D. Bardgett, and N. M. van Straalen. 2008. The unseen majority: soil microbes as drivers of plant diversity and productivity in terrestrial ecosystems. Ecology letters 11:296-310.

Van der Heijden, M. G. A., and M. Hartmann. 2016. Networking in the plant microbiome. PLOS Biol 14:e1002378.

Van der Maarel, E., and M. T. Sykes. 1993. Small-scale plant species turnover in a limestone grassland: the carousel model and some comments on the niche concept. Journal of Vegetation Science 4:179-188.

Van der Meijden, E., and R. E. Van der Waals-Kooi. 1979. The population ecology of Senecio jacobaea in a sand dune system: I. reproductive strategy and the biennial habit. Journal of Ecology 67:131-153.

Van der Meijden, R. 2005. Heukels' flora van Nederland. Wolters-Noordhoff, Groningen, the Netherlands. 
Van der Putten, W. H. 2003. Plant defence belowground and spatiotemporal processes. Ecology 84:2269-2280.

Van der Putten, W. H., R. D. Bardgett, J. D. Bever, T. M. Bezemer, B. B. Casper, T. Fukami, P. Kardol, J. N. Klironomos, A. Kulmatiski, J. A. Schweitzer, K. N. Suding, T. F. J. Van de Voorde, and D. A. Wardle. 2013. Plant-soil feedbacks: the past, the present and future challenges. Journal of Ecology 101:265-276.

Van der Putten, W. H., M. A. Bradford, E. P. Brinkman, T. F. J. Van de Voorde, and G. F. Veen. 2016. Where, when and how plant-soil feedback matters in a changing world. Functional Ecology 30:1109-1121.

Van der Putten, W. H., S. R. Mortimer, C. van Dijk, V. K. Bron, K. Hedlund, C. RodriguezBarrueco, J. Roy, T. A. Diaz Len, D. Gormsen, G. W. Korthals, S. Lavorel, I. Santa Regina, and P. Smilauer. 2000. Plant species diversity as a driver of early succession in abandoned fields : a multi-site approach. Oecologia 124:91-99.

Van der Putten, W. H., C. Van Dijk, and B. A. M. Peters. 1993. Plant-specific soil-borne diseases contribute to succession in foredune vegetation. Nature 362:53-56.

Van der Putten, W. H., C. Van Dijk, and S. R. Troelstra. 1988. Biotic soil factors affecting the growth and development of Ammophila arenaria. Oecologia 76:313-320.

Van der Wal, A., W. De Boer, I. M. Lubbers, and J. A. Van Veen. 2007. Concentration and vertical distribution of total soil phosphorus in relation to time of abandonment of arable fields. Nutrient Cycling in Agroecosystems 79:73-79.

Van der Wal, A., J. A. Van Veen, W. Smant, H. T. S. Boschker, J. Bloem, P. Kardol, W. H. Van der Putten, and W. De Boer. 2006. Fungal biomass development in a chronosequence of land abandonment. Soil Biology and Biochemistry 38:51-60.

Van Elsas, J. D., M. Chiurazzi, C. A. Mallon, D. Elhottovā, V. Krištůfek, and J. F. Salles. 2012. Microbial diversity determines the invasion of soil by a bacterial pathogen. Proceedings of the National Academy of Sciences 109:1159-1164.

Van Straalen, N. M., and P. C. Rijninks. 1982. Efficiency of Tullgren apparatus with respect to interpreting seasonal changes in age structure of soil arthropod populations. Pedobiologia 24:197-209.

Vécrin, M. P., and S. Muller. 2003. Top-soil translocation as a technique in the re-creation of speciesrich meadows. Applied Vegetation Science 6:271-278.

Veen, G. F., S. De Vries, E. S. Bakker, W. H. Van der Putten, and H. Olff. 2014. Grazing-induced changes in plant-soil feedback alter plant biomass allocation. Oikos 123:800-806.

Vellend, M. 2003. Habitat loss inhibits recovery of plant diversity as forests regrow. Ecology 84:1158-1164.

Veresoglou, S. D., J. M. Halley, and M. C. Rillig. 2015. Extinction risk of soil biota. Nature Communications 6:8862. 
Verhagen, R., J. Klooker, J. P. Bakker, and R. Van Diggelen. 2001. Restoration success of lowproduction plant communities on former agricultural soils after top-soil removal. Applied Vegetation Science 4:75-82.

Verschoor, B. C., R. G. M. de Goede, F. W. De Vries, and L. Brussaard. 2001. Changes in the composition of the plant-feeding nematode community in grasslands after cessation of fertiliser application. Applied Soil Ecology 17:1-17.

Viketoft, M., B. Sohlenius, S. Boström, C. Palmborg, J. Bengtsson, M. P. Berg, and K. HussDanell. 2011. Temporal dynamics of soil nematode communities in a grassland plant diversity experiment. Soil Biology and Biochemistry 43:1063-1070.

Vitousek, P. M., H. A. Mooney, J. Lubchenco, and J. M. Melillo. 1997. Human domination of Earth's ecosystems. Science 277:494-499.

Vivian-Smith, G. 1997. Microtopographic heterogeneity and floristic diversity in experimental wetland communities. Journal of Ecology 85:71-82.

Walker, K. J., P. A. Stevens, D. P. Stevens, J. O. Mountford, S. J. Manchester, and R. F. Pywell. 2004. The restoration and re-creation of species-rich lowland grassland on land formerly managed for intensive agriculture in the UK. Biological Conservation 119:1-18.

Walker, L. R., and R. del Moral. 2003. Primary succession and ecosystem rehabilitation. Cambridge University Press, Cambridge, UK.

Walker, L. R., J. Walker, and R. J. Hobbs. 2007. Linking restoration and ecological succession. Springer, New York, NY, USA.

Walker, L. R., and D. A. Wardle. 2014. Plant succession as an integrator of contrasting ecological time scales. Trends in Ecology \& Evolution 29:504-510.

Wallin, L., B. M. Svensson, and M. Lönn. 2009. Artificial dispersal as a restoration tool in meadows: sowing or planting? Restoration Ecology 17:270-279.

Wardle, D. A., R. D. Bardgett, J. N. Klironomos, H. Setälä, W. H. van der Putten, and D. H. Wall. 2004. Ecological linkages between aboveground and belowground biota. Science 304:1629-33.

Wardle, D. A., O. Zackrisson, G. Hörnberg, and C. Gallet. 1997. The influence of island area on ecosystem properties. Science 277:1296-1299.

Warren, J., A. Christal, and F. Wilson. 2002. Effects of sowing and management on vegetation succession during grassland habitat restoration. Agriculture, Ecosystems \& Environment 93:393-402.

White, T. J., T. D. Bruns, S. B. Lee, and J. W. Taylor. 1990. Analysis of phylogenetic relationships by amplification and direct sequencing of ribosomal DNA genes. Pages 315-322 in M. A. E. A. Innis, editor. PCR protocols. A guide to methods and applications. Academic Press, San Diego, CA, USA.

Whittaker, R. H. 1960. Vegetation of the Siskiyou Mountains, Oregon and California. Ecological Monographs 30:279-338. 
Wijesinghe, D. K., E. A. John, and M. J. Hutchings. 2005. Does pattern of soil resource heterogeneity determine plant community structure? An experimental investigation. Journal of Ecology 93:99-112.

Wilson, J. B. 2011. The twelve theories of co-existence in plant communities: the doubtful, the important and the unexplored. Journal of Vegetation Science 22:184-195.

Wilson, J. B., R. K. Peet, J. Dengler, and M. Pärtel. 2012. Plant species richness: the world records. Journal of Vegetation Science 23:796-802.

Wilson, J. B., and S. H. Roxburgh. 1994. A demonstration of guild-based assembly rules for a plant community, and determination of intrinsic guilds. Oikos 69:267-276.

Wood, S. 2006. Generalized additive models: an introduction with R. CRC Press, Boca Raton, FL, USA.

Yeates, G. W. 2003. Nematodes as soil indicators: functional and biodiversity aspects. Biology and Fertility of Soils 37:199-210.

Yeates, G. W., T. Bongers, R. G. M. De Goede, D. W. Freckman, and S. S. Georgieva. 1993. Feeding habits in soil nematode families and genera—an outline for soil ecologists. Journal of Nematology 25:315-331.

Young, T. P., J. M. Chase, and R. T. Huddleston. 2001. Community succession and assembly: comparing, contrasting and combining paradigms in the context of ecological restoration. Ecological Restoration 19:5-18.

Young, T. P., D. A. Petersen, and J. J. Clary. 2005. The ecology of restoration: historical links, emerging issues and unexplored realms. Ecology Letters 8:662-673.

Zar, J. H. 1999. Biostatistical analysis. 4th edition. Prentice Hall, Upper Saddle River, NJ, USA.

Zhang, N., W. H. Van der Putten, and G. F. Veen. 2016. Effects of root decomposition on plant-soil feedback of early- and mid-successional plant species. New Phytologist 212:220-231.

Zimdars, B., and W. Dunger. 1994. Synopses on palaearctic collembola, volume 1: Tullbergiinae. State Museum of the Natural History Museum of Gorlitz, Görlitz.

Zobel, M. 1997. The relative of species pools in determining plant species richness: an alternative explanation of species coexistence? Trends in Ecology \& Evolution 12:266-269.

Zobel, M., M. Otsus, J. Liira, M. Moora, and T. Möls. 2000. Is small-scale species richness limited by seed availability or microsite availability? Ecology 81:3274-3282.

Zuur, A. F., E. N. Ieno, and C. S. Elphick. 2010. A protocol for data exploration to avoid common statistical problems. Methods in Ecology and Evolution 1:3-14.

Zuur, A. F., E. N. Ieno, N. J. Walker, A. A. Saveliev, and G. M. Smith. 2009. Mixed effects models and extensions in ecology with R. Springer, New York, NY, USA. 


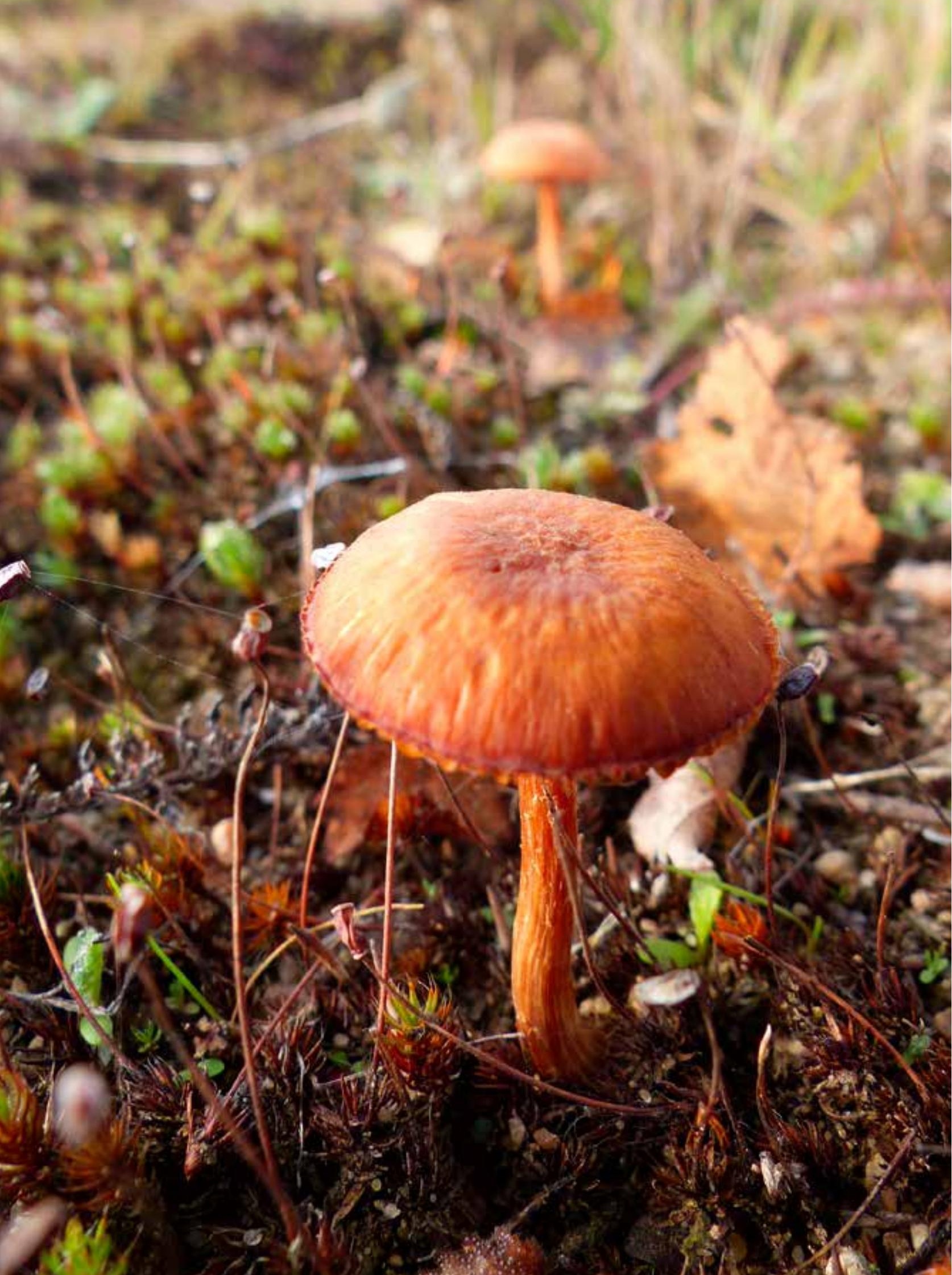


Summary / samenvatting 
Biodiversity is declining worldwide and many ecosystems have been degraded due to human actions. There have been many attempts to restore degraded ecosystems, but restoration success varies. Past human management has left important abiotic and biotic legacies and active intervention is needed to overcome these legacies. Legacy effects include altered abiotic conditions and limited availability of appropriate seeds. However, plants also have many interactions with the myriad organisms that inhabit the soil. Soil biota include e.g. bacteria, fungi, nematodes, collembolan, and mites. Restoring plant-soil interactions may be key to successful ecological restoration, because studies on natural succession in ecosystems show that both plant and soil communities develop in concert. In addition, latesuccessional soil communities promote the performance of late-succession plant species that are often the target species for restoration. The aims of my thesis were to 1) test whether inoculation of living soil can improve restoration of speciesrich grasslands and dry heathlands, and 2) understand how plant-soil interactions affect plant composition and diversity.

In a large-scale field experiment, called "Reijerscamp-experiment", I tested the potential of soil inoculation to speed up ecosystem restoration. On a former arable field large areas of on average 0.5 ha were inoculated with a thin layer of $<1 \mathrm{~cm}$ living soil, which was taken either from a mid-succession grassland or a dryheathland. After six years I monitored the species composition of the vegetation and the soil community. I found that both types of inoculum had substantially altered the community composition of both soil and vegetation. Moreover, the soil inocula had caused a shift in the direction of the respective donor communities. In a parallel mesocosm experiment I repeated the experiment while sowing a standardized species-rich seed mixture to ensure that seed availability was the same in all treatments. Also in this case the sown plant community developed towards the respective communities found in the donor sites. Consequently the soil community is, at least in part, able to steer plant community composition in the field.

I also tested how mixtures of inocula from different donor systems affect restoration success. In a greenhouse experiment I made replacement series of soil inocula sourced from arable fields, mid-succession grasslands and dry heathlands and monitored the responses of target and ruderal plant species. The target species all responded positively to higher proportions of heathland material in the inoculum, while the responses of the ruderal species were variable. Interestingly, a 50:50 mixture of arable and heathland inoculum strongly reduced the growth of the ruderal species. Soil inoculation may be considered as a way of microbiome 
engineering, which is a newly emerging field mainly used to improve human health and agricultural production. My results show that conceptually similar techniques can be applied to improve inocula for the restoration of ecological communities.

In a second field experiment I tested the long-term consequences of soil inoculation with and without sowing mid-successional plant species for plant and soil community composition. I found that sowing strongly altered plant community composition for over two decades. Soil inoculation, on the other hand, substantially altered the composition of the soil nematode community and that these effects persisted for at least 15 years. This shows that both sowing and soil inocualtion can have long-term impacts on community development. However, in contrast to the Reijerscamp experiment, the effect of soil inoculation on vegetation composition was transient. I propose that in this case the presence of an intact arable topsoil, as well as perhaps a too minimal difference between the composition of the donor and recipient soil communities may have limited the impact of the soil inocula.

In general, the restoration of plant cover and a number of common ('matrix') plant species can be achieved using standard approaches, e.g. reducing site fertility and providing seed material, but creating conditions that allow for coexistence of both locally dominant and rare subordinate species proves much more elusive. Fundamental knowledge on how biodiversity is regulated is needed to restore diverse plant communities including the rare species. Testing plant-soil feedback provides a way to directly study the net consequences of the myriad interactions between plants and soil biota for plant performance and community composition. However, while both plants and soil communities are strongly heterogeneous in space and time, spatiotemporally explicit tests of plant-soil feedback are still rare.

In a greenhouse experiment I studied how spatial heterogeneity in plant-soil feedbacks influence plant communities. I found that when multiple species conditioned the soil, plant performance was reduced compared to monospecific soil conditioning. This reduction in competitive ability led to a higher plant diversity in the experimental communities. The plant responses were not related to differences in abiotic conditions, but soil conditioning induced clear changes in fungal community composition. Recent meta-analyses and experiments have shown that spatial heterogeneity in abiotic conditions only promotes plant diversity when the grain of the heterogeneity is larger than the size of individual plants. When it is smaller, heterogeneity selects for those species that have the highest root plasticity and this leads to lower plant diversity. Together, these results suggest 
that spatial heterogeneity in abiotic conditions promotes plant beta diversity, while interaction with the soil community, primarily soil-borne antagonists, maintains plant alpha diversity.

Finally, I used repeated soil conditioning by conspecific and heterospecific species to show that soil feedbacks may carry over across soil conditioning periods. In contrast to what is commonly assumed my data show that heterospecific soil-conditioning can result in equally negative plant-soil feedback as repeated conspecific soilconditioning and repeated conspecific soil-conditioning does not always lead to stronger negative feedback. Instead, the particular sequence of plant species that successively condition the soil strongly determines the sign and magnitude of plant-soil feedback. These results highlight the need to incorporate sequential soilconditioning in models of plant communities and effective crop-rotations.

In conclusion, plant-soil interactions are a key aspect in the natural dynamics of plant communities and can be used to improve restoration of semi-natural ecosystems. Abiotic conditions and dispersal ability determine which species may occur in a given site. However, at small spatial scales plant-soil feedbacks and particularly interactions with soil borne antagonists can enhance plant species diversity. Manipulation of the soil community, through inoculation of soil from well-developed donor sites can speed up natural succession and even steer its direction in the field. However, soil inoculation success will not be universal and depends on the match in abiotic conditions of donor and recipient sites, as well as the community composition of the inoculum and the resident communities. Future studies are needed to test the success of introducing soil communities across environmental gradients. 


\section{Samenvatting}

Wereldwijd vermindert de biodiversiteit en veel ecosystemen zijn gedegradeerd door menselijk handelen. Het is vaak geprobeerd om gedegradeerde ecosystemen te herstellen, maar de mate van succes varieert. Het voormalig beheer heeft tot sterke abiotische en biotische nalatenschap geleid en actief ingrijpen is nodig om dit nalatenschap te doorbreken. Deze 'nalatenschaps'-effecten omvatten veranderde abiotische omstandigheden en beperkte beschikbaarheid van geschikte zaden. Daarnaast gaan planten ook meerdere interacties aan met een veelvoud aan organismen die in de bodem leven. Het bodemleven omvat o.a. bacteriën, schimmels, nematoden (ook wel aaltjes genoemd), springstaarten en mijten. Het herstel van deze plant-bodem interacties speelt mogelijk een sleutelrol in ecologisch gebiedsherstel. Uit studies naar natuurlijke successie in ecosystemen blijkt namelijk dat zowel de planten als de bodemgemeenschap zich in een samenspel ontwikkelen. Daarnaast bleek dat bodemgemeenschappen uit laat-successie systemen de groei van planten uit die systemen bevorderen, en deze soorten zijn vaak de doelsoorten voor de natuurbescherming. Mijn proefschrift had een tweeledig doel: 1) het testen of inoculatie met levende bodem het ecologisch herstel van soortenrijke graslanden en droge heiden kan verbeteren en 2) begrijpen hoe plant-bodem interacties de samenstelling en diversiteit van plantengemeenschappen beïnvloeden.

In een grootschalig veldexperiment, het "Reijerscamp-experiment" genoemd, heb ik de potentie van bodem-inocula voor het versnellen van ecosysteemherstel getest. In een voormalige akker werden grote stukken van gemiddelde 0.5 hectare geïnoculeerd met een dunne laag van $<1 \mathrm{~cm}$ levende bodem. Deze inocula werden verzameld uit een mid-successie grasland of een droog heidegebied. Na zes jaar heb ik de soortensamenstelling van de vegetatie en de bodemgemeenschap in kaart gebracht. Ik ontdekte dat beide typen inocula de samenstelling van de vegetatie en de bodemgemeenschap substantieel hadden veranderd. Bovendien veroorzaakten de inocula een verschuiving in de richting van de twee respectievelijke donorgemeenschappen. In een parallel mesocosmos experiment heb ik het experiment herhaald waarbij een gestandaardiseerd soortenrijk zadenmengsel werd gezaaid zodat het zadenaanbod in alle behandelingen gelijk was. Ook hier bleek dat de gezaaide plantengemeenschap zich ontwikkelde in de richting van de donorgemeenschappen. Hieruit blijkt dat de bodemgemeenschap, ten minste deels, in staat is om de plantengemeenschap in het veld te sturen.

Verder heb ik onderzocht hoe mengsels van inocula uit verschillende donorecosystemen het ecologisch herstel beïnvloedden. In een kasproef heb ik bodem- 
inocula met verschillende verhoudingen bodem uit akkers, mid-successie graslanden en droge heiden getest op de groei van ruderale- en doelsoorten. De doelsoorten groeiden beter met een hoger aandeel heidegrond in het inoculum, terwijl de onkruiden variabel reageerden op dit inoculum. Interessant is dat een 50:50 mengel van akker- en heidebodem de groei van ruderale soorten sterk remde. Bodem-inoculatie kan gezien worden als een vorm van microbioom beheer, dit is een nieuw onderzoeksveld - voornamelijk gericht op het verbeteren van de menselijke gezondheid en agrarische productie. Mijn resultaten laten zien dat conceptueel vergelijkbare technieken toegepast kunnen worden om bodeminocula ten behoeve van ecologisch gebiedsherstel te verbeteren.

In een tweede veldproef heb ik de langetermijn-consequenties van bodem-inoculatie getest voor de soortensamenstelling van de planten- en bodemgemeenschap, zowel met en zonder het zaaien van zaden van mid-successie plantensoorten. Hieruit bleek dat zaaien de samenstelling van de vegetatie sterk beïnvloedde gedurende ten minste twee deccenia. Bodem-inoculatie daarentegen beinvloedde de samenstelling van de nematoden gemeenschap in de bodem en deze effecten waren meetbaar gedurende ten minste vijftien jaar. Dit laat zien dat beide ingrepen over de lange termijn doorwerken in de ontwikkeling van de lokale gemeenschap. In tegenstelling tot het Reijerscamp-experiment was de invloed van het bodem-inoculum op de vegetatie van korte duur. Mogelijk hebben in dit geval de aanwezigheid van een intacte akkerbodem toplaag (i.e. niet ontgrond) en het vermoedelijk kleine verschil in soortensamenstelling in de donor en de ontvangende bodemgemeenschap de impact van de bodem-inocula beperkt.

Over het algemeen is het mogelijk om de plantenbedekking en de aanwezigheid van een aantal algemene ('matrix') plantensoorten te herstellen door middel van standaardingrepen, bijvoorbeeld het verminderen van nutriëntgehaltes in de bodem en het aanbieden van zaden. Echter het scheppen van de condities die nodig zijn voor het samenleven van zowel lokaal dominante als zeldzame ondergeschikte soorten blijft vooralsnog ongrijpbaar. Fundamentele kennis over hoe biodiversiteit wordt gereguleerd is nodig om diverse plantengemeenschappen, inclusief zeldzame soorten, te kunnen herstellen. Proeven met betrekking tot de wisselwerking tussen planten en bodems (vertaling plant-soil feedback) maken het mogelijk om direct de netto gevolgen van de veelvoud aan mogelijke interacties tussen planten en bodemorganismen voor het functioneren en de samenstelling van de plantengemeenschap te bestuderen. Alhoewel zowel planten- als bodemgemeenschappen zeer heterogeen zijn in ruimte ene tijd, zijn studies naar 
de plant-bodem wisselwerking nog maar zelden gedaan in ruimtelijk en temporeel expliciete experimenten.

Daarom bestudeerde ik in een kasproef hoe ruimtelijk heterogeniteit in plantbodemwisselwerking van invloed is op plantengemeenschappen. Ik ontdekte dat als meerdere soorten de bodem geconditioneerd hadden dat de groei van planten lager was dan in bodem die door een enkele soort was geconditioneerd. Deze vermindering in competitief vermogen veroorzaakte een hogere plantendiversiteit in de experimentele gemeenschappen. De gemeten responsen van de planten konden niet gerelateerd worden aan verschillen in abiotische omstandigheden, maar bodem-conditionering leidde tot duidelijke veranderingen in de soortensamenstelling van de schimmelgemeenchap in de bodem. Recente metaanalyses en experimenten hebben laten zien dat ruimtelijke heterogeniteit in abiotisch omstandigheden alleen de plantendiversiteit bevorderen als schaal van de heterogeniteit groter is dan de grootte van de individuele planten. Als de bodem heterogeen is op een kleinere schaal dan leidt dit slechts tot selectie voor die soorten die een plastisch wortelstelsel bezitten en dit heeft een lagere plantendiversiteit tot gevolg. Gezamenlijk doen deze resultaten vermoeden dat ruimtelijke heterogeniteit in abiotische omstandigheden voor planten de beta-diversiteit bevorderd, terwijl de interacties met de bodemgemeenschap, en primair de bodemgebonden ziekten en plagen, de alfa-diversiteit op peil houdt.

Tot slot heb ik door middel van herhaalde bodem-conditionering met dezelfde en andere soorten kunnen laten zien dat de nalatenschap van de plant-bodem wisselwerking over verschillende conditioneringsperioden kan doorwerken. In tegenstelling tot wat algemeen wordt aangenomen laten mijn data zien dat bodemconditionering door andere soorten een even negatieve plant-bodem wisselwerking tot gevolg kan hebben als herhaalde conditionering met dezelfde soort. Daarnaast wordt de negatieve wisselwerking door herhaalde conditionering met dezelde soort niet noodzakelijkerwijs versterkt. In plaats daarvan blijkt dat de specifieke sequentie van bodem-conditionerende plantensoorten in sterke mate bepalend is voor de mate waarin de plant-bodem wisselwerking versterkend dan wel afremmend werkt. Deze resultaten onderstrepen de noodzaak om herhaalde bodem-conditionering op te nemen in modellen van plantengemeenschappen en vruchtwisselingsschema’s.

Concluderend, plant-bodem interacties spelen een sleutelrol in de natuurlijke dynamiek van plantengemeenschappen en kunnen gebruikt worden om het herstel van half-natuurlijke ecosystemen te bevorden. Abiotisch omstandigheden en het 
vermogen tot verspreiding bepalen welke soorten in een gebied kunnen voorkomen, maar op kleine ruimtelijke schalen bevorderen plant-bodem wisselwerking en in het bijzonder de interactie met bodemgebonden antagonisten de plantendiversiteit. Het manipuleren van de bodemgemeenschap door middel van het inoculeren van bodem uit goed ontwikkelde donorgebieden kan de natuurlijke successie versnellen en op deze manier kan zelfs de richting van successie in het veld gestuurd worden. Het succes van bodem-inoculatie is niet universeel en hangt af van de overeenkomst in abiotische omstandigheden van het ontvangende en het donorgebied, als ook van de soortensamenstelling van het inoculum en de ontvangende gemeenschap. Meer onderzoek is nodig om het succes van bodeminoculatie over brede gradiënten in omgevingsfactoren te testen. 


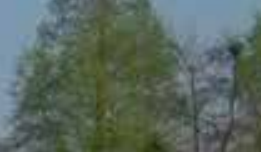
1

iI
ntwiso

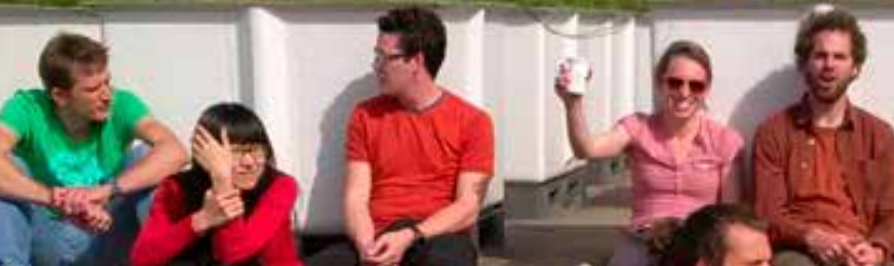

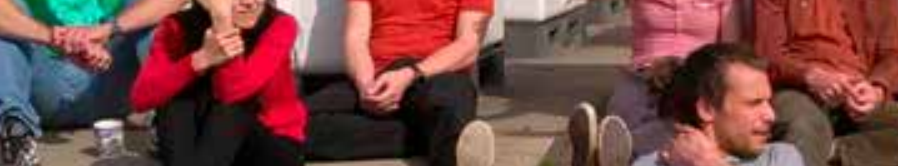
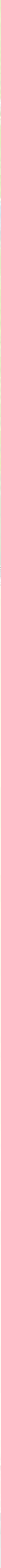
Acknowledgements / dankwoord 
Bedankt Martijn en Wim voor jullie begeleiding van mijn promotietraject en voor het vertrouwen dat jullie toen en ook nu weer in mij gesteld hebben. Jullie hebben mij nieuwe perspectieven op het wetenschappelijk bedrijf gegeven. Martijn, jouw scherpe en, ik mag wel zeggen, hyper-creatieve brein zijn voor mij een grote inspiratie. Een manuscript wordt door jou met wervelwindsnelheid binnenstebuiten gekeerd en tot op het bot blootgelegd. Daarna wordt het in een nieuw kader geplaatst en leidt dit tot inzichten die ik niet had voorzien. We zijn het niet altijd eens geweest, maar desalniettemin heb ik erg veel van je kunnen leren! Dank voor alle hulp, de vrijheid die ik heb gekregen en je begrip en je betrokkenheid toen mijn gezondheid te wensen over liet. Wim, jouw vermogen om potentie te zien, in mensen en ideeën, is een uiterst waardevolle vaardigheid (of talent?) voor de wetenschap in het algemeen en de vorderingen van promovendi in het bijzonder. Je diplomatieke opstelling tijdens meetings (b.v. tijdens de 'overgrenzen'-workshop) heeft me veel bijgebracht over op constructieve wijze tot oplossingen komen. Dank voor jullie begeleiding - in samenspan heeft dit m.i. tot een aantal mooie stukken geleid en ik hoop dat dit de opmaat is naar een wetenschappelijk meesterschap in de toekomst.

Ciska (V.), voor een groot deel van mijn promotie zijn we partners in crime geweest - ook al had dit eigenlijk niets met dit proefschrift te maken! Eerst in het kader van het voedselbos dat we bij het NIOO opzetten, daarna in het kader van het 'over grenzen'-project. Veel dank voor al je energie, je optimisme en je nuchtere blik. Louise, dank voor het uitdragen van de ecologisch duurzaamheids-boodschap, je motiverende woorden, en je vermogen om mensen tot handelen te bewegen.

Machiel jij zag in het begin van deze eeuw de relevantie van een grootschalige veldproef met bodem-inoculaties - dit hebben jij en je team toen gerealiseerd. Dat experiment is het praktisch fundament onder mijn promotieonderzoek geworden. Dank voor je vertrouwen in de ecologische wetenschap, je ondersteuning van onze veldstudies en je blik vanuit de natuurbeheerpraktijk. Dank ook aan Lieuwe Haanstra, Henk Hofman, Herman Veerbeek, Leon Veiken, Annelies Blankena, en Ivo Roessink die gegevens verstrekten en verzamelden in en om de Reijerscamp of toegang verschaften tot terreinen.

Thanks to my students Quirine, Yiman, Glenn, Pauline, Tom, Hui, and Adina for the tremendous amount of work you have pulled off during your respective projects. Dank ook aan de analisten, assistenten en helpers in het veld: Roel, Ciska (R.), Iris, Gregor, Henk (D.), Freddy, Henk (M.), Slavica, Tanja, Erik, Jeroen, 
Gilles, Rebecca, Gerdien, Stijn (S.), Henk-Jan, Luit en Ingrid. Eke, bedankt voor je toewijding en altijd creatieve oplossingen. Florentine, Tess, Nina en Els, bedankt voor het organiseren van een groot deel van de niet-wetenschappelijke verplichtingen uit mijn projecten. Carel, Froukje, Monique, Perro en Brechtje, bedankt voor de ondersteuning bij het opstellen van persberichten en het maken van websites en video's. Carel veel dank voor de vele gesprekken die we hadden over het wetenschappelijk bedrijf, de samenleving en persoonlijke ontwikkeling. Zonder al jullie hulp was dit onderzoek zeker niet mogelijk geweest!

Many thanks to my colleagues in and outside NIOO, particularly Roeland, Maarten, Gera, Tom, Arjen (B.), Koen, Kelly, Wietse, Elizabeth Borer, John Moore, Richard Bardgett, Johan Six for many useful ideas and discussions. Maarten, jij weergaloze, energieke bolleboos - dank voor alle avonturen en gesprekken in de kroeg, de trein, Yorkshire Dales, Haarzuilens, en natuur-overig. Dank in het bijzonder aan Martine en Stijn voor de mogelijkheid om professionele en persoonlijke frustraties bij jullie te mogen spuien. Arjen (de G), jij stond (mede) aan de wieg van mijn onderzoekcarrière, dank voor alle toffe projecten en ideetjes die we samen bedacht en uitgevoerd hebben - op naar meer! Stefan und Kristin danke für den schönsten publications-erfahrung denn ich bis jetzt hatte! Lass uns schnell noch etwas schreiben. Sven, Jan and Ana, many thanks for our car travels from and to Wageningen. Thanks to all my roomies at NIOO through the years for creating a pleasant and productive work atmosphere: Kadri, Kelly, Tom, Stefan, Basten (Oeh! Dat ziet er interessant uit!), Roeland, Jingjing, Martine (Oké, één dag). Thanks to all $(\mathrm{PhD})$ colleagues at NIOO for making our institute such a great place to work - you're the most international, active and enthusiastic bunch I've met in science so far.

Dank aan de medewerkers van het PE\&RC-office, in het bijzonder Claudius en Lennart - jullie maken de PE\&RC tot de beste onderzoekschool in Nederland en waarschijnlijk het universum. Thanks also to all members of the PE\&RC PhD Council, in particular Mark and Chantal, for all the discussions and insight into the inner workings of academia beyond hands-on-research. Bijzonder veel dank aan Tryntsje, Nadia en Chantal voor het organiseren van de PhD speeddating event!

Lieve vrienden, heel veel dank dat jullie het met mij uithouden - sommigen al bijna 20 jaar! Voor alle etentjes, fantastische reizen, feestjes, bemoedigende woorden, kritische beschouwingen en nog zo veel meer. Kelly, dank dat je je als een tijger vastbijt en dat je mij op het juiste moment een zeer verstandige carrière-technische 
keuze hebt helpen maken. Inge bedankt voor de prachtige omslag om dit boekje. Leon, Luit, Kelly, Arianne, Yoran en Inge, dank dat jullie er altijd zijn geweest, door dik en dun.

Lieve familie dank voor de hechte clan die we samen vormen. Wim en Ieneke, dank voor jullie warme opvoeding, vol humor, reflectie, doorzettingsvermogen, sterke principes, zingeving, hoop, vergeving en waardering voor de natuur. Jullie zijn fantastische ouders. Jelmer dank dat je mijn toffe broer bent, voor je nuchtere kijk en je droge humor - hopelijk doe je op een gezette tijd ook een Brexit en strijk je ergens in de buurt neer. Johan en Altine, Tobias, Bernike, bedankt voor de manier waarop jullie mij in jullie clan hebben opgenomen.

Ruth, tot slot, dank voor al je liefde, vrolijkheid, openheid en warmte. Beter had ik niet kunnen dromen.

En voor iedereen die ik vergeten ben, enorm sorry, maar bedankt!

Utrecht, 22 April 2017 


\section{About the author}

Jasper Wubs was born in Kampen, the Netherlands, on February $8^{\text {th }}$, 1985 . He grew up in Steenwijk and went to high school in Meppel. There his teacher Henk van Netten got him interested in the science of biology - through direct engagement with experimentation. After a gap year working in a handicraft workshop with mentally-challenged individuals in Hungary, he started his biology

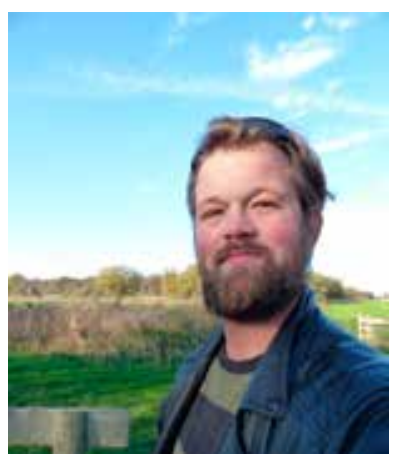
studies at Utrecht University and specialized in plant ecology. For his MSc-degree he researched the mating systems and colonization processes of ferns with dr. Heinjo During, dr. Roy Erkens and dr. Arjen de Groot. His second thesis was on the effects of snow-lie differences on summer carbon cycling in montane moss heath ecosystems in Scotland with dr. René van der Wal and dr. Sarah Woodin (both University of Aberdeen). After graduating (cum laude) he taught biostatistics and ecology as a teaching assistant and was a junior researcher in the Ecology and Biodiversity group at Utrecht University. In March 2012, he started his PhD-research on plant-soil interactions with a special focus on plant-soil feedback and nature restoration supervised by prof. Martijn Bezemer and prof. Wim van der Putten at the Netherlands Institute of Ecology (NIOO-KNAW), which led to this thesis. For his research and public outreach he received the 'Zilveren Parnassia' in 2016 - an award given annually to a socially engaged young scientist in the field of natural science, nature conservation and nature education. 


\section{List of publications}

\section{Peer-reviewed publications}

Schulz-Bohm, K, Geisen S, Wubs ERJ, Song C, De Boer W, Garbeva PV. 2017. The prey's scent - volatile-mediated interactions between soil bacteria and their protist predators. The ISME Journal 11: 817:820.

Wubs ERJ, Van der Putten WH, Bosch M, Bezemer TM. 2016. Soil inoculation steers restoration of terrestrial ecosystems. Nature Plants 2: 16107 - highlighted in Science 353: 203.

Wubs ERJ, Bezemer TM. 2016. Effects of spatial plant-soil feedback heterogeneity on plant performance in monocultures. Journal of Ecology 104: 364-376.

Wubs ERI, Fraaije RGA, Kleyheeg E, De Groot GA, Garssen AG, Erkens RHJ, Raven BM, Soons MB. 2016. Going against the flow: a case for upstream dispersal and detection of uncommon dispersal events. Freshwater Biology 61: 580-595.

Wipf S, Sommerkorn M, Stutter MI, Wubs ERJ, Van der Wal R. 2015. Snow cover, freeze-thaw, and the retention of nutrients in an oceanic mountain ecosystem. EcoSphere: 6(10): art207.

Robroek BJM, Wubs ERJ, Marti, M, Zając K, Andersen JP, Andersen A, Börjesson G, Bragazza L, Dise NB, Keuskamp JA, Larsson M, Lindgren P-E, Mattiasson P, Solomonsson J, Sundberg C, Svensson BH, Verhoeven JTA. 2014. Microclimatological consequences for plant and microbial composition in Sphagnum-dominated peatlands. Boreal Environment Research 19: 195-208.

De Groot GA, During HJ, Ansell SW, Schneider H, Bremer P, Wubs ERI, Maas JW, Korpelainen H, Erkens RHJ. 2012. Diverse spore rains and limited local exchange shaped fern genetic diversity in a recently created habitat colonized by long-distance dispersal. Annals of Botany: 109: 965-978.

De Groot GA, Verduyn B, Wubs ERJ, Erkens RHJ, During HJ. 2012. Inter- and intraspecific variation in fern mating systems after long-distance colonization: the importance of selfing. BMC Plant Biology 12: 3 . 
De Groot GA, Korpelainen H, Wubs ERJ, Erkens RHJ. 2011. Isolation of polymorphic microsatellite markers and tests of cross-amplification in four widespread European calcicole ferns. American Journal of Botany: 98: e319-e322.

Wubs ERJ, De Groot GA, During HJ, Vogel JC, Grundmann M, Bremer P, Schneider H. 2010 Mixed mating system in the fern Asplenium scolopendrium: implications for colonization potential. Annals of Botany 106: 583-590.

\section{Professional publications}

Wubs ERJ, Van der Putten, WH, Bosch M, Bezemer TM. 2016. Natuurherstel door grondtransplantatie? Landschap 33: 11-14.

Van Noppen F, Bosch M, Wubs ERJ, Haanstra L, Verbaan W, Van Houwelingen D, Philippona J, Van Ekeris R, Van der Putten WH, Bezemer TM. 2015. Afgraven, bodemtransplantaties en uitstrooien van maaisel op voormalige landbouwgronden: het Reijerscamp experiment. De Levende Natuur 116: 222-227. 
PE\&RC Training and Education Statement

With the training and education activities listed below the PhD candidate has complied with the requirements set by the C.T. de Wit Graduate School for Production Ecology and Resource Conservation (PE\&RC) which comprises of a minimum total of 32 ECTS (= 22 weeks of activities)

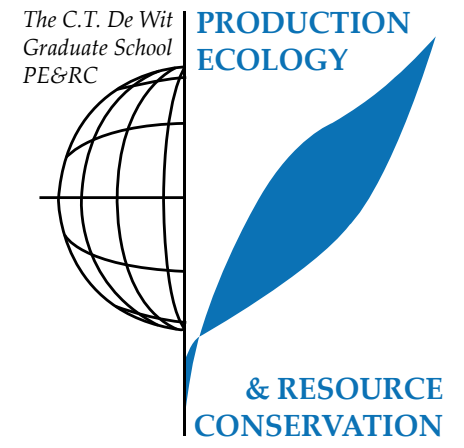

\section{Review of literature (6 ECTS)}

Spatial heterogeneity and plant diversity: changing relative contributions of nutrients and soil biota as a function of the grain of heterogeneity (2014)

\section{Post-graduate courses (9.5 ECTS)}

- International nematode identification course; Lab. of Nematology (2012)

- Bayesian statistics; Biometris (2012)

- Soil, biodiversity and life; PE\&RC (2012)

- Multivariate analysis of ecological data; Fact. Science, University of South Bohemia, Czech Republic (2013)

- Sampling in space and time for survey and monitoring of natural resources; PE\&RC (2013)

Invited review of (unpublished) journal manuscript (2 ECTS)

- Applied Vegetation Science: restoration of species rich grasslands (2013)

- Journal of Ecology: biotic and abiotic controls on succession (2015)

\section{Competence strengthening / skills courses (3.7 ECTS)}

- Project \& time management; WGS (2012)

- Teaching \& supervising MSc theses; Docentondersteuning (2013)

- Reviewing a scientific paper; WGS (2013)

- PhD Competence assessment; WGS (2014)

- Entrepreneurship in and outside science; WGS (2015)

PE\&RC Annual meetings, seminars and the PE\&RC weekend (2.1 ECTS)

- PE\&RC Weekend first year (2012)

- PE\&RC Day $(2014,2015)$ 
- PE\&RC Weekend last year (2015)

Discussion groups / local seminars / other scientific meetings (12 ECTS)

- TE-journal club (2012-2016)

- Modelling \& statistics network (2012-2014)

- Ecological theory \& application (2012-2016)

International symposia, workshops and conferences (13.5 ECTS)

- $56^{\text {th }}$ Annual Symposium of the International Association for Vegetation Science (IAVS) ; oral presentation; Tartu, Estonia (2013)

- $1^{\text {st }}$ Global Soil Biodiversity Initiative (GSBI) conference; poster presentation;

Dijon, France (2014)

- Centennial Meeting of the Ecological Society of America (ESA); oral presentation; Baltimore, MD, United States (2015)

- Annual Meeting British Ecological Society (BES); oral presentation; Edinburgh, Scotland, UK (2015)

- $46^{\text {th }}$ Annual Meeting of the Ecological Society of Germany, Austria and Switzerland; Gesellschaft fur Ökologie (GfÖ); oral presentation; Marburg, Germany (2016)

- Annual Meeting British Ecological Society (BES); oral presentation; Liverpool, UK (2016)

\section{Supervision of a MSc students (6 ECTS)}

- Effect of soil transplantation on biogeochemical cycling (Q. Hakkaart)

- Role of soil community composition in effect of soil inoculations (P. Melchers) 


\section{Colophon}

The research described in this thesis was conducted at the Department of Terrestrial Ecology of the Netherlands Institute of Ecology (NIOO-KNAW).

The research described in this thesis was financially supported by the Netherlands Organization for Scientific Research (NWO) as part of the 'Biodiversiteit werkt' programme (No. 841.11.008 to T.M. Bezemer).

This is NIOO thesis 143

Text \& figures: @ E.R.J. Wubs (e.r.j.wubs@gmail.com)

Cover design: Studio Miedema (www.studiomiedema.com)

Layout: E.R.J. Wubs

Photos: E.R.J. Wubs, F. ten Hooven, G. Hol

Printed by: Digiforce | Proefschriftmaken.nl 


\section{Propositions}

1. Soil inoculation is a powerful method to restore terrestrial ecosystems, but its success depends on the composition of both the donor and recipient soil.

(this thesis)

2. At the spatial scale where plants interact, plant diversity is maintained by pest pressure, rather than by competition for abiotic niches. (this thesis)

3. The fact that some ecological phenomena can be described by stochastic processes does not mean that chance is the underlying mechanism.

4. Surveys of standing genetic variation do not provide quantitative evidence of dispersal among plant populations.

5. Consuming meat at a conference on climate change is illogical if attendance is out of genuine concern for the negative consequences of climate change for mankind.

6. Success is a left-tailed, hump-shaped function of ambition.

Propositions belonging to the thesis, entitled:

The steering role of plant-soil interactions in natural community dynamics and nature restoration.

Engel Reinder Jasper Wubs

Wageningen, 31 May 2017 